\title{
CHARGE syndrome: candidate genes and pathogenesis
}

\author{
Dissertation
}

\author{
for the award of the degree \\ "Doctor rerum naturalium" \\ of the Georg-August-Universität Göttingen \\ within the doctoral program Genes and Development \\ of the Georg-August University School of Science (GAUSS)
}

\author{
submitted by \\ Yvonne Schulz \\ from Hamburg, Germany
}

Göttingen 2014 


\section{Thesis Committee}

Prof. Dr. med. Dr. h.c. Wolfgang Engel

Department of Human Genetics, Georg-August-University, Göttingen

Prof. Dr. Andreas Wodarz

Department of Microscopical Anatomy and Molecular Cell Biology, University Köln Medical Center, Köln

Prof. Dr. Ahmed Mansouri

Department of Molecular Cell Biology/Molecular Cell Differentiation, Max Planck Institute for biophysical Chemistry, Göttingen,

\section{Members of the Examination Board}

Referee: Prof. Dr. med. Dr. h.c. Wolfgang Engel

Department of Human Genetics, Georg-August-University, Göttingen

$2^{\text {nd }}$ Referee: Prof. Dr. Andreas Wodarz

Department of Microscopical Anatomy and Molecular Cell Biology, University Köln Medical Center, Köln

\section{Further members of the Examination Board}

Prof. Dr. Sigrid Hoyer-Fender

Department of Developmental Biology GZMB, Johann-Friedrich-BlumenbachInstitute for Zoology and Anthropology, Georg-August-University Göttingen

Prof. Dr. Ernst Wimmer

Department of Developmental Biology GZMB, Johann-Friedrich-BlumenbachInstitute for Zoology and Anthropology, Georg-August-University Göttingen

Prof. Dr. Steven Johnsen

Clinic for General, Visceral and Pediatric Surgery, University Medical Center, Göttingen

Date of the oral examination: 
Herewith I declare, that I prepared the Dissertation

"CHARGE syndrome: candidate genes and pathogenesis"

on my own and with no other sources and aids than quoted.

Göttingen, August 20 ${ }^{\text {th }}, 2014$

Yvonne Schulz 
Dedicated to my family 


\section{Acknowledgements}

I would like to express my sincere gratitude to my doctor father Prof. Dr. med. Dr. h.c. Wolfgang Engel for the opportunity to compile my $\mathrm{PhD}$ study in the Institute of Human Genetics. I am overgrateful for the valuable scientific discussions and his encouragement during my PhD study.

I would like to thank the members of my thesis committee Prof. Dr. Andreas Wodarz and Prof. Dr. Ahmed Mansouri for their readiness to invest their time and for the valuable input during our meetings.

I would like to express my deep gratitude to my supervisor PD Dr. Silke Pauli for her unquestioning support, patience and trust and excellent guidance during the entire process of my $\mathrm{PhD}$ project. I am thankful for the facilitation to work in such interesting field of research.

Moreover, I thank Prof. Dr. Sigrid Hoyer-Fender, Prof. Dr. Ernst Wimmer and Prof. Dr. Steven Johnsen, who kindly agreed to evaluate my dissertation and participate in examination.

I sincerely thank our collaborators, especially Prof. Dr. Annette Borchers and Dr. Peter Wehner.

I would particularly like to thank the staff of the Goettingen Graduate School for Neurosciences, Biophysics and Molecular Biosciences (GGNB) and my doctoral program "Genes and Development" for the excellent organisation and financial support by the grant of the bridging fund as well as the Deutsche Forschungsgemeinschaft (DFG) for financing my $\mathrm{PhD}$ study.

Special thanks go to Johanna Mänz, who always supported my work with great assistance.

Further, I would like to thank the staff of animal keeper for excellent animal care.

I would like to thank my colleagues Johanna Mänz, Luisa Freese, Jessica Nolte, Nadine Mellies, Stefanie Heumüller, Tserendulam Batsukh, Krzysztof Wieczerzak and all other members of the Human Genetic Institute for their advice and support, friendship and great working atmosphere. 
Herewith, I would like to express my heartfelt gratitude to my family, especially to my parents who always supported me with their trust and love. I wish my dad could share this special moment of my life with me...

Thank you to Benjamin Brauer and all my friends who accompanied me through this part of my life journey. Because of you this time became an unforgettable one and will always have a special place in my heart!

Thank you so much! 


\section{Table of content}

Table of content............................................................................................................................

List of figures......................................................................................................................VI

List of tables...............................................................................................................VIII

Abbreviations................................................................................................................................X

Nomenclature........................................................................................................................XX

Summary..........................................................................................................................XXI

$1 \quad$ Introduction ............................................................................................................ 1

1.1 The chromodomain helicase DNA-binding protein 7 …............................. 1

1.2 CHD7 exists in large multi-subunit complexes............................................ 1

1.3 CHARGE syndrome .......................................................................... 3

1.4 Neural crest cells - the explorer of the vertebral embryo ………………….... 4

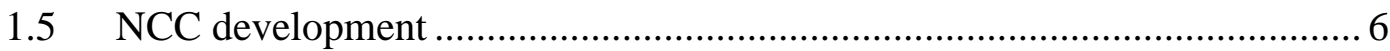

1.6 NCC guidance and signalling factors ...........................................................

1.7 Xenopus laevis as a model organism for studying NCC development.......... 9

1.8 The mouse as a model organism and its advantages ................................... 10

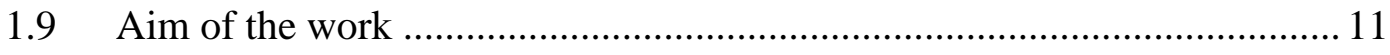

$2 \quad$ Materials and methods..................................................................................... 13

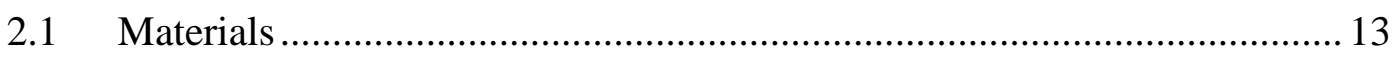

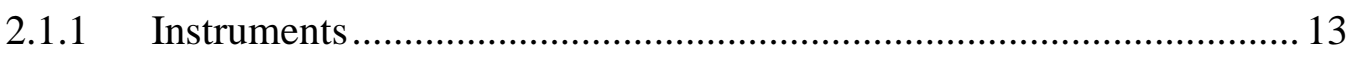

2.1.2 Consumable materials ..................................................................... 15

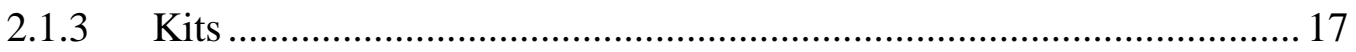

2.1.4 Ready to use buffers and mediums ................................................. 17

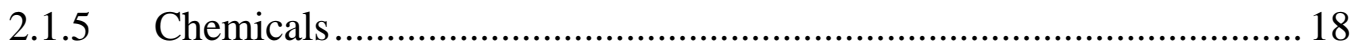

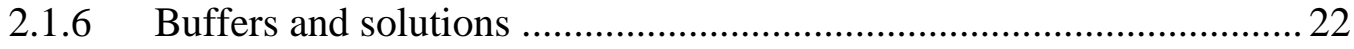

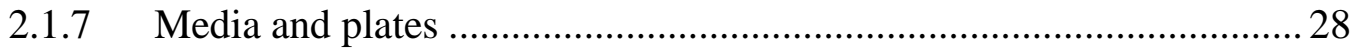

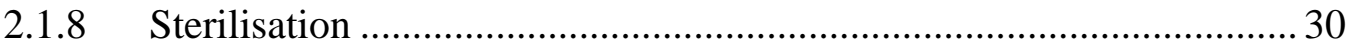




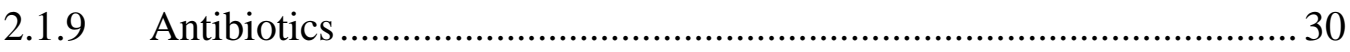

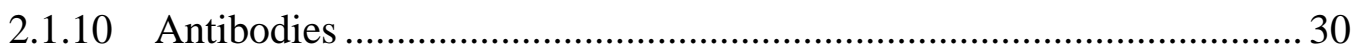

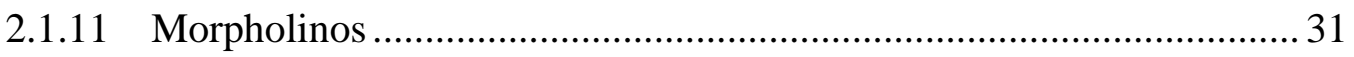

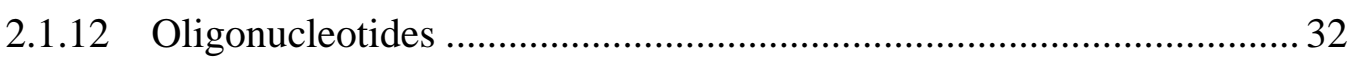

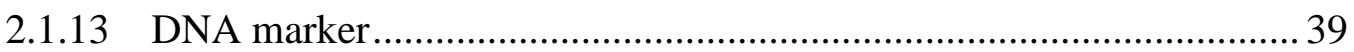

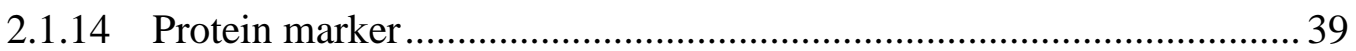

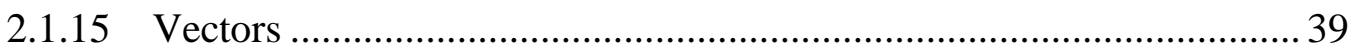

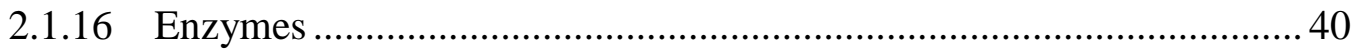

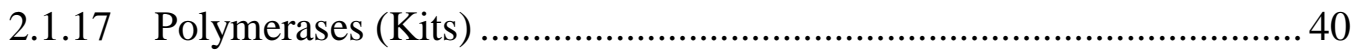

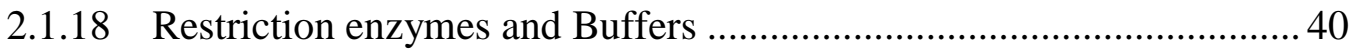

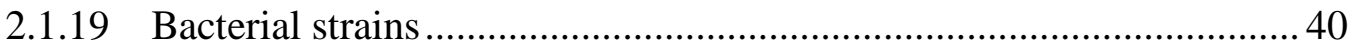

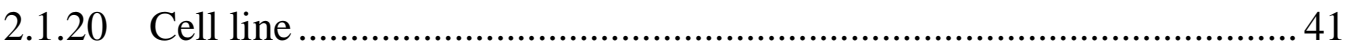

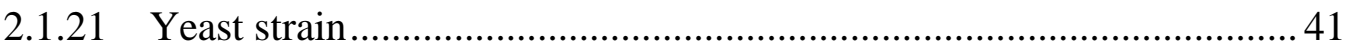

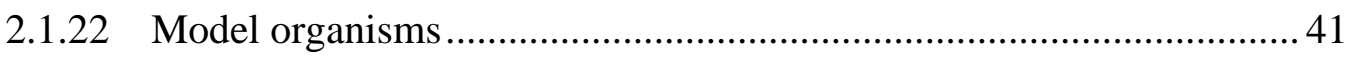

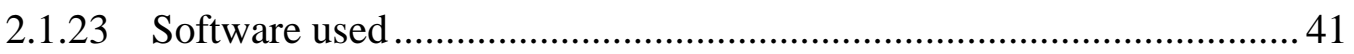

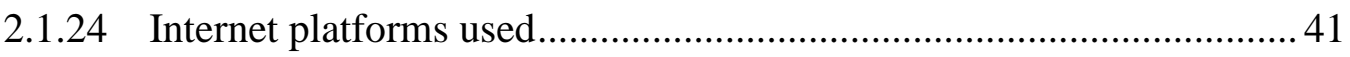

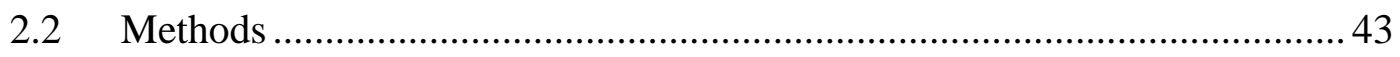

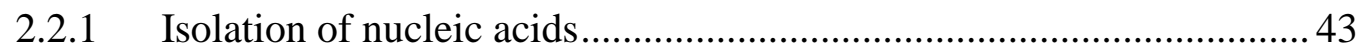

2.2.1.1 Isolation of genomic DNA for genotyping of mice .................... 43

2.2.1.2 Isolation of plasmid DNA from bacteria .................................. 43

2.2.1.3 RNA isolation from mouse embryos ...................................... 45

2.2.2 Determination of nucleic acid concentration .................................. 46

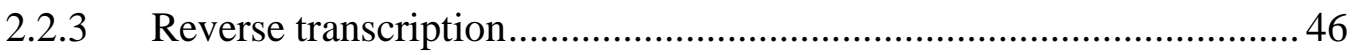

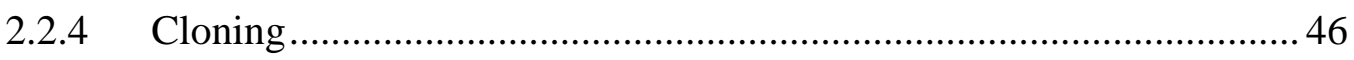

2.2.4.1 Restriction digestion of plasmid DNA................................... 47

2.2.4.2 Ligation of DNA fragments into plasmids ............................... 47

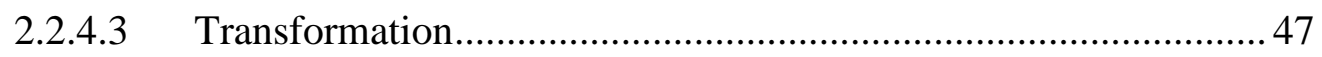

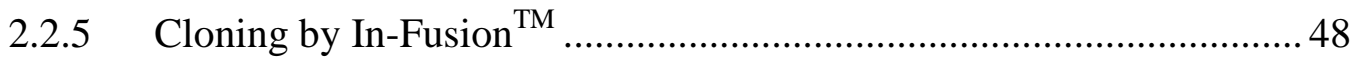




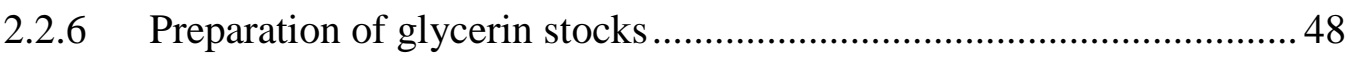

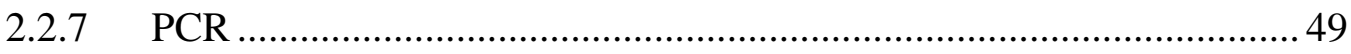

2.2.7.1 Touchdown PCR .................................................................... 53

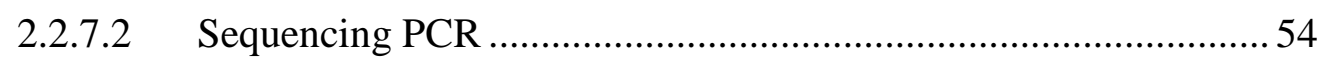

2.2.8 Quantitative real-time PCR and data analysis................................. 55

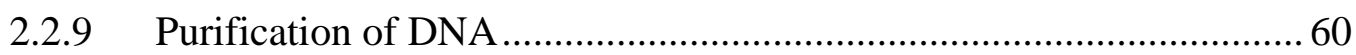

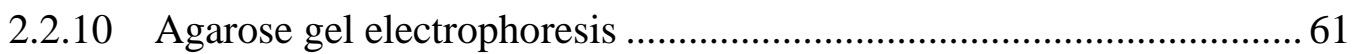

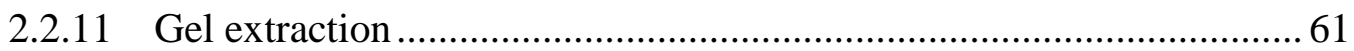

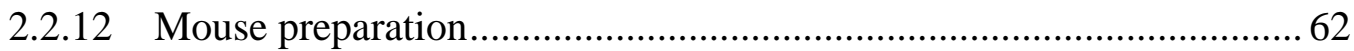

2.2.13 Fixation and dehydration of embryos for paraffin embedding ............ 62

2.2.14 Preparation of paraffin sections of mouse embryos ........................... 63

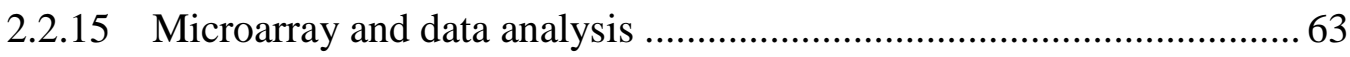

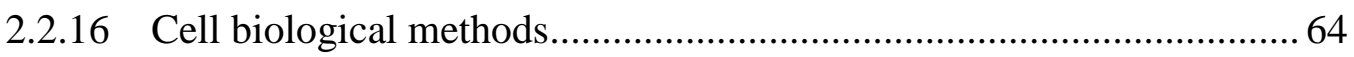

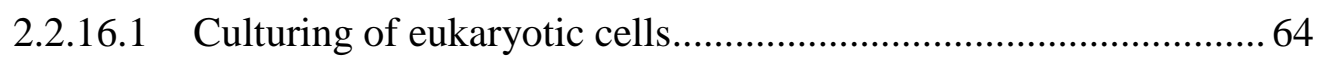

2.2.16.2 Cryopreservation and revitalisation of eukaryotic cells .............. 64

2.2.16.3 Transfection of eukaryotic cells with plasmids .......................... 65

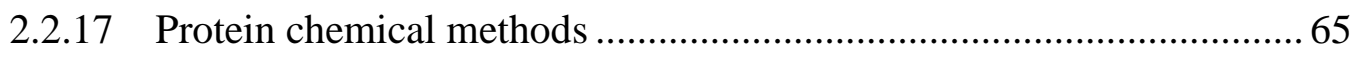

2.2.17.1 Protein isolation from HeLa cells ............................................... 65

2.2.17.2 Measurement of protein concentration ...................................... 66

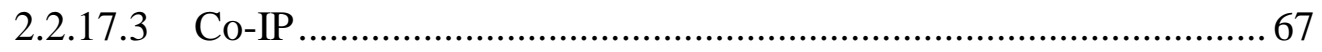

2.2.17.4 SDS polyacrylamide gel electrophoresis ................................... 68

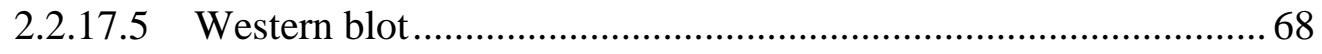

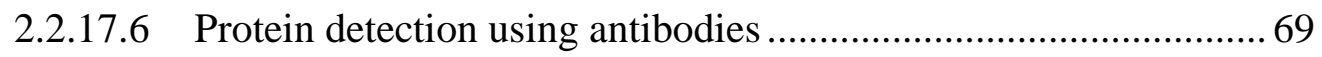

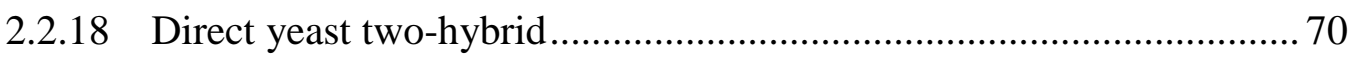

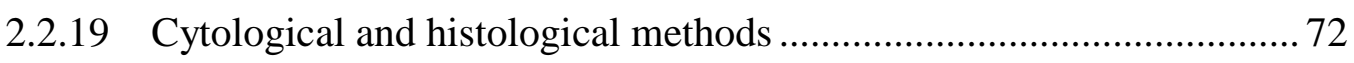

2.2.19.1 Immunocytochemistry with eukaryotic cells ............................ 72

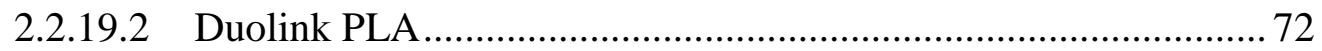

2.2.19.3 Immunohistochemistry (3,3'-Diaminobenzidine (DAB) staining) 74 
2.2.21 Molecular biological methods concerning the model organism Xenopus laevis 76

2.2.21.1 In vitro transcription of sense RNA ...................................... 76

2.2.21.2 Purification of sense RNA ......................................................... 76

2.2.21.3 In vitro transcription of labeled anti-sense RNA ....................... 77

2.2.21.4 Purification of labeled anti-sense RNA .................................... 77

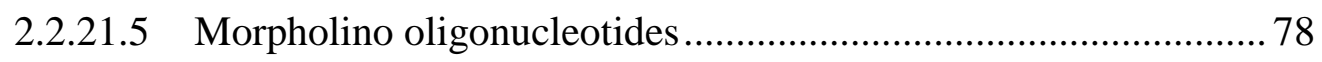

2.2.21.6 Preparation of Xenopus laevis testis and fertilisation of oocytes.. 78

2.2.21.7 Microinjection of Xenopus laevis embryos and culture ............... 78

2.2.21.8 Fixation of injected Xenopus laevis embryos and X-gal staining. 79

2.2.21.9 Whole mount in situ hybridisation.......................................... 79

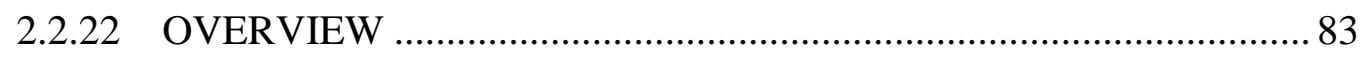

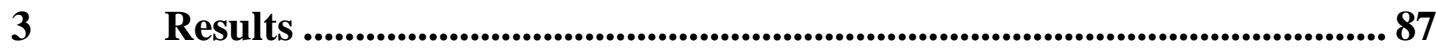

3.1 CHD7 interacts with components of the WAR complex ........................... 87

3.2 CHD7 and the members of the WAR complex are co-localised in the nucleus

3.3 CHD7 shows no direct interaction with WAR complex members 92

3.4 Genome-wide expression analysis demonstrates a misregulation of NCC guidance genes in case of CHD7 loss of function

3.5 Expression analysis by RT-qPCR confirms the results of the microarray .. 97

3.6 There is no gender specific effect on the expression of Sema3a, Sema3d, Epha3, Trp53bp2, Sox10 and Gfra2 in Whirligig mouse embryos....

3.7 CHD7 and SEMA3D show a similar expression pattern in mouse embryos 101

3.8 Results of SEMA3A and SEMA3D mutation screens in patients with CHARGE syndrome 103

3.9 Knockdown of Chd7 causes alteration in sema3a expression in Xenopus laevis 105 
3.10 The Chd7 MO phenotype was successfully rescued by human CHD7 RNA 108

3.11 Knockdown of Sema3a and Sema3d causes migration defects of NCCs in Xenopus laevis

3.12 Double knockdown of Chd7 and Sema3a or Sema3d has no clear synergistic effect on twist expression in Xenopus laevis

4

Discussion

4.1 Short summary

4.2 CHARGE syndrome shows phenotypical overlap with other syndromes 122

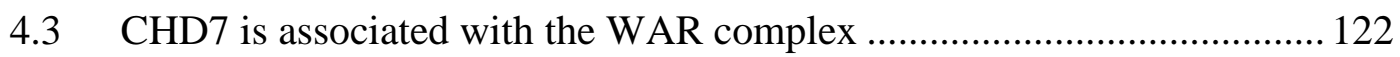

4.4 CHD7 regulates genes required for proper NCC development ................. 124

4.5 Chd7 regulates sema3a expression and NCC induction and migration in

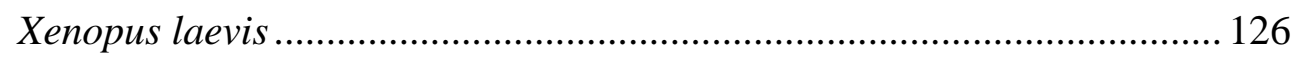

4.6 SEMA3A and SEMA3D seem to play a role in the pathogenesis of CHARGE syndrome ................................................................... 127

4.7 Semaphorins might act as modifier in CHARGE syndrome.................... 128

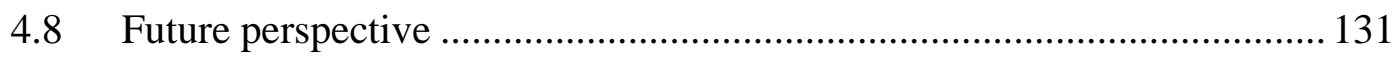

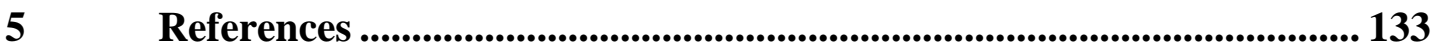

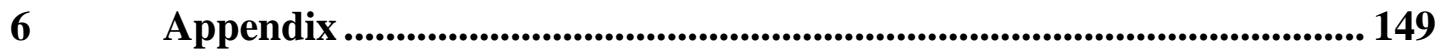

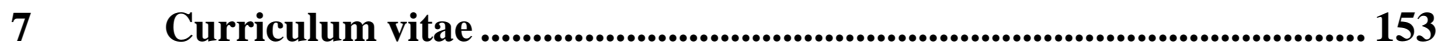




\section{List of figures}

Figure 1: Possible composition of the MLL methyltransferase complex................... 3

Figure 2: Schematic overview of induction and delamination of neural crest cells... 4

Figure 3: Differentiation of neural crest cells. ...................................................... 6

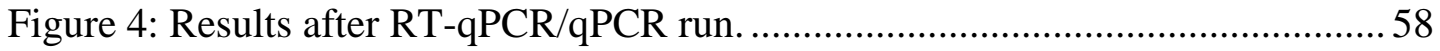

Figure 5: Co-immunoprecipitation experiments with HeLa cells............................ 88

Figure 6: Protein localisation after immunocytochemistry performed with HeLa cells.

Figure 7: Analysing protein interactions in HeLa cells using the Duolink PLA

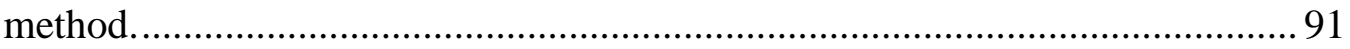

Figure 8: Results of the direct yeast two-hybrid assay. .......................................... 93

Figure 9: Schematic representation of the CHD7 constructs for yeast two-hybrid

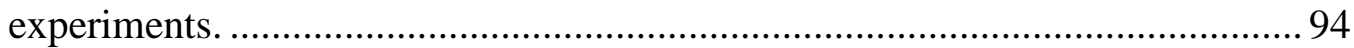

Figure 10: Heatmap of differentially expressed genes found by microarray............. 96

Figure 11: Results of the gene expression analysis by RT-qPCR........................... 98

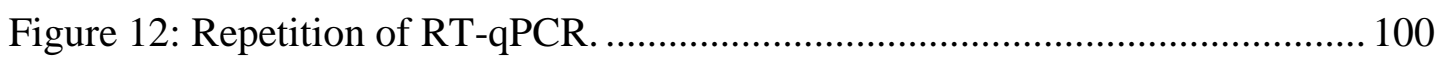

Figure 13: CHD7 and SEMA3D expression analysis using paraffin sections of mouse

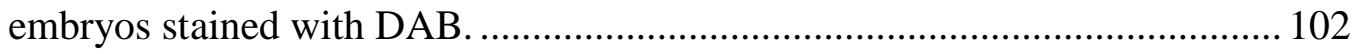

Figure 14: Results of the SEMA3A mutation screen.............................................. 104

Figure 15: Results of the SEMA3D mutation screen............................................. 105

Figure 16: Sema3a expression analysis by whole mount in situ hybridisation after

Chd7 knockdown in Xenopus laevis (neurula stage 20). 106

Figure 17: Sema3a expression after Chd7 knockdown in Xenopus laevis (tailbud stage 27).

Figure 18: Rescue of the Chd7 MO phenotype in Xenopus laevis (neurula stage 21).

Figure 19: Repetition of the rescue of the Chd7 MO phenotype in Xenopus laevis (neurula stage 20).

Figure 20: Twist expression analysis after knockdown of Chd7, Sema3a or Sema3d in Xenopus laevis (neurula stage 21).

Figure 21: Effect of Chd7, Sema3a or Sema3d knockdown on NCCs (twist expression) in Xenopus laevis (tailbud stage 26).

Figure 22: Effect on twist expression after Chd7 knockdown, Chd7/Sema3a and Chd7/Sema3d double knockdown in Xenopus laevis. 
Figure 23: Effect on the NCC marker twist after Chd7 knockdown, Chd7/Sema3a and Chd7/Sema3d double knockdown in Xenopus laevis............................. 119 


\section{List of tables}

Table 1: Function of factors involved in early migration and guidance of neural crest cells.

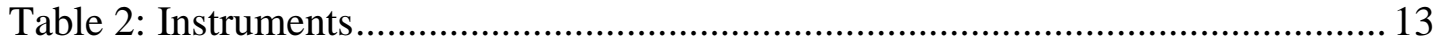

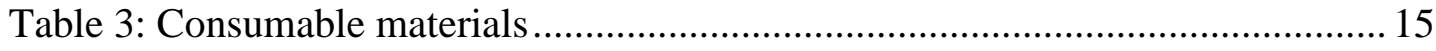

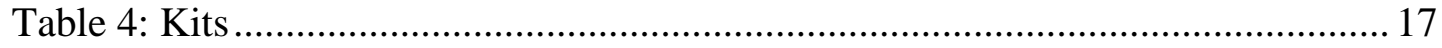

Table 5: Ready to use buffers and mediums .......................................................... 18

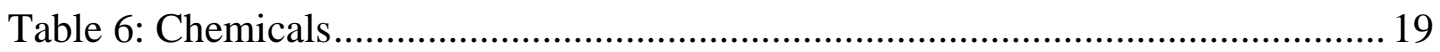

Table 7: Agarose gel electrophorese .................................................................. 22

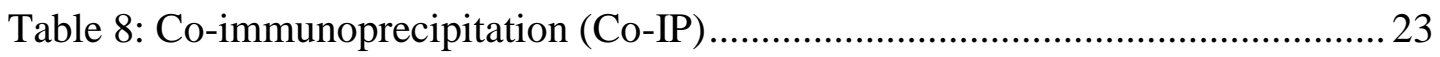

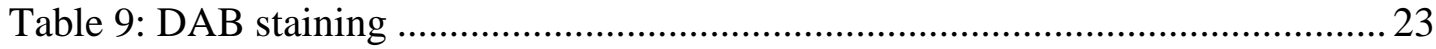

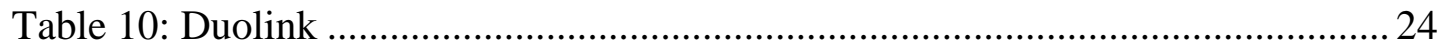

Table 11: Polymerase chain reaction (PCR) …...................................................... 24

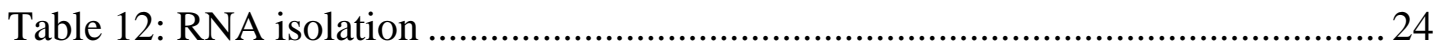

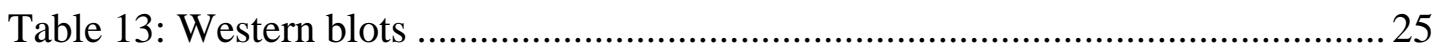

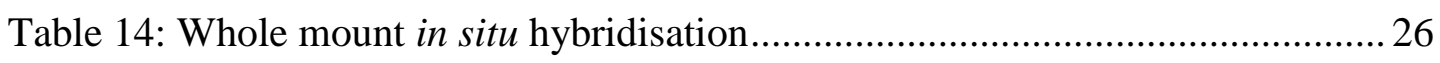

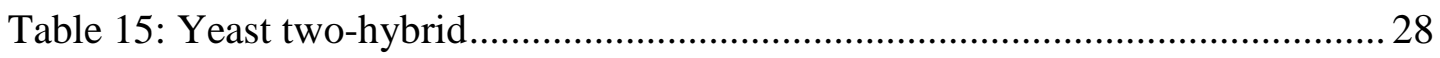

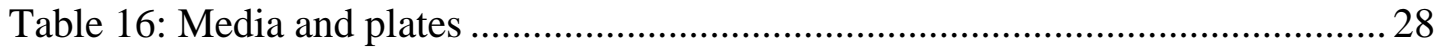

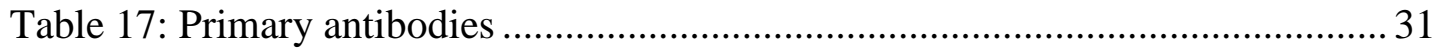

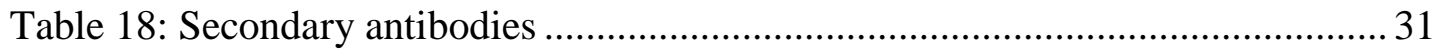

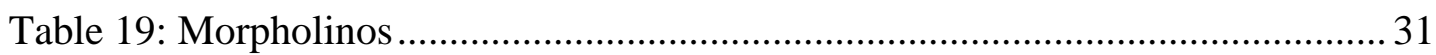

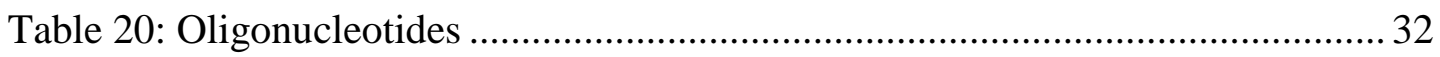

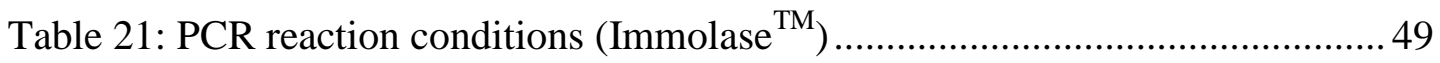

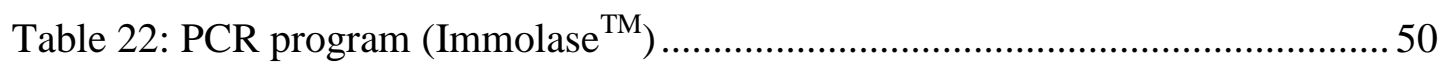

Table 23: PCR reaction conditions (peqGOLD Pwo) ...............................................50

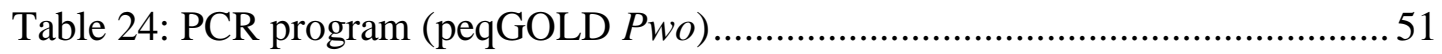

Table 25: PCR reaction conditions (PfuUltra ${ }^{\mathrm{TM}}$ High-Fidelity) ................................ 52

Table 26: PCR program (PfuUltra ${ }^{\mathrm{TM}}$ High-Fidelity) ................................................ 52

Table 27: PCR reaction conditions (Platinum ${ }^{\circledR}$ Taq) .............................................. 53

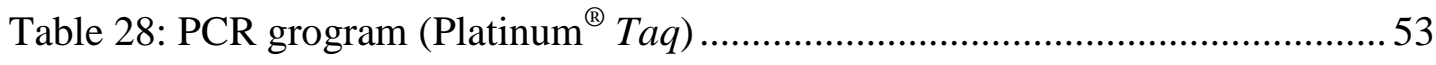

Table 29: PCR reaction conditions (Touchdown).................................................... 54

Table 30: PCR program (Touchdown) .................................................................... 54

Table 31: PCR reaction conditions (sequencing) …............................................... 55

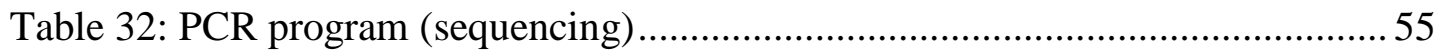




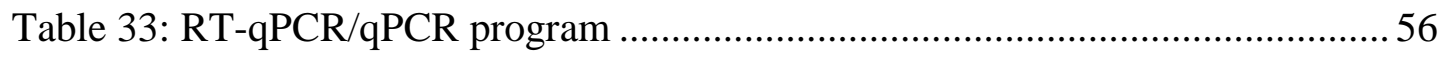

Table 34: reaction conditions (RT-qPCR/qPCR) ....................................................5

Table 35: Terms used in Allelic Discrimination Analysis......................................... 57

Table 36: Reagent volumes for different packed cell volume ................................... 66

Table 37: Reaction conditions (in vitro transcription of labeled anti-sense RNA).... 77

Table 38: Proteinase K treatment of Xenopus laevis embryos.................................. 80

Table 39: Microarray gene expression analysis .................................................. 149 


\section{Abbreviations}

A

aa

Aba

$\mathrm{ad}$

Ade

ADE2

ASH $2 L$

ATP

b

BAF

BamHI

bp

BCIP

$\mathrm{BMB}$

BRK

BSA

C

c

c.

${ }^{\circ} \mathrm{C}$

$\mathrm{CaCl}_{2}$

cc

cDNA

CHAPS

CHD

CHD7

CHD8

chp

$\mathrm{Cl}$

$\mathrm{cm}$ adenine, alanine

amino acid

aureobasidin A

to, up to

adenine

adenine promotor

ash2 (absent, small, or homeotic)-like

adenosine triphosphate

constant (interception of y-axis), branchial

Brahma-associated factor

Bacillus amyloliquefaciens $\mathrm{H} \mathrm{I}$

base pair

5-Brom-4-chlor-3-indolylphosphate

Boehringer Mannheim Blocking Reagent

Brahma and Kismet

bovine serum albumin

cytosine

molar concentration, cochlea

cDNA reference sequence

degree Celsius

calcium chloride

cartilage condensation being primordium of vertebral body

complementary deoxyribonucleic acid

3-[(3-Cholamidopropyl)dimethylammonio]-1propanesulfonate

chromodomain helicase DNA binding protein

chromodomain helicase DNA binding protein 7

chromodomain helicase DNA binding protein 8

choroid plexus differentiating from roof of

fourth ventricle

chloride

centimetre 


\begin{tabular}{|c|c|}
\hline $\mathrm{cm}^{2}$ & square centimetre \\
\hline Co-IP & co-immunoprecipitation \\
\hline $\mathrm{CO} \mathrm{MO}$ & control Morpholino \\
\hline $\mathrm{cp}$ & cartilage primordium of body of vertebra \\
\hline $\mathrm{CR}$ & conserved region \\
\hline $\mathrm{Ct}$ & threshold cycle \\
\hline C-terminus & carboxy-terminus \\
\hline CTP & cytidine triphosphate \\
\hline Cy3 & cyanine dye 3 \\
\hline DAB & 3,3'-Diaminobenzidine \\
\hline DAPI & 4',6-diamidino-2-phenylindole \\
\hline dATP & deoxyadenosine triphosphate \\
\hline dCTP & deoxycytidine triphosphate \\
\hline $\mathrm{ddH}_{2} \mathrm{O}$ & double distilled water \\
\hline ddNTPs & dideoxynucleotide triphosphates \\
\hline DEPC & diethylpyrocarbonate \\
\hline dGTP & deoxyguanosine triphosphate \\
\hline DMEM & Dulbecco's Modified Eagle Medium \\
\hline DMSO & dimethyl sulfoxid \\
\hline DNA & deoxyribonucleic acid \\
\hline DNaseI & deoxyribonuclease I \\
\hline dNTP & deoxynucleotide triphosphate \\
\hline DO supplement & dropout supplement \\
\hline DPBS & dulbecco's phosphate buffered saline \\
\hline DTT & 1,4-Dithiothreitol \\
\hline dTTP & desoxythymidin triphosphate \\
\hline $\mathrm{E}$ & efficiency, embryonic stage \\
\hline E. coli & Escherichia coli \\
\hline EcoRI & Escherichia coli. strain R I \\
\hline e-cup & eppendorf reaction vessel \\
\hline EDTA & ethylene diamine tetraacetic acid \\
\hline e.g. & exempli gratia (for example) \\
\hline EGTA & ethylene glycol tetraacetic acid \\
\hline EMT & epithelial-to-mesenchymal transition \\
\hline
\end{tabular}




\begin{tabular}{|c|c|}
\hline ENU & N-ethyl-N-nitrosourea \\
\hline Eph & Ephrin \\
\hline Epha3 & Eph receptor $A 3$ \\
\hline Epha5 & Eph receptor $A 5$ \\
\hline Epha7 & Eph receptor $A 7$ \\
\hline ESP & Exome Sequencing Project \\
\hline et al. & et alteri (and others) \\
\hline $\mathrm{EtOH}$ & ethanol \\
\hline $\mathrm{FC}$ & fold change \\
\hline FCS & fetal calf serum \\
\hline FDR & false discovery rate \\
\hline Fig. & figure \\
\hline FITC & fluorescein isothiocyanate \\
\hline Foxd3 & forkhead box D3 \\
\hline fv & forth ventricle \\
\hline g & gram, constant of gravitation, ganglia \\
\hline G & guanine \\
\hline GenRE & genome research environment \\
\hline \multirow[t]{2}{*}{ Gfra2 } & glial cell line derived neurotrophic factor family \\
\hline & receptor alpha 2 \\
\hline GnRH & gonadotropin-releasing hormone \\
\hline G-protein & guaninnucleotide-binding protein \\
\hline \multirow[t]{2}{*}{ GSF } & Forschungszentrum für Umwelt und Gesundheit \\
\hline & (Gesellschaft für Strahlenforschung) \\
\hline GTP & guanosine-5'-triphosphate \\
\hline $\mathrm{H}$ & histidine \\
\hline h & heart, hour, human, hyoid \\
\hline HA-Tag & hemagglutinin Epitop marker \\
\hline hCG & human chorionic gonadotropin \\
\hline $\mathrm{HCl}$ & hydrochloric acid \\
\hline HeLa & Henrietta Lacks \\
\hline \multirow[t]{2}{*}{ HEPES } & 2-[4-(2-hydroxyethyl)piperazin-1-yl] \\
\hline & ethanesulfonic acid \\
\hline Het & heterozygous \\
\hline
\end{tabular}


hgvs

His

HIS3

H3K 4

$\mathrm{H}_{2} \mathrm{O}$

$\mathrm{H}_{2} \mathrm{O}_{2}$

Homo

HOX

HOXA2

Hprt

HRP

Hsc-70

I

IgG

IP

K

$\mathrm{kb}$

$\mathrm{KCl}$

$\mathrm{kDa}$

$\mathrm{K}_{3} \mathrm{Fe}(\mathrm{CN})_{6}$

$\mathrm{K}_{4} \mathrm{Fe}(\mathrm{CN})_{6}$

KMT2D

$\mathrm{KNO}_{3}$

L

1

lacZ

LB

LDS

Leu

LEU2

LiAc

$\log$

-LT

-LTHA human genome variation society

histidine

histidine promotor

histone 3 lysine 4

water

hydrogen peroxide

homozygous

homeobox

homeobox A2

Hypoxanthin-phosphoribosyl-transferase

horseradish peroxidase

heat shock protein cognate 70

isoleucine

Immunoglobulin G

immunoprecipitation

lysine

kilo base pair

potassium chloride

kilo Dalton

potassium ferricyanide

potassium ferrocyanide

lysine (K)-specific methyltransferase $2 D$

potassium nitrate

litre, leucine

liver

beta-D-galactosidase

lysogeny broth

lithium dodecyl sulfate

leucine

leucine promotor

lithium acetate

decadic logarithm

without leucine, tryptophan

without leucine, tryptophan, histidine, adenine 
molar

m

slope, mandibular

$\mathrm{mA}$

milliampere

MAB

maleic acid buffer

MBS

modified barth solution

MEM

MEMFA

MES

$\mathrm{MetOH}$

mg

$\mathrm{MgCl}_{2}$

$\mathrm{MgSO}_{4}$

$\mathrm{mh}$

$\min$

MIPS

MIQE

miRNA

$\mathrm{ml}$

MLL2

$\mathrm{mM}$

$\mathrm{mm}$

MO

mo

MOPS

mRNA

MscI

mv

$\mathrm{N}$

$\mathrm{NaHCO}_{3}$

$\mathrm{NaH}_{2} \mathrm{PO}_{4}$

$\mathrm{Na}_{2} \mathrm{HPO}_{4}$

Nanog 


\begin{tabular}{|c|c|}
\hline $\mathrm{NaOH}$ & Sodium hydroxide \\
\hline NBT & Nitro blue tetrazolium \\
\hline $\mathrm{NC}$ & negative control \\
\hline NCBI & National Center for Biotechnology Information \\
\hline $\mathrm{NCC}$ & neural crest cell \\
\hline NdeI & Neisseria denitrificans I \\
\hline NEAA & non-essential amino acids \\
\hline NEB & New England Biolabs \\
\hline ng & nanogram \\
\hline$N L K$ & Nemo-Like Kinase \\
\hline NM & NCBI accession number \\
\hline $\mathrm{nm}$ & nanometre \\
\hline NP & NCBI Reference Sequence for proteins \\
\hline NruI & Nocardia rubra I \\
\hline ns & not specified \\
\hline N-terminus & Amino-terminus \\
\hline Oct4 & Octamer binding transcription factor 4 \\
\hline OD & optical density \\
\hline oe & olfactory epithelium \\
\hline $\mathrm{OH}$ & hydroxy \\
\hline OMIM & Online Mendelian Inheritance in Man \\
\hline $\mathrm{P}$ & proline \\
\hline $\mathrm{p}$. & protein reference sequence \\
\hline $\mathrm{Pa}$ & pascal (unit of pressure) \\
\hline $\operatorname{Pax} 2$ & paired box gene 2 \\
\hline PBAF & $\begin{array}{l}\text { polybromo- and BRG1-associated factor } \\
\text { containing complex }\end{array}$ \\
\hline PBS & phosphate buffered saline \\
\hline PBST & phosphate buffered saline Tween-20 \\
\hline pCMV & plasmid cytomegalovirus \\
\hline PCR & polymerase chain reaction \\
\hline$P d g f$ & platelet derived growth factor \\
\hline$P d g f c$ & platelet derived growth factor $C$ \\
\hline PEG & polyethylene glycol \\
\hline
\end{tabular}




\begin{tabular}{|c|c|}
\hline $\mathrm{pH}$ & potentia hydrogenii \\
\hline Pitx2 & paired-like homeodomain 2 \\
\hline PLA & proximity ligation assay \\
\hline pmol & picomol \\
\hline PMSF & Phenylmethanesulfonylfluoride \\
\hline PolyPhen2 & $\begin{array}{l}\text { Polymorphism Phenotyping v2 (prediction } \\
\text { program) }\end{array}$ \\
\hline$P P A R Y$ & $\begin{array}{l}\text { peroxisome proliferator activated receptor } \\
\text { gamma }\end{array}$ \\
\hline pre-mRNA & premature messenger ribonucleic acid \\
\hline Ptw & phosphate buffered saline with Tween 20 \\
\hline PVDF & polyvinyliden fluoride \\
\hline Q & glutamine \\
\hline q & long arm of chromosome \\
\hline qPCR & quantitative real-time polymerase chain reaction \\
\hline $\mathrm{R}$ & arginine \\
\hline $\mathrm{R}^{2}$ & coefficient of determination \\
\hline$R B B P 5$ & retinoblastoma binding protein 5 \\
\hline $\mathrm{R}^{\mathrm{n}}$ & normalised reporter fluorescence \\
\hline RNA & ribonucleic acid \\
\hline Robo & roundabout \\
\hline ROX & 6-carboxy-x-rhodamine \\
\hline rpm & revolutions per minute \\
\hline RT-qPCR & $\begin{array}{l}\text { reverse transcription-quantitative real-time } \\
\text { polymerase chain reaction }\end{array}$ \\
\hline$S$ & serine \\
\hline $\mathrm{s}$ & somites \\
\hline SANT & $\begin{array}{l}\text { Switching-defective protein 3, Adaptor 2, } \\
\text { Nuclear receptor corepressor, Transcription } \\
\text { factor IIIB }\end{array}$ \\
\hline $\mathrm{sb}$ & $\begin{array}{l}\text { segmental bronchus within accessory lobe of } \\
\text { right lung }\end{array}$ \\
\hline Sdha & succinate dehydrogenase complex, subunit $A$ \\
\hline
\end{tabular}


SDS

sec

Sema3a

Sema3c

Sema3d

Sema3e

Sema4d

SET1

SETDB1

SfiI

SIFT

Slitrk1

Slitrk6

Slug/snail

sm

S.O.C.

Sox 2

Sox 9

Sox10

SSC

SWI2/SNF2

$\mathrm{T}$

Tab.

$T b p$

TBST

TE

Tris sodium-dodecyl-sulfate, sequence-detection software

second

sema domain, immunoglobulin domain (Ig), short basic domain, secreted, (semaphorin) $3 A$ sema domain, immunoglobulin domain (Ig), short basic domain, secreted, (semaphorin) $3 C$ sema domain, immunoglobulin domain (Ig), short basic domain, secreted, (semaphorin) 3D sema domain, immunoglobulin domain (Ig), short basic domain, secreted, (semaphorin) $3 E$ sema domain, immunoglobulin domain (Ig), short basic domain, secreted, (semaphorin) 4D histone lysine methyltransferase Set1 SET domain, bifurcated 1 Streptomyces fimbriatus I sorts intolerant from tolerant (prediction program)

SLIT and NTRK-like family, member 1 SLIT and NTRK-like family, member 6 Zinc finger protein Slug/ snail family zinc finger splanchic mesoderm super optimal broth SRY (sex determining region Y)-box 2 SRY (sex determining region Y)-box 9 SRY (sex determining region Y)-box 10 saline-sodium citrate SWItch/Sucrose NonFermentable thymine, threonine table

TATA box binding protein

Tris-buffered saline and Tween 20 Tris-EDTA

2-amino-2-hydroxymethyl-propane-1,3-diol 


\begin{tabular}{|c|c|}
\hline $\operatorname{Trp}$ & tryptophan \\
\hline TRP1 & tryptophan promotor \\
\hline $\operatorname{Trp} 53 b p 2$ & $\begin{array}{l}\text { transformation related protein } 53 \text { binding } \\
\text { protein } 2\end{array}$ \\
\hline Trx & trithorax \\
\hline tv & right telencephalic vesicle \\
\hline twist & basic helix-loop-helix transcription factor twist \\
\hline $\mathrm{U}$ & unit \\
\hline UTP & uridine-5'-triphosphate \\
\hline UV & ultraviolet light \\
\hline V & volt, valine \\
\hline Vegf & vascular endothelial growth factor \\
\hline$V e g f c$ & vascular endothelial growth factor $C$ \\
\hline Vol & volume \\
\hline VP & vaginal plug \\
\hline $\mathrm{v} / \mathrm{v}$ & volume/volume \\
\hline $\mathrm{W}$ & tryptophan \\
\hline WAR & WDR5, ASH2L, RBBP5 \\
\hline WDR5 & WD repeat domain 5 \\
\hline Whi & Whirligig \\
\hline WMIISH & whole mount in situ hybridisation \\
\hline WT & wild-type \\
\hline $\mathrm{w} / \mathrm{v}$ & weight/volume \\
\hline $\mathrm{x}$ & times concentrated stock \\
\hline $\mathrm{X}-\mathrm{Gal}$ & $\begin{array}{l}\text { 5-bromo-4-chloro-3-indolyl- } \beta \text {-D- } \\
\text { galactopyranoside }\end{array}$ \\
\hline XhoI & Xanthomonas holcicola I \\
\hline X-Ray & roentgen radiation \\
\hline $\mathrm{Y} 2 \mathrm{H}$ & yeast two-hybrid \\
\hline YNB & yeast nitrogen base \\
\hline I & roman numeral (1) \\
\hline II & roman numeral (2) \\
\hline III & roman numeral (3) \\
\hline$\alpha$ & alpha \\
\hline
\end{tabular}


$\beta$

X

$\%$

$\mu \mathrm{g}$

$\mu 1$

$\Delta$

$\infty$

(

TM

$>$

$<$

$\sim$ beta

gamma

percent

microgram

microlitre

delta

infinity

registered Trade Mark

unregistered Trade Mark

greater than

less than

tilde (informally means approximately) 


\section{Nomenclature}

In this work mutations were described using the nomenclature according to the human genome variation society (hgvs) (den Dunnen and Antonarakis, 2000; HGVS, 2013). Genes and proteins stated in this work followed the guidelines according to the HUGO Gene Nomenclature Committee (HGNC) for human (Wain et al., 2002), the Mouse Genome Informatics Database (MGI) for mouse (MGI, 2011) and Xenbase Gene Nomenclature Guidelines for Xenopus laevis (Xenbase, 2013).

The abbreviation qPCR was used for quantitative real-time PCR and RT-qPCR for reverse transcription-qPCR as proposed by Bustin et al. (2009). 


\section{Summary}

CHARGE syndrome is a complex malformation syndrome affecting several organ systems like the central nervous system, ear, eye and heart. Heterozygous loss of function mutations in CHD7, a conserved chromatin remodelling enzyme, are responsible to cause about two-thirds of CHARGE syndrome cases. CHD7 is present in large multi-subunit complexes regulating gene transcription in a time and cell type specific manner.

In this work, it was shown by co-immunoprecipitation and Duolink proximity ligation assay that CHD7 interacts with the WAR complex members WDR5, ASH2L and RBBP5. Additional direct yeast two-hybrid experiments revealed that CHD7 is most likely associated to this complex via its direct interaction partner CHD8.

The WAR complex is one "core" complex of the methyltransferase KMT2D. Mutations in $K M T 2 D$ are the main cause of Kabuki syndrome, a developmental disorder, showing a remarkable phenotypic overlap to features present in CHARGE syndrome. The results of this work demonstrate a possible link of CHD7 and KMT2D to the same regulatory process of chromatin remodelling and chromatin modification, which might explain the phenotypic overlap of CHARGE syndrome and Kabuki syndrome.

It was proposed that CHARGE syndrome belongs to the neurocristopathies. Recent studies demonstrated that CHD7 is required for the formation of multipotent neural crest cells. However, little is known about CHD7 target genes in the process of neural crest cell development. Within this work a genome-wide microarray analysis was performed with embryos (wild-type $\left(C h d 7^{+/+}\right)$, heterozygous $\left(C h d 7^{\text {Whi/++}}\right)$ and homozygous $\left(C h d 7^{\text {Whi/Whi}}\right)$ ) of the Whirligig mouse line which carries a nonsense mutation in the $C h d 7$ gene. 98 genes were identified to be differentially expressed comparing homozygous $\left(C h d 7^{W h i / W h i}\right)$ to wild-type $\left(C h d 7^{+/+}\right)$embryos. In fact, many of these genes are involved in the development of neural crest cells, for example, the specification of neural crest cells, epithelial-to-mesenchymal-transition and guidance of migrating neural crest cells. In this work it was demonstrated that the regulatory effect of Chd7 on Sema3a, a secreted signalling molecule known to have chemorepulsive properties for axons and to guide migrating neural crest cells, is conserved in mouse and Xenopus laevis. Further, a regulatory effect on neural crest cell migration in Xenopus laevis was exposed for Sema3a and Sema3d. 
In this work three non-synonymous mutations were identified in $C H D 7$ negative CHARGE patients within the SEMA3A gene and the SEMA3D gene, respectively. SEMA3A mutations are known to be involved in the pathogenesis of Kallmann syndrome, a genetic disorder, which represents the mild phenotypic end of CHARGE syndrome. It is assumed that SEMA3A and SEMA3D act as modifiers contributing to a more severe phenotype in CHARGE patients if they are mutated. This would explain the high inter- and intra-familial variability observed in CHARGE syndrome. In general, the results discovered in this work help to better understand the pathogenic mechanism behind CHARGE syndrome. 


\section{Introduction}

\subsection{The chromodomain helicase DNA-binding protein 7}

CHD7 (Chromodomain helicase DNA-binding protein 7) belongs to the CHD (Chromodomain helicase DNA-binding protein) family of ATP (adenosine triphosphate)-dependent chromatin remodelling enzymes which regulate gene transcription (Kim et al., 2008b). In human the CHD family consists of nine members which share two chromatin domains located at the $\mathrm{N}$-terminus and a SWI2/SNF2-like ATPase/helicase domain (Woodage et al., 1997; Flaus et al., 2006; Flanagan et al., 2007). According to domain and sequence similarities the nine members of the CHD family are divided into three subgroups. CHD7 belongs to subgroup III which is characterised by three conserved regions (CR1-3), a SANT (Switching-defective protein 3, Adaptor 2, Nuclear receptor corepressor, Transcription factor IIIB) domain and two BRK (Brahma and Kismet) domains located at the C-terminal region (J. A. Hall and Georgel, 2007; Marfella and Imbalzano, 2007). CHD7 is located on chromosome 8 (8q12) and it consists of 38 exons with a genomic size of $188 \mathrm{~kb}$ (Vissers et al., 2004). CHD7 is highly conserved in different species, such as mouse, chick, zebrafish, Xenopus laevis and others where orthologs were identified (Bosman et al., 2005; Aramaki et al., 2007; Bajpai et al., 2010).

\subsection{CHD7 exists in large multi-subunit complexes}

It is known that chromatin remodelling enzymes form multi-subunit complexes which regulate gene transcription in a certain way depending on the composition of proteins within these complexes (Mohrmann and Verrijzer, 2005; Trotter and Archer, 2008; Ho et al., 2009b). Schnetz et al. (2010) showed in mouse embryonic stem cells that CHD7 binds OCT4 (octamer binding transcription factor 4), SOX2 (SRY (sex determining region Y)-box 2), NANOG (Nanog homeobox) and the enhancer binding protein P300. Another co-localisation of CHD7 was described by Bajpai et al. (2010), who found CHD7 to be associated with members of the remodelling BAF/PBAF complex (Brahma-associated factor/Polybromo- and BRG1-associated factor containing complex) in human neural crest like cells. Takada et al. (2007) 
described the involvement of a complex consisting of CHD7, PPARy (peroxisome proliferator activated receptor gamma), NLK (Nemo-Like Kinase) and SETDB1 (SET domain, bifurcated 1) in the process of adipogenesis and osteoblastogenesis in mouse bone marrow mesenchymal progenitor cells. Furthermore, it was shown by our group that CHD7 interacts indirectly and directly with the chromatin remodeler CHD8 (Chromodomain helicase DNA-binding protein 8) (Batsukh et al., 2010). Yates et al. (2010) demonstrated that Chd8 is associated with the WAR complex (WDR5 (WD repeat domain 5), ASH2L (absent, small, or homeotic-like) and RBBP5 (retinoblastoma binding protein 5)). Another factor also associated with the WAR complex is the methyltransferase KMT2D (lysine (K)-specific methyltransferase 2D) (OMIM 602113). An alternative symbol is MLL2 (mixed lineage leucemia 2). KMT2D belongs to the SET1 (histone lysine methyltransferase Set1) family and is responsible for histone 3 lysine 4 (H3K4) di- and trimethylation (Song JJ, 2008). Members of the SET1 family of enzymes are commonly associated with multi-subunit complexes which are important for the activation of the methyltransferase (Miller et al., 2001; Yokoyama et al., 2004; Wysocka et al., 2005; Steward et al., 2006; Ernst and Vakoc, 2012). One "core" complex consists of WDR5, ASH2L and RBBP5 (Steward et al., 2006; Song JJ, 2008; Yates et al., 2010). Yates et al. (2010) described the MLL (mixed lineage leucemia) associated WAR complex as a positive regulator of HOXA2 (homeobox A2) transcription. Furthermore, it is known that WDR5, ASH2L and RBBP5 form a subcomplex in the absence of MLL (Fig. 1) (Dou et al., 2006; Steward et al., 2006; Thompson et al., 2008; Thompson BA, 2008). Yates et al. (2010) demonstrated a direct interaction of CHD8 with each component of the WAR complex that seem to function as a negative regulator of $H O X A 2$ expression. 

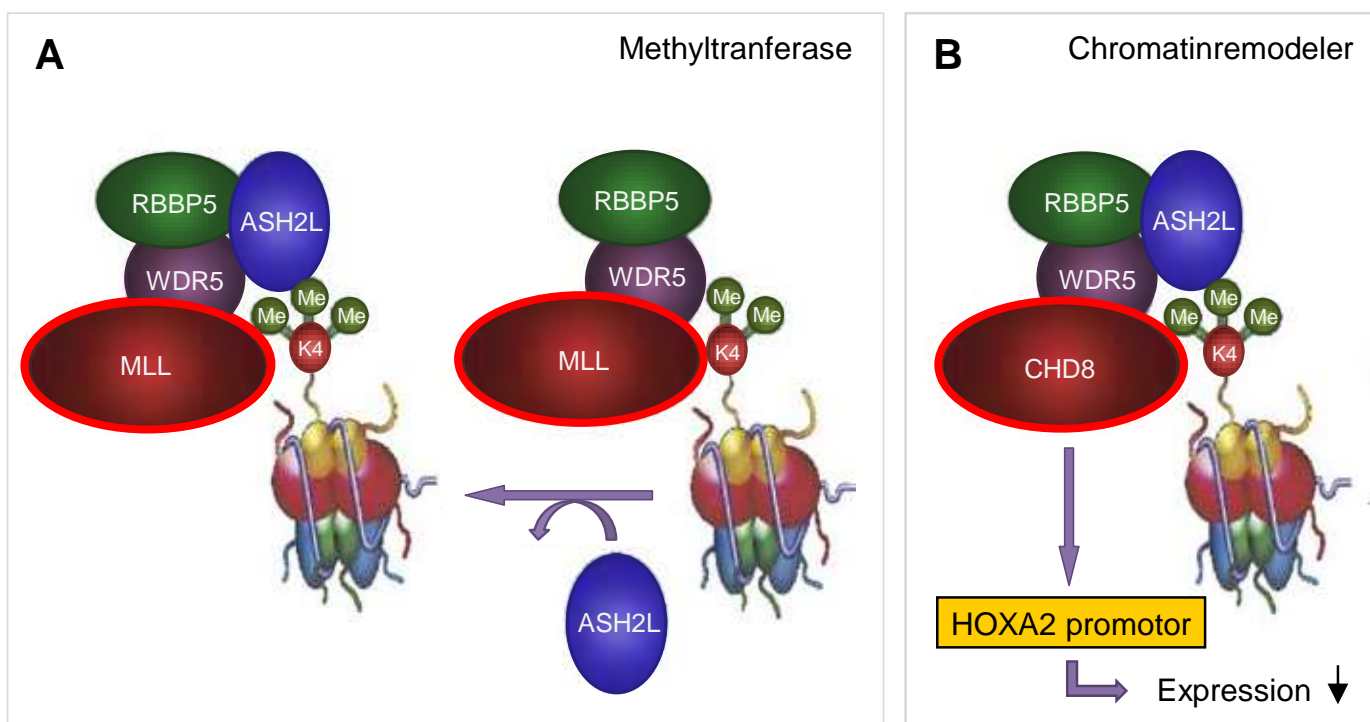

Figure 1: Possible composition of the MLL methyltransferase complex.

(A). MLL is associated via WDR5 with the WAR complex (WDR5, ASH2L and RBBP5). ASH2L is required for trimethylation of H3K4 by the MLL complex. (B) CHD8 seems to bind in the absence of MLL to the WAR complex. This complex might function as a chromatin remodeler which negatively regulates HOXA2 expression. Figure modified after Steward et al. (2006).

Vissers et al. (2004) identified heterozygous mutations within the CHD7 gene to be responsible for a malformation syndrome, called CHARGE syndrome.

\subsection{CHARGE syndrome}

CHARGE syndrome is an autosomal dominant malformation syndrome (OMIM 214800) firstly described in 1979 independently by Hall and Hittner (B. D. Hall, 1979; Hittner et al., 1979). Two years later Pagon et al. (1981) determined the acronym CHARGE association (coloboma, heart defects, atresia choanae, retarded growth and development, genital hypoplasia, ear anomalies/deafness) describing the main features seen in patients and proposed the diagnostic criteria for CHARGE association. Over the years these criteria were refined several times. Blake et al. (1998) defined major (coloboma, atresia of the choanae, cranial nerve disorder and abnormalities of the ear) and minor criteria (heart defects, genital hypoplasia, short stature, developmental delay, tracheoesophageal fistula, orofacial cleft and characteristic facial gestalt) to diagnose CHARGE syndrome. Four major criteria or three major criteria and three out of seven minor criteria must be reported to fulfil the diagnostic criteria of CHARGE syndrome (Blake et al., 1998). An update was defined by Verloes (2005). By identifying CHD7 mutations as the major cause the 
term "CHARGE association" was changed into "CHARGE syndrome" (Vissers et al., 2004; Bergman et al., 2011). Due to the spectrum of malformations, it was hypothesised long time ago that CHARGE syndrome belongs to the neurocristopathies (Siebert et al., 1985; Wright et al., 1986).

\subsection{Neural crest cells - the explorer of the vertebral embryo}

Neural crest cells (NCCs) are a migratory, multipotent cell population which delaminates from the neural fold or dorsal neural tube (Fig. 2) (Gammill and Bronner-Fraser, 2003). NCCs migrate along specific pathways to populate different niches of the embryo (Rickmann et al., 1985; Kontges and Lumsden, 1996; MorinKensicki and Eisen, 1997). Therefore, they are also called the explorers of the vertebral embryo (Gammill and Bronner-Fraser, 2003; Kuriyama and Mayor, 2008).

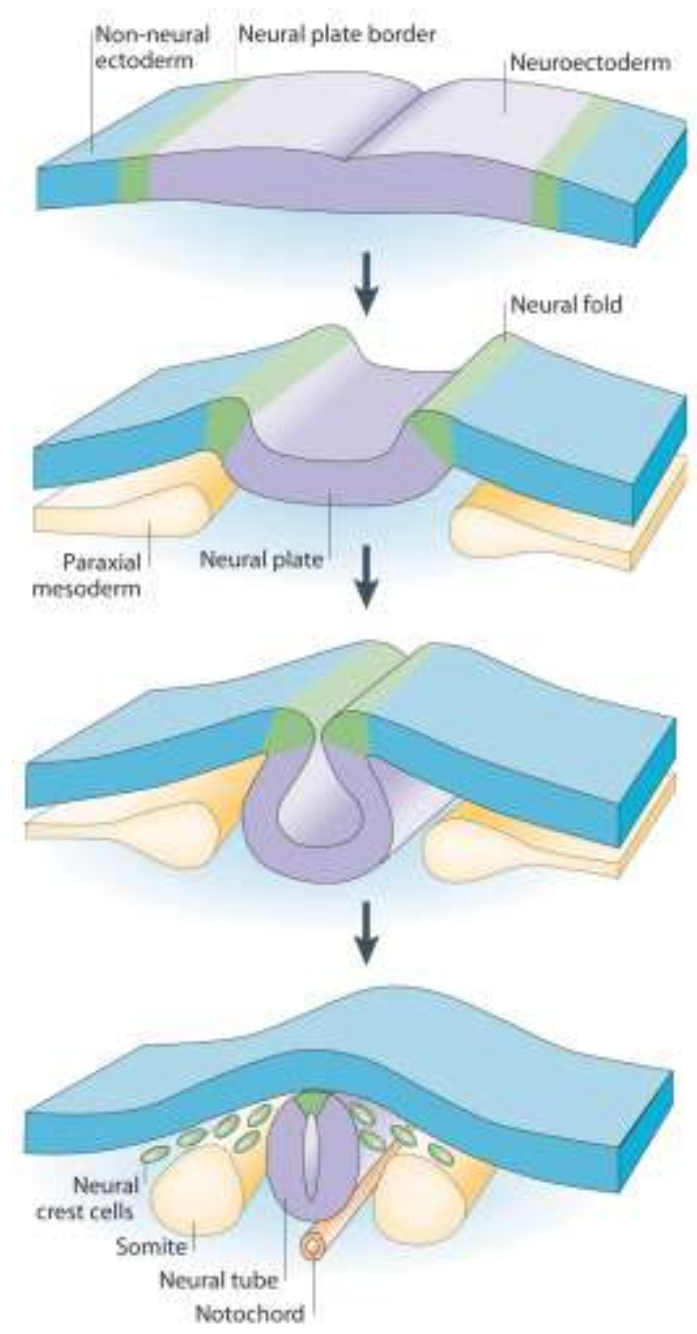

Figure 2: Schematic overview of induction and delamination of neural crest cells. 
The neural plate border (green) separates the neuroectoderm (purple) from the ectoderm (blue) and is specified by signalling from these two tissues and the underlying mesoderm (yellow). During neurulation the neuroectoderm differentiates into the neural plate due to the elevating neural plate border building neural folds. Further elevation of the neural folds forms the neural tube. The dorsal region of the neural tube where the neural folds joined is now referred to as the neural crest. Neural crest cells delaminate from the dorsal region of the neural tube. Figure taken from Gammill and Bronner-Fraser (2003).

Depending on the localisation within the embryo NCCs give rise to different cell types like neurons, muscle, cartilage or pigment cells (Fig. 3). Therefore, they contribute to the formation of many tissues and organs like heart, skin or the peripheral nervous system (Kuriyama and Mayor, 2008). NCCs are divided into four major groups depending on the region of the longitudinal axis of the embryo where the NCCs delaminate: cranial (cephalic) neural crest, trunk neural crest, vagal and sacral neural crest and cardiac neural crest (Gilbert, 2000).

Cranial NCCs differentiate into different sections of the face like jaw and bones of the middle ear, connective tissue, cartilage, odontoblasts and glia cells (Gilbert, 2000). Depending on the migratory pathway trunk NCCs form melanocytes, dorsal root ganglia, adrenal medulla and sympathetic ganglia (Gilbert, 2000). Vagal and sacral NCCs differentiate into the parasympathic nerves of the gut which allow its peristaltic movements (Gilbert, 2000). The cardiac NCCs form the entire musculoconnective tissue wall of the cardiac outflow tract and contribute to the septum that separates the pulmonary circulation from the aorta (Gilbert, 2000; Kirby and Hutson, 2010). Furthermore, this type of NCCs differentiates into cartilage, connective tissue, neurons and melanocytes (Gilbert, 2000; Kirby and Hutson, 2010). 


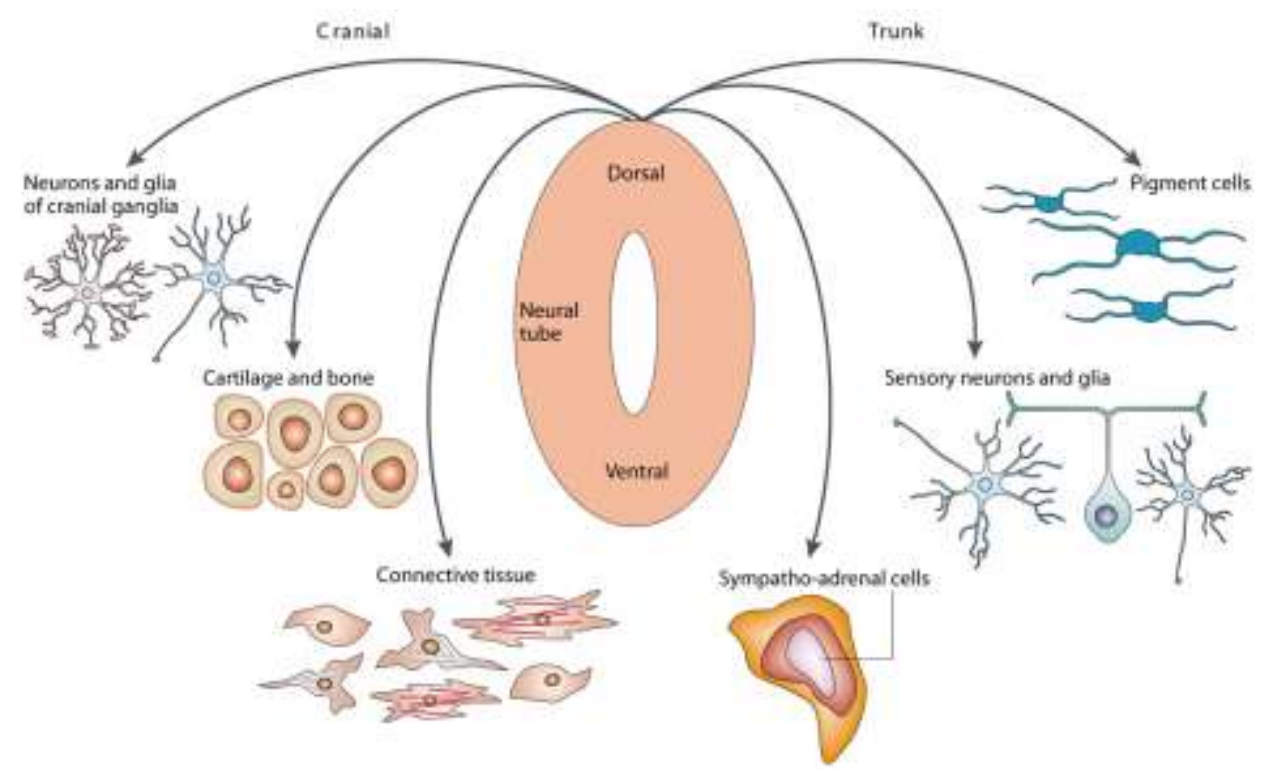

Figure 3: Differentiation of neural crest cells.

Neural crest cells (NCCs) delaminate from the dorsal region of the neural tube and differentiate into multiple cell types. Depending on the region along the longitudinal axis of the embryo different types of NCCs are distinguished which differentiate into different cell types. Cranial NCCs contribute to derivatives of the head region, for instance, neurons and glia of cranial ganglia, cartilage, bones and connective tissue. Trunk NCCs differentiate into melanocytes, sensory neurons and sympathetic ganglia or the adrenal medulla. Figure taken from Knecht and Bronner-Fraser (2002).

\subsection{NCC development}

The NCC development is a complex process of different events starting with the induction of NCCs at the border of the neural plate by interactions of different signals from the surrounding tissues (Knecht and Bronner-Fraser, 2002; Gammill and Bronner-Fraser, 2003). One of the characteristics of NCCs is their ability to migrate. A process called epithelial-mesenchymal transition (EMT) takes place that enables NCCs to delaminate from the dorsal most region of the neural tube and to migrate along specific pathways until they reach their destination where they differentiate into their predetermined cell types (Gammill and Bronner-Fraser, 2003). During EMT fundamental changes in cell morphology and cell adhesion occur (Kuriyama and Mayor, 2008). The typical apical-basal polarity found in the epithelium of premigratory NCCs is lost due to replacement of tight junctions by gap junctions, allowing the exchange of molecules and ions from one cell to the other (Lampe and Lau, 2000; Kuriyama and Mayor, 2008). A group of transmembrane proteins, namely cadherins, seems to be involved in the early EMT process (Shoval et al., 2007). 
Kuriyama and Mayor (2008) assumed that a reduction of type I cadherins in combination with an increase in type II cadherins are linked to NCC EMT. Furthermore, they supposed that matrix metalloproteases which digest the extracellular matrix are required for EMT (Kuriyama and Mayor, 2008).

Once EMT is completed NCCs migrate in clusters along specific pathways to their destination (Kuriyama and Mayor, 2008; Kirby and Hutson, 2010). It has been shown that migrating NCCs are guided by different factors via ligand-receptor signalling (Yazdani and Terman, 2006; Kuriyama and Mayor, 2008).

\subsection{NCC guidance and signalling factors}

The guidance of migrating NCCs is a complex mechanism which involves different factors that interact with each other to provide the information to NCCs so they know where to go. Table 1 shows an overview about some guidance and signalling factors known to play a role during NCC migration.

Table 1: Function of factors involved in early migration and guidance of neural crest cells.

\begin{tabular}{|l|l|}
\hline name & classification and function for NCCs \\
\hline a4ß1 integrin & receptor, controls migration and survival \\
\hline tenascinC & ECM protein, enables delamination \\
\hline WNT1 & growth factor, initiates migration \\
\hline TCF & transcription factor, initiates migration \\
\hline FoxD3 & transcription factor, initiates migration \\
\hline Sox10 & transcription factor, initiates migration \\
\hline semaphorin family & guidance molecule that can attract or repel \\
\hline neurophilin family & receptor for semaphorins \\
\hline plexin family & receptor for semaphorins \\
\hline ephrin family & guidance molecule that can attract or repel \\
\hline Ephrin family & receptor for ephrins \\
\hline Slit family & guidance molecule that can attract or repel \\
\hline Robo family & receptor for Slits \\
\hline HoxA1/B1 & transcription factor, regulates EphA2 expression \\
\hline
\end{tabular}

Table modified after Kirby and Hutson (2010).

Some factors that play a role in the guidance of NCCs should be further described. 


\section{Introduction}

\section{Semaphorins and their receptors neurophilins and plexins}

Some of these guidance factors are members of the semaphorin family which are conserved across divergent animal phyla (Yazdani and Terman, 2006). These transmembrane proteins can attract or repel NCCs. In human and mouse 20 semaphorins exist which can be divided into eight classes (Yazdani and Terman, 2006; Kuriyama and Mayor, 2008). Semaphorins primarily act as ligands (Yazdani and Terman, 2006). All of them possess a conserved sema domain of about 500 amino acids that plays a role during binding to their receptors neurophilins and plexins (Yazdani and Terman, 2006). The members of the transmembrane receptor family neurophilin and plexin are expressed in migrating NCCs. Binding of semaphorins to their receptors leads to alteration of the cytoskeleton by reorganising actin filaments and microtubule (Yu and Kolodkin, 1999). Furthermore, semaphorins and its receptors are involved in the correct migratory patterning of NCCs (Eickholt et al., 1999; Brown et al., 2001; Gitler et al., 2004; Lepore et al., 2006; Sato et al., 2006). It has been shown that Sema4D (sema domain, immunoglobulin domain (Ig), short basic domain, secreted, (semaphorin) 4D) either inhibits or enables cell migration and axon outgrowth depending on the member of the plexin receptor family, Sema4D binds to (Yazdani and Terman, 2006). However, the molecular mechanism behind the function of semaphorins is barely understood.

\section{Ephrins and Eph receptors}

Ephrins serve as ligands for Eph receptors which belong to the subfamily of receptor tyrosine kinases (Kuriyama and Mayor, 2008). Both, ligands and receptors are membrane-bound proteins, so binding of ephrins to their receptors requires direct cell-cell interaction (Kuriyama and Mayor, 2008). Ephrins are involved in the guidance of axons and in cell migration (Egea and Klein, 2007; Rohani et al., 2011). Until now 13 Eph receptors and eight ephrins are known in mammals (Kuriyama and Mayor, 2008). It has been shown in Xenopus laevis that NCCs express Eph receptors while the ligands are expressed in regions of the embryonic tissue where NCCs should either migrate or not migrate (A. Smith et al., 1997). The underlying mechanism by which ephrins mediate their attractive and repulsive effect on NCCs is not clarified. 


\section{Introduction}

\section{Slits and Robo receptors}

The slit/Robo signalling pathway is involved in different processes, such as guidance of axons and trunk NCCs (Kuriyama and Mayor, 2008; Kirby and Hutson, 2010). In mammals three slit ligands and four Robo receptors have been identified until now (Kuriyama and Mayor, 2008). Like semaphorins and ephrins, slits can act bifunctionally having either a stimulating or repulsing effect on migrating NCCs (De Bellard et al., 2003; Kuriyama and Mayor, 2008).

Most of the described factors (semaphorins, ephrins and slits) were shown to act as negative regulators preventing migrating NCCs from invading the region of their expression (Kuriyama and Mayor, 2008). Further factors involved in the process of NCCs guidance exist. However, the whole machinery behind this guidance process of NCCs is hardly understood.

\subsection{Xenopus laevis as a model organism for studying NCC development}

Since NCCs are a feature of vertebrates, they cannot be studied in traditional model organisms like flies, nematodes or yeast. Historically, NCC development has been studied in amphibian embryos like the African clawed frog Xenopus laevis (Collazo et al., 1993). This animal model has the advantage of a relatively fast reproduction cycle and a high number of offspring (Parisis, 2012; Xenbase, n.d.). Furthermore, the breeding can be manipulated by superovulation, so female frogs can lay eggs three to four times a year (Parisis, 2012; Xenbase, n.d.). Amphibian embryos develop externally from the mother animal, so they are easily accessible (Parisis, 2012; Xenbase, n.d.). Oocytes are relatively big with $>1 \mathrm{~mm}$ in diameter and can be manipulated (Parisis, 2012; Xenbase, n.d.). One example is the injection of Morpholinos (MO) into the developing oocyte.

MO are synthetic oligonucleotides consisting of about 25 subunits which comprise of a nucleic acid base bound to a morpholine ring and a non-ionic phosphorodiamidate group linkage (GeneTools, n.d.). MO are used as a tool to knockdown gene functions without degrading their target RNA (GeneTools, n.d.). Blocking the translation or modifying pre-mRNA splicing or inhibiting miRNA is the mechanism by which MO knockdown gene expression (GeneTools, n.d.).

Bajpai et al. (2010) performed MO knockdown experiments with Xenopus laevis embryos for Chd7, a gene that is conserved in human and Xenopus. Downregulation 
of Chd7 revealed features of CHARGE syndrome in Xenopus laevis embryos (Bajpai et al., 2010). Furthermore, Chd7 knockdown experiments demonstrated that the expression of genes involved in early induction of NCCs are not affected while the expression of sox9 (SRY (sex determining region Y)-box 9), twist (basic helix-loophelix transcription factor twist) and slug/snail (Zinc finger protein Slug/ snail family zinc finger) which are involved in NCC specification and early migration processes were downregulated (Bajpai et al., 2010). Therefore, Bajpai et al. (2010) assumed that Chd7 is involved in the regulation of genes which play a role in the formation of migrating NCCs. However, the question in which way Chd7 is involved in the EMT process and in the guidance of migrating NCCs was not answered.

\subsection{The mouse as a model organism and its advantages}

Mouse is a common model organism used for basic research in genetics and human diseases. One of the most striking advantages of the mouse is their similarity to humans in genetics, anatomy and physiology (Simmons, 2008). Over $90 \%$ of the mouse genome resembles the human genome, therefore the mouse afford a good model to study human diseases (Waterston et al., 2002). The mouse genome can be manipulated to induce diseases found in humans either by directly affecting a target gene (knock-out or knock-in) or indirectly by large-scale mutagenesis programmes using chemicals or radiation to induce mutations (Hardouin and Nagy, 2000).

For functional analysis in this work the CHD7 deficient Whirligig mouse line was available that was kindly provided by K. P. Steel (Sanger Centre, Cambridge, United Kingdom) and the Helmholtz Zentrum Munich, Germany. These mice carry a nonsense mutation in exon 11 of the $C h d 7$ gene generated by large-scale ENU $(N$ ethyl- $N$-nitrosourea) mutagenesis programme (Hrabe de Angelis et al., 2000; Hawker et al., 2005). Homozygous mice $\left(C h d 7^{W h i / W h i}\right)$ die at embryonic stage 10.5 (E10.5) whereas heterozygous mice $\left(C h d 7^{W h i /+}\right)$ resemble many features found in CHARGE patients, such as eye and heart defects, ear anomalies, cleft lip and palate and choanal atresia (Bosman et al., 2005). Therefore, the Whirligig mouse line provides a good model to study features of CHARGE syndrome. 


\subsection{Aim of the work}

Chromatin remodelling enzymes exist in large multi-subunit complexes (Schnetz et al., 2010). Are there further CHD7 complex compositions than the ones described in the literature? CHD8 interacts with the WAR complex (Yates et al., 2010). Furthermore, it was demonstrated by our working group that CHD8 interacts with CHD7 (Batsukh et al., 2010). Is CHD7 also associated with the WAR complex? Does CHD7 interact with the members of the WAR complex WDR5, ASH2L and RBBP5? To answer these questions, interaction studies were planned. Coimmunoprecipitation (Co-IP) experiments using HeLa cells should be performed. If CHD7 interacts with components of the WAR complex, where in the cell do the interactions take place? Therefore, Duolink proximity ligation assays (PLA) should be performed. Are the identified interactions direct ones? To clarify this question, direct yeast two-hybrid ( $\mathrm{Y} 2 \mathrm{H})$ experiments were planned.

Little is known about the pathogenesis behind CHARGE syndrome. What kinds of genes are regulated by $\mathrm{CHD} 7$ and what happens if $\mathrm{CHD} 7$ is mutated? To analyse the regulatory effect of $\mathrm{CHD} 7$ on other genes, a genome-wide microarray analysis should be performed on whole embryos of the Whirligig mouse line. The expression profile of heterozygous $\left(C h d 7^{W h i /+}\right)$ and homozygous $\left(C h d 7^{W h i / W h i}\right)$ animals should be compared to the expression profile of wild-type $\left(C h d 7^{+/+}\right)$embryos of the same gender. For the confirmation of the microarray data a RT-qPCR (reverse transcription-quantitative real-time polymerase chain reaction) was planned to perform on some of the identified genes. Is there a gender specific effect on the expression of these genes? To validate this question, the RT-qPCR should be repeated for the same genes using RNA of embryos of the Whirligig mouse line having the opposite gender than the embryos analysed by the microarray.

Depending on the dataset revealed by the genome-wide microarray analysis, further experiments for certain candidate genes could be performed. An option was the functional analysis of candidate genes in different cell lines, in the mouse model or other animal models, such as Xenopus laevis. If required, DNA (deoxyribonucleic acid) samples from CHARGE syndrome patient were available.

Altogether, the aim of this work was to find genes which are regulated by CHD7 and play a role in CHARGE syndrome. Another aim was to identify further interacting partners of CHD7 to help to elucidate the regulatory processes of CHD7. This work 
Introduction

should contribute to better understand the mechanisms behind the pathogenesis of CHARGE syndrome. 


\section{Materials and methods}

\subsection{Materials}

This section includes all material used to conduct the experiments of this work with further category subdivisions.

\subsubsection{Instruments}

All instruments used to perform the experiments in this work are listed in table 2.

Table 2: Instruments

\begin{tabular}{|c|c|}
\hline device name & company \\
\hline $\begin{array}{l}\text { ABI PRISM }{ }^{\circledR} \text { 7900HT Sequence Detection } \\
\text { System }\end{array}$ & Applied Biosystems, Darmstadt \\
\hline advanced primus 96 (PCR cycler) & Peqlab, Erlangen \\
\hline autoclave & Webeco, Fridolfing \\
\hline Basic pH Meter PB-11 & Sartorius, Goettingen \\
\hline Synergy ${ }^{\mathrm{TM}} \mathrm{Mx}$ & BioTek, Bad Friedrichshall \\
\hline camera (agarose gel documentation) & Vilbert Lourmat, Eberhardzell \\
\hline $\begin{array}{l}\text { centrifuges: } \\
4 \mathrm{~K} 15 \\
1-15 \mathrm{~K} \\
\text { Centrifuge } 5415 \mathrm{C} \\
\text { MicroCentrifuge }\end{array}$ & $\begin{array}{l}\text { Sigma, Hamburg } \\
\text { Sigma, Hamburg } \\
\text { Eppendorf, Hamburg } \\
\text { Roth, Karlsruhe }\end{array}$ \\
\hline confocal laser scanning microscope & Olympus, Hamburg \\
\hline drying oven & Memmert, Schwabach \\
\hline electrophorese chamber & Owl Scientific \\
\hline film processor & AGFA, Cologne \\
\hline fluorescence microscope BX60 & Olympus, Munich \\
\hline FluorChem ${ }^{\circledR} \mathrm{Q}$ & Alpha Innotech \\
\hline forceps HWC 110-10 & Hammacher, Solingen \\
\hline $\begin{array}{l}\text { freezer: } \\
-20^{\circ} \mathrm{C} \\
-80^{\circ} \mathrm{C}\end{array}$ & $\begin{array}{l}\text { Liebherr, Ochsenhausen } \\
\text { SANYO, Munich }\end{array}$ \\
\hline haemocytometer chamber & Brand, Wertheim \\
\hline heat sealer & Privileg, Stuttgart \\
\hline
\end{tabular}




\begin{tabular}{|c|c|}
\hline device name & company \\
\hline IKA $^{\circledR}$ Vibrax-VXR (Shaker) & IKA, Staufen \\
\hline $\begin{array}{l}\text { incubators: } \\
\text { bacteria } \\
\text { cell culture } \\
\text { yeast }\end{array}$ & $\begin{array}{l}\text { Adolf Kuehner AG } \\
\text { SANYO, Munich } \\
\text { Kranich }\end{array}$ \\
\hline magnetic stirrer & IKA, Staufen \\
\hline micro centrifuge & Roth, Karlsruhe \\
\hline Microlab Star ${ }^{\text {LET }}$ & Hamilton, Hoechst \\
\hline micro scissors & Roth, Karlsruhe \\
\hline microscope & Leitz, Wetzlar \\
\hline microwave type WR800 & Privileg, Stuttgart \\
\hline Multi 60 well tray lid sterile & Sarstedt, Nuembrecht \\
\hline NanoDrop $^{\mathrm{TM}} 8000$ & Thermo Scientific, Schwerte \\
\hline Novex ${ }^{\circledR}$ Mini Cell (western blot chamber) & Invitrogen, Karlsruhe \\
\hline $\begin{array}{l}\text { pipettes: } \\
1000 \mu 1,200 \mu 1100 \mu 1,20 \mu 1,10 \mu l \\
100 \mu 1 \\
8 \text { canal pipette }\end{array}$ & $\begin{array}{l}\text { Gilson Pipetman } \\
\text { Eppendorf, Hamburg } \\
\text { Biohit/Satorius, Goettingen }\end{array}$ \\
\hline pipette assistant, Accu-jet ${ }^{\circledR}$ & Eppendorf, Hamburg \\
\hline $\begin{array}{l}\text { power supply: } \\
\text { Apelex PS } 304 \text { minipac II } \\
\text { Power PAC } 3000\end{array}$ & $\begin{array}{l}\text { Biostep, Jahnsdorf } \\
\text { BIO-RAD, Munich }\end{array}$ \\
\hline $\begin{array}{l}\text { printer: (gel electrophorese) } \\
\text { P91 } \\
\text { Doc print }{ }^{\mathrm{TM}} \text { VX2 }\end{array}$ & $\begin{array}{l}\text { Mitsubishi } \\
\text { Peqlab, Erlangen }\end{array}$ \\
\hline pump (Multifix Typ MC 1000 PEC) & Kranich GmbH \\
\hline rocking shaker & Heidolph, Schwabach \\
\hline rotating mixer & Karl Hecht, Sondheim / Rhoen \\
\hline rotator SB2 & Stuart, Staffordshire (UK) \\
\hline scale CP3202 S & Sartorius, Goettingen \\
\hline $\begin{array}{l}\text { scissors: } \\
\text { HSB 006-10 } \\
\text { HSB 390-10 }\end{array}$ & $\begin{array}{l}\text { Hammacher, Solingen } \\
\text { Hammacher, Solingen }\end{array}$ \\
\hline spectral photometer & Eppendorf, Hamburg \\
\hline SpeedVac concentrator SVC $100 \mathrm{H}$ & Schuett, Goettingen \\
\hline
\end{tabular}


Materials and methods

\begin{tabular}{l|l}
\hline device name & company \\
\hline steriliser & Memmert, Schwabach \\
\hline sterile laboratory hood & HERAsafe, Schwerte \\
\hline tank blot & Bio-Rad, Munich \\
\hline thermomixer 5436 & Eppendorf, Hamburg \\
\hline TissueLyser TL & Qiagen, Hilden \\
\hline Turboblotter ${ }^{\text {TM }}$ & Schleicher \& Schuell, Dassel \\
\hline UV table ECX-F20-M & PeqLab, Erlangen \\
\hline Vortex-Genie ${ }^{\circledR} 2$ & Scientific industries \\
\hline waterbath & Koettermann, Uetze/Haenigsen \\
\hline X-ray cassette & Rego X-ray, Augsburg \\
\hline 7900HT Fast Real-Time PCR-System & Applied Biosystems, Darmstadt \\
\hline $3500 x$ genetic Analyzer & Applied Biosystems, Darmstadt \\
\hline 2720 Thermalcycler & Applied Biosystems, Darmstadt
\end{tabular}

\subsubsection{Consumable materials}

Table 3 gives an overview about the consumable materials and the manufacturing companies.

Table 3: Consumable materials

\begin{tabular}{l|l}
\hline product name & company \\
\hline ABgene Diamond Ultra 384 well PCR plate & Thermo Scientific, Schwerte \\
\hline $\begin{array}{l}\text { cell culture flasks: } \\
25 \mathrm{~cm}^{2} \text { with filter } \\
75 \mathrm{~cm}^{2} \text { with filter } \\
75 \mathrm{~cm}^{2} \text { without filter }\end{array}$ & $\begin{array}{l}\text { Sarstedt, Nuembrecht } \\
\text { Sarstedt, Nuembrecht } \\
\text { CellSTAR, Darmstadt }\end{array}$ \\
\hline CL-XPosure ${ }^{\text {TM }}$ Film $($ Clear blue X-Ray film) & Thermo Scientific, Schwerte \\
\hline cover slides, $24 \times 60 \mathrm{~mm}($ Menzel-Glaeser $)$ & Thermo Scientific, Schwerte \\
\hline Cryotube & $\begin{array}{l}\text { Greiner Bio-one, } \\
\text { Frickenhausen }\end{array}$ \\
\hline cuvette & Sarstedt, Nuembrecht \\
\hline E-Cups $(2 \mathrm{ml}, 1.5 \mathrm{ml}, 750 \mu \mathrm{l})$ & Sarstedt, Nuembrecht \\
\hline Falcon tubes $(15 \mathrm{ml}, 50 \mathrm{ml})$ & CellSTAR, Darmstadt \\
\hline
\end{tabular}




\section{product name}

Filter tips $(1000 \mu 1,200 \mu 1,100 \mu 1,20 \mu 1,10 \mu 1)$

Folded Filters

Glass pipettes

Hybond $^{\mathrm{TM}}$ CExtra, Nitrocellulose $0.45 \mu \mathrm{m}$

Hybond $^{\mathrm{TM}} \mathrm{P}$, PVDF Transfer Membran $0.45 \mu \mathrm{m}$

Micro Screwtube $1.5 \mathrm{ml}$

MultiScreen ${ }^{\circledR}$ Filter Plates

NuPAGE $^{\circledR} 4-12 \%$ Bis-Tris Gel

NuPAGE $^{\circledR} 3-8 \%$ Tris-Acetate Gel

PCR-Cups

Petri dish (big)

Petri dish (small)

Pipette tips

QPCR Adhesive Clear Seals

scalpel

Serological pipettes $(10 \mathrm{ml}, 5 \mathrm{ml}, 2 \mathrm{ml})$

Serological pipettes $(25 \mathrm{ml})$

sterile surgical blades

Superfrost $^{\circledR}$ Plus (Menzel-Glaeser)

Transfection tube

Transfer pipettes $3.5 \mathrm{ml}$

Whatman Paper

6-well plate

24 well plate

96-well plate (qPCR)

96-well plate (Bradford)

2-well, 4-well and 8-well slide (BD Falcon ${ }^{\mathrm{TM}}$ culture slide) company

Kisker, Steinfurt

Satorius, Goettingen

Schuett, Goettingen

Amersham, Freiburg

Amersham, Freiburg

Sarstedt, Nuembrecht

Merck Millipore, Darmstadt

Invitrogen, Karlsruhe

Invitrogen, Karlsruhe

Invitrogen, Karlsruhe

Greiner Bio-one,

Frickenhausen

Thermo Scientific, Schwerte

Eppendorf, Hamburg

4titude, Berlin

Pfm medical, Cologne

Sarstedt, Nuembrecht

Greiner Bio-one, Frickenhausen

Braun, Tuttlingen

Thermo Scientific, Schwerte

Sarstedt, Nuembrecht

Sarstedt, Nuembrecht

Schleicher and Schuell

Sarstedt, Nuembrecht

Sarstedt, Nuembrecht

4titude, Berlin

Thermo Scientific, Schwerte

BD Bioscines, Erembodegem (Belgium) 


\subsubsection{Kits}

The Kits used in this work are listed in table 4.

Table 4: Kits

\begin{tabular}{|c|c|}
\hline product name & company \\
\hline BigDye $^{\circledR}$ & Life Technologies, Darmstadt \\
\hline Duolink In Situ Detection Reagents Orange & Olink, Uppsala (Sweden) \\
\hline Duolink In Situ PLA probe anti-Goat PLUS & Olink, Uppsala (Sweden) \\
\hline Duolink In Situ PLA probe anti-Rabbit MINUS & Olink, Uppsala (Sweden) \\
\hline $\begin{array}{l}\text { DYEnamic ET Terminator Cycle Sequencing Kit } \\
\text { (ET-Mix) }\end{array}$ & GE Healthcare, Munich \\
\hline Immunoprecipitation Kit (Protein G) & Roche, Penzberg \\
\hline In-Fusion ${ }^{\mathrm{TM}}$ Advantage PCR Cloning Kit & Clontech, Heidelberg \\
\hline Mini Plasmid Kit & Qiagen, Hilden \\
\hline $\begin{array}{l}\text { mMESSAGE mMACHINE }{ }^{\circledR} \text { SP6 Transcription } \\
\text { Kit }\end{array}$ & Life Technologies, Darmstadt \\
\hline $\begin{array}{l}\text { mMESSAGE mMACHINE }{ }^{\circledR} \text { T7 Transcription } \\
\text { Kit }\end{array}$ & Life Technologies, Darmstadt \\
\hline MSB $^{\circledR}$ Spin PCRapace & Invitek, Berlin \\
\hline $\begin{array}{l}\text { NE-PER Nuclear and Cytoplasmic Extraction } \\
\text { Reagents }\end{array}$ & Thermo Scientific, Schwerte \\
\hline Novocastra ${ }^{\mathrm{TM}}$ Novostain Universal Detection Kit & Leica Biosystems, Wetzlar \\
\hline $\begin{array}{l}\text { Platinum }{ }^{\circledR} \text { SYBR }^{\circledR} \text { Green qPCRSuperMix-UDG } \\
\text { with ROX }\end{array}$ & Invitrogen, Karlsruhe \\
\hline Pure Link ${ }^{\circledR}$ HiPure Plasmid (Midiprep Kit) & Invitrogen, Karlsruhe \\
\hline QIAquick Gel Extraction Kit & Qiagen, Hilden \\
\hline RNAspin Mini & GE Healthcare, Munich \\
\hline RNeasy $^{\circledR}$ mini Kit (250) & Qiagen, Hilden \\
\hline $\begin{array}{l}\text { SuperSignal } \\
\text { Substrate }\end{array}$ & Thermo Scientific, Schwerte \\
\hline Xfect $^{\mathrm{TM}}$ & Clontech, Heidelberg \\
\hline
\end{tabular}

\subsubsection{Ready to use buffers and mediums}

Table 5 includes buffers and mediums which were ready to use as well as the companies selling these products. 
Materials and methods

Table 5: Ready to use buffers and mediums

\begin{tabular}{|c|c|}
\hline product name & company \\
\hline Aqua-Poly/Mount Coverslipping Medium & Polysciences, Eppelheim \\
\hline BSA $(100 x)$ & BioLabs, Frankfurt am Main \\
\hline Developer G153 A+B & AGFA, Cologne \\
\hline 3,3'-Diaminobenzidine (DAB) & Roche, Penzberg \\
\hline Direct lysis buffer & Peqlab, Erlangen \\
\hline Dulbecco’s Modified Eagle Medium (DMEM) & PAN Biotech, Nuremberg \\
\hline Dulbecco's Phosphate-Buffered Saline (DPBS) & PAN Biotech, Nuremberg \\
\hline Fetal bovine serum & PAN Biotech, Nuremberg \\
\hline MEM NEAA & $\begin{array}{l}\text { Gibco, (Life Technologies), } \\
\text { Darmstadt }\end{array}$ \\
\hline NuPAGE ${ }^{\circledR}$ LDS Sample Buffer (4 x) & Invitrogen, Karlsruhe \\
\hline NuPAGE $^{\circledR}$ MES SDS Running Buffer $(20 \mathrm{x})$ & Invitrogen, Karlsruhe \\
\hline NuPAGE $^{\circledR}$ MOPS SDS Running Buffer (20 x) & Invitrogen, Karlsruhe \\
\hline NuPAGE $^{\circledR}$ Tris-Acetate SDS Running Buffer $(20 \mathrm{x})$ & Invitrogen, Karlsruhe \\
\hline NuPAGE $^{\circledR}$ Transfer Buffer $(20 \mathrm{x})$ & Invitrogen, Karlsruhe \\
\hline OPTI-MEM $^{\circledR}$ I + GlutaMax - I & Invitrogen, Karlsruhe \\
\hline P1 Resuspension buffer & Qiagen, Hilden \\
\hline P2 Lysis Puffer & Qiagen, Hilden \\
\hline P3 Neutralisation buffer & Qiagen, Hilden \\
\hline Rapidfixer & AGFA, Cologne \\
\hline S.O.C. Medium & Invitrogen, Karlsruhe \\
\hline TE-Buffer & Invitrogen, Karlsruhe \\
\hline TrypLE $^{\mathrm{TM}}$ Express & Invitrogen, Karlsruhe \\
\hline
\end{tabular}

\subsubsection{Chemicals}

In table 6 all chemicals which were used for the experiments in this work and the corresponding companies selling these products are listed. 
Table 6: Chemicals

\begin{tabular}{|c|c|}
\hline product name & company \\
\hline Acetic acid & Merck, Darmstadt \\
\hline Acetic Anhydrite & Sigma, Hamburg \\
\hline Acetone & Merck, Darmstadt \\
\hline Agar-Agar, Kobe I & Roth, Karlsruhe \\
\hline Agarose & Bio-Budget, Krefeld \\
\hline Albumin Fraction V (bovine serum albumin (BSA)) & Roth, Karlsruhe \\
\hline Ampicillin & Roth, Karlsruhe \\
\hline Ampuwa & Fresenius, Bad Homburg \\
\hline Aprotinin & Sigma-Aldrich, Deisenhofen \\
\hline$\beta$-Mercaptoethanol & Sigma-Aldrich, Deisenhofen \\
\hline Boehringer Mannheim Blocking Reagent (BMB) & Roche, Penzberg \\
\hline 5-Brom-4-chlor-3-indolylphosphate (BCIP) & Roche, Penzberg \\
\hline Bromphenolblue & Roth, Karlsruhe \\
\hline Calcium chloride $\left(\mathrm{CaCl}_{2}\right)$ & Roth, Karlsruhe \\
\hline $\begin{array}{l}\text { 3-[(3-Cholamidopropyl)dimethylammonio]-1- } \\
\text { propanesulfonate (CHAPS) }\end{array}$ & Sigma-Aldrich, Deisenhofen \\
\hline Chloroform & J.T. Baker, Griesheim \\
\hline Coomassie ${ }^{\circledR}$ Brilliant Blue R250 Powder & $\begin{array}{l}\text { SERVA Electrophoresis, } \\
\text { Heidelberg }\end{array}$ \\
\hline $\begin{array}{l}\text { Desoxy-Nucleotid-Triphosphate (dNTPs) } \\
\mathrm{mM})\end{array}$ & Invitrogen, Karlsruhe \\
\hline Diethylpyrocarbonat (DEPC) & Roth, Karlsruhe \\
\hline Dimethyl sulfoxid (DMSO) & Merck, Darmstadt \\
\hline Disodium phosphate $\left(\mathrm{Na}_{2} \mathrm{HPO}_{4}\right)$ & Roth, Karlsruhe \\
\hline 1,4-Dithiothreitol (DTT) & Invitrogen, Karlsruhe \\
\hline DO Supplement (-Ade/-His/-Leu/-Trp) & Clontech, Heidelberg \\
\hline Ethanol & J.T. Baker, Griesheim \\
\hline Ethidium bromide & AppliChem, Darmstadt \\
\hline Ethylene diamine tetraacetic acid (EDTA) & AppliChem, Darmstadt \\
\hline Ethylene glycol tetraacetic acid (EGTA) & AppliChem, Darmstadt \\
\hline
\end{tabular}


Materials and methods

product name

Ficoll 400

FITC Phalloidin

Formaldehyde

Formamid

Glycerol

Glycin

G-Protein

Guanidin hydrochloride

Heparin

Hepes

Hydrochloric acid $(\mathrm{HCl})$

Hydrogen peroxide

2-(4-(2-Hydroxyethyl)-1-piperazinyl)-ethansulfon acid (Hepes)

Isopropanol

Leupeptin

Lipofectamine 2000 Reagent

Magnesium chloride $\left(\mathrm{MgCl}_{2}\right)$

Magnesium sulphate $\left(\mathrm{MgSO}_{4}\right)$

Maleic acid

Methanol

Milkpowder

Monosodium phosphate $\left(\mathrm{NaH}_{2} \mathrm{PO}_{4}\right)$

Nitro blue tetrazolium (NBT)

Mineral oil

Nitrogen (liquid)

Paraffin

Paraformaldehyde

Pepstatin company

Sigma-Aldrich, Deisenhofen

Sigma-Aldrich, Deisenhofen

Invitrogen, Karlsruhe

Roth, Karlsruhe

Roth, Karlsruhe

Roth, Karlsruhe

Roche, Penzberg

Serva, Heidelberg

Roth, Karlsruhe

AppliChem, Darmstadt

Merck, Darmstadt

Roth, Karlsruhe

Sigma, Hamburg

J.T. Baker, Griesheim

Sigma, Hamburg

Invitrogen, Karlsruhe

Bioline, Luckenwalde

Roth, Karlsruhe

AppliChem, Darmstadt

Roth, Karlsruhe

Roth, Karlsruhe

Roth, Karlsruhe

Roche, Penzberg

Sigma, Hamburg

Air Liquide, Kornwestheim

Roth, Karlsruhe

Roth, Karlsruhe

Sigma, Hamburg 


\begin{tabular}{|c|c|}
\hline product name & company \\
\hline Peptone & Roth, Karlsruhe \\
\hline Phenol & Biomol, Hamburg \\
\hline Phenylmethanesulfonylfluoride (PMSF) & Sigma, Hamburg \\
\hline Polyvinylpyrollidone & Sigma, Hamburg \\
\hline Ponceau S & Sigma, Hamburg \\
\hline Potassium chloride $(\mathrm{KCl})$ & Roth, Karlsruhe \\
\hline Potassium ferricyanide $\left(\mathrm{K}_{3} \mathrm{Fe}(\mathrm{CN})_{6}\right)$ & Sigma, Hamburg \\
\hline Potassium ferrocyanide $\left(\mathrm{K}_{4} \mathrm{Fe}(\mathrm{CN})_{6}\right)$ & Sigma, Hamburg \\
\hline Potassium nitrate $\left(\mathrm{KNO}_{3}\right)$ & Merck, Darmstadt, \\
\hline Proteinase $\mathrm{K}$ & Roche, Penzberg \\
\hline Roti-Nanoquant & Roth, Karlsruhe \\
\hline Roti Safe & Roth, Karlsruhe \\
\hline Salmon sperm DNA & Sigma, Hamburg \\
\hline Sodium azide & Roth, Karlsruhe \\
\hline Sodium bicarbonate $\left(\mathrm{NaHCO}_{3}\right)$ & Roth, Karlsruhe \\
\hline Sodium chloride $(\mathrm{NaCl})$ & AppliChem, Darmstadt \\
\hline Sodium citrate & Roth, Karlsruhe \\
\hline Sodium dodecyl sulfate (SDS) & Serva, Heidelberg \\
\hline Sodium hydroxide solution $(\mathrm{NaOH})$ & Merck, Darmstadt \\
\hline Torula RNA & Sigma, Hamburg \\
\hline Triethanolamine & Roth, Karlsruhe \\
\hline Tris(hydroxymethyl)-aminomethan (TRIS) & AppliChem, Darmstadt \\
\hline Triton-X-100 & Serva, Heidelberg \\
\hline TRIZOL Reagent & Invitrogen, Karlsruhe \\
\hline Tween 20 & AppliChem, Darmstadt \\
\hline $\begin{array}{l}\text { Vectashield with } 4 \text { ',6-Diamidin-2-phenylindol } \\
\text { (DAPI) }\end{array}$ & Vector, Burlingame (USA) \\
\hline Yeast extract & Roth, Karlsruhe \\
\hline $\mathrm{X}-\mathrm{Gal}$ & Biomol, Hamburg \\
\hline
\end{tabular}


Materials and methods

\subsubsection{Buffers and solutions}

The following tables show how to prepare the buffers needed for different experiments.

Table 7: Agarose gel electrophorese

\begin{tabular}{lll}
\hline Buffer/solution & Ingredients & \\
\hline Loading Buffer $(\mathbf{5 0} \mathbf{~ m l})$ & $50 \%(\mathrm{v} / \mathrm{v})$ & Glycerol \\
& $0.1 \mathrm{M}$ & EDTA \\
& $0.02 \%(\mathrm{w} / \mathrm{v})$ & Xylencyanol \\
& $0.02 \%(\mathrm{w} / \mathrm{v})$ & Bromphenol Blue \\
& $\underline{0.02 \%(\mathrm{w} / \mathrm{v})}$ & SDS \\
& ad $50 \mathrm{ml} \mathrm{ddH} \mathrm{H}_{2} \mathrm{O}$ & \\
\hline $\mathbf{1} \mathbf{x}$ TAE & $40 \mathrm{mM}$ & Tris-Acetate $(\mathrm{pH} 8.5)$ \\
& $2 \mathrm{mM}$ & $0.5 \mathrm{M}$ EDTA \\
\hline $\mathbf{5} \mathbf{x}$ TBE & $450 \mathrm{mM}$ & Tris base \\
& $450 \mathrm{mM}$ & boric acid \\
& $20 \mathrm{mM}$ & EDTA \\
& adjust pH to 8 &
\end{tabular}


Materials and methods

Table 8: Co-immunoprecipitation (Co-IP)

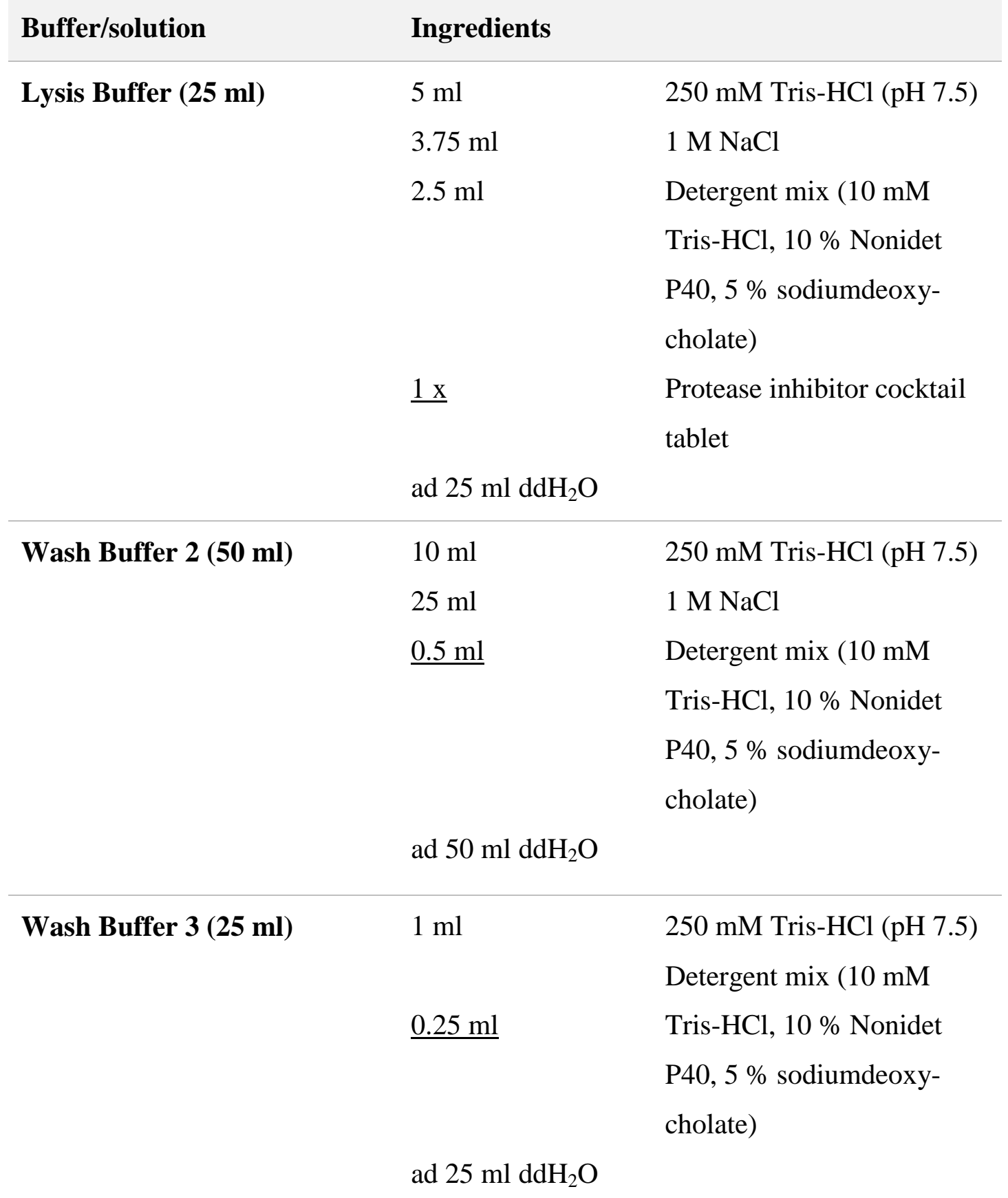

Table 9: DAB staining

\section{Buffer/solution Ingredients}

Citrate-EDTA Buffer $(\mathbf{1}$ L) $\quad 2.1 \mathrm{~g} \quad$ Citric Acid

$0.74 \mathrm{~g} \quad$ EDTA

adjust $\mathrm{pH}$ to 6.2

$\underline{500 \mu 1} \quad$ Tween 20

ad $1 \mathrm{LddH}_{2} \mathrm{O}$ 
Table 10: Duolink

\begin{tabular}{|c|c|c|}
\hline Buffer/solution & Ingredients & \\
\hline \multirow[t]{7}{*}{ Wash buffer A (1 L) } & $8.8 \mathrm{~g}$ & $\mathrm{NaCl}$ \\
\hline & $1.2 \mathrm{~g}$ & Tris base \\
\hline & $\underline{500 \mu 1}$ & Tween 20 \\
\hline & ad $0.8 \mathrm{~L} \mathrm{ddH}_{2} \mathrm{O}$ & \\
\hline & adjust $\mathrm{pH}$ to 7.4 & \\
\hline & ad $1 \mathrm{LddH}_{2} \mathrm{O}$ & \\
\hline & filter $(0.22 \mu \mathrm{m})$ & \\
\hline \multirow[t]{6}{*}{ Wash buffer B (1 L) } & $5.84 \mathrm{~g}$ & $\mathrm{NaCl}$ \\
\hline & $\underline{24.228 \mathrm{~g}}$ & Tris \\
\hline & ad $0.5 \mathrm{~L} \mathrm{ddH}_{2} \mathrm{O}$ & \\
\hline & adjust $\mathrm{pH}$ to 7.5 & \\
\hline & ad $1 \mathrm{~L} \mathrm{ddH}_{2} \mathrm{O}$ & \\
\hline & filter $(0.22 \mu \mathrm{m})$ & \\
\hline
\end{tabular}

Table 11: Polymerase chain reaction (PCR)

\begin{tabular}{lll}
\hline Buffer/solution & Ingredients & \\
\hline dNTPs & $10 \mu 1$ & dATP \\
& $10 \mu 1$ & dCTP \\
$10 \mu l$ & dGTP \\
$10 \mu 1$ & dTTP
\end{tabular}

Table 12: RNA isolation

\begin{tabular}{|lll|}
\hline Buffer/solution & Ingredients & \\
\hline DEPC & $\underline{1 \mathrm{ml}}$ & Diethylpyrocarbonate \\
& ad $1 \mathrm{~L} \mathrm{ddH}_{2} \mathrm{O}$ & \\
\hline $\mathbf{7 5} \%$ Ethanol/DEPC & $75 \%$ & Ethanol \\
& $25 \%$ & DEPC \\
\hline
\end{tabular}


Materials and methods

Table 13: Western blots

\begin{tabular}{|c|c|c|}
\hline Buffer/solution & Ingredients & \\
\hline Antibody Diluent & $\begin{array}{l}2 \% \\
\text { in TBST }\end{array}$ & BSA or milkpouder \\
\hline Blocking Buffer & $\begin{array}{l}5 \% \\
\text { in TBST }\end{array}$ & BSA or milkpouder \\
\hline $\begin{array}{l}\text { Stripping Buffer (1 L, pH } \\
\text { 2.2) }\end{array}$ & $\begin{array}{l}1 \mathrm{~g} \\
15 \mathrm{~g} \\
\underline{10 \mathrm{ml}} \\
\mathrm{ad} 1 \mathrm{~L} \mathrm{ddH} \mathrm{H}_{2} \mathrm{O}\end{array}$ & $\begin{array}{l}\text { SDS } \\
\text { Glycin } \\
\text { Tween } 20\end{array}$ \\
\hline TBST (1 L) & $\begin{array}{l}10 \mathrm{ml} \\
9 \mathrm{~g} \\
\frac{1 \mathrm{ml}}{\operatorname{ad} 1 \mathrm{~L} \mathrm{ddH}_{2} \mathrm{O}}\end{array}$ & $\begin{array}{l}\text { Tris-HCl (1 M) } \\
\mathrm{NaCl} \\
\text { Tween } 20\end{array}$ \\
\hline $\begin{array}{l}1 \text { x Transfer Buffer }(100 \text { ml) } \\
\text { (semidry) }\end{array}$ & 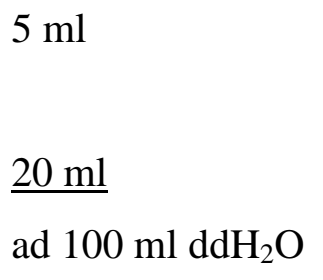 & $\begin{array}{l}\text { NuPAGE Transfer Buffer } \\
(20 \mathrm{x}) \\
\text { Methanol }\end{array}$ \\
\hline $\begin{array}{l}1 \text { x Transfer Buffer }(\mathbf{1} \text { L) } \\
\text { (Wet Blot) }\end{array}$ & $\begin{array}{l}6.05 \mathrm{~g} \\
28.52 \mathrm{~g} \\
0.1 \% \\
\frac{20 \%}{\mathrm{ad} 1 \mathrm{~L} \mathrm{ddH} \mathrm{H}_{2} \mathrm{O}}\end{array}$ & $\begin{array}{l}\text { Tris } \\
\text { Glycin } \\
\text { SDS } \\
\text { Methanol }\end{array}$ \\
\hline Wash Buffer & $\begin{array}{l}2 \% \\
\text { in TBST }\end{array}$ & milkpouder \\
\hline
\end{tabular}


Materials and methods

Table 14: Whole mount in situ hybridisation

\begin{tabular}{|c|c|c|}
\hline Buffer/solution & Ingredients & \\
\hline \multirow[t]{6}{*}{ APB $(100 \mathrm{ml})$} & $10 \mathrm{ml}$ & Tris $(1 \mathrm{M})$ \\
\hline & $5 \mathrm{ml}$ & $\mathrm{MgCl}_{2}(1 \mathrm{M})$ \\
\hline & $2 \mathrm{ml}$ & $\mathrm{NaCl}(5 \mathrm{M})$ \\
\hline & $500 \mu 1$ & Tween $20(20 \%)$ \\
\hline & adjust $\mathrm{pH} 9$ & \\
\hline & 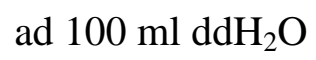 & \\
\hline Boehringer Mannheim & $10 \%$ & $\mathrm{BMB}$ \\
\hline Blocking Reagent (BMB) & ad MAB & \\
\hline \multirow[t]{4}{*}{ Denhardts $(100 \mathrm{x})$} & $1 \mathrm{~g}$ & BSA \\
\hline & $1 \mathrm{~g}$ & Ficoll 400 \\
\hline & $\underline{1 \mathrm{~g}}$ & Polyvinylpyrollidone \\
\hline & ad $50 \mathrm{ml} \mathrm{ddH_{2 }} \mathrm{O}$ & \\
\hline \multirow[t]{9}{*}{ Hybridisation Buffer } & $250 \mathrm{ml}$ & Formamide \\
\hline & $125 \mathrm{ml}$ & $\operatorname{SSC}(20 x)$ \\
\hline & $50 \mathrm{ml}$ & Torula RNA $(10 \mathrm{mg} / \mathrm{ml})$ \\
\hline & $50 \mathrm{mg}$ & Heparin \\
\hline & $5 \mathrm{ml}$ & Denhardts $(100 \mathrm{x})$ \\
\hline & $0.5 \mathrm{ml}$ & Tween 20 \\
\hline & $0.5 \mathrm{~g}$ & CHAPS \\
\hline & $2.5 \mathrm{ml}$ & EDTA (2 M) \\
\hline & 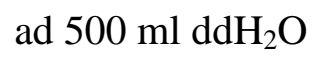 & \\
\hline \multirow[t]{3}{*}{ MAB (5 x) } & $100 \mathrm{mM}$ & Maleic acid \\
\hline & $150 \mathrm{mM}$ & $\mathrm{NaCl}$ \\
\hline & adjust $\mathrm{pH} 7.5$ & \\
\hline
\end{tabular}


Materials and methods

\begin{tabular}{|c|c|c|}
\hline Buffer/solution & Ingredients & \\
\hline \multirow[t]{7}{*}{$\operatorname{MBS}(1 \mathrm{x})$} & $10 \mathrm{mM}$ & Hepes (pH 7) \\
\hline & $88 \mathrm{mM}$ & $\mathrm{NaCl}$ \\
\hline & $1 \mathrm{mM}$ & $\mathrm{KCl}$ \\
\hline & $2.4 \mathrm{mM}$ & $\mathrm{NaHCO}_{3}$ \\
\hline & $0.82 \mathrm{mM}$ & $\mathrm{MgSO}_{4}$ \\
\hline & $0.41 \mathrm{mM}$ & $\mathrm{CaCl}_{2}$ \\
\hline & $0.66 \mathrm{mM}$ & $\mathrm{KNO}_{3}$ \\
\hline \multirow[t]{4}{*}{ MEMFA } & $0.1 \mathrm{M}$ & MOPS \\
\hline & $2 \mathrm{mM}$ & EGTA \\
\hline & $1 \mathrm{mM}$ & $\mathrm{MgSO}_{4}$ \\
\hline & $4 \%$ & Formaldehyde \\
\hline \multirow[t]{4}{*}{$\operatorname{PBS}(10 x)$} & $3.5 \mathrm{M}$ & $\mathrm{NaCl}$ \\
\hline & $37 \mathrm{mM}$ & $\mathrm{NaH}_{2} \mathrm{PO}_{4}$ \\
\hline & $150 \mathrm{mM}$ & $\mathrm{Na}_{2} \mathrm{HPO}_{4}$ \\
\hline & adjust $\mathrm{pH} 7.3$ & \\
\hline \multirow[t]{5}{*}{ Phosphatase Buffer } & $10 \mathrm{ml}$ & Tris-Cl (1 M, pH 9.5) \\
\hline & $5 \mathrm{ml}$ & $\mathrm{MgCl}_{2}(1 \mathrm{M})$ \\
\hline & $2 \mathrm{ml}$ & $\mathrm{NaCl}(5 \mathrm{M})$ \\
\hline & $\underline{0.5 \mathrm{ml}}$ & Tween 20 \\
\hline & ad $100 \mathrm{ml} \mathrm{ddH} \mathrm{H}_{2} \mathrm{O}$ & \\
\hline \multirow[t]{2}{*}{ PTw } & $1 \mathrm{x}$ & PBS \\
\hline & $0.1 \%$ & Tween 20 \\
\hline \multirow[t]{3}{*}{$\operatorname{SSC}(20 x)$} & $0.3 \mathrm{M}$ & Sodium citrate \\
\hline & $3 \mathrm{M}$ & $\mathrm{NaCl}$ \\
\hline & adjust pH 7 & \\
\hline \multirow{5}{*}{$\begin{array}{l}\text { X-Gal } \\
(10 \text { ml })\end{array}$} & $250 \mu 1$ & $\mathrm{X}-\mathrm{Gal}(40 \mathrm{mg} / \mathrm{ml})$ \\
\hline & $200 \mu 1$ & $\mathrm{~K}_{3} \mathrm{Fe}(\mathrm{CN})_{6}$ \\
\hline & $200 \mu 1$ & $\mathrm{~K}_{4} \mathrm{Fe}(\mathrm{CN})_{6}$ \\
\hline & $\underline{20 \mu 1}$ & $\mathrm{MgCl}_{2}(1 \mathrm{M})$ \\
\hline & ad $10 \mathrm{ml}$ PBS (1 x) & \\
\hline
\end{tabular}


Table 15: Yeast two-hybrid

\begin{tabular}{|c|c|c|}
\hline Buffer/solution & Ingredients & \\
\hline \multirow[t]{3}{*}{ Glucose (40 \%) } & $\underline{200 \mathrm{~g}}$ & Glucose \\
\hline & 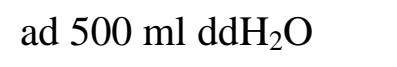 & \\
\hline & autoclave and filtrate & \\
\hline \multirow[t]{3}{*}{ PEG (50 \%) } & $\underline{50 \mathrm{~g}}$ & PEG 4000 \\
\hline & 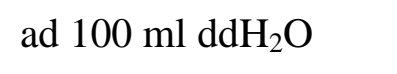 & \\
\hline & autoclave & \\
\hline \multirow[t]{3}{*}{$\operatorname{LiAc}(10 x)$} & $\underline{6.598 \mathrm{~g}}$ & LiAc \\
\hline & 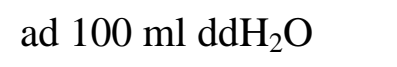 & \\
\hline & autoclave & \\
\hline \multirow[t]{3}{*}{ TE $(10 x)$} & $10 \mathrm{ml}$ & $1 \mathrm{M}$ Tris- $\mathrm{HCl}(\mathrm{pH} 7.4-8)$ \\
\hline & $\underline{2 \mathrm{ml}}$ & 0.5 M EDTA (pH 8) \\
\hline & $\begin{array}{l}\text { ad } 100 \mathrm{ml} \mathrm{ddH}_{2} \mathrm{O} \\
\text { autoclave }\end{array}$ & \\
\hline
\end{tabular}

\subsubsection{Media and plates}

In table 16 the instructions for the preparation of media and plates for culturing bacteria, yeast and other cells are given.

Table 16: Media and plates

\begin{tabular}{lll}
\hline Charge /solution & Ingredients & \\
\hline DMEM $(\mathbf{2 0 0} \mathbf{~ m l})$ & $20 \mathrm{ml}$ & FCS \\
(cell culture) & $\underline{2 \mathrm{ml}}$ & $\begin{array}{l}100 \mathrm{x} \\
\text { Penicillin/Streptomycin }\end{array}$ \\
& ad $200 \mathrm{ml} \mathrm{DMEM}$ & \\
\hline $\mathbf{L B}$ medium $(\mathbf{1} \mathbf{~})$ & $10 \mathrm{~g}$ & Peptone \\
(bacteria culture) & $5 \mathrm{~g}$ & Yeast extract \\
& $\underline{10 \mathrm{~g}}$ & $\mathrm{NaCl}$ \\
& ad $1 \mathrm{~L} \mathrm{dd \textrm {H } _ { 2 } \mathrm { O }}$ & \\
\hline & autoclave & \\
\hline
\end{tabular}




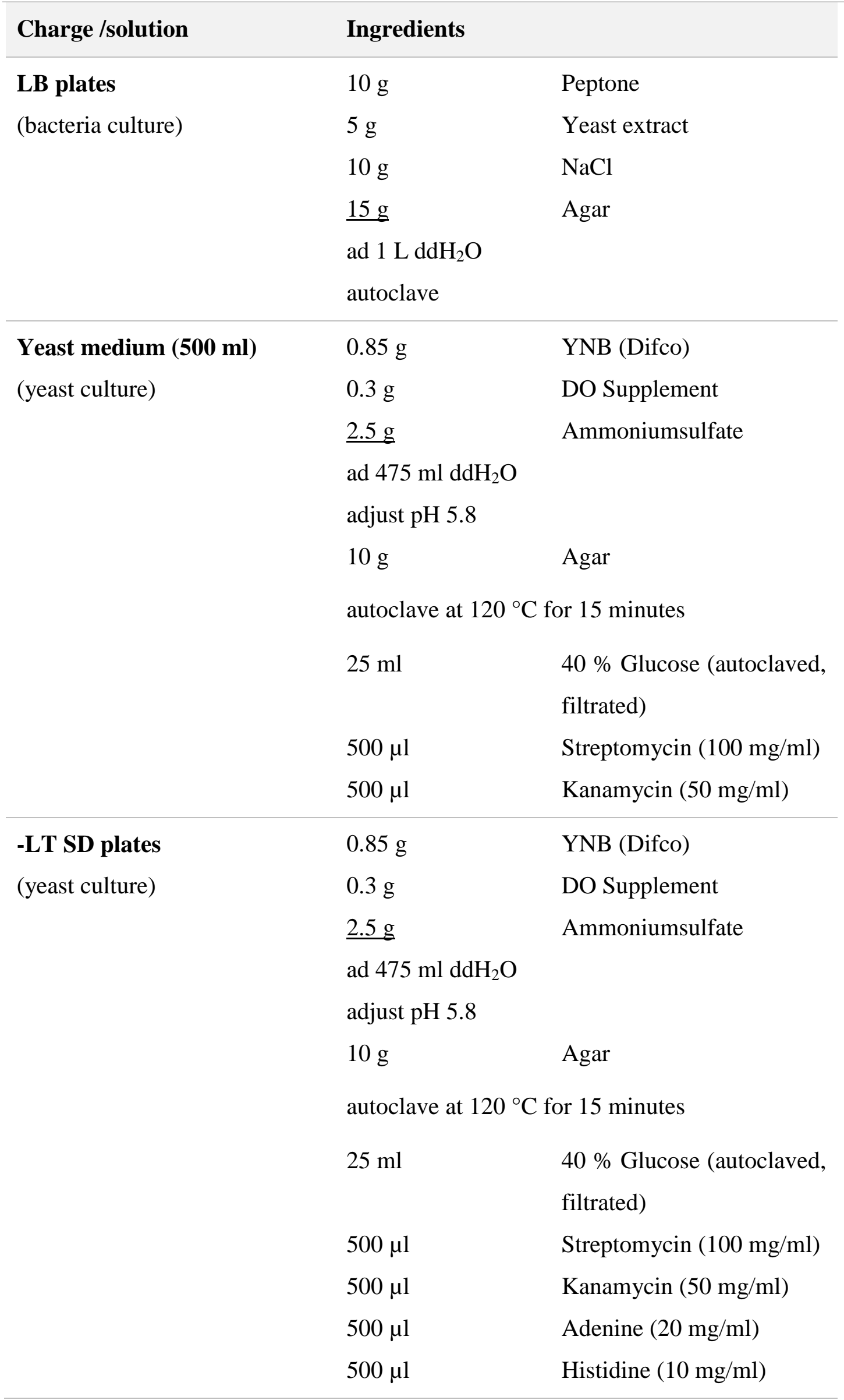


Materials and methods

\begin{tabular}{lll} 
Charge /solution & Ingredients & Charge /solution \\
\hline -LTHA SD plates & $0.85 \mathrm{~g}$ & YNB (Difco) \\
(yeast culture) & $0.3 \mathrm{~g}$ & DO Supplement \\
$2.5 \mathrm{~g}$ & Ammoniumsulfate \\
ad $475 \mathrm{ml} \mathrm{ddH}{ }_{2} \mathrm{O}$ & \\
adjust pH 5.8 & \\
$10 \mathrm{~g}$ & Agar \\
& autoclave at $120^{\circ} \mathrm{C}$ for 15 minutes \\
$25 \mathrm{ml}$ & $40 \%$ Glucose (autoclaved, \\
& filtrated) \\
$500 \mu \mathrm{l}$ & Streptomycin (100 mg/ml) \\
$500 \mu \mathrm{l}$ & Kanamycin $(50 \mathrm{mg} / \mathrm{ml})$ \\
$125 \mu \mathrm{l}$ & Aureobasidin
\end{tabular}

\subsubsection{Sterilisation}

If not indicated differently, sterilisation of solutions and buffers was performed for 20 minutes in the vapour pressure autoclave (Webeco) at $120{ }^{\circ} \mathrm{C}$ at $10^{5} \mathrm{~Pa}$. Heat sensitive solutions were sterile filtrated using filtration units of $0.2-0.45 \mu \mathrm{m}$ pore size. Objects of utility were either autoclaved or heat sterilised for 8-12 hours at $180{ }^{\circ} \mathrm{C}$.

\subsubsection{Antibiotics}

Ampicillin $(50 \mathrm{mg} / \mathrm{ml})$

Aureobasidin A

Kanamycin

Penicillin/Streptomycin $(100 \mathrm{x})$

Streptomycin $(100 \mathrm{mg} / \mathrm{ml})$
AppliChem, Darmstadt

Clontech, Heidelberg

Roth, Karlsruhe

PAN Biotech, Nuremberg

ns

\subsubsection{Antibodies}

All antibodies used in this work are listed in the tables below. Table 17 shows the primary antibodies and table 18 the secondary antibodies. 
Materials and methods

Table 17: Primary antibodies

\begin{tabular}{l|l|l|l}
\hline antibody & origin & dilution & company \\
\hline Anti-HA tag & rat monoclonal & $1: 1,000$ & Roche, Penzberg \\
\hline ASH2L & rabbit polyclonal & $1: 100 / 1: 1,000$ & Abcam, Cambridge (UK) \\
\hline CHD7 & goat polyclonal & $1: 100 / 1: 7,500$ & Abcam, Cambridge (UK) \\
\hline CHD8 & rabbit polyclonal & $1: 100 / 1: 8,500$ & Abcam, Cambridge (UK) \\
\hline HSC-70 & mouse monoclonal & $1: 10,000$ & Santa Cruz, Heidelberg \\
\hline RBBP5 & rabbit polyclonal & $1: 100 / 1: 1,000$ & Abcam, Cambridge (UK) \\
\hline SEMA3D & rabbit polyclonal & $1: 100$ & Santa Cruz, Heidelberg \\
\hline WDR5 & rabbit polyclonal & $1: 100 / 1: 1,000$ & Abcam, Cambridge (UK)
\end{tabular}

Table 18: Secondary antibodies

\begin{tabular}{|c|c|c|c|}
\hline antibody & origin & dilution & company \\
\hline Anti-goat IgG-H\&L (Cy3) & donkey polyclonal & $1: 200$ & $\begin{array}{l}\text { Abcam, } \\
\text { Cambridge (UK) }\end{array}$ \\
\hline $\begin{array}{l}\text { Anti-rabbit IgG (whole } \\
\text { molecule), F }\left(\mathrm{ab}^{\prime}\right) 2 \\
\text { fragment-Cy3 }\end{array}$ & sheep polyclonal & $1: 200$ & $\begin{array}{l}\text { Sigma, } \\
\text { Deisenhofen }\end{array}$ \\
\hline Anti-goat IgG-HRP & donkey polyclonal & $1: 7,500$ & $\begin{array}{l}\text { Santa Cruz, } \\
\text { Heidelberg }\end{array}$ \\
\hline $\begin{array}{l}\text { Anti-mouse IgG }(\mathrm{H}+\mathrm{L})- \\
\text { HRP }\end{array}$ & rabbit polyclonal & $1: 10,0000$ & $\begin{array}{l}\text { Dianova, } \\
\text { Hamburg }\end{array}$ \\
\hline $\begin{array}{l}\text { Anti-rabbit } \operatorname{IgG}(\mathrm{H}+\mathrm{L})- \\
\text { HRP }\end{array}$ & goat polyclonal & $1: 10,0000$ & $\begin{array}{l}\text { Dianova, } \\
\text { Hamburg }\end{array}$ \\
\hline Anti-rat IgG, $(\mathrm{H}+\mathrm{L}) \mathrm{HRP}$ & goat polyclonal & $1: 10,0000$ & $\begin{array}{l}\text { Thermo } \\
\text { Scientific, } \\
\text { Rockford (USA) }\end{array}$ \\
\hline
\end{tabular}

\subsubsection{Morpholinos}

The sequence of the Morpholinos used for injections to knockdown gene expression in Xenopus laevis are listed in table 19.

Table 19: Morpholinos

\begin{tabular}{l|l|l}
\hline name & purpose & sequence \\
\hline Sema3a & blocking of mRNA & ATGCAATCCAGGTCAGAGAGCCCAT \\
\hline Sema3d & blocking of mRNA & GACATTTTTGATGCTTCTCTTTCAT \\
\hline
\end{tabular}




\subsubsection{Oligonucleotides}

The oligonucleotides were purchased from Eurofins Genomics. In table 20 the sequences of the oligonucleotides and their purpose of use are given.

Table 20: Oligonucleotides

\begin{tabular}{|c|c|c|}
\hline name & purpose & sequence \\
\hline ASH2LhY2HF & $\mathrm{Y} 2 \mathrm{H}$ construct & $\begin{array}{l}\text { CCAGATTACGCTCATATGCGCGCGAGAG } \\
\text { AAGAGAGTATT }\end{array}$ \\
\hline ASH2LhY2HR & $\mathrm{Y} 2 \mathrm{H}$ construct & $\begin{array}{l}\text { GGCCTCCATGGCCATATGTTCCCAGAAA } \\
\text { GTCCTTGACAGA }\end{array}$ \\
\hline ASH2L_InFusion_1Rneu & $\mathrm{Y} 2 \mathrm{H}$ construct & $\begin{array}{l}\text { GGCCTCCATGGCCATATGGCATCTTTGG } \\
\text { GAGAACATTTGA }\end{array}$ \\
\hline ASH2L_InFusion_2Rneu & $\mathrm{Y} 2 \mathrm{H}$ construct & $\begin{array}{l}\text { GGCCTCCATGGCCATATGCGGTTGTTGG } \\
\text { CTTATGGTACAC }\end{array}$ \\
\hline DelSema3a1F & patient screen & TGGGCTGGTTAACTAGGATTGTCTGT \\
\hline DelSema3a1R & patient screen & GTTGGGAGGGAGTTCAAGGAATTAAG \\
\hline DelSema3a2F & patient screen & CAATGTTTTGCTTTGTTACCTTGCAG \\
\hline DelSema3a2R & patient screen & ACATACAGCCTACTCCGTTCCTCATC \\
\hline DelSema3a3F & patient screen & СТTTCTTTACTTCAGTTGCCCCAATG \\
\hline DelSema3a3R & patient screen & TCTTCTGGTGTAAGATACTGGCCACA \\
\hline DelSema3a4F & patient screen & CAGTCATGCTGATTGCTGAAACTCTT \\
\hline DelSema3a4R & patient screen & GTTCCACAGGCGTACAAGTGAGTCT \\
\hline DelSema3a5F & patient screen & CCACAGGACAATATTTTTAAGCTGGAG \\
\hline DelSema3a5R & patient screen & GCACCTATTAAAAGGGATGCTGTCA \\
\hline DelSema3a6F & patient screen & GACTTTGCTATCTTCCGAACTCTTGG \\
\hline DelSema3a6R & patient screen & CATCATGAAGTCACCACCATTAGCTT \\
\hline DelSema3a7F & patient screen & AAGTTCATTAGTGCCCACCTCATCTC \\
\hline DelSema3a7R & patient screen & AGCGTGAGTAGCTTTTCCAGAGTGTT \\
\hline DelSema3a8F & patient screen & TCССТTCTTTCAGAATGACTTTGGAG \\
\hline DelSema3a8R & patient screen & ACGCAGTTCATCAAAATGAGTGTCA \\
\hline DelSema3a9F & patient screen & TGTTCAGACTTTGCCATTCCATAAAA \\
\hline DelSema3a9R & patient screen & CCAAGAGTACACAACAGCTCAAAGGTT \\
\hline DelSema3a10F & patient screen & AGGGATCAGCCGTGTGTATGTATAGC \\
\hline DelSema3a10R & patient screen & CTTCCTTGATAAGGCACCCATTGATA \\
\hline DelSema3a11F & patient screen & GAAGTCATCCAGCCATGTACAATCC \\
\hline
\end{tabular}


Materials and methods

\begin{tabular}{|c|c|c|}
\hline name & purpose & sequence \\
\hline DelSema3a11R & patient screen & CATCATACTGTCCATCTTCTGCATCC \\
\hline DelSema3a12F & patient screen & TAACTTGTGGTCTTCTCCCCACTTTC \\
\hline DelSema3a12R & patient screen & AAACTGTCATTTCTTCCAGCAGAACC \\
\hline DelSema3a13F & patient screen & TGTGTCTTATTTCCCAGTCACATTGC \\
\hline DelSema3a13R & patient screen & AAAGCTCCATTGCTGAAATAGCAGTC \\
\hline DelSema3a14F & patient screen & TGTCACAGCAGTAAACTCTTTCCACAG \\
\hline DelSema3a14R & patient screen & TCСТАССТСTTTGCAGTGGGAAAATA \\
\hline DelSema3a15F & patient screen & TTCAGACGCACAAGACGACAAGATA \\
\hline DelSema3a15R & patient screen & TTAGCCTGGTCTTAGCAGGTTGAAAG \\
\hline DelSema3a16F & patient screen & AGCCCTGAAGAGAGAATCATCTATGG \\
\hline DelSema3a16R & patient screen & TCCTGTACCTCTTCTTTTCGCTCTTC \\
\hline DelSema3a17F & patient screen & CTGGAAGTCATTGACACAGAGCATTT \\
\hline DelSema3a17R & patient screen & GAACTCATCCATTGTGTTGAGATTGG \\
\hline Del_SEMA3A_1Fn & patient screen & GACTAAAGCAGCAAAGGGACCTACAG \\
\hline Del_SEMA3A_1Rn & patient screen & GTAGGATAATTTCAGCCTTGGCACAT \\
\hline Del_SEMA3A_9Fn & patient screen & TGTTCAGACTTTGCCATTCCAT \\
\hline Del_SEMA3A_9Rn & patient screen & CAGCTCAAAGGTTAAAGCAACACTT \\
\hline Del_SEMA3A_12Fn & patient screen & TTCAAAGATGTTGGGACCGTTCTTA \\
\hline Del_SEMA3A_12Rn & patient screen & ACTTGTCCATACCAAGTTCAGTGTGC \\
\hline DelSema3d1F & patient screen & CTAAGCATGACCATGTTGTTTCTTCC \\
\hline DelSema3d1R & patient screen & CTGATGCCAAAACATTTCACAGAAG \\
\hline DelSema3d2F & patient screen & GGGTTCATCAGAAGGACTGGATTTT \\
\hline DelSema3d2R & patient screen & ACAAAACGGGAGAAGAAGAGAGATGA \\
\hline DelSema3d3F & patient screen & ATTCACTCTCTGAGCAGAATTGCAGA \\
\hline DelSema3d3R & patient screen & ACTTACATTGGCATCTTTCCCAGCTA \\
\hline DelSema3d4F & patient screen & TGATCTACTTGGAAAACAGGTGGCATA \\
\hline DelSema3d4R & patient screen & CATATTGGATGAAATGCTCCAGTTCC \\
\hline DelSema3d5F & patient screen & AGACTGAAATGTCCTTTCGATCCTCA \\
\hline DelSema3d5R & patient screen & CAATAGGAAAATGCGGTTCAGTCAA \\
\hline DelSema3d6F & patient screen & CATCTTTGGTGTCATGCTGTGAAAT \\
\hline DelSema3d6R & patient screen & TCTGATGTAGTGGTGGTCATGAGTAGG \\
\hline DelSema3d7F & patient screen & TTGGAACTTTCTTCATACCAGACACCT \\
\hline DelSema3d7R & patient screen & CAAACTCTTCCAACTCGAGAAAGGAT \\
\hline
\end{tabular}


Materials and methods

\begin{tabular}{|c|c|c|}
\hline name & purpose & sequence \\
\hline DelSema3d8F & patient screen & TGGAAGTGATGGGGCAGATACTTACT \\
\hline DelSema3d8R & patient screen & CCGTGTACTTTGCGTGTGAATTTTAG \\
\hline DelSema3d9F & patient screen & ACTCCCCACAAGAGATGAAAGAAATC \\
\hline DelSema3d9R & patient screen & ATATGCTACTACAAACGCAGGGACAA \\
\hline DelSema3d10F & patient screen & ATCTTCAAAGGCTCTGCTGTTTGTGT \\
\hline DelSema3d10R & patient screen & CGTGGATAAGGAATTCTCCCATCATA \\
\hline DelSema3d11F & patient screen & ATCAGTTTCATAAAGCGGCACTCTGT \\
\hline DelSema3d11R & patient screen & CATTACATCGTACTGGCCATCTTCTG \\
\hline DelSema3d12F & patient screen & AAGAGAAGTGAGGGGAATGATTTGC \\
\hline DelSema3d12R & patient screen & ACTCCTCCAGCACTACCTCTTCCATA \\
\hline DelSema3d13F & patient screen & CTTGAACATGGAATTGTCTCTGAAGC \\
\hline DelSema3d13R & patient screen & GCAAGGCTGAAACAATGGTCTCTAAT \\
\hline DelSema3d14F & patient screen & ATTGTACATTGGTTCCCGAGATGGAT \\
\hline DelSema3d14R & patient screen & AGTAGGGGTCTCTGGCAAGACAACAG \\
\hline DelSema3d15F & patient screen & GGAGAGCTAGACGCCAAGATGTAAAA \\
\hline DelSema3d15R & patient screen & AATTTCCTGTTGCTCTTCCAGAACAC \\
\hline DelSema3d16F & patient screen & GCTTTCCAGGCATTAGTCATGAAACT \\
\hline DelSema3d16R & patient screen & ATGACAACAGCTTACCTCCTCTCGAT \\
\hline DelSema3d17F & patient screen & CAAAACGGAATATGGGCTACTGATTC \\
\hline DelSema3d17R & patient screen & TATCTCAACCGTGACTCAGCCAATAG \\
\hline Del_SEMA3D_1Fn & patient screen & GACTTAAAGCCAGAAGCCAAGATTTTC \\
\hline Del_SEMA3D_1Rn & patient screen & CCTTTGTAGGTTAGCTTGAGTCTTGGA \\
\hline hCHD7_F1 & $\mathrm{Y} 2 \mathrm{H}$ construct & $\begin{array}{l}\text { GAGGACCTGCATATGAAGATGGCAGATC } \\
\text { CAGGAATGA }\end{array}$ \\
\hline EphA3mF1 & RT-qPCR & AAAGAGGAGGACCCTCCCAGGATGTA \\
\hline EphA3mR1 & RT-qPCR & ATCGGAAGCCTTGTAGAAGCCTGGTC \\
\hline Gfra2mF1 & RT-qPCR & AAACCATCCTGCCCAGCTGTTCCTAT \\
\hline Gfra2mR1 & RT-qPCR & ATTGTCCGGTAGGAGGCTCGACAGTT \\
\hline hCHD7_R1 & $\mathrm{Y} 2 \mathrm{H}$ construct & $\begin{array}{l}\text { CTCCATGGCCATATGCCTTCTGCATCAAC } \\
\text { AGATTCCT }\end{array}$ \\
\hline hCHD7_F2 & $\mathrm{Y} 2 \mathrm{H}$ construct & $\begin{array}{l}\text { AATTCCCGGGGATCCCACCATCTCCTCCT } \\
\text { CCTGAAGAAG }\end{array}$ \\
\hline hCHD7_R2 & $\mathrm{Y} 2 \mathrm{H}$ construct & $\begin{array}{l}\text { CAGGTCGACGGATCCCACTGTAGAGCCT } \\
\text { GGTCTGCTT }\end{array}$ \\
\hline hCHD7_F3 & $\mathrm{Y} 2 \mathrm{H}$ construct & $\begin{array}{l}\text { GAGGACCTGCATATGAAGTGGGCTAAGA } \\
\text { AGGCTGAAT }\end{array}$ \\
\hline hCHD7_R3 & $\mathrm{Y} 2 \mathrm{H}$ construct & $\begin{array}{l}\text { CTCCATGGCCATATGTGTCCTCACTCCCA } \\
\text { CTAATGCT }\end{array}$ \\
\hline
\end{tabular}


Materials and methods

\begin{tabular}{|c|c|c|}
\hline name & purpose & sequence \\
\hline hCHD7_F4 & $\mathrm{Y} 2 \mathrm{H}$ construct & $\begin{array}{l}\text { GAGGACCTGCATATGGAAGCAGTGTTGA } \\
\text { AAGGCAAAT }\end{array}$ \\
\hline hCHD7_R4 & $\mathrm{Y} 2 \mathrm{H}$ construct & $\begin{array}{l}\text { CTCCATGGCCATATGACTTGAACTGGAA } \\
\text { CTGGTACTG }\end{array}$ \\
\hline ifSema3AnewR & cloning & $\begin{array}{l}\text { GGATCCGAGCTCGGTACCACTCAGCTGA } \\
\text { ATTTCCCACCAT }\end{array}$ \\
\hline ifSema3DnewF & cloning & $\begin{array}{l}\text { GCTAGTTAAGCTTGGTACCTAACACCAT } \\
\text { TTGAAAGAGAACATTG }\end{array}$ \\
\hline ifSema3DnewR & cloning & $\begin{array}{l}\text { GGATCCGAGCTCGGTACCCAGATTATTG } \\
\text { TCTTGTGCCTTAGC }\end{array}$ \\
\hline pGADRbBP5F & $\begin{array}{l}\text { cloning in } \\
\text { pGADT7 }\end{array}$ & $\begin{array}{l}\text { CAGATTACGCTCATATGCTCGAGTTGCT } \\
\text { GGAGTCCTT }\end{array}$ \\
\hline pGADRbBP5R & $\begin{array}{l}\text { cloning in } \\
\text { pGADT7 }\end{array}$ & $\begin{array}{l}\text { GCCTCCATGGCCATATGAAAGGGAAGGG } \\
\text { AAGGTCGTA }\end{array}$ \\
\hline pGADWDR51F & $\begin{array}{l}\text { cloning in } \\
\text { pGADT7 }\end{array}$ & $\begin{array}{l}\text { CAGATTACGCTCATATGTCCACCCTTGTC } \\
\text { TCCTGTGC }\end{array}$ \\
\hline pGADWDR51R & $\begin{array}{l}\text { cloning in } \\
\text { pGADT7 }\end{array}$ & $\begin{array}{l}\text { GCCTCCATGGCCATATGGGCAAGAAATG } \\
\text { TCACCAGCA }\end{array}$ \\
\hline pGADWDR52F & $\begin{array}{l}\text { cloning in } \\
\text { pGADT7 }\end{array}$ & $\begin{array}{l}\text { CAGATTACGCTCATATGTTCTGCATCTCG } \\
\text { CTCAACA }\end{array}$ \\
\hline pGADWDR52R & $\begin{array}{l}\text { cloning in } \\
\text { pGADT7 }\end{array}$ & $\begin{array}{l}\text { GCCTCCATGGCCATATGGCAAGAAATGT } \\
\text { CACCAGCAA }\end{array}$ \\
\hline RbBP5pCMV-HA_F & $\begin{array}{l}\text { cloning in } \\
\text { pCMV-HA }\end{array}$ & $\begin{array}{l}\text { CTTATGGCCATGGAGGCCGGGATGAACC } \\
\text { TCGAGTTGCT }\end{array}$ \\
\hline RbBP5pCMV-HA_R & $\begin{array}{l}\text { cloning in } \\
\text { pCMV-HA }\end{array}$ & $\begin{array}{l}\text { ATTCGGGCCTCCATGGCCAAAGGGAAGG } \\
\text { GAAGGTCGTA }\end{array}$ \\
\hline SEMA3A1F & Sequencing & TCACCTGTTACCTCCAGTTTCC \\
\hline SEMA3A1R & Sequencing & GGTTTGATGATTTGGGGTTG \\
\hline SEMA3A2F & Sequencing & TCAGGTTACCATGTACCAACAGTC \\
\hline SEMA3A2R & Sequencing & TGCATCCATTGATTCATTACTGTC \\
\hline SEMA3A3F & Sequencing & ACTTCAGTTGCCCCAATGTC \\
\hline SEMA3A3R & Sequencing & CACATGACCCACAAGTGGAA \\
\hline SEMA3A4F & Sequencing & CAGTCATGCTGATTGCTGAAA \\
\hline SEMA3A4R & Sequencing & TGAACCACAAGCAAAATAAACTG \\
\hline SEMA3A5F & Sequencing & TCTCTGATTAACTGATGTGTTGAAA \\
\hline SEMA3A5R & Sequencing & TTATTTCATAATGGAAAATCTTGGT \\
\hline SEMA3A6F & Sequencing & CATGGTCATAACATGAAACTTGC \\
\hline SEMA3A6R & Sequencing & CCATCATGAAGTCACCACCA \\
\hline SEMA3A7F & Sequencing & TGGACTGTTCAGAATGGTATTATTT \\
\hline SEMA3A7R & Sequencing & CCTGTACCTGTATATTTGACTGCG \\
\hline SEMA3A8/9F & Sequencing & GGAACGATTCGACCACAAAT \\
\hline SEMA3A8/9R & Sequencing & TGCAAATTATGAGTACTTGGATAGC \\
\hline SEMA3A10F & Sequencing & GCCATTTTCACCTATGCCTT \\
\hline SEMA3A10R & Sequencing & TCTGTCTGTAGCTGCATTGTTTT \\
\hline
\end{tabular}


Materials and methods

\begin{tabular}{|c|c|c|}
\hline name & purpose & sequence \\
\hline SEMA3A11F & Sequencing & GAACCATTGAGGCCATGTGT \\
\hline SEMA3A11R & Sequencing & CCAACCCCTGAGATGTTCAA \\
\hline SEMA3A12F & Sequencing & AAAAGGAAGACCGATATCAAAGG \\
\hline SEMA3A12R & Sequencing & TGAGAAAACAAAATATGAGCCAAA \\
\hline SEMA3A13F & Sequencing & AGCAATAACCCCAACTTGGTC \\
\hline SEMA3A13R & Sequencing & ATCAAAAACATGAGGGCAATG \\
\hline SEMA3A14F & Sequencing & AGAAGGCCTTTAAAGAAATTAGCA \\
\hline SEMA3A14R & Sequencing & TTGATGCACTTATTTGAAGAAAGC \\
\hline SEMA3A15F & Sequencing & TCTGGTAGTGAAAAAGCCATGA \\
\hline SEMA3A15R & Sequencing & TCTCTTCGGCTGCATTTCTT \\
\hline SEMA3A16F & Sequencing & TGGCAATAACTTGTCTCCTGAA \\
\hline SEMA3A16R & Sequencing & TGAATGAGCGATTGATTGGT \\
\hline SEMA3A17F & Sequencing & ACAGACACGGAGTTTCAGAGC \\
\hline SEMA3A17R1 & Sequencing & AGATTGGGGTGGTTGATGAG \\
\hline SEMA3A17R2 & Sequencing & CCCACCATTGTAAACATCCA \\
\hline SEMA3D1F & Sequencing & AACACCATTTGAAAGAGAACATTG \\
\hline SEMA3D1R & Sequencing & AATGAGTAATTGGGCTTCGTGT \\
\hline SEMA3D2F & Sequencing & TTGGCCCTTTTATGCTGTATG \\
\hline SEMA3D2R & Sequencing & CCAGAATGTGTATCAGAGGCAAT \\
\hline SEMA3D3F & Sequencing & TCTGAGCAGAATTGCAGAGTG \\
\hline SEMA3D3R & Sequencing & CCATTAATTCAGTAAAGCCCAAT \\
\hline SEMA3D4F & Sequencing & АTTTGTCTTGCCTCGTTTGC \\
\hline SEMA3D4R & Sequencing & TCAAATCTGCCTGTTTTATGTCA \\
\hline SEMA3D5F & Sequencing & GGAATTTAGTTTGTATCTCCCACA \\
\hline SEMA3D5R & Sequencing & AATAATGGAAACAAATCGCTTG \\
\hline SEMA3D6F & Sequencing & GGTGTCATGCTGTGAAATTTGT \\
\hline SEMA3D6R & Sequencing & TTAAACCAAAGCAAGACAATCAAA \\
\hline SEMA3D7F & Sequencing & CCCACATCTGGCTTATAGTTCA \\
\hline SEMA3D7R & Sequencing & AGCATTAACTTTGGCTTAAACCTT \\
\hline SEMA3D8Fa & Sequencing & TGATGGTAGTGGTGCGTGTATT \\
\hline SEMA3D8Ra & Sequencing & TGCATTGTTCAAATCATTTATGC \\
\hline SEMA3D8Fb & Sequencing & AGACACTACCTGTAATAGCCACCA \\
\hline SEMA3D8Rb & Sequencing & TGGAGCCAAAGGGAAATAAA \\
\hline
\end{tabular}


Materials and methods

\begin{tabular}{|c|c|c|}
\hline name & purpose & sequence \\
\hline SEMA3D9F & Sequencing & GCATAATATAGTGCCTCTGTTAATCG \\
\hline SEMA3D9R & Sequencing & CTACTACAAACGCAGGGACAAA \\
\hline SEMA3D10F & Sequencing & TGAATGATTGAATGATGATGCT \\
\hline SEMA3D10R & Sequencing & GTGGCTCTGGTGAATAGCAG \\
\hline SEMA3D11F & Sequencing & TTCCATATTGACTCTGTTGTCCA \\
\hline SEMA3D11R & Sequencing & TTTTAAGAAATGCTGACAAGGTTTT \\
\hline SEMA3D12F & Sequencing & GAGAAGTGAGGGGAATGATTTG \\
\hline SEMA3D12R & Sequencing & AAACAAGGGTGCCCTATAACAA \\
\hline SEMA3D13F & Sequencing & TTTACTTCAGTGATATATGGCATCAG \\
\hline SEMA3D13R & Sequencing & TTTAACTCCCAATTTCAATGGAT \\
\hline SEMA3D14F & Sequencing & GTCTGAAAGATGCTTCTAATTCATTT \\
\hline SEMA3D14R & Sequencing & TTCCTTCCATAGGACATGTTAGTAAT \\
\hline SEMA3D15F & Sequencing & CATAGTACTGCATCTGCCACTGT \\
\hline SEMA3D15R & Sequencing & CCAGAACACATGCATTACACAA \\
\hline SEMA3D16F & Sequencing & TGGCATCTGTTCTGAATCAGTC \\
\hline SEMA3D16R & Sequencing & TCAATCGTACACTATTTCCCTCAA \\
\hline SEMA3D17F1 & Sequencing & CATTTGCTCTTAGCTCTCTCTGTG \\
\hline SEMA3D17R1 & Sequencing & TCATCCAGGTCTCTGTGATGTC \\
\hline SEMA3D17F2 & Sequencing & AAATCCTTAGCAGCCCAAACTT \\
\hline SEMA3D17R2 & Sequencing & ACTCCATGGGAAGCATTTATGA \\
\hline SeqhASH2L1F & Sequencing & GGTCGCAAATGCAACAGG \\
\hline SeqhASH2L1R & Sequencing & GCTTACATCGACCAAGTTTGC \\
\hline SeqhASH2L2F & Sequencing & ACAGGGACCACCAAGAAGG \\
\hline SeqhASH2L2R & Sequencing & CCATCTTTGTTAAACGGGTGTT \\
\hline SeqhASH2L3F & Sequencing & CCATTAACTTTGGACCATGCTT \\
\hline SeqhASH2L3R & Sequencing & ATGTCACTCATAGGGCGGTAAG \\
\hline SeqCHD71.1R & Sequencing & ACTGTCTGGCTCCGAGAACTAA \\
\hline SeqCHD71.2F & Sequencing & GCCTCAATCAGGGAAATCCT \\
\hline SeqCHD71.2R & Sequencing & CAGGTATCAGTCGTTCCTGGAT \\
\hline SeqCHD71.3F & Sequencing & ATCCTCAGCCATCTCACCAG \\
\hline SeqCHD71.4F & Sequencing & TAGCAGAGGATCCCAGTAAAGG \\
\hline SeqCHD72.1R & Sequencing & CTGGAGACTCTGCATTGTGTGT \\
\hline SeqCHD72.2F & Sequencing & GGTGGTCAAGCTAACGTACCTAAC \\
\hline
\end{tabular}


Materials and methods

\begin{tabular}{|c|c|c|}
\hline name & purpose & sequence \\
\hline SeqCHD73.1R & Sequencing & СTCTCACTGTGCTTACCTGTGG \\
\hline SeqCHD73.2F & Sequencing & GAACAGACATGCTAGCAGATGG \\
\hline SeqCHD73.2R & Sequencing & GGATAACTCAGGGTCATTGAGG \\
\hline SeqCHD74.1R & Sequencing & GGCCTTCTTCCTCTTCTGCT \\
\hline SeqCHD74.2F & Sequencing & CAATGAAGGATCTACCCAGGTG \\
\hline seqCHD7_1vectR & Sequencing & GCTGACCCATAGGATTTACTGG \\
\hline seqCHD7_2vectR & Sequencing & TCCAACTGGTATTCCCTGAGTT \\
\hline seqCHD7_3vectR & Sequencing & GATGGTTCTGCAGATGGTTTCT \\
\hline seqCHD7_4vectR & Sequencing & ССТСТСТTGAGGCCTTTGATAA \\
\hline SeqCHD7F1new & Sequencing & ATGGCAGATCCAGGAATGAT \\
\hline SeqCHD7F2new & Sequencing & AGATCAAGCAAAGATCGAGGAG \\
\hline SeqCHD7F3new & Sequencing & AАCCTCACTGCTGCTGATACCT \\
\hline SeqCHD7F4new & Sequencing & AATGTTTCAGGGTGGAGAAGAA \\
\hline SeqCHD7F5new & Sequencing & CTTGGATCTGCCAGAGTGGT \\
\hline SeqCHD7F6new & Sequencing & CAAGAGGCGAAATCTCATGG \\
\hline SeqCHD7F7new & Sequencing & CTAGTCAAGGAGAACCGGAAGA \\
\hline seqRbBP5h.F4 & Sequencing & GACCCTATTGCTGCCTTCTGTA \\
\hline SeqxSema3a1F & Sequencing & CTGACCTGGATTGCATTTCTTT \\
\hline SeqxSema3a1R & Sequencing & TTGTAGGACAATCTGAGCCTTG \\
\hline SeqxSema3a2F & Sequencing & GGTGGCCACAGAAGCTTAGTTA \\
\hline SeqxSema3a2R & Sequencing & ACATTCTGACAGTTGAGCCTTG \\
\hline SeqxSema3a3F & Sequencing & AGCTGAGGATGGTCAATACGAT \\
\hline SeqxSema3a3R & Sequencing & TGTATCAATGCCATTAGGACCA \\
\hline SeqxSema3a4F & Sequencing & AATGTCCAGGTTAGCAACACAA \\
\hline SeqxSema3a4R & Sequencing & СТCAAATTCATGGGTCCTCCTA \\
\hline seqSEMA3A_F3new & Sequencing & AGACGCACAAGACGACAAGATA \\
\hline seqSEMA3A_R3new & Sequencing & АТTCTCTCTTCAGGGCTGTGG \\
\hline SeqSema3d_1F & Sequencing & TTAAACCGCAGCATTCCTCTAC \\
\hline SeqSema3d_1R & Sequencing & CCGAGGAATATTCTGTTTCAGG \\
\hline SeqSema3d_2F & Sequencing & TGTTCAATTCCTGGACAAGATG \\
\hline SeqSema3d_2R & Sequencing & GGATTTCTTTCGTCCTTTGTTG \\
\hline SeqSema3d_3F & Sequencing & CAATCAGGTGGTTTATTCAGCA \\
\hline SeqSema3d_3R & Sequencing & GAATCAGAAGCCCGTAGTCTGT \\
\hline
\end{tabular}


Materials and methods

\begin{tabular}{l|l|l}
\hline name & purpose & sequence \\
\hline Sema3AmF1 & RT-qPCR & CGGGACTTCGCTATCTTCAGAACACT \\
\hline Sema3AmR1 & RT-qPCR & TCATCTTCAGGGTTGTCACTCTCTGG \\
\hline Sema3DmF1 & RT-qPCR & TTACGTGTGTGGAACTGGAGCGTTTC \\
\hline Sema3DmR1 & RT-qPCR & AGGCTGCTGAGGATCAAAGGGACAT \\
\hline SOX10mF1 & RT-qPCR & GCTCAGCAAGACACTAGGCAAGCTCT \\
\hline SOX10mR1 & RT-qPCR & CGAGGTTGGTACTTGTAGTCCGGATG \\
\hline SRY_F & gender PCR & AGCCTGTTGATATCCCCACTG \\
\hline SRY_R & gender PCR & ATGCTCACCAGTGTGTCAGC \\
\hline Trp53bp2mF1 & RT-qPCR & CCCAGTTGCTGATAACGAACGGATGT \\
\hline Trp53bp2mR1 & RT-qPCR & TCGGTCCACTCACAATGTCCCTGTTA \\
\hline WDR5hY2HF & Y2H construct & $\begin{array}{l}\text { CCAGATTACGCTCATATGTCCACCCTTGT } \\
\text { CTCCTGTGC }\end{array}$ \\
\hline WDR5hY2HR & Y2H construct & GCGCTCCATGGCCATATGGGTCAACTTC \\
\hline
\end{tabular}

\subsubsection{DNA marker}

100 bp DNA ladder

Invitrogen, Karlsruhe

$1 \mathrm{~kb}$ DNA ladder

Invitrogen, Karlsruhe

$1 \mathrm{~kb}$ DNA ladder

BioLabs, Frankfurt am Main

\subsubsection{Protein marker}

See Blue Plus 2 Pre-stained Standard

Invitrogen, Karlsruhe

Hi Mark Pre-Stained

Invitrogen, Karlsruhe

\subsubsection{Vectors}

pBluescript SK (-)

Agilent Technologies,

Boeblingen

pcDNA3.1 (+) Flag-6xHis

Invitrogen, Karlsruhe

pCMV-HA

Clontech, Heidelberg

pCMV-Myc

Clontech, Heidelberg

pCS2+MT

ns

pGADT7

Clontech, Heidelberg

pGBKT7

Clontech, Heidelberg

pGEM-T Easy

Promega, Mannheim 


\subsubsection{Enzymes}

Antarctisc Phosphortase

DNase I (1 U/ $\mu \mathrm{l})$

Proteinase $\mathrm{K}$

SuperScript ${ }^{\circledR}$ II

T4 Ligase

\subsubsection{Polymerases (Kits)}

IMMOLASE $^{\mathrm{TM}}$ DNA Polymerase

In-Fusion ${ }^{\mathrm{TM}}$ Advantage PCR Cloning Kit

peqGOLD $P w o$-DNA-Polymerase

PfuUltra ${ }^{\mathrm{TM}}$ Hi-Fidelity DNA polymerase

Platinum ${ }^{\circledR}$ Taq DNA Polymerase

\subsubsection{Restriction enzymes and Buffers}

BamHI

EcoRI

$M s c \mathrm{I}$

NdeI

NruI

SfiI

XhoI

H Buffer

NEBuffer 2

NEBuffer 3

NEBuffer 4

React3

\subsubsection{Bacterial strains}

E. coli $\mathrm{DH} 5 \alpha$ competent cells

Stellar competent cells
BioLabs, Frankfurt am Main

Thermo Scientific, Schwerte

Roth, Karlsruhe

Invitrogen, Karlsruhe

BioLabs, Frankfurt am Main

Bioline, Luckenwalde

Clontech, Heidelberg

Peqlab, Erlangen

Stratagene, La Jolla (USA)

Invitrogen, Karlsruhe

Invitrogen, Karlsruhe

Invitrogen, Karlsruhe

BioLabs, Frankfurt am Main

BioLabs, Frankfurt am Main

BioLabs, Frankfurt am Main

BioLabs, Frankfurt am Main

Invitrogen, Karlsruhe

Invitrogen, Karlsruhe

Invitrogen, Karlsruhe

BioLabs, Frankfurt am Main

BioLabs, Frankfurt am Main

Invitrogen, Karlsruhe

Invitrogen, Karlsruhe

Clontech, Heidelberg 


\subsubsection{Cell line}

HeLa cells (human)

\subsubsection{Yeast strain}

Saccharomyces cerevisiae AH109

Clontech, Heidelberg

Y2HGold Yeast Strain

Clontech, Heidelberg

\subsubsection{Model organisms}

The Whirligig mouse line was generated by ENU (N-ethyl-N-nitrosourea) mutagenesis (Bosman et al., 2005). It was kindly provided from K. P. Steel (Sanger Centre, Cambridge, United Kingdom) and the Helmholtz Zentrum Munich, Germany.

African clawed frog Xenopus laevis. Adult frogs were purchased from Nasco (Ft. Atkinson, WI, USA).

\subsubsection{Software used}

Adobe Photoshop SC4 (compile figures)

AlphaView (capturing and analysing images)

Gen5 ${ }^{\mathrm{TM}}$ (analysing data, determining protein concentration)

Microsoft Office Excel 2007 (analysing data)

Microsoft Office PowerPoint 2007 (compile figures/tables)

Microsoft Office Word 2007 (compile documents)

Paint (Erstellen und Bearbeiten von Zeichnungen)

SDS Version 2.1, PE Applied Biosystems (analysing RT-qPCR)

4D Tierbase (administration of mice)

Cellsens Dimension (microscopy imaging applications)

\subsubsection{Internet platforms used}

BLAST (Analysis of DNA sequences)

http://blast.ncbi.nlm.nih.gov/Blast.cgi

dbSNP (information about mutations)

http://www.ncbi.nlm.nih.gov/projects/SNP/ 
Ensembl (genetic information)

http://www.ensembl.org/index.html

Mutation taster (prediction program)

http://www.mutationtaster.org/

NCBI, National Center for Biotechnology Information (biomedical and genetic information)

http://www.ncbi.nlm.nih.gov/

NEBcutter V2.0 (recognition sites for restriction enzymes)

http://tools.neb.com/NEBcutter2/index.php

OMIM (information about genes and diseases)

http://www.ncbi.nlm.nih.gov/omim

PolyPhen2 (prediction program)

http://genetics.bwh.harvard.edu/pph2/

Primer3 Input (version 0.4.0) (searching for oligonucleotides)

http://frodo.wi.mit.edu/primer3/

Pubmed (searching for publications)

http://www.ncbi.nlm.nih.gov/pubmed/

RepeatMasker Web Server (checking for repetitive sequences within genes) http://www.repeatmasker.org/cgi-bin/WEBRepeatMasker

SIFT (prediction program)

http://sift.jcvi.org/ 


\subsection{Methods}

\subsubsection{Isolation of nucleic acids}

\subsubsection{Isolation of genomic DNA for genotyping of mice}

To determine the genotype of mouse litters, a tail biopsy was taken at the age of three weeks. To isolate genomic DNA from mouse tail, it was proceded according to Wieczerzak (2012) with a modified centrifugation step. Therefore, $150 \mu 1$ of direct lysis buffer (Peqlab) and $5 \mu \mathrm{l}$ Proteinase $\mathrm{K}(10 \mu \mathrm{g} / \mathrm{ml})$ were added and incubated overnight at $55{ }^{\circ} \mathrm{C}$ under shaking. Inactivation of proteinase $\mathrm{K}$ took place at $85{ }^{\circ} \mathrm{C}$ for 50 minutes and the samples were centrifuged at 13,000 rpm for 1 minute. Probes were kept at $8{ }^{\circ} \mathrm{C}$ until proceeding with the genotyping PCR using Immolase ${ }^{\mathrm{TM}}$ DNA polymerase (compare 2.2.7).

\subsubsection{Isolation of plasmid DNA from bacteria}

The plasmid preparation is a procedure to isolate and purify plasmid DNA from bacteria. Three basic steps can be mentioned:

$>$ preparation of a bacterial culture

$>$ lyse the bacteria to extract plasmid DNA

$>$ purification of plasmid DNA

\section{a) Mini-preparation}

To extract and purify moderate yields of plasmid DNA from bacteria, the QIAprep ${ }^{\circledR}$ Miniprep from Qiagen was used following the company's instructions with some modifications regarding the amount of the buffers and incubation times. The principle of this procedure is based on a modified alkaline lysis method of Birnboim and Doly (1979). Starting material is a $5 \mathrm{ml}$ overnight culture of E. coli in LB (Lysogeny Broth) medium with $5 \mu \mathrm{l}$ of an appropriate antibiotic $(50 \mathrm{mg} / \mathrm{ml})$. First, the bacterial cells were pelleted by centrifugation at 4,000 rpm for 10 minutes at $8{ }^{\circ} \mathrm{C}$. The supernatant was discarded and the pellet resuspended in $200 \mu 1$ Buffer P1 (Resuspension Buffer) with RNase A (Qiagen protocol: $250 \mu$ l). Next, $200 \mu$ l Buffer P2 (Lysis Buffer) was added and mixed thoroughly by inverting the tube 4-6 times (Qiagen protocol: $250 \mu \mathrm{l}$ ). After incubation for 5 minutes at room temperature, $200 \mu 1$ Buffer N3 (Neutralisation Buffer) was added (Qiagen protocol: $350 \mu 1$ ). The 
solution was immediately mixed by inverting the tubes 4-6 times. Samples were incubated for 5 minutes on ice then centrifuged at $13,000 \mathrm{rpm}$ for 20 minutes at $4{ }^{\circ} \mathrm{C}$ (Qiagen protocol: no incubation before the 10 minutes centrifugation step at room temperature). In this protocol no column is utilised; instead, the plasmid DNA is precipitated with isopropanol. The supernatant was carefully transferred to a new tube without taking debris from the pellet. To precipitate the DNA, $420 \mu 1$ isopropanol was added, mixed and samples incubated for 15 minutes at room temperature before centrifuging at $13,000 \mathrm{rpm}$ for 30 minutes at $4{ }^{\circ} \mathrm{C}$. The supernatant was discarded and the DNA pellet washed with $500 \mu 170 \%$ ethanol. After a final centrifugation step at $13,000 \mathrm{rpm}$ for 5 minutes at $4{ }^{\circ} \mathrm{C}$, the supernatant was discarded and the pellet air-dried. Depending on its size the DNA pellet was dissolved in 30-50 $\mu \mathrm{l}$ TE (Tris-EDTA) Buffer or $\mathrm{ddH}_{2} \mathrm{O}$. The DNA concentration was measured with the photometer (Eppendorf) (compare 2.2.2) and stored at $-20{ }^{\circ} \mathrm{C}$.

\section{b) Midi-preparation}

This method is used to isolate and purify plasmid DNA from bacteria. Here, the PureLink $^{\circledR}$ HiPure Plasmid Filter Purification Kit (for midi and maxi preparation of plasmid DNA) from Invitrogen was used which employs a patented anion-exchange resin that ensures high yields of highly pure plasmid DNA. The midiprep was performed according to the manufacturer's instructions with slightly modifications. First, the cell lysate was prepared. Departing from the manufacturer's advice $30 \mathrm{ml}$ instead of 15-25 $\mathrm{ml}$ of an overnight LB culture per sample was used for high copy number plasmids containing $30 \mu \mathrm{l}$ of an appropriate antibiotic $(50 \mathrm{mg} / \mathrm{ml})$. The cells were harvested by centrifuging at 4,000 $\mathrm{x} g$ for 10 minutes at room temperature. Next, $4 \mathrm{ml}$ Resuspension Buffer (R3) with RNase A was added to the cell pellet which was vortexed until cells were homogenously resuspended (Invitrogen protocol: $10 \mathrm{ml} \mathrm{R} 3$ buffer). After that $4 \mathrm{ml}$ Lysis Buffer (L7) was added and tubes were inverted until the lysate composite was homogenously mixed (Invitrogen protocol: $10 \mathrm{ml} \mathrm{L7} \mathrm{buffer).} \mathrm{Next,} \mathrm{the} \mathrm{lysate} \mathrm{was} \mathrm{incubated} \mathrm{at} \mathrm{room} \mathrm{temperature} \mathrm{for}$ exactly 5 minutes. Then, $4 \mathrm{ml}$ Precipitation Buffer (N3) was added and tubes were homogeneously mixed by inverting (Invitrogen protocol: $10 \mathrm{ml} \mathrm{N} 3$ buffer). While probes were centrifuging at 12,000 x $g$ for 10 minutes at room temperature (not listed in the manufacturer's protocol), the columns were equilibrated by applying $10 \mathrm{ml}$ Equilibration Buffer (EQ1) directly to the filtration cartridge of the column 
(Invitrogen protocol: $15 \mathrm{ml}$ EQ1 buffer). After the solution drained by gravity flow, the precipitated lysate was transferred onto the column through a filter to prevent loading any protein remains. The column was washed twice with $10 \mathrm{ml}$ Washing Buffer (W8). The buffer flew through the column by gravity flow until the flow stopped. The flow through was discarded and the DNA was eluted. Therefore, a sterile tube was placed under the column and $5 \mathrm{ml}$ Elution Buffer (E4) was applied to the column. The solution was drained by gravity flow. Next, the DNA was precipitated by adding $3.5 \mathrm{ml}$ isopropanol to the elution, then mixed well and incubated for 2 minutes at room temperature. A centrifugation step was performed at $>12,000 \times \mathrm{g}$ for 30 minutes at $4{ }^{\circ} \mathrm{C}$. The supernatant was removed and the pellet washed with $3 \mathrm{ml} 70 \%$ ethanol. After centrifuging at $>12,000 \times g$ for 5 minutes at $4{ }^{\circ} \mathrm{C}$, the supernatant was removed and the pellet was air-dried for about 10 minutes. Depending on the pellet size the DNA was resuspended in 100-200 $\mu 1$ TE Buffer or $\mathrm{ddH}_{2} \mathrm{O}$. The concentration of the precipitated DNA was measured with a spectral photometer (Eppendorf) (compare 2.2.2) and stored at $-20^{\circ} \mathrm{C}$.

\subsubsection{RNA isolation from mouse embryos}

For the RNA isolation E9.5 old Whirligig mouse embryos (wild-type $\left(C h d 7^{+/+}\right)$, heterozygous $\left(C h d 7^{W h i /+}\right)$ and homozygous $\left(C h d 7^{W h i / W h i}\right)$ ) were used according to the standard protocol of the Transcriptome Analysis Laboratory (TAL), Goettingen. Adding $1 \mathrm{ml}$ TRizol Reagent (Invitrogen) the entire embryo was lysed in a rotor homogenisator for 5 minutes at 50 oscillations. Next, the homogenate was incubated for 5 minutes at room temperature. To remove insoluble material from the homogenate, samples were centrifuged at $12,000 \times g$ for 10 minutes at $4{ }^{\circ} \mathrm{C}$. The supernatant was transferred to a fresh tube and the sample incubated again for 5 minutes at room temperature. $200 \mu$ l chloroform was added, tubes were vigorously shaken by hand for 15 seconds, then incubated 5 minutes at room temperature. After a 15 minute centrifugation step at $12,000 \times g$ at $4{ }^{\circ} \mathrm{C}$, the upper aqueous phase containing the RNA was transferred to a fresh $2 \mathrm{ml}$ tube. Next, the RNA was precipitated by adding $500 \mu \mathrm{l}$ isopropanol and $1 \mu$ l GlycoBlue. Samples were vortexed and incubated at $-20{ }^{\circ} \mathrm{C}$ for 2 hours or overnight. The samples were centrifuged at $12,000 \times \mathrm{g}$ for 30 minutes at $4{ }^{\circ} \mathrm{C}$. The supernatant was removed and the RNA pellet was washed with $1 \mathrm{ml} 75 \%$ ethanol. Samples were centrifuged at $12,000 \times \mathrm{g}$ for 5 minutes at $4{ }^{\circ} \mathrm{C}$ and the supernatant discarded. In case of a DNase 
treatment a repetition of the wash step is not necessary. The RNA pellet was air-dried and dissolved in $25 \mu \mathrm{l}$ DEPC. The concentration of the RNA was determined using a photo spectrometer (Eppendorf) (compare 2.2.2) and stored at $-80^{\circ} \mathrm{C}$.

\subsubsection{Determination of nucleic acid concentration}

To measure the concentration of DNA or RNA, a spectral photometer (Eppendorf) was used as described by Nolte (2008). After determining the blank value (solvent, e.g., TE Buffer, DEPC or $\mathrm{ddH}_{2} \mathrm{O}$ ) the concentration of the nucleic acid could be calculated by measuring the absorbance at $260 \mathrm{~nm}$ on the supposition that $\mathrm{E}_{260}$ of a $50 \mathrm{ng} / \mu \mathrm{l}$ DNA solution (and $40 \mathrm{ng} / \mu \mathrm{l}$ of a RNA solution) equals 1 . The additional quotients $\mathrm{E}_{260} / \mathrm{E}_{280}$ and $\mathrm{E}_{260} / \mathrm{E}_{230}$ reflect contaminations with proteins and salt, respectively. The values should be $>1.8$ and $>2.0$, respectively. Before the measurement the type of nucleic acid and the dilution factor of the sample were quoted and the concentration in $\mu \mathrm{g} / \mu \mathrm{l}$ was determined.

\subsubsection{Reverse transcription}

This method was applied to transcribe RNA into complementary DNA (cDNA) using SuperScript II reverse transcriptase (Invitrogen) according to the manufacturer's instructions. In total, $5 \mu \mathrm{g}$ RNA was used for a total reaction volume of $20 \mu \mathrm{l}$. Next, $1 \mu \mathrm{l}$ Oligo dT $(500 \mu \mathrm{g} / \mathrm{ml})$ and $1 \mu \mathrm{dNTP}$ mix (10 mM each) were added to the RNA and $\mathrm{ddH}_{2} \mathrm{O}$ was admitted up to $12 \mu \mathrm{l}$ total. The mixture was incubated at $65{ }^{\circ} \mathrm{C}$ for 5 minutes then $2 \mu 1$ of $0.1 \mathrm{M}$ DTT and $4 \mu \mathrm{l} 5 \mathrm{x}$ First-Strand Buffer were added and mixed gently. Next, samples were incubated at $42{ }^{\circ} \mathrm{C}$ for 2 minutes before adding $1 \mu 1$ of SuperScript II reverse transcriptase. The reaction sample was gently mixed and incubated at $42{ }^{\circ} \mathrm{C}$ for 50 minutes. By heating up to $70{ }^{\circ} \mathrm{C}$ for 15 minutes the reaction was inactivated. The cDNA was kept on ice for 5 minutes and stored at $-20^{\circ} \mathrm{C}$. To verify whether the transcription was successful, a PCR was performed (compare 2.2.7).

\subsubsection{Cloning}

Cloning is a method used to create copies of specific DNA fragments. The target DNA is brought into bacterial cells via plasmids. Through cell division (formation of bacterial colonies) the DNA is multiplied. 


\section{Materials and methods}

\subsubsection{Restriction digestion of plasmid DNA}

Restriction endonucleases are enzymes which naturally occur in bacteria and archaea where they have a protecting function against virus invasion (Arber and Linn, 1969; Pingoud et al., 1993). The restriction endonucleases used in this work belong to the type II restriction enzymes. They recognise a specific palindromic sequence of nucleotides and cleave the DNA at the same site (Pingoud and Jeltsch, 2001).

Plasmid DNA was cleaved using restriction enzymes from different companies. In general 10 units (U) enzyme were used to cleave $1 \mu \mathrm{g}$ DNA in 1-2 hours. The incubation temperature as well as the reaction Buffer depends on the enzyme. To some restriction enzymes 1 x Bovine Serum Albumin (BSA) was added to prevent unspecific bindings. To check whether the cleavage was complete, an aliquote of the reaction was put on an agarose gel (compare 2.2.10).

\subsubsection{Ligation of DNA fragments into plasmids}

For the ligation target DNA fragments (inserts) were amplified by PCR. Inserts and the desired plasmid were digested with appropriate restriction enzymes. For the ligation the T4 Ligase (BioLabs) was used according to the manufacturer's instructions. A reaction volume of $10 \mu \mathrm{l}$ contained a molar ratio of 1:3 vector to insert, $1 \mu 1 \mathrm{~T} 4$ ligase and $2 \mu \mathrm{l} 5 \mathrm{x}$ Ligation Buffer. If needed, $\mathrm{ddH}_{2} \mathrm{O}$ was supplied to a final volume of $10 \mu 1$. The ligation was incubated overnight at $4{ }^{\circ} \mathrm{C}$.

\subsubsection{Transformation}

For the transformation of a ligation reaction, $50 \mu$ l competent E.coli DH5 $\alpha$-cells (Invitrogen) were defrozen on ice and proceeded according to Hanahan (1983) with some modifications. $10 \mu \mathrm{l}$ of the ligation mix was carefully added to the DH $5 \alpha$-cells and incubated for 30 minutes on ice. To increase the rate of plasmids adsorbed by the cells, a heat shock was performed at $42{ }^{\circ} \mathrm{C}$ for 45 seconds instead of 90 seconds. After that, the samples were incubated on ice for 2 minutes. For proliferation of bacterial cells, $450 \mu \mathrm{l}$ prewarmed S.O.C. (Super Optimal Broth) medium (Invitrogen) instead of $800 \mu \mathrm{l}$ was supplied and the samples incubated under continuously shaking at $37{ }^{\circ} \mathrm{C}$ for 1 hour. $100 \mu \mathrm{l}$ of the bacterial mixture was plated on LB plates with an appropriate antibiotic. The plates were incubated overnight at $37^{\circ} \mathrm{C}$. 
For a transformation of an In-Fusion ${ }^{\mathrm{TM}}$ reaction in DH5 $\alpha$-cells, 2.5-3 $\mu$ l of the reaction was used and proceeded as described previously.

If an In-Fusion ${ }^{\mathrm{TM}}$ reaction was transformed into Stella cells, $2.5-3 \mu 1$ of the mixture was used, respectively and proceeded as described previously. After 1 hour incubation at $37{ }^{\circ} \mathrm{C}, 50 \mu \mathrm{l}$ of the cell culture was mixed with $50 \mu \mathrm{l}$ fresh S.O.C. medium and plated on an LB plate with an appropriate antibiotic. The other $400 \mu 1$ S.O.C. medium was centrifuged at $6,000 \mathrm{rpm}$ for 5 minutes at room temperature. The supernatant was discarded and the cell pellet resuspended in $100 \mu \mathrm{l}$ fresh S.O.C. medium, then plated on an LB plate with an appropriate antibiotic and incubated overnight at $37^{\circ} \mathrm{C}$.

\subsubsection{Cloning by In-Fusion ${ }^{\mathrm{TM}}$}

In-Fusion $^{\mathrm{TM}}$ is a PCR cloning method typically used to clone PCR products into vectors. In this work the In-Fusion ${ }^{\mathrm{TM}}$ Advantage PCR Cloning Kit (Clontech) was used according to the company's manual. First, gene-specific In-Fusion primers were designed with 15 bases extensions homologous to the ends of the linearised cloning vector. These primers are used to amplify the gene of interest by PCR. The target fragment was isolated by gel extraction. Then, the In-Fusion ${ }^{\mathrm{TM}}$ cloning reaction was set up. For a total reaction volume of $10 \mu \mathrm{l}, 2 \mu \mathrm{l}$ of $5 \mathrm{x}$ In-Fusion ${ }^{\mathrm{TM}}$ Reaction Buffer, $1 \mu \mathrm{l}$ In-Fusion ${ }^{\mathrm{TM}}$ Enzyme, a $2: 1$ molar ratio of insert : vector and the appropriate amount of $\mathrm{ddH}_{2} \mathrm{O}$ were used. The cloning reaction was incubated for 15 minutes at $37{ }^{\circ} \mathrm{C}$ followed by additional 15 minutes at $50{ }^{\circ} \mathrm{C}$. After that the reaction volume was brought up to $50 \mu \mathrm{l}$ with TE buffer ( $\mathrm{pH} \mathrm{8)}$ ). Next, the constructs were transformed into either DH5 $\alpha$ or Stella cells (compare 2.2.4.3).

\subsubsection{Preparation of glycerin stocks}

Every construct has to be maintained as a glycerin stock. Glycerin stocks were prepared according to Wieczerzak (2012). Therefore, a single bacterial colony was picked from an LB plate and inoculated in $5 \mathrm{ml} \mathrm{LB}$ medium with $5 \mu \mathrm{l}$ of an appropriate antibiotic $(50 \mathrm{mg} / \mathrm{ml})$. Inoculated samples were incubated overnight at $37{ }^{\circ} \mathrm{C}$ continuously shaking at $120 \mathrm{rpm} .700 \mu \mathrm{l}$ of the bacterial culture was mixed with $300 \mu 150 \%$ glycerin and stored at $-80{ }^{\circ} \mathrm{C}$. 


\subsubsection{PCR}

The PCR is a method where a polymerase is used to exponentially amplify a desired DNA sequence in vitro (Kleppe et al., 1971; Mullis et al., 1986). As a template a double strand DNA is used which is denaturised by heating up to $94{ }^{\circ} \mathrm{C}$. At a lower temperature the primer hybridisation takes place. Primers are short oligonucleotides which are complementary to a specific region of the template and mark the starting point of the DNA synthesis. An increase of the temperature leads to the activation of the polymerase which binds to the primers and synthesises a new DNA strand complementary to the DNA template strand by using deoxynucleotide triphosphates (dNTPs). This cycle is repeated normally 30-35 times.

\section{a) Immolase IM $^{\mathrm{TM}}$}

The Immolase ${ }^{\mathrm{TM}}$ DNA Polymerase (Bioline) is a thermostable DNA polymerase isolated from a novel organism (Bioline, n.d.). This polymerase is characterised by excellent specificity and robust performance and it is able to eliminate primer-dimers or mis-primed products (Bioline, n.d.). In this work the Immolase $^{\mathrm{TM}}$ DNA ploymerase was used to perform genotyping of mice. Furthermore, this DNA polymerase was used to test the oligonucleotides for RT-qPCR and check transcribed cDNA. The PCR reactions were assembled according to the manufacturer's recommendations. Considering the PCR program the activation conditions were optimised.

Table 21: PCR reaction conditions (Immolase ${ }^{\mathrm{TM}}$ )

\begin{tabular}{|c|c|}
\hline component & volume $(\mu \mathrm{l})$ \\
\hline $10 \mathrm{x}$ Immo-Puffer & 2.5 \\
\hline $\mathrm{MgCl}_{2}(50 \mathrm{mM})$ & 0.75 \\
\hline dNTPs (je 2,5 mM) & 0.5 \\
\hline forward primer $(10 \mathrm{pmol} / \mu \mathrm{l})$ & 0.5 \\
\hline reverse primer $(10 \mathrm{pmol} / \mu \mathrm{l})$ & 0.5 \\
\hline IMMOLASE $^{\mathrm{TM}}$ DNA Polymerase $(5 \mathrm{U} / \mu \mathrm{l})$ & 0.2 \\
\hline template DNA & 1 \\
\hline $\mathrm{ddH}_{2} \mathrm{O}$ & to 25 \\
\hline
\end{tabular}


Materials and methods

Table 22: PCR program (Immolase $\left.{ }^{\mathrm{TM}}\right)$

\begin{tabular}{l|c|c|c}
\hline step & temperature $\left({ }^{\circ} \mathbf{C}\right)$ & time & cycle \\
\hline Activation & 94 & $5 \mathrm{~min}$ & 1 \\
\hline Denaturation & 94 & $30 \mathrm{sec}$ & \\
\hline Annealing & $60 / 67$ & $45-60 \mathrm{sec}$ & $30-35$ \\
\hline Extension & 72 & $1 \mathrm{~min}$ & \\
\hline Final extension & 72 & $5-7 \mathrm{~min}$ & 1 \\
\hline Cooling & 4 & $\infty$ & 1 \\
\hline
\end{tabular}

\section{b) peqGOLD Pwo-DNA-Polymerase}

The peqGOLD Pwo-DNA-Polymerase (Peqlab) was originally isolated form the thermophilic archaebacterium Pyrococcus woesei and is thermal stable (Peqlab, n.d.). It works highly processive and has a proof-reading function by $3^{\prime} \rightarrow 5^{\prime}$ exonuclease activity (Peqlab, n.d.). This polymerase was used to amplify products which are used to generate constructs for Co-IP and $\mathrm{Y} 2 \mathrm{H}$ experiments. Based on the $3^{\prime} \rightarrow 5^{\prime}$ exonuclease activity of the Pwo-DNA-Polymerase it is possible that primers and template are destroyed. Therefore, two master mixes are prepared and right before starting the PCR combined according to the company's instructions.

Table 23: PCR reaction conditions (peqGOLD Pwo)

\begin{tabular}{|c|c|}
\hline component & volume $(\mu \mathrm{l})$ \\
\hline \multicolumn{2}{|l|}{ Master mix I (25 $\mu \mathrm{l})$ : } \\
\hline dNTPs (Invitrogen) & 4 \\
\hline forward primer $(10 \mathrm{pmol} / \mu \mathrm{l})$ & 2 \\
\hline reverse primer $(10 \mathrm{pmol} / \mu \mathrm{l})$ & 2 \\
\hline template DNA & 1 \\
\hline $\mathrm{ddH}_{2} \mathrm{O}$ & to 25 \\
\hline \multicolumn{2}{|l|}{ Master mix II (25 $\mu \mathrm{l})$ : } \\
\hline $10 \times$ reaction Buffer complete & 5 \\
\hline Pwo-DNA-Polymerase $(1 \mathrm{U} / \mu \mathrm{l})$ & 3 \\
\hline $\mathrm{ddH}_{2} \mathrm{O}$ & to 25 \\
\hline
\end{tabular}


Materials and methods

Table 24: PCR program (peqGOLD Pwo)

\begin{tabular}{l|c|c|c}
\hline step & temperature $\left({ }^{\circ} \mathbf{C}\right)$ & time & cycle \\
\hline Activation & 94 & $2 \mathrm{~min}$ & 1 \\
\hline Denaturation & 94 & $15 \mathrm{sec}$ & \\
\hline Annealing & $54 / 64$ & $30 \mathrm{sec}$ & 40 \\
\hline Extension & 68 & $2 \mathrm{~min}$ & \\
\hline Final extension & 68 & $7 \mathrm{~min}$ & 1 \\
\hline Cooling & 4 & $\infty$ & 1 \\
\hline
\end{tabular}

c) Pfu Ultra ${ }^{\text {TM }}$ High-Fidelity DNA Polymerase

The Pfu polymerase is found in the hyperthermophilic archaeon Pyrococcus furiosus. PfuUltra ${ }^{\mathrm{TM}}$ High-Fidelity DNA Polymerase is formulated with the ArchaeMaxx ${ }^{\circledR}$ polymerase enhancing factor, resulting in improved overall PCR performance (AgilentTechnologies, n.d.). The PfuUltra ${ }^{\mathrm{TM}}$ High-Fidelity DNA Polymerase (Stratagene) was used to amplify products for engineering constructs for Y2H. PCR reaction conditions recommended by the company were optimised by adding $1 \mu \mathrm{l}$ of $\mathrm{MgSO}_{4}$ and modifying the concentration of dNTPs and the polymerase. The annealing and the denaturation cycle were extended from 30 seconds to 45 seconds and the temperature of the extension cycles were set at $72{ }^{\circ} \mathrm{C}$ instead of $68{ }^{\circ} \mathrm{C}$. 
Table 25: PCR reaction conditions (PfuUltra ${ }^{\text {TM }}$ High-Fidelity)

\begin{tabular}{l|l}
\hline component & volume $(\boldsymbol{\mu l})$ \\
\hline $10 \mathrm{x} P f u$ Ultra HF reaction buffer & 2.5 \\
\hline $\mathrm{MgSO}_{4}(50 \mathrm{mM})$ & 1 \\
\hline $\mathrm{dNTPs}(2,5 \mathrm{mM}$ each $)$ & 0.8 \\
\hline forward primer $(10 \mathrm{pmol} / \mu \mathrm{l})$ & 0.5 \\
\hline reverse primer $(10 \mathrm{pmol} / \mu \mathrm{l})$ & 0.5 \\
\hline$P f u \mathrm{Ultra}{ }^{\mathrm{TM}}$ high-fidelity DNA polymerase $(5 \mathrm{U} / \mu \mathrm{l})$ & 1 \\
\hline template DNA $^{-}$ & 1 \\
\hline $\mathrm{ddH}_{2} \mathrm{O}$ & to 25 \\
\hline
\end{tabular}

Table 26: PCR program (PfuUltra ${ }^{\mathrm{TM}}$ High-Fidelity)

\begin{tabular}{l|c|c|c}
\hline step & temperature $\left({ }^{\circ} \mathbf{C}\right)$ & time & cycle \\
\cline { 1 - 3 } Activation & 95 & $2 \mathrm{~min}$ & 1 \\
\hline Denaturation & 95 & $45 \mathrm{sec}$ & \\
\hline Annealing & 60 & $45 \mathrm{sec}$ & 35 \\
\hline Extension & 68 & $2 \mathrm{~min}$ & \\
\hline Final extension & 68 & $10 \mathrm{~min}$ & \multirow{2}{*}{1} \\
\hline Cooling & 4 & $\infty$ & 1 \\
\hline
\end{tabular}

\section{d) Platinum ${ }^{\circledR}$ Taq DNA polymerase}

The Platinum ${ }^{\circledR}$ Taq DNA Polymerase is found in the thermophilic bacterium Thermus aquaticus and it has a $5^{\prime} \rightarrow 3^{\prime}$ polymerase and a $5^{\prime} \rightarrow 3^{\prime}$ exonuclease activity (Invitrogen, 2010). The polymerase is complexed with a proprietary antibody that blocks polymerase activity at low temperatures (Invitrogen, 2010). A "hotstart" at $94{ }^{\circ} \mathrm{C}$ restores the activity (Invitrogen, 2010). In this work the Platinum ${ }^{\circledR}$ Taq DNA Polymerase (Invitrogen) was also used to amplify products for engineering constructs for Y2H. Furthermore, it was applied to distinguish the gender of mouse embryos. The PCR reaction was set up according the company's recommendations. 
The activation, denaturation and annealing cycles were optimised and a final extension step included.

Table 27: PCR reaction conditions (Platinum ${ }^{\circledR}$ Taq)

\begin{tabular}{l|l}
\hline component & volume $(\mu \mathrm{l})$ \\
\hline $10 \mathrm{x}$ PCR buffer & 2.5 \\
\hline $\mathrm{MgCl}_{2}(50 \mathrm{mM})$ & 0.75 \\
\hline $\mathrm{dNTPs}(2,5 \mathrm{mM}$ each $)$ & 0.5 \\
\hline forward primer $(10 \mathrm{pmol} / \mu \mathrm{l})$ & 0.5 \\
\hline reverse primer $(10 \mathrm{pmol} / \mu \mathrm{l})$ & 0.5 \\
\hline Platinum ${ }^{\circledR}$ Taq $\mathrm{DNA}$ polymerase $(5 \mathrm{U} / \mu \mathrm{l})$ & 0.2 \\
\hline template DNA & 1 \\
\hline $\mathrm{ddH}_{2} \mathrm{O}$ & to 25 \\
\hline
\end{tabular}

Table 28: PCR grogram (Platinum ${ }^{\circledR}$ Taq)

\begin{tabular}{l|c|c|c}
\hline step & temperature $\left({ }^{\circ} \mathbf{C}\right)$ & time & cycle \\
\hline Activation & $94 / 95$ & $5 \mathrm{~min}$ & 1 \\
\hline Denaturation & $94 / 95$ & $45 \mathrm{sec}$ & \\
\hline Annealing & $59 / 61$ & $45 \mathrm{sec}$ & $35 / 40$ \\
\hline Extension & 72 & $1.5 / 2 \mathrm{~min}$ & \\
\hline Final extension & 72 & $5 / 7 \mathrm{~min}$ & 1 \\
\hline Cooling & 4 & $\infty$ & 1 \\
\hline
\end{tabular}

\subsubsection{Touchdown PCR}

The touchdown PCR is a method to avoid amplifying nonspecific sequences by unspecific primer binding. Different annealing temperatures are used starting with high temperatures. The upper limit of the annealing temperature is set by the melting point of the primers used. The increments of annealing temperature decrease as well as the number of cycles can be chosen individually. At temperatures just below the melting point the annealing of primers to the template is most specific while at lower temperatures primers bind less specifically. By the exponential nature of the polymerase amplification the specific sequences amplified in early steps will 
overwhelm the nonspecific sequences amplified later (Korbie and Mattick, 2008). For the touchdown PCR applied for mutation screens with human samples the Immolase ${ }^{\mathrm{TM}}$ DNA polymerase was used.

Table 29: PCR reaction conditions (Touchdown)

\begin{tabular}{|c|c|}
\hline component & volume $(\mu \mathrm{l})$ \\
\hline 10 x Immo-Puffer & 2.5 \\
\hline $\mathrm{MgCl}_{2}(50 \mathrm{mM})$ & 0.75 \\
\hline dNTPs (2,5 mM each) & 0.5 \\
\hline forward primer $(10 \mathrm{pmol} / \mu \mathrm{l})$ & 0.65 \\
\hline reverse primer $(10 \mathrm{pmol} / \mu \mathrm{l})$ & 0.65 \\
\hline IMMOLASE $^{\mathrm{TM}}$ DNA Polymerase $(5 \mathrm{U} / \mu \mathrm{l})$ & 0.2 \\
\hline template DNA & 1 \\
\hline $\mathrm{ddH}_{2} \mathrm{O}$ & to 25 \\
\hline
\end{tabular}

Table 30: PCR program (Touchdown)

\begin{tabular}{l|c|c|c}
\hline step & temperature $\left({ }^{\circ} \mathbf{C}\right)$ & time & cycle \\
\hline Activation & 95 & $7 \mathrm{~min}$ & 1 \\
\hline Denaturation & 95 & $30 \mathrm{sec}$ & \\
\hline Annealing & $65-1$ until 60 & $1 \mathrm{~min}$ & 5 \\
\hline Extension & 72 & $1 \mathrm{~min}$ & \\
\hline Denaturation & 95 & $30 \mathrm{sec}$ & 30 \\
\hline Extension & 72 & $1 \mathrm{~min}$ & \\
\hline Final extension & 72 & $5 \mathrm{~min}$ & 1 \\
\hline Cooling & 4 & $\infty$ & \\
\hline
\end{tabular}

\subsubsection{Sequencing PCR}

The sequence analysis used in this work is modified from the chain determination method by Sanger and Coulson (Sanger and Coulson, 1975). This variation of a PCR uses only one primer. Besides from all four standard deoxynucleotide triphosphates (dNTPs) modified dideoxynucleotide triphosphates (ddNTPs) are used. The four ddNTPs are differently labeled with fluorescent dye and lack a 3'-hydroxy (OH-) 
group that is required for the formation of a phosphodiester bond between two nucleotides which causes termination of DNA strand elongation (Atkinson et al., 1969). The consequences are DNA fragments of different lengths that are separated by size using a capillary electrophorese (MegaBACE ${ }^{\mathrm{TM}} 1000$, Amersham). Stimulated by laser the fluorescently labeled ddNTPs emit light at different wavelengths. The sequence is visualised as peaks of different height and shape (chromatogram).

Table 31: PCR reaction conditions (sequencing)

\begin{tabular}{l|l}
\hline component & volume $(\boldsymbol{\mu l})$ \\
\hline $5 \times$ sequencing buffer & 2 \\
\hline BigDye $^{\circledR}$ & 1 \\
\hline forward primer or reverse primer $(10 \mathrm{pmol} / \mu \mathrm{l})^{\text {template DNA }}$ & 1 \\
\hline $\mathrm{ddH}_{2} \mathrm{O}$ & $1-6$ \\
\hline
\end{tabular}

Table 32: PCR program (sequencing)

\begin{tabular}{l|c|c|c}
\hline step & temperature $\left({ }^{\circ} \mathbf{C}\right)$ & time & cycle \\
\hline Activation & 95 & $1 \mathrm{~min}$ & 1 \\
\hline Denaturation & 95 & $30 \mathrm{sec}$ & \\
\hline Annealing/Extension & 60 & $2.5 \mathrm{~min}$ & 30 \\
\hline Final extension & 60 & $5 \mathrm{~min}$ & 1 \\
\hline Cooling & 8 & $\infty$ & 1 \\
\hline
\end{tabular}

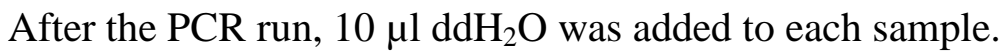

\subsubsection{Quantitative real-time PCR and data analysis}

The RT-qPCR is used to amplify and simultaneously quantify cDNA samples reverse transcribed from mRNA (messenger RNA). Therefore, SYBR Green I, an asymmetrical cyanine dye that intercalates with double stranded DNA is used (Zipper et al., 2004). In this complex SYBR Green I absorbs blue light. The excitation maximum is about $490 \mathrm{~nm}$ and it has an emission maximum of approximately 
$520 \mathrm{~nm}$. This fluorescent signal can be detected by a detection system during qPCR (quantitative real-time PCR) and is proportional to the amplified DNA.

Absolute and relative quantification of nucleic acids are possible by using this method. In this work the relative quantification of gene expression was used. Thereby, an internal reference gene (housekeeping gene) is needed to determine the fold change in expression. ROX (6-Carboxyl-X-Rho-damine) is used as a passive reference dye to normalise the fluorescence signal of SYBR Green I (Illumina, 2011). Unspecific influences such as variations caused by pipetting errors can be compensated.

For the RT-qPCR RNA of mouse embryos were reverse transcribed into cDNA. Serial 1:5 dilutions of the cDNA were prepared for each analysed gene which should be analysed to determine the standard curve. To exclude contamination, $\mathrm{ddH}_{2} \mathrm{O}$ was used as a blank value for each gene. A triple measurement was performed for all analysed genes, respectively. Samples were applied onto a 384 well plate and analysed with “ABI PRISM ${ }^{\circledR} 7900$ HT Sequence Detection System”. Below the RT$\mathrm{qPCR} / \mathrm{qPCR}$ program and the reaction conditions are listed.

Table 33: RT-qPCR/qPCR program

\begin{tabular}{l|c|c|c}
\hline step & temperature $\left({ }^{\circ} \mathbf{C}\right)$ & time & cycle \\
\hline \multirow{2}{*}{ Activation } & 50 & $2 \mathrm{~min}$ & 1 \\
\hline Denaturation & 95 & $15 \mathrm{~min}$ & 1 \\
\hline Annealing & 94 & $15 \mathrm{sec}$ & 40 \\
\hline Extension & 60 & $30 \mathrm{sec}$ & \\
\hline \multirow{2}{*}{ Dissociation } & 72 & $1 \mathrm{~min}$ & \\
\hline
\end{tabular}


Table 34: reaction conditions (RT-qPCR/qPCR)

\begin{tabular}{l|l}
\hline component & volume $(\boldsymbol{\mu l})$ \\
\hline SYBR Green I & 5 \\
\hline cDNA or human genomic DNA & 2.5 \\
\hline forward primer $(1 \mathrm{pmol} / \mu \mathrm{l})$ & 1.25 \\
\hline reverse primer $(1 \mathrm{pmol} / \mu \mathrm{l})$ & 1.25 \\
\hline
\end{tabular}

The raw data were evaluated using the sequence-detection software (SDS Version 2.1, PE Applied Biosystems). The SDS software displays the results of the RTqPCR/qPCR run and plots them in a graph of normalised reporter fluorescence $\left(R_{n}\right)$ versus cycle number (Fig. 4). In table 35 is the definition of terms listed which are used in quantification analysis.

Table 35: Terms used in Allelic Discrimination Analysis.

\begin{tabular}{l|l}
\hline \multicolumn{1}{c|}{ Term } & \multicolumn{1}{c}{ Definition } \\
\hline Baseline & $\begin{array}{l}\text { A line fit to fluorescence intensity values during } \\
\text { the initial cycles of PCR, in which there is little } \\
\text { change in fluorescence signal. }\end{array}$ \\
\hline Threshold cycle $\left(C_{T}\right)$ & $\begin{array}{l}\text { The fractional cycle number at which the } \\
\text { fluorescence intensity exceeds the threshold } \\
\text { intensity. }\end{array}$ \\
\hline Passive reference & $\begin{array}{l}\text { A dye that provides an internal fluorescence } \\
\text { reference to which the reporter dye signal can be } \\
\text { normalized during data analysis. Normalization is } \\
\text { necessary to correct for fluorescence fluctuations } \\
\text { caused by changes in concentration or volume. }\end{array}$ \\
\hline Reporter dye & $\begin{array}{l}\text { The dye (SYBR Green } I) \text { provides a signal that } \\
\text { indicates specific amplification. }\end{array}$ \\
\hline Delta $\mathbf{R n}(\Delta \mathbf{R n})$ & $\begin{array}{l}\text { The ratio of the fluorescence intensity of the } \\
\text { reporter dye signal to the fluorescence intensity of } \\
\text { the passive reference dye signal. }\end{array}$ \\
\hline
\end{tabular}

Table modified after AppliedBiosystems (n.d.). 


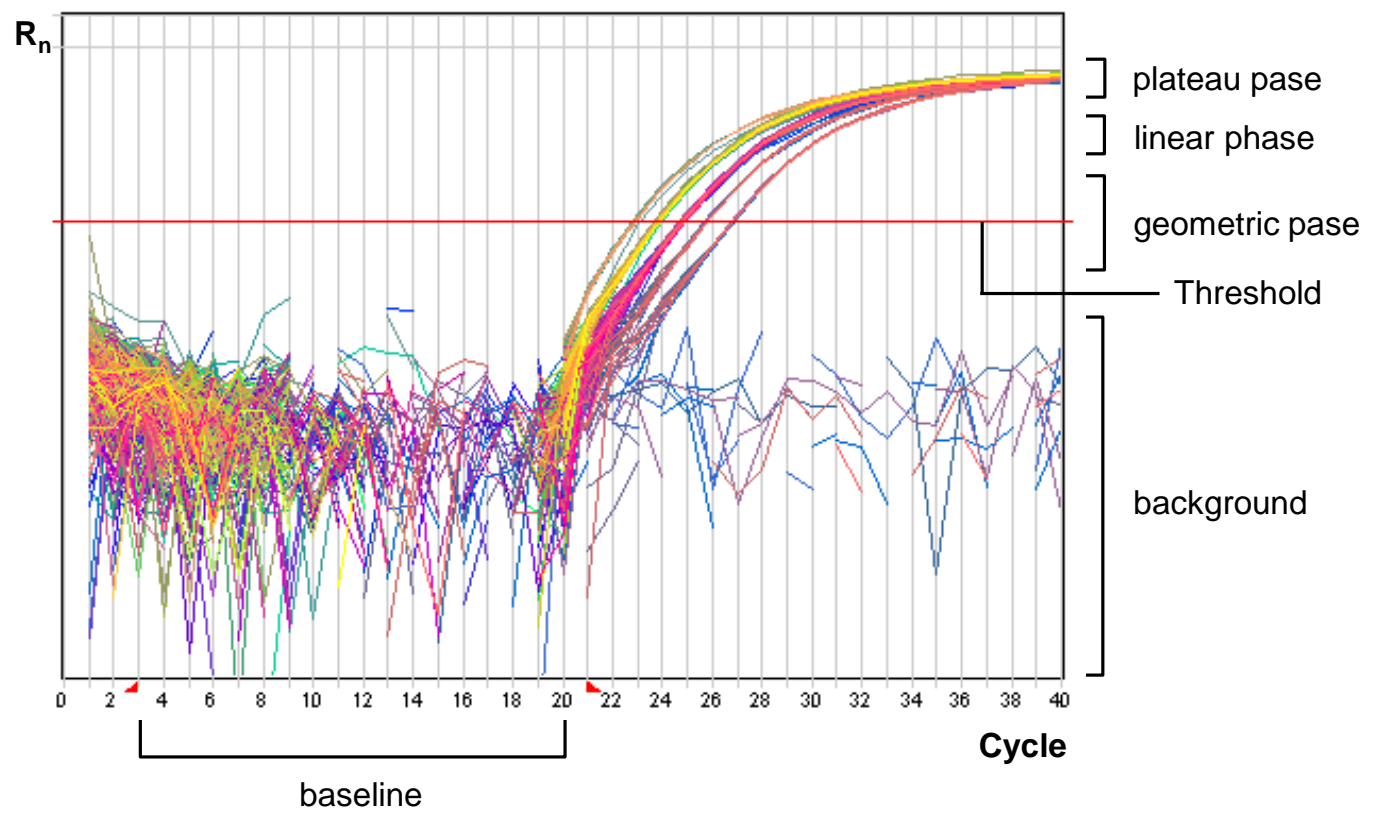

Figure 4: Results after RT-qPCR/qPCR run.

The graph shows the normalised reporter fluorescence $(\mathrm{Rn})$ (ordinate) versus cycle number (abscissa). The threshold is set at a certain $\mathrm{Rn}$ value during the exponential amplification phase (geometric phase) slightly above background. Within this phase optimal PCR conditions exists, whereas in the linear and plateau phase the PCR reaction is inhibited due to limited availability of substrates or starting destruction of the polymerase or nucleotides. The number of cycles at which the fluorescence intensity exceeds the threshold intensity is called the threshold cycle $(\mathrm{Ct})$. With the $\mathrm{Ct}$ values the quantification of the DNA samples can be determined. Figure modified after AppliedBiosystems (n.d.).

Data were exported to Microsoft Excel and further processed according to Dr. Arne Zibat's instructions. By using the serial 1:5 dilution of the DNA template a standard curve for each target gene was determined. Therefore, the initial concentration of the template DNA has to be known. By plotting the logarithms of the initial concentrations (ordinate) versus the median Ct values (abscissa) the linear equation $y=m x+b$ were achieved for the computation of a standard curve. The constant $m$ is the slope of the straight line, constant $b$ indicates were the straight line intercepts the $\mathrm{y}$-axis and $\mathrm{x}$ is the independent variable. Next, the efficiency (E) was determined: $\mathrm{E}$ $=10^{(-1 / \mathrm{m})}-1$. A slope $(\mathrm{m})$ of -3.32 results in an efficiency of $100 \%$. To analyse the data, the efficiency should be between $85 \%$ and $105 \%$. Values above $100 \%$ indicate an inhibition of the $\mathrm{qPCR} . \mathrm{R}^{2}$ displays the scattering around the straight line and indicates the reliability of the data. $\mathrm{R}^{2}$ should be at the minimum of $95 \%$. Next, the target genes were normalised against three reference genes (housekeeping genes) to compensate variations during qPCR. An ideal control should be easy to detect and 
its expression should be stable in different cell types, during the cell cycle or during certain outer influences (Bustin et al., 2009). For the normalisation the logarithms of the initial concentrations of the reference genes were determined by using the formula: $\log$ (initial concentration) $=(\mathrm{Ct}-\mathrm{b}) / \mathrm{m}$. Next, the logarithms were countermanded and the mean as well as the standard deviation of the initial concentration were determined. Now, the target genes were normalised against the three reference genes. Therefore, the logarithms of the initial concentrations of the target genes were determined and the logarithms countermanded as for the reference genes before. After that, the initial concentration were used for the normalisation $(\mathrm{N}): \mathrm{N}=$ initial concentration/mean of the initial concentration of the reference genes. The mean and the standard deviation of the normalisation were determined. Further, the relative change in expression of the target genes was compared to the calibrator (pooled wild-type samples): N/mean of the initial concentration of the calibrator. The mean and the standard deviation of the relative change in expression were determined. Finally, the mean of the relative change in expression of each target gene against the three reference genes were determined and results were represented in a diagram.

For the patient screen to check for duplications and deletions within exons of certain genes, qPCR was performed under adherence of diagnostic standards. Based on the determined concentrations of the DNA samples, patient probes and the wild-type control were brought to a concentration of $1.25 \mathrm{ng} / \mu \mathrm{l}$ using $\mathrm{ddH}_{2} \mathrm{O}$. Using standard DNA (Human DNA Male, ABI) four different concentrations $(2.5 \mathrm{ng} / \mu 1,1.25 \mathrm{ng} / \mu \mathrm{l}$, $0625 \mathrm{ng} / \mu \mathrm{l}$ and $0.3125 \mathrm{ng} / \mu \mathrm{l})$ were adjusted. Two reference genes were used and for each analysed probe a no template control (NTC) (DNA volume was replaced by the same volume of $\mathrm{ddH}_{2} \mathrm{O}$ ) was carried along to check for contaminations. For each probe a double parallel measurement was performed.

A total reaction volume of $10 \mu \mathrm{l}$ (Tab. 34) was supplied on a 384 well plate. First, $2.5 \mu \mathrm{l}$ of primer-mix were placed into the wells of a 384 well plate which was then centrifuged for 2 minutes at 1,500 rpm. Next, $7.5 \mu 1$ of a mixture containing the DNA probe and SYBR Green I was added to the wells. To eliminate air bubbles, the plate was centrifuged for 10 minutes at 3,000 rpm and then placed into the "ABI PRISM $^{\text {TM }} 7900$ HT Fast Real-Time PCR System" to record the data.

The data were analysed using the software SDS2.3 according to the operating instructions (2012) of the diagnostic department of the Human Genetic Institute, 
Goettingen. First, the data were checked for contaminations by using the NTCs. Clear signals indicate a possible contamination and the analysis of this probe has to be repeated. Next, the dissociation curve should show a single peak, which indicates a unified product. If double peaks are present (unspecific side product), the analysis has to be repeated. For each primer pair a standard curve and $\mathrm{R}^{2}$ value were determined by the SDS software. For $\mathrm{R}^{2}$, values should be between 0.99 and 0.98 .

Next, data were exported and analysed using Microsoft Excel. The multiple of the median $(\mathrm{MOM})$ was determined: $\mathrm{MOM}=$ quantity/mean quantity of all references. For each double measured probe the median of the MOM and the standard deviation were determined and the values rounded to two decimal places after the decimal point. The mean MOM values were presented for each analysed probe in a column diagram. A deletion reveals in the calculation a theoretical value of MOM 0.4-0.6 and duplication a theoretical value of MOM 1.4. The theoretical value of MOM for wild-type is 1.0. Since the qPCR is a sensitive method, the determined values are subjected to variation, so they are often present below or above these theoretical values. Therefore, values below MOM 0.8 and above MOM 1.2 are classified to be suspicious. The reactions have to be repeated.

\subsubsection{Purification of DNA}

The MSB ${ }^{\circledR}$ Spin PCRapace Kit (Invitek) was used for fast and efficient direct purification of PCR products, digested samples and mini preparations according to the manufacturer's instructions with an additional centrifugation step. All centrifugation steps were carried out at room temperature. Up to $50 \mu 1$ PCR samples $250 \mu \mathrm{l}$ Binding Buffer was added to the PCR mixture. The sample was mixed vigorously by pipetting, then transferred onto a Spin Filter to bind the PCR fragments. Next, samples were centrifuged at 12,000 rpm for 3 minutes and the flowthrough was discarded. An additional centrifugation step at 13,000 rpm for 1 minute was performed to fully remove all ethanol. The flow-through was discarded and the Spin Filter was placed into a new tube. To elute the PCR fragments, at least $10 \mu 1$ $\mathrm{ddH}_{2} \mathrm{O}$ was supplied directly onto the center of the Spin Filter. After 1 minute incubation at room temperature, the samples were centrifuged at 10,000 rpm for 1 minute. Purified samples were stored at $-20^{\circ} \mathrm{C}$. 


\subsubsection{Agarose gel electrophoresis}

To separate DNA fragments according to their length after PCR amplification, restriction digestion and ligation reaction, standard agarose gel elelectrophoresis was used (Fisher and Dingman, 1971; Helling et al., 1974). Depending on the DNA fragment length, 0.5-2\% agarose gels were prepared using agarose (Bio-Budget) and $1 \mathrm{x}$ Tris/Acetate/EDTA (TAE) buffer or $0.5 \mathrm{x}$ Tris/Borate/EDTA (TBE) buffer (Tab. 7). The mixture was boiled in the microwave until the agarose was completely dissolved. As running buffer $1 \mathrm{x}$ TAE buffer or $0.5 \mathrm{x}$ TBE buffer were used. To visualise the DNA under UV light, one drop of Ethidium bromide (AppliChem) (Sharp et al., 1973) or Roti Safe (Roth) was added to the agarose mixture. Gels were placed in horizontal gel chambers (Owl Scientific). Gels were loaded according to Lee et al. (2012).

\subsubsection{Gel extraction}

The QIAquick Gel Extraction Kit (Qiagen) is used to extract and purify DNA (70 bp$10 \mathrm{~kb}$ ) from agarose gels in TAE or TBE buffer according to the manufacturer's instructions. All centrifugation steps were carried out at $13,000 \mathrm{rpm}$ at room temperature. The desired DNA fragment was excised from the agarose gel using a clean scalpel. If possible, extra agarose was removed before the gel slice was scaled in a colourless tube. To 1 volume of gel ( $1 \mathrm{mg}$ is equivalent to $100 \mu \mathrm{l}) 3$ volumes of Buffer QG were added to the gel slice. In case of $>2 \%$ agarose gels, 6 volumes Buffer QG were needed. Next, the tube with the gel slice was incubated 10 minutes at $50{ }^{\circ} \mathrm{C}$ until the gel slice was completely dissolved. Every 2-3 minutes the tubes were vortexed during incubation to support dissolution. Since the adsorption of DNA to the QIAquick membrane is efficient only at $\mathrm{pH} \leq 7.5$, it is necessary that the colour of the mixture stays yellow indicating the required $\mathrm{pH}$. In case the mixture is orange or violet after the gel has dissolved, $10 \mu \mathrm{l}$ of $3 \mathrm{M}$ sodium acetate (pH 5) had to be added. To DNA fragments $<500 \mathrm{bp}$ and $>4 \mathrm{~kb} 1$ gel volume of isopropanol was added which increases the yield of DNA fragments. Next, a QIAquick spin column was placed in the provided $2 \mathrm{ml}$ collection tube before the sample was applied to the column to bind DNA. The probes were centrifuged for 1 minute. The column reservoir is $800 \mu \mathrm{l}$. For sample volumes $>800 \mu \mathrm{l}$, the column was simply loaded and centrifuged again. The flow-through was discarded and the column 
placed back in the same collection tube. $500 \mu \mathrm{l}$ Buffer QG was added to the column and centrifuged for 1 minute. The flow-throw was discarded and $750 \mu 1$ of Buffer PE was added to the column for washing. After 2-5 minutes incubation, the column was centrifuged for 1 minute. The flow-through was removed and the column centrifuged again for 1 minute to completely remove the ethanol from the Buffer PE. To elute the

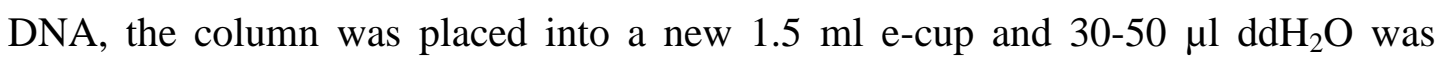
added to the center of the QIAquick membrane. After 1 minute incubation the column was centrifuged for 1 minute. The DNA samples were stored at $-20{ }^{\circ} \mathrm{C}$

\subsubsection{Mouse preparation}

One-to-one matings of heterozygous Whirligig $\left(C h d 7^{W h i /+}\right)$ females and males were set up. To determine the age of the embryos, a daily check for a vaginal plug (VP check) was performed on female Whirligig mice. The day when the copulation plug was observed, was counted as embryonic stage 0.5 (E0.5). At the required day the pregnant Whirligig mice were sacrificed by cervical dislocation and the uterus was removed. The embryos were dissected from the yolk sacs and washed with PBS. To determine the gender of the embryos, a tail biopsy was performed. For protein and RNA isolation the embryos were flash frozen on liquid nitrogen and stored at $-80^{\circ} \mathrm{C}$. For paraffin sections the embryos were fixed overnight at $4{ }^{\circ} \mathrm{C}$ in $4 \%$ paraformaldehyde.

\subsubsection{Fixation and dehydration of embryos for paraffin embedding}

Fixation and dehydration of mouse embryos have been carried out according to Luxan et al. (2013) with some modifications, such as additional incubation steps and longer incubation times. Mouse embryos isolated from the uterus were fixated overnight in $4 \%$ paraformaldehyde in PBS at $4{ }^{\circ} \mathrm{C}$. The next day embryos were washed for 10 minutes in PBS. If not indicated differently, all steps were carried out at room temperature. By an increasing ethanol row $(50 \%, 70 \%, 80 \%, 90 \%$ ethanol/PBS and $100 \%$ ethanol) embryos were dehydrated. In contrast to the procedure performed by Luxan et al. (2013) an additional $50 \%$ ethanol/PBS incubation step was carried out and each concentration step was performed twice for 1 hour. After the dehydration, embryos were incubated overnight instead of 30 minutes in $100 \%$ xylene. Additionally, embryos were incubated in $50 \%$ 
paraffin/xylene for 2-6 hours at $65^{\circ} \mathrm{C}$. Embryos were transferred into $100 \%$ paraffin and incubated for 2 days instead of 3 hours at $65^{\circ} \mathrm{C}$. During that time the paraffin was exchanged at least six times. The embryos were ready to be placed into forms to make paraffin blocks for the preparation of sections.

\subsubsection{Preparation of paraffin sections of mouse embryos}

To prepare paraffin sections, the microtome (JUNG RM 2035, Leica) were used. First, the paraffin block with the fixed embryo was cut to the size needed and clamped onto the microtome. Sections of 6-8 $\mu \mathrm{m}$ thickness were made which were carefully transferred to the surface of water $\left(58^{\circ} \mathrm{C}\right)$ from where they were placed on slides (Thermo Scientific). After the sections were dried, they were stored at $4{ }^{\circ} \mathrm{C}$ or room temperature until needed.

\subsubsection{Microarray and data analysis}

Microarrays are used to measure the expression of a large number of genes (Schena et al., 1995). In this work the microarrays were performed by the TAL in Goettingen as described by Schulz et al. (2014b) using the "Low RNA Input linear Amplification Kit Plus, One Color" protocol (Agilent Technologies, Inc. 2007; Cat. N: 5188-5339) and the RNA Spike-In Kit for One color (Agilent Technologies, Inc. 2007; Cat. № 5188-5282) following the manufacturer's instructions. For the analysis of global gene expression the Mice 4 x $44 \mathrm{~K}$ design array from Agilent Technologies (G4846A) was used following the manufacturer's standard protocol. $200 \mathrm{ng}$ of total RNA was used for reverse transcription into cDNA following the company's recommendation. The in vitro transcription was done according to the manufacturer's instructions. To determine the quantity and efficiency of the amplified cRNA that was labeled, the NanoDrop ND-1000 UV-VIS Spectrophotometer version 3.2.1 was used. The hybridisation procedure took place in the Hybridisation Oven (Agilent). Probes were incubated for 17 hours at $10 \mathrm{rpm}$ at $65^{\circ} \mathrm{C}$. The following washing and staining were performed according to the manufacturer's standard protocol. All together the microarray data were generated according to the Minimum Information About a Microarray Experiment (MIAME) guidelines (compare NCBI's Gene Expression Omnibus (GSE46591). The Agilent DNA microarray scanner (G2505B) was used at 5 micron resolution to detect the $\mathrm{Cy} 3$ intensities by one-color scanning. Next, the 
scanned image files were screened for artefacts and further analysed conforming to Opitz et al. (2010). The Feature Extraction Software Version was changed to 10.7.3.1. DAVID was used to find over-represented functions (Huang da et al., 2009b, a)

\subsubsection{Cell biological methods}

\subsubsection{Culturing of eukaryotic cells}

In this work human HeLa cells derived from cervical cancer cells were used (Scherer et al., 1953). The adherent cells were cultured in $25 \mathrm{~cm}^{2}$ and $75 \mathrm{~cm}^{2}$ culture flasks (Sarstedt) in $5 \mathrm{ml}$ and $10 \mathrm{ml}$ medium, respectively. The medium consists of Dulbecco's modified Eagle's media (DMEM) (PAN BIOTECH), $10 \%$ foetal calf serum (PAN BIOTECH) and $1 \%$ penicillin/streptomycin (PAN BIOTECH). Cells were cultured at $37{ }^{\circ} \mathrm{C}$ at a humidity of $98 \%$ and $\mathrm{CO}_{2}$ content of $5 \%$. Every two days the medium was exchanged.

To split cells, TrypLE Express (Invitrogen) was used to detach cells from the culture flask. First, cells cultured in a $25 \mathrm{~cm}^{2}$ flask were washed once or twice with $5 \mathrm{ml}$ DPBS. DPBS was removed completely before adding $1.5 \mathrm{ml}$ TrypLE Express. Cells were incubated at $37{ }^{\circ} \mathrm{C}$ for 3 minutes. Then, $3.5 \mathrm{ml}$ medium was added and cells were resuspended and transferred to a $13 \mathrm{ml}$ centrifugation tube and centrifuged at $1,000 \mathrm{rpm}$ for 5 minutes at room temperature. Supernatant was removed and the cell pellet was washed by resuspending in $5 \mathrm{ml}$ DPBS. Tubes were centrifuged again at 1,000 rpm for 5 minutes at room temperature. The supernatant was discarded and the cell pellet was resuspended in medium. A few drops were added to a new $25 \mathrm{~cm}^{2}$ culturing flask with $5 \mathrm{ml}$ fresh medium. Cells were equally distributed and incubated at $37^{\circ} \mathrm{C}$.

\subsubsection{Cryopreservation and revitalisation of eukaryotic cells}

For a permanently storage, cells were cultured in $75 \mathrm{~cm}^{2}$ flask (Sarstedt) until they reached a confluence of $80 \%$. They were washed with DPBS and then trypsinised (2.2.16.1). Cells were resuspended in $1.5 \mathrm{ml}$ DMEM, and $1.5 \mathrm{ml}$ of the Cryo-medium consisting of $50 \%$ FKS, $30 \%$ DMEM and $20 \%$ DMSO was added. Cell mixture was transferred in Cryo-tubes and temporarily incubated at $-20{ }^{\circ} \mathrm{C}$. Then, cells were kept at $-80{ }^{\circ} \mathrm{C}$ or liquid nitrogen. For revitalisation, cells were quickly unfrozen at 
room temperature and resuspended in $9 \mathrm{ml}$ prewarmed culturing medium. Cells were pelleted twice, washed with culturing medium and cultured in a $25 \mathrm{~cm}^{2}$ flask (Sarstedt) with fresh medium (2.2.16.1). After 24 hours the medium was exchanged.

\subsubsection{Transfection of eukaryotic cells with plasmids}

Transfection is the introduction of nucleic acids (DNA or RNA) into cells. In this work HeLa cells were transfected with plasmids containing either a complete gene sequence or certain segments of a gene sequence to overexpress the appropriate DNA sequence in vivo. As transfection reagent lipofectamine 2000 (Invitrogen) was used according to the manufacturer's instructions. Lipofectamine reagent forms cationic liposomes in an aqueous environment which entrap the negatively charged transfection material by forming complexes (Dalby et al., 2004). The cationic lipid molecules are formulated with a neutral lipid that ensures the introduction of the transfection material into the cells (Dalby et al., 2004). HeLa cells were cultured 24 hours before transfection in $75 \mathrm{~cm}^{2}$ flasks (Sarstedt) so they have a confluence of $70-90 \%$ at the time of transfection. $30 \mu \mathrm{g}$ of plasmid DNA was mixed with $1.875 \mathrm{ml}$ Opti MEM (Invitrogen) and incubated for 5 minutes at room temperature. $75 \mu 1$ lipofectamine 2000 was also mixed with $1.875 \mathrm{ml}$ Opti MEM and incubated for 5 minutes at room temperature. The plasmid DNA and the the lipofectamine mixture were combined and incubated for 25 minutes at room temperature. In the meanwhile cells were washed with DPBS and 9 ml DMEM with 1 x NEAA (Gibco) was added. The transfection mixture was added to the cells trop by trop and then incubated at $37^{\circ} \mathrm{C}$. After 4-5 hours, $9 \mathrm{ml}$ DMEM with $20 \%$ FKS and $1 \times$ NEAA was added to the medium. After transfection, cells were incubated at $37^{\circ} \mathrm{C}$ for 24 hours total.

\subsubsection{Protein chemical methods}

\subsubsection{Protein isolation from HeLa cells}

Nuclear proteins from HeLa cells were specifically isolated using the NE-PER Nuclear and Cytoplasmic Extraction Reagents (Thermo Scientific) according to the manufacturer's instruction. First, the medium were removed and cells were washed twice with DPBS and then harvested using TrypLE Express (Invitrogen). To neutralise the trypsin, DMEM medium was added instead of DPBS and the cell suspension was centrifuged at $500 \times g$ for 5 minutes. The supernatant was removed 
and the cell pellet washed by suspending in DPBS. The cell suspension was centrifuged again at $500 \mathrm{x} g$ for 5 minutes. The supernatant was discarded and the cell pellet suspended in $1.5 \mathrm{ml}$ DPBS and then transferred into a $1.5 \mathrm{ml}$ e-cup. Following centrifugation steps were performed at $4{ }^{\circ} \mathrm{C}$, cell samples and extracts were kept on ice. Next, the cells were centrifuged at $500 \times g$ for 2-3 minutes. The supernatant was carefully removed to leave the cell pellet as dry as possible. Depending on the cell volume the reagent volume was determined as shown below.

Table 36: Reagent volumes for different packed cell volume

\begin{tabular}{|c|c|c|c|}
\hline Packed Cell Volume $(\boldsymbol{\mu l})$ & CER I $(\boldsymbol{\mu l})$ & CER II $(\boldsymbol{\mu l})$ & NER $(\boldsymbol{\mu l})$ \\
\hline 10 & 100 & 5.5 & 50 \\
\hline 20 & 200 & 11 & 100 \\
\hline 50 & 500 & 27.5 & 250 \\
\hline 100 & 1,000 & 55 & 500 \\
\hline
\end{tabular}

Right before using CER I and NER protease inhibitors (100 x stock) were added to maintain extract integrity and function. First, ice-cold CER I was added to the cell pellet which was then fully suspended by vortexing on the highest setting for 15 seconds. After 10 minutes incubation, the appropriate amount on ice-cold CER II was added. The tube was vortexed for 5 seconds on the highest setting, then incubated for 10 minutes and vortexed again for 5 seconds on the highest setting. Samples were centrifuged for 5 minutes at maximum speed $(\sim 16,000 \times g)$. The supernatant consisting of the cytoplasmic extract was transferred to a new prechilled tube. To the pellet which contains the nuclei, the appropriate amount on ice-cold NER was added. For a total of 40 minutes the tube was vortexed every 10 minutes for 15 seconds on the highest setting and then centrifuged for 10 minutes at maximum speed $(\sim 16,000 \times g)$. The supernatant which contains the nuclear extract was transferred to a new prechilled tube. The protein concentration was determined by Synergy Mx spectrophotometer (BioTek). Extracts were aliquoted and stored at $-80{ }^{\circ} \mathrm{C}$.

\subsubsection{Measurement of protein concentration}

After the principle according to Bradford (1976), the concentration of proteins was measured. Therefore, Roti ${ }^{\circledR}$-Nanoquant (Roth) was employed as described by Witt 
(2013). This solution contains Coomassie Brilliant Blue that binds under acidic conditions to proteins which leads to a conversion of the red form to the blue form of the dye causing a shift in the absorption spectrum maximum from $365 \mathrm{~nm}$ to $595 \mathrm{~nm}$ (Bradford, 1976). A standard protein, namely BSA (0-6.7 $\mu \mathrm{g} \mathrm{BSA} / \mathrm{ml})$, was used for calibration. Based on the standard curve the protein concentration could be extrapolated. A triple measurement of each sample was performed and the average of the values was determined. First, a 1:100 dilution of the desired protein extract was prepared. $50 \mu \mathrm{l}$ of the diluted protein extract and $50 \mu \mathrm{l}$ of $\mathrm{ddH}_{2} \mathrm{O}$ as a reference were put in a 96 well plate and $200 \mu$ l of the Roti ${ }^{\circledR}$-Nanoquant solution was added. After 5 minute incubation at room temperature, the absorption was measured at 590 and $450 \mathrm{~nm}$ by Synergy Mx spectrophotometer.

\subsubsection{Co-IP}

The Co-IP is a technique used to analyse protein-protein interactions. With an antibody a known protein is targeted which is believed to be a member of a protein complex, the protein antigen is precipitated as well as proteins that are bound to it. By western blot analysis and further protein detection methods it might be possible to identify unknown complex members. For Co-IP nuclear cell extract was used and the immunoprecipitation of the target protein was carried out using the Immunoprecipitation Kit (Protein $G$ ) from Roche according to the manufacturer's instructions with modifications regarding incubation times, number of washing steps and during centrifugation steps. Depending on the amount of nuclear cell extract, an appropriate amount of the specific antibody was added and the sample incubated on a rotating platform overnight at $4{ }^{\circ} \mathrm{C}$ (Roche protocol: 1 hour). The next day $45 \mu 1$ of the protein $\mathrm{G}$ was added to a new tube and centrifuged for 1 minute at 13,000 rpm at room temperature (Roche protocol: $50 \mu \mathrm{l}$ ). The supernatant was removed and the overnight protein solution was added to the remaining protein G. Next, the mixture was incubated on a rotating platform for 2 hours at $4{ }^{\circ} \mathrm{C}$ (Roche protocol: 3 hours). Complexes were collected by 3 minutes centrifugation at 3,000 rpm at $4{ }^{\circ} \mathrm{C}$ (Roche protocol: $12,000 \mathrm{x} g$ for $20 \mathrm{sec}$ ). After the supernatant was removed, the beads were washed using $500 \mu \mathrm{l}$ of the lysis buffer (Roche protocol: 2 times $1 \mathrm{ml}$ lysis buffer and 20 minutes incubation). The samples were centrifuged again for 3 minutes at 3,000 rpm at $4{ }^{\circ} \mathrm{C}$ (Roche protocol: 12,000 x $g$ for $20 \mathrm{sec}$ ). The beads were resuspended in $1 \mathrm{ml}$ wash buffer 2 and centrifuged under the same conditions as 
before (Roche protocol: centrifugation at 12,000 x $g$ for $20 \mathrm{sec}$ after 20 minutes incubation). After the supernatant was removed, a final washing step using $1 \mathrm{ml}$ wash buffer 3 was performed (Roche protocol: 2 times $1 \mathrm{ml}$ wash buffer 3 and 20 minutes incubation). The following preparation to load the sample on an SDS gel was performed differently. The supernatant was removed after centrifugation and $18 \mu 1$ DPBS (PAN), $18 \mu 1$ LDS (Invitrogen) and $4 \mu 1$ 1M DTT were added to the beads. To denaturise proteins and separate them from the protein $G$ beads, the mixture was incubated for 5 minutes at $95{ }^{\circ} \mathrm{C}$. Beads were centrifuged down and the supernatant was loaded on an SDS gel and further analysed by western blot (2.2.17.5).

\subsubsection{SDS polyacrylamide gel electrophoresis}

By SDS polyacrylamide gel electrophoresis (SDS PAGE) proteins are denaturised and can be separated according to their molecular weight in a polyacrylamide gel by an electric field (Shapiro et al., 1967; Laemmli, 1970). In this work 4-12 \% Bis-Tris Gels (Invitrogen) and 3-8 \% Tris-Acetate Gels 3-8 \% Tris-Acetate Gels (Invitrogen) were used for protein separation (2.2.17.5).

\subsubsection{Western blot}

The western blot is an analytical technique and this method was used to transfer proteins from an SDS gel to a nitrocellulose membrane where the proteins can be detected by different reactions (Towbin et al., 1979; Towbin and Gordon, 1984; Towbin et al., 1992).

Protein extracts used for Co-IP were incubated with $18 \mu$ l DPBS (PAN), $18 \mu 1$ LDS (Invitrogen) and $4 \mu \mathrm{l} 1 \mathrm{M}$ DTT for 5 minutes at $95{ }^{\circ} \mathrm{C}$. After spinning down the Protein-G-agarose beads, $30 \mu \mathrm{l}$ of the supernatant which contains the precipitated protein along with other proteins bound to it in a denaturised form was loaded on an SDS gel. Two different transfer methods were used in this work.

\section{a) semidry}

With this transfer variant proteins $<100 \mathrm{kDa}$ were transferred from an SDS gel to a membrane. Using MES SDS Running Buffer (Invitrogen) and a 4-12 \% Bis-Tris Gel (Invitrogen), proteins were separated according to their molecular weight. For approximately $2.5-3$ hours the gel was running at $100 \mathrm{~V}$. The protein transfer was 
performed according to Wieczerzak (2012). The blotting condition was changed to $220 \mathrm{~mA}$ instead of $230 \mathrm{~mA}$. 4 Whatman Papers which were soaked in transfer buffer were placed on the Turboblotter ${ }^{\mathrm{TM}}$ (Schleicher \& Schuell). A nitrocellulose membrane which was wetted in $\mathrm{ddH}_{2} \mathrm{O}$ and transfer buffer was put on top of it followed by the SDS gel and another 4 prewetted Whatman Papers. Approximately $50 \mathrm{ml}$ transfer buffer were needed. The blotting was performed at constant $150 \mathrm{~mA}$ for 15 minutes followed by 45 minutes at $220 \mathrm{~mA}$.

\section{b) wet blot}

This transfer method is appropriate for larger proteins (> $100 \mathrm{kDa}$ ). First proteins were separated according to their molecular weight using gel electrophoresis. Therefore, Acetate SDS Running Buffer (Invitrogen) and 3-8 \% Tris-Acetate Gels (Invitrogen) were used. The running time of the gel amounted 3-3.5 hours at $100 \mathrm{~V}$. To prevent heating of the buffer, the gel chamber was placed in an ice box. Once the proteins were separated, the "gel-sandwich" was assembled in transfer buffer to avoid bubbles according to BIO-RAD (n.d.). On the grey coloured side of the cassette (Bio-Rad) a foam pad was placed followed by 2 Whatman Papers, a nitrocellulose membrane and the SDS gel. Finally, 2 Whatman Papers and another foam pad were placed on top. The cassette was closed and placed vertically between parallel electrodes in the buffer tank (Bio-Rad) containing a frozen blue cooling unit and the transfer buffer (Tab. 13). To avoid the formation of an ion gradient and to insure the temperature homogeneity, a magnetic stir bar was placed in the buffer tank which was put in a box filled with ice on a magnetic stirrer. For the blotting at $4{ }^{\circ} \mathrm{C}$, the voltage was set on $60 \mathrm{~V}$ constant for 2.5 hours.

\subsubsection{Protein detection using antibodies}

Once the protein transfer from the SDS gel to the membrane was completed, the membrane was transferred into a falcon tube (CellSTAR) and unspecific binding sites were blocked by incubating the membrane in TBST with $5 \%$ milk or $5 \%$ BSA for 1-2 hours at room temperature. During incubation steps membranes were placed on a rolling platform. The membrane was then incubated overnight at $4{ }^{\circ} \mathrm{C}$ with the appropriate primary antibody. Antibodies were diluted 1:1,000 to 1:2,000 in TBST with $2 \%$ milk or $2 \%$ BSA. Next day the membrane was washed three times for 10 minutes in $20 \mathrm{ml}$ TBST with $2 \%$ milk or TBST if BSA was used. The membrane 
was then incubated for 2 hours at room temperature or at $4{ }^{\circ} \mathrm{C}$ with a secondary antibody conjugated to horseradish peroxidase (HRP). The antibodies were 1:7,000 to $1: 10,000$ diluted in TBST with $2 \%$ milk (incubation at room temperature) or $2 \%$ BSA (incubation at $4{ }^{\circ} \mathrm{C}$ ). After that, the membrane was washed three times for 10 minutes in TBST with $2 \%$ milk and once with TBST for 5 minutes or three times for 10 minutes with TBST at room temperature. To visualise proteins, SuperSignal ${ }^{\mathrm{TM}}$ West Pico Chemiluminescent Substrate (Thermo Scientific) was used according to the company's instructions. To prepare the substrate working solution, the two substrate components were mixed in a 1:1 ratio (light sensitive). The membrane was placed between a clear plastic wrap and incubated 5 minutes in the dark with SuperSignal West Pico Substrate Working Solution. HRP produces a detectable light signal in the presence of a substrate which can be either detect on X-ray films or by the detecting system FlourChem ${ }^{\circledR} \mathrm{Q}$ (Alpha Innotech).

\subsubsection{Direct yeast two-hybrid}

The direct $\mathrm{Y} 2 \mathrm{H}$ assay is used to identify and investigate protein-protein interactions in vivo using yeast cells. Two proteins of interest are expressed separately. The protein functioning as a bait protein is fused to the Gal4 DNA-binding domain (e.g. pGBKT7 plasmid) while the other one functions as the prey protein which is fused to the Gal4 transcriptional activation domain (e.g. pGADT7 plasmid) (Fields and Song, 1989). Only if a cell contains both proteins that interact with each other, the Gal4 DNA activator is able to bind to the GAL4 DNA binding domain and activates the Gal4-responsive reporters ADE2 and HIS3 (Clontech_Laboratories, 2009a). That means the amino acids adenine and histidine are expressed. Furthermore, the bait plasmid encodes tryptophan (TRP1) (Clontech_Laboratories, 2008) and the prey plasmid encodes leucine (LEU2) (Clontech_Laboratories, 2012). By plating yeast cells on an appropriate synthetic dropout (SD) medium, transformants containing the introduced plasmid(s) can be selected. -LT SD-plates contain every essential amino acid except for leucine and tryptophan. They are used as a transformation control because only cells containing bait and prey plasmids are able to grow. To confirm a direct interaction of two proteins, co-transformed yeast cells are plated on -LTHA SD-plates (the medium includes every essential amino acid except for leucine, tryptophan, histidine and adenine). Only cells are able to grow that harbor bait and 
prey plasmids and additionally express proteins that interact with each other to activate HIS3 and ADE2, so the lacking nutritional factors in the medium can be expressed.

In this work the LiAc method (Ito et al., 1983) modified by Schiestl and Gietz (1989); Hill et al. (1991); Gietz et al. (1992) was used as a simple and highly reproducible method according to the Yeast Protocols Handbook (Clontech_Laboratories, 2009b) with slightly modifications.

Yeast cells (Y2H Gold, Clontech) from a working stock plate were inoculated in $25 \mathrm{ml}$ instead of $50 \mathrm{ml}$ yeast medium. The yeast containing medium was vigorously mixed to disperse the cells and cultured overnight (16-18 hours) at $30{ }^{\circ} \mathrm{C}$ shaking at $220 \mathrm{rpm}$ instead of $250 \mathrm{rpm}$. To get a mid-log phase culture, $4.5 \mathrm{ml}$ of the overnight culture $\left(\mathrm{OD}_{600}>1.5\right)$ was transferred to $150 \mathrm{ml}$ fresh yeast medium to have an $\mathrm{OD}_{600} \sim 0.2-0.3$ and cultured at $30{ }^{\circ} \mathrm{C}$ shaking at $220 \mathrm{rpm}$ (Clontech protocol: $230 \mathrm{rpm}$ ) for 3-5 hours until the culture had an $\mathrm{OD}_{600} \sim 0.5$. The culture was poured into $50 \mathrm{ml}$ tubes and centrifuged at 1,000 x $g$ for 5 minutes at room temperature. The supernatants were discarded and the pellets thoroughly resuspended in $\mathrm{ddH}_{2} \mathrm{O}$ and then pooled in one tube with a final volume of $25 \mathrm{ml}$. The sample was centrifuged again at $1,000 \times \mathrm{g}$ for 5 minutes at room temperature and the supernatant discarded. The cell pellet was resuspended in $1 \mathrm{ml}$ freshly prepared, sterile $1 \mathrm{x}$ TE/1 x LiAc (Clontech protocol: $1.5 \mathrm{ml}$ ). $1 \mu \mathrm{g}$ of each plasmid and $0.1 \mathrm{mg}$ of denaturised carrier DNA were mixed with $100 \mu 1$ of yeast competent cells. $600 \mu 1$ freshly prepared $40 \%$ PEG/1 x TE/1 x LiAc was added and vortexed. Probes were incubated at $30^{\circ} \mathrm{C}$ for 30 minutes shaking at $200 \mathrm{rpm}$. $70 \mu \mathrm{l}$ DMSO were added and mixed by gentle inversion. Next, a heat shock was performed at $42{ }^{\circ} \mathrm{C}$ for 15 minutes to promote the intake of plasmids into cells. After that, samples were incubated on ice for 1-2 minutes. Cells were centrifuged for 5 seconds at $14,000 \mathrm{rpm}$ at room temperature, the supernatant was discarded and the cells resuspended in $300 \mu 1$ $\mathrm{ddH}_{2} \mathrm{O}$ (Clontech protocol: $500 \mu \mathrm{l}$ TE buffer). Cells were plated on -LT SD agar plates as transformation control and on a -LTHA SD agar plate. Plates were incubated up-side-down at $30{ }^{\circ} \mathrm{C}$ for 3-7 days. 


\subsubsection{Cytological and histological methods}

\subsubsection{Immunocytochemistry with eukaryotic cells}

Immunocytochemistry (ICC) is a common method to detect proteins in cells using primary antibodies against the protein of interest. With a secondary antibody conjugated with a fluorophore, the localisation of the target protein can be visualised by a fluorescence microscope. The ICC was performed according to the ICC protocol from abcam with some modifications according to incubation times and concentrations (Abcam, n.d.).

One day before, HeLa cells were cultured on slides (BD Biosciences). The medium was removed and cells were washed twice with PBS. After that, cells were fixated for 20 minutes with $4 \%$ formaldehyde in PBS (abcam protocol: 15 minutes). If not indicated differently, all steps were carried out at room temperature. After the fixation cells were washed twice for 5 minutes with PBS and then permeabilised for 10 minutes using $0.1 \%$ Triton X-100 in PBS (abcam protocol: $0.25 \%$ Triton X100). After washing the cells twice with PBS for 5 minutes (abcam protocol: 3 times for 5 minutes), $3 \%$ BSA in PBS were added to the cells to block unspecific binding sides (abcam protocol: $1 \%$ BSA in PBST). After 20-30 minutes the blocking solution was removed and the primary antibody 1:100 diluted in 3\% BSA/PBS was added to the cells and incubated overnight at $4{ }^{\circ} \mathrm{C}$ (abcam protocol: $1 \%$ BSA in PBST). The next day the primary antibody dilution was removed and cells were washed twice for 10 minutes with PBS (abcam protocol: 3 times 5 minutes). The secondary antibody conjugated with Cy3 was diluted 1:200 in $3 \%$ BSA/PBS and added to the cells which were incubated afterwards for 2 hours (abcam protocol: $1 \%$ BSA for 1 hour). The last steps of the abcam protocol were performed differently. After washing the cells three times for 10 minutes in PBS and once with $d_{d d} \mathrm{H}_{2} \mathrm{O}$, Vectashield containing DAPI were added to the cells to stain the nuclei. The cells on the slide were closed by a cover slip and edges were sealed with nail polish.

\subsubsection{Duolink PLA}

The Duolink PLA method can be used to detect, visualise and quantify protein interaction in cells and tissues prepared for microscopy. The principle of this assay is based on in situ PLA $^{\circledR}$, a proximity ligation assay technology (OlinkBiosciences, 
2010). Two primary antibodies are used which are raised in two different species to detect the protein of interest. As secondary antibodies serve PLA probe PLUS and PLA probe MINUS which are conjugated with oligonucleotides (OlinkBiosciences, 2010). The two PLA probes generate a signal only when they bind to primary antibodies which have bound the target in close proximity (OlinkBiosciences, 2010). The signal is visible as an individual fluorescent spot.

In this work adherent human HeLa cells were used cultured in 8-well chamber slides (BD Biosciences) one day before starting the Duolink PLA method according to the manufacturer's instructions with some modifications. All steps were carried out at room temperature unless otherwise described. Cells were carefully washed twice with PBS to fully remove the medium. Next, the cells were fixed for 20 minutes in PBS with $3.7 \%$ formaldehyde. After cells were washed twice for 5 minutes in PBS, a permeabilisation step was performed for 10 minutes using PBS with $0.1 \%$ Triton X-100 to ensure access of the antibodies. Again, cells were washed twice for 5 minutes in PBS. To ensure that all non-specific binding sites are neutralised, cells were blocked in a preheated humidity chamber for 30 minutes at $37{ }^{\circ} \mathrm{C}$ with Blocking Solution (1 drop per reaction area), a component of the Duolink reagents. Primary antibodies were diluted 1:100 in Antibody Diluent which is another Duolink reagent. Cells were incubated overnight in a humidity chamber at $4{ }^{\circ} \mathrm{C}$ with diluted primary antibody combinations ( $30 \mu \mathrm{l}$ per reaction area): The next day the primary antibodies were tapped off the slides which were then washed three times instead of two times for 5 minutes with gentle shaking in cuvettes containing at least $70 \mathrm{ml}$ of Buffer A (prepared according to the manufacturer's instructions, Tab. 10). The two PLA probes were diluted 1:5 in Antibody Diluent and added to the cells (25 $\mu 1$ per reaction area). The slides were incubated in a preheated humidity chamber for 1 hour at $37{ }^{\circ} \mathrm{C}$. After the incubation, the PLA probe solution was tapped off the slides. Before adding the ligation-ligase solution to the cells, slides were washed three times instead of two times for 5 minutes with Buffer A. The $5 \mathrm{x}$ ligation stock was diluted 1:5 in $\mathrm{ddH}_{2} \mathrm{O}$. It contains two oligonucleotides that hybridise to the conjugated oligonucleotides of the PLA probes as well as all components necessary for ligation, except for the ligase. The ligase $(1 \mathrm{U} / \mu \mathrm{l})$ was diluted 1:40. The ligation-ligase solution was added to the cells ( $25 \mu 1$ per reaction area) which were then incubated in a preheated humidity chamber for 30 minutes at $37{ }^{\circ} \mathrm{C}$. The hybridised oligonucleotides will join to a close circle if the two PLA probes are in close 
proximity, acting as a template for the following amplification step. The slides were washed twice for 2 minutes in Buffer A. Next, the amplification solution consisting of the 1:5 diluted amplification stock (light sensitive!) and the 1:80 diluted Polymerase (10 U/ $\mu \mathrm{l})$ were added ( $30 \mu \mathrm{l}$ per reaction area) and slides were incubated in a preheated humidity chamber for 100 minutes at $37^{\circ} \mathrm{C}$. During the amplification reaction the ligated circle of oligonucleotides acts as a template for rolling-circle amplification (OlinkBiosciences, 2010). The oligonucleotide of one PLA probe functions as a primer (OlinkBiosciences, 2010). Fluorescently labeled oligonucleotides hybridise to the amplified product which will be visible as a distinct fluorescent spot under the fluorescence microscope indicating a protein interaction (OlinkBiosciences, 2010). After that slides were washed twice for 10 minutes in minimum $70 \mathrm{ml}$ Buffer B (prepared according to the manufacturer's instructions, Tab. 10) followed by an additional wash step with 0.1 x Buffer $B$ for 1 minute. Divergent from the manufacturer's protocol the cytoskeleton of the cells were stained with FITC Phalloidin (Sigma-Aldrich) using a 1:700 dilution in Antibody Diluent (20 $\mu 1$ per reaction area) for 30 minutes. After two final wash steps with $0.1 \times$ Buffer $\mathrm{B}$ for 10 minutes, the slides were air dried in the dark before they were mounted with cover slips and Mounting Medium containing DAPI (Vector) to stain the nuclei. The edges were sealed using nail polish. Slides were incubated at least 15 minutes in the dark before documented using a fluorescence microscope (Olympus) and a confocal laser scanning microscope (Olympus).

\subsubsection{Immunohistochemistry (3,3'-Diaminobenzidine (DAB) staining)}

For immunohistochemistry the Novocastra ${ }^{\mathrm{TM}}$ Novostain Universal Detection Kit (Leica) and DAB (Roche) were used after Batsukh et al. (2012) with slightly modifications. Paraffin sections on slides were deparaffinised by incubating 10 minutes in xylene followed by two additional incubating steps in fresh xylene for 5 minutes, respectively. Next, tissue samples were hydrated by a decreasing ethanol row $\left(100 \%, 95 \%, 90 \%, 80 \%, 70 \%\right.$ and $50 \%$ ethanol/ $\left.\mathrm{H}_{2} \mathrm{O}\right)$. Additionally to the procedure conducted by Batsukh et al. (2012), slides were rinsed carefully with $\mathrm{ddH}_{2} \mathrm{O}$ and then incubated for 5 minutes instead of 2 minutes in PBS. Next, slides were placed in a cuvette with an antigen retrieval buffer (Tab. 9) and boiled for 10 minutes in a steam cooker. Then, the cuvette with the slides was placed on ice for approximately 10-20 minutes. To block the endogenous peroxidase activity, the 
tissue samples were incubated with $3 \% \mathrm{H}_{2} \mathrm{O}_{2}$ in methanol instead of $6 \% \mathrm{H}_{2} \mathrm{O}_{2}$ in $\mathrm{H}_{2} \mathrm{O}$ for 15 minutes in a humidity chamber. After that, non-specific binding sites were blocked using prediluted horse serum (Leica). After 10 minutes incubation, the primary antibodies (1:50 to 1:100 diluted in 1:1 horse serum and TPBS (PBS + 0.2 \% Tween 20) were added on the tissue samples. Samples were incubated overnight (maximum 16 hours) at $4{ }^{\circ} \mathrm{C}$ in a humidity chamber. The next day the slides were taken from $4{ }^{\circ} \mathrm{C}$ and incubated for 1 hour at room temperature before they were washed for 5 minutes in PBS with $0.1 \%$ Tween 20. The prediluted biotinylated universal secondary antibody (Leica) was added to the tissue samples which were incubated for 10 minutes. After washing the slides for 5 minutes in PBS with $0.01 \%$ Triton X-100, tissue samples were incubated for 5 minutes in a prediluted Streptavidin peroxidase complex reagent (Leica). Streptavidin has a high affinity to biotin, so it binds to the biotinylated universal secondary antibody. Slides were washed again for 5 minutes in PBS with $0.1 \%$ Tween 20 and then tissue samples were incubated up to 2 minutes in $1 \times \mathrm{DAB}$ solution (Roche). DAB is oxidised by the peroxidase conjugated to Streptavidin which causes a brown colour. After washing the slides for 5 minutes in PBS with $0.1 \%$ Tween 20 instead of water, the nuclei were stained with haematoxylin. The slides were washed three times with $\operatorname{ddH}_{2} \mathrm{O}$, then covered with Aqua-Poly/Mount Coverslipping Medium (Polysciences) and a cover slip and the samples were analysed using a BX60 microscope (Olympus).

\subsubsection{Mutational analysis}

To screen for mutations in genes, the sequence of all coding exons and flanking intronic areas of the concerning gene was analysed by touchdown PCR (2.2.7.1). The PCR products were cleaned up by vacuum purification using MultiScreen Filter Plates (Merck Millipore). Each well of the plate was equilibrated with $100 \mu 1 \mathrm{TE}$ buffer. Another $100 \mu \mathrm{l} \mathrm{TE}$ buffer was added to the $25 \mu 1$ PCR reactions and mixed by pipetting. Then, each mixture was put onto a well of the plate. The vacuum pump (Merck Millipore) was turned on and the liquid in the wells were sucked off. To elute

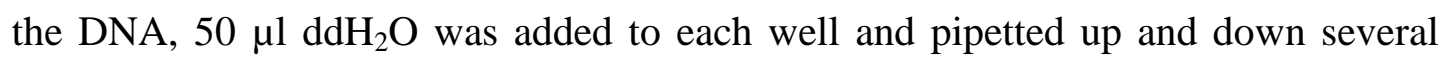
times. Next, the purified DNA was sequenced in both directions (2.2.7.2). Oligonucleotides used for touchdown PCR and sequencing are listed in table 20.

To screen for large deletions or duplications within a gene, qPCRs were performed for each coding exon (Tab. 33). 
2.2.21 Molecular biological methods concerning the model organism Xenopus laevis

\subsubsection{In vitro transcription of sense RNA}

To synthesise large amount of capped RNA that mimics most eukaryotic mRNAs in vivo, the mMESSAGE mMACHINE ${ }^{\circledR}$ SP6 or T7 Transcription Kits (Ambion) were used. These synthesised capped RNAs are ideal for oocyte microinjection (Ambion, 2012). The transcription reaction was assembled at room temperature according to the company's instructions. A $20 \mu 1$ transcription mixture was prepared using $1 \mu \mathrm{g}$ linearised plasmid template, $10 \mu 12 \times$ NTP/CAP, $2 \mu 110 x$ reaction buffer and $2 \mu 1$ $10 \mathrm{x}$ enzyme mix containing the appropriate RNA polymerase. The mixture was brought to a final volume of $20 \mu \mathrm{l}$ with nuclease free $\mathrm{H}_{2} \mathrm{O}$ and then mixed thoroughly and incubated 2 hours at $37{ }^{\circ} \mathrm{C}$. Next, the template DNA was removed by adding $1 \mu \mathrm{l}$ TURBO DNase $(2 \mathrm{U} / \mu \mathrm{l})$ during a 15 minutes incubation at $37{ }^{\circ} \mathrm{C}$. After the DNase treatment, the sense RNA was purified.

\subsubsection{Purification of sense RNA}

To purify sense RNA for oocyte microinjection, the Illustra ${ }^{\mathrm{TM}}$ RNAspin Mini (GE Healthcare) was used according to the manufacturer's instructions. All centrifugation steps were carried out at room temperature. To purify a $20 \mu 1$ transcription reaction, 3.5 volume of Buffer RA1 $(70 \mu \mathrm{l})$ and 95-100 \% ethanol $(70 \mu \mathrm{l})$, respectively were added, mixed and supplied on a column (blue). Next, samples were centrifuged at $8,000 \times \mathrm{g}$ for 30 seconds and the flow through was discarded. $200 \mu 1$ Buffer RA2 was added onto the column which was centrifuged at $11,000 \times \mathrm{x}$ for 1 minute. The column was placed in a new collection tube and $600 \mu 1$ Buffer RA3 was added. After centrifugation at $11,000 \times g$ for 1 minute, the flow through was discarded and $250 \mu 1$ of Buffer RA3 was supplied onto the column. To dry the membrane, the column was centrifuged at $11,000 \times \mathrm{g}$ for 2 minutes. The column was placed into a new $1.5 \mathrm{ml}$ tube. 33-35 $\mu$ l preheated RNase free $\mathrm{H}_{2} \mathrm{O}$ was added directly to the center of the membrane. The column was incubated for 2 minutes at $80{ }^{\circ} \mathrm{C}$ and then centrifuged at $11,000 \times \mathrm{g}$ for 1 minute. The concentration of sense RNA was determined using the NanoDrop 2000c spectrometer (Thermo Scientific) and stored at $-80{ }^{\circ} \mathrm{C}$. 


\section{Materials and methods}

\subsubsection{In vitro transcription of labeled anti-sense RNA}

For whole mount in situ hybridisation (WMISH) experiments digoxigenin (DIG) labeled anti-sense RNA was synthesised using a DIG-RNA labeling mixture (Roche) according to Hedderich (2012) with additional use of Pyrophosphatase, different amount of polymerases and modification of DNase incubation time. For a total transcription reaction of $25 \mu \mathrm{l}$ following chemicals were assembled (Tab. 37).

Table 37: Reaction conditions (in vitro transcription of labeled anti-sense RNA)

\begin{tabular}{c|c}
\hline volume & chemicals \\
\hline $5 \mu \mathrm{l}$ & $5 \mathrm{x}$ transcription buffer (Fermentas) \\
\hline $0.5 \mu \mathrm{l}$ & Pyrophosphatase $(4 \mathrm{U} / \mathrm{ml})$ \\
\hline $4 \mu \mathrm{l}$ & DIG-mix (ATP, CTP, GTP, UTP/digoxigenin UTP (Roche) \\
\hline $1 \mu \mathrm{l}$ & RNaseOut (40 U/ $\mu 1$, Invitrogen) \\
\hline $1 \mu \mathrm{l}$ & linearised template DNA \\
\hline $0.2-1 \mu \mathrm{g}$ & T7 or SP6 RNA polymerase $(20 \mathrm{U} / \mu \mathrm{l}$, Fermentas) \\
\hline $1 \mu \mathrm{l}$ & RNase free $\mathrm{H}_{2} \mathrm{O}$
\end{tabular}

The transcription reaction was incubated at $37{ }^{\circ} \mathrm{C}$ for 2 hours. To remove the template DNA, $1 \mu \mathrm{l}$ TURBO DNase $(2 \mathrm{U} / \mu \mathrm{l})$ was added and the sample was incubated at $37^{\circ} \mathrm{C}$ for 15 minutes. Next, the anti-sense RNA was purified.

\subsubsection{Purification of labeled anti-sense RNA}

To clean up labeled anti-sense RNA for oocyte microinjection, the RNeasy ${ }^{\mathrm{TM}}$ Mini Kit (Qiagen) was used according to manufacturer's manual. All centrifugation steps were carried out at room temperature. First, the transcription mixture was adjusted to a volume of $100 \mu \mathrm{l}$ using RNase free $\mathrm{H}_{2} \mathrm{O}$. Next, $350 \mu \mathrm{l}$ Buffer RLT was added and mixed well. After that, $250 \mu$ l ethanol (96-100 \%) was added to the diluted RNA and mixed well. The solution was transferred immediately onto an RNeasy Mini spin column that was centrifuged at $10,000 \mathrm{rpm}$ for 15 seconds. The flow-through was discarded and $500 \mu 1$ Buffer RPE was added to the column for washing. The column 
was centrifuged at 10,000 rpm for 15 seconds and the flow-throw was discarded. Again, $500 \mu \mathrm{l}$ Buffer RPE was added to the column that was centrifuged at 10,000 rpm for 2 minutes. The column was placed into a new $2 \mathrm{ml}$ collection tube and centrifuged at full-speed for 1 minute. The column was placed into a new $1.5 \mathrm{ml}$ receiver tube. $50 \mu \mathrm{l}$ preheated $\mathrm{RNase}$ free $\mathrm{H}_{2} \mathrm{O}$ was added to the center of the membrane and the column was incubated at $80^{\circ} \mathrm{C}$ for 2 minutes. To elute the RNA, the column was centrifuged at $10,000 \mathrm{rpm}$ for 1 minute. To determine the concentration of the anti-sense RNA, the NanoDrop 2000c spectrometer (Thermo Scientific) was used. The anti-sense RNA was stored at $-80^{\circ} \mathrm{C}$.

\subsubsection{Morpholino oligonucleotides}

The Morpholino oligonucleotides (MO) were purchased from Gene Tools, LLC. The MO were dissolved at $65{ }^{\circ} \mathrm{C}$ in $\mathrm{RNase}$ free $\mathrm{H}_{2} \mathrm{O}$ and diluted to a final concentration of $8 \mu \mathrm{g} / 10 \mathrm{nl}$. MO were stored at $4{ }^{\circ} \mathrm{C}$ and heated up for 10 minutes at $65{ }^{\circ} \mathrm{C}$ before usage.

\subsubsection{Preparation of Xenopus laevis testis and fertilisation of oocytes}

The preparation of the testis and fertilisation of oocytes have been carried out by Dr. Peter Wehner according to Wehner (2012). To isolate sperm from testis, male Xenopus laevis were put into a $0.05 \%$ benzocaine/water solution for 30 minutes at room temperature. Frogs were decapitated and the testis was removed, then washed three times with MBS and stored in $1 \mathrm{x}$ MBS buffer at $4{ }^{\circ} \mathrm{C}$. To stimulate the egg deposition, female Xenopus laevis frogs were injected into the dorsal lymph sac with 1,000 units human chorionic gonadotropin (hCG) hormone (Sigma-Aldrich). Approximately 12 hours after injection the female frogs lay eggs. These eggs were fertilised in vitro. Therefore, a piece of testis was macerated in $0.1 \mathrm{x}$ MBS. To remove the jelly coat from fertilised eggs, they were incubated for 3-5 minutes with $2 \%$ cysteine hydrochloride ( $\mathrm{pH} 8)$.

\subsubsection{Microinjection of Xenopus laevis embryos and culture}

Microinjections of Xenopus laevis blastomeres have been performed by Dr. Peter Wehner according to Wehner (2012). For microinjection fertilised eggs were put into injection buffer and placed on a cooling plate. Glass capillaries that were prepared with a needle puller were loaded with the substances needed for microinjection. The 
injection was carried out using the Microinjector 5242 (Eppendorf). 4-10 nl $\beta$ galactosidase (lacZ) mRNA (W. C. Smith and Harland, 1991) and MO were injected animally into one blastomere of a two-cell stage embryo. The MO used are short oligonucleotides that interfere with the target mRNA to block translation. To allow the heeling after injection, embryos were kept for 1-2 hours in injection buffer. After that, they were washed twice with 0.1 x MBS. Embryos were cultured to the desired stage in $0.1 \mathrm{x}$ MBS at 12.5 to $18{ }^{\circ} \mathrm{C}$. The developmental stages were determined according to Nieuwkoop and Faber (Hubrecht-Laboratorium (Embryologisch Instituut) et al., 1967).

\subsubsection{Fixation of injected Xenopus laevis embryos and X-gal staining}

Fixation and X-gal staining of Xenopus laevis embryos have been performed by Dr. Peter Wehner and me according to Wehner (2012). This procedure is used for WMISH to determine the lacZ mRNA injected region of the embryo (Hardcastle et al., 2000). The lac $Z$ mRNA is co-injected as a lineage tracer with the appropriate MO. After injected embryos developed to the desired stage, they were fixated for 1 hour in MEMFA. All steps were carried out at room temperature. After the fixation, embryos were washed three times for 10 minutes with 1 x PBS and then stained with X-gal solution in the dark until the requested intensity of staining was achieved. The X-gal solution was removed and embryos were washed three times for 10 minutes in 1 x PBS. Next, embryos were fixed again for 1 hour in MEMFA. Afterwards, embryos were washed three times with $100 \%$ ethanol. Embryos were long term stored in $100 \%$ ethanol at $-20{ }^{\circ} \mathrm{C}$.

\subsubsection{Whole mount in situ hybridisation}

With this technique the localisation of mRNA within an embryo can be visualised using a labeled specific complementary RNA probe. The WMISH was performed as described by Harland (1991) with some modifications.

Detailed procedure of the WMISH on Xenopus laevis:

\section{Day 1}

All incubation steps were performed under gentle shaking. Xenopus laevis embryos were rehydrated by a decreasing ethanol row $\left(75 \% \mathrm{EtOH} / \mathrm{H}_{2} \mathrm{O}, 50 \% \mathrm{EtOH} / \mathrm{H}_{2} \mathrm{O}\right.$, 
$25 \% \mathrm{EtOH} / \mathrm{PTw}$ ) followed by five washing steps with PTw. Each step was carried out for 5 minutes at room temperature.

To permeabilise the embryos to ensure fully penetration of RNA probes, embryos were treated with proteinase $\mathrm{K}(10 \mu \mathrm{g} / \mathrm{ml})$ in PTw as described in table 38 .

Table 38: Proteinase $\mathrm{K}$ treatment of Xenopus laevis embryos

\begin{tabular}{c|c|c}
\hline $\begin{array}{c}\text { Developmental stage of } \\
\text { Xenopus laevis embryos }\end{array}$ & Incubation time (min) & Temperature \\
\hline $9-10.5$ & $6-8$ & room temperature \\
\hline $14-16$ & $8-10$ & room temperature \\
\hline $20-25$ & $15-18$ & room temperature \\
\hline 36 & $22-25$ & room temperature \\
\hline 40 & $17-20$ & $37^{\circ} \mathrm{C}$ \\
\hline $42-43$ & $27-30$ & $37^{\circ} \mathrm{C}$ \\
\hline 46 & $32-35$ & $37^{\circ} \mathrm{C}$ \\
\hline
\end{tabular}

The following acetylation step increases the specific binding of the probe to mRNA. Therefore, the embryos were washed twice in $0.1 \mathrm{M}$ Triethanolamine $(\mathrm{pH} 7.5$ ) for 5 minutes, respectively. After that, embryos were incubated for 5 minutes in $4 \mathrm{ml}$ Triethanolamine with $12.5 \mu \mathrm{l}$ Acetic Anhydride. Additional $12.5 \mu \mathrm{l}$ Acetic Anhydride was added followed by two 5 minute washing steps with PTw.

Subsequent to the acetylation embryos were fixed for 20 minutes in PTw containing $4 \%$ formaldehyde and then washed five times with PTw buffer for 5 minutes, respectively.

For the following hybridisation embryos were left in $1 \mathrm{ml}$ PTw and $250 \mu \mathrm{l}$ prewarmed Hybmix was added and carefully mixed. The solution was replaced by $1 \mathrm{ml}$ fresh Hybmix and after 10 minutes incubation at $60{ }^{\circ} \mathrm{C}$ replaced again by $1 \mathrm{ml}$ Hybmix. After 4-5 hours incubation at $60{ }^{\circ} \mathrm{C}$, the Hybmix was exchanged and the embryos hybridised over night at $60{ }^{\circ} \mathrm{C}$ with the desired antisense RNA probe diluted in Hybmix. 


\section{Day 2}

The next day the RNA probe was removed and preheated Hybmix was added to the embryos which were incubated for 10 minutes at $60^{\circ} \mathrm{C}$. After that, the embryos were incubated three times for 15 minutes in $2 \times \mathrm{SSC}$ at $60^{\circ} \mathrm{C}$. An RNase mix containing $2 \times$ SSC, $10 \mu \mathrm{g} / \mathrm{ml}$ RNase A and $0.01 \mathrm{U} / \mathrm{ml}$ RNase T1 was added to the embryos to degrade mismatched double stranded RNA. After an incubation time of approximate 40 minutes at $37{ }^{\circ} \mathrm{C}$ and $30 \mathrm{rpm}$, the RNase mix was washed away with $2 \times$ SSC for 5 minutes at room temperature followed by two additional washing steps with $0.2 \mathrm{x}$ SSC for 30 minutes at $60{ }^{\circ} \mathrm{C}$ as well as two further wash steps with 1 x MAB for 15 minutes at room temperature.

Next, the antibody reaction took place. First, embryos were washed once with MAB containing $2 \%$ of the blocking reagent $\mathrm{BMB}$ for 15 minutes at room temperature followed by 40 minutes incubation in MAB containing $2 \% \mathrm{BMB}$ and $20 \%$ horse serum at room temperature. The next 4 hours the embryos were treated with MAB containing $2 \%$ BMB, $20 \%$ horse serum and 1:5,000 diluted Sheep Alkaline phosphatase-coupled anti-Dig antibody at room temperature. After the antibody incubation, the embryos were washed three times with MAB for 10 minutes at room temperature. Embryos were kept in fresh MAB over night at $4{ }^{\circ} \mathrm{C}$.

\section{Day 3}

The next day, the washing with MAB was continued five times for 5 minutes at room temperature, respectively. In preparation to the colour reaction the embryos were washed twice for 5 minutes at room temperature with fresh prepared APB. For the colour reaction itself the embryos were incubated up to 3 days at $4{ }^{\circ} \mathrm{C}$ in $3 \mathrm{ml} \mathrm{APB}$ solution containing $2.4 \mu \mathrm{l} \mathrm{NBT}(100 \mathrm{mg} / \mathrm{ml})$ and $10.5 \mu \mathrm{l} \mathrm{BCIP}(50 \mathrm{mg} / \mathrm{ml})$.

To reduce background after staining, a decreasing methanol row (one minute or longer in $100 \%$ methanol, one minute in $75 \%, 50 \%$ and $25 \%$ methanol/ $/ \mathrm{H}_{2} \mathrm{O}$, respectively) can be performed. After removing the background, the pigmented embryos were bleached at room temperature to remove the pigmentation which can interfere the staining. Therefore, the embryos were fixated in MEMFA for 30 minutes and washed twice for 5 minutes in $5 \mathrm{x}$ SSC. Next, the embryos were bleached up to 30 minutes in $5 \times$ SSC containing $50 \%$ formamide and $2 \% \mathrm{H}_{2} \mathrm{O}_{2}$. Finally, embryos were washed twice for 5 minutes in $5 \mathrm{x}$ SSC and 30 minutes in 
Materials and methods

MEMFA. Before documentation, the embryos were washed three times for 5 minutes in PTw. Embryos were stored in $100 \%$ ethanol at $-20{ }^{\circ} \mathrm{C}$. 


\subsubsection{OVERVIEW}

\section{Co-IP}

generation of constructs (recloning of the gene of interest in the pCMV-HA vector)

- PCR to amplify the plasmid with gene of interest

- gel extraction

- restriction digestion

- ethanol precipitation

- cloning by In-Fusion ${ }^{\mathrm{TM}}$ or ligation

- transformation

- mini-preparation of plasmid with appropriate insert

- test digestion

- purification of mini-preparation

- sequencing

- midi-preparation of plasmid with appropriate insert

transfection of HeLa cells

$>$ isolation of nuclear proteins from HeLa cells

$>$ Co-IP

separation according to the molecular weight on SDS gel

western blot

$>$ antibody treatment

$>$ detection of protein signals

\section{Immunocytochemistry}

culture HeLa cells on slides

$>$ fixation of cells

$>$ permeabilisation of cells

blocking

antibody treatment

$>$ data analysis

\section{Duolink}

culturing HeLa cells on 8-well chamber slides

fixation of cells 


\section{Materials and methods}

permeabilisation of cells

Duolink

staining the cytoskeleton of cells with FITC Palloidin and the nuclei with DAPI

data analysis (fluorescence microscope/confocal laser scanning microscope)

\section{Yeast two-hybrid}

generation of constructs

- PCR to amplify the gene of interest

- gel extraction

- restriction digestion of the plasmid

- if necessary ethanol precipitation and treatment with Antarctic phosphatase

- cloning by In-Fusion ${ }^{\mathrm{TM}}$

- transformation

- mini-preparation of plasmid with appropriate insert

- test digestion

- purification of mini-preparation

- sequencing

- (midi-preparation of plasmid with appropriate insert)

Yeast two-hybrid

\section{Transcriptome, micro array}

mating heterozygous $\left(C h d 7^{\text {Whi/+ }}\right)$ female and male mice

preparation of E9.5 embryos

$>$ tail biopsy for genotyping and shock freezing of the embryo

$>$ determination of the genotype

- isolating of genomic DNA

- genotyping PCR

- test agarose gel

- purification of genotyping PCR samples

- sequencing PCR

determination of gender by PCR

RNA isolation of E9.5 embryos

$>$ micro array 
data analysis

\section{Confirmation of micro array by RT-qPCR}

mating heterozygous $\left(C h d 7^{\text {Whil+}}\right)$ female and male mice

$>$ preparation of E9.5 embryos

$>$ tail biopsy for genotyping and shock freezing of the embryo

$>$ determination of the genotype

$>$ determination of gender by PCR

$>$ RNA isolation of E9.5 embryos

$>$ reverse transcription (cDNA synthesis)

$>$ test primer for RT-qPCR by PCR

$>$ RT-qPCR

$>$ data analysis

\section{DAB staining}

mating wild-type female and male mice of the CD-1 strain

$>$ preparation of E12.5 embryos

$>$ fixating embryos overnight in $4 \%$ paraformaldehyde/PBS

$>$ prepare embryos for embedding in paraffin

$>$ paraffin sections

preparing the sections for immunostaining with DAB

- Xylenel treatment and rehydration

- boiling in EDTA

- blocking step and antibody incubation

- DAB staining

$>$ data analysis

\section{Patient screen}

\section{Mutation screen}

generate primers for each exon of the gene of interest

$>$ PCR to amplify each exon of the gene of interest

$>$ purify PCR samples

$>$ sequencing

$>$ data analysis 


\section{Deletion/Duplication screen}

generating primers for each exon of the gene of interest

$>$ test primers in a qPCR using wild-type genomic DNA

$>$ measurement of DNA concentration of patient samples

qPCR

$>$ data analysis

\section{Whole mount in situ hybridisation}

generating sense and labeled anti-sense probes

- digestion of plasmid with the appropriate gene

- purification of digested plasmids

- in vitro transcription of sense and labeled anti-sense RNA

- purification of sense and labeled anti-sense RNA

- measurement of RNA concentration of sense and labeled anti-sense RNA

injection of one blastomere of a two-cell Xenopus laevis oocytes with MO

$>$ collection of embryos at the desired stage

$>$ fixation and X-gal staining of embryos

$>$ whole mount in situ hybridisation

$>$ data analysis 


\section{Results}

\subsection{CHD7 interacts with components of the WAR complex}

Chromatin remodelling enzymes are known to form large multi-subunit complexes (Mohrmann and Verrijzer, 2005; Ho et al., 2009a). It was shown that CHD7 interacts with the chromatin remodelling enzyme CHD8, which in turn interacts with components of the WAR complex (Batsukh et al., 2010; Yates et al., 2010). The following described data are published by us (Schulz et al., 2014a). To prove whether CHD7 also interacts with the members of the WAR complex WDR5, ASH2L and RBBP5, Co-IP experiments on human HeLa cells were performed (Fig. 5).

HeLa cells were single transfected with pCMV-HA plasmids containing the human full-length sequence of ASH2L (NP_004665.2) or RBBP5 (NP_005048.2). Untransfected HeLa cells were used as a negative control. Using a CHD7 antibody, endogenous CHD7 was precipitated. With an HA antibody the overexpressed proteins ASH2L (Fig. 5A, lane 1) and RBBP5 (Fig. 5A, lane 2) were detected. As expected no band was observed for the negative control (Fig. 5A, lane 3). Bands of the appropriate size were observed after detecting the overexpressed proteins ASH2L and RBBP5 with an HA antibody, confirming the successful transfection (Fig. 5A, lane 4+5). Co-IPs where endogenous CHD8 was precipitated with a CHD8 antibody and overexpressed ASH2L (Fig. 5B, lane 1), RBBP5 (Fig. 5B, lane 2) and the CHD7 fragment (amino acids 1591-2181, NP_060250.2) (Fig. 5B, lane 3) were detected with an HA antibody confirmed the known interaction of CHD8 and CHD7 as well as CHD8 and the WAR complex members. The reciprocal experiment was performed by single transfection of HeLa cells with pCMV-HA plasmids containing either ASH2L or RBBP5. Untransfected HeLa cells served as a negative control. Next, the overexpressed proteins were precipitated using an HA antibody. With a CHD7 antibody endogenous CHD7 was detected at $\sim 320 \mathrm{kDa}$. A band at the estimated size was observed after ASH2L (Fig. 5C, lane 1) and RBBP5 single transfection (Fig. 5C, lane 2). No band was observed for the negative control, showing that endogenous CHD7 does not bind unspecifically to the beads (Fig. 5C, lane 3). Untransfected HeLa cells were used to precipitate endogenous WDR5 using a WDR5 antibody. With a CHD7 antibody the estimated size of endogenous CHD7 
$(\sim 320 \mathrm{kDa}$ ) was detected (Fig. 5C, lane 4). Furthermore, endogenous CHD7 was detected in untransfected HeLa cells used as an input control (Fig. 5C, lane 5). In conclusion, the data obtained by Co-IP experiments demonstrate that CHD7 interacts with WDR5, ASH2L and RBBP5.

\section{A}

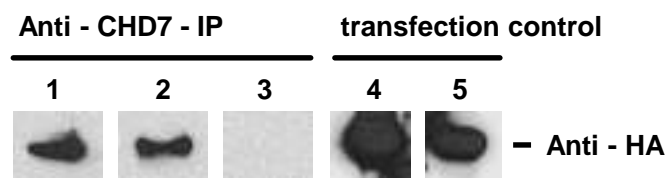

B

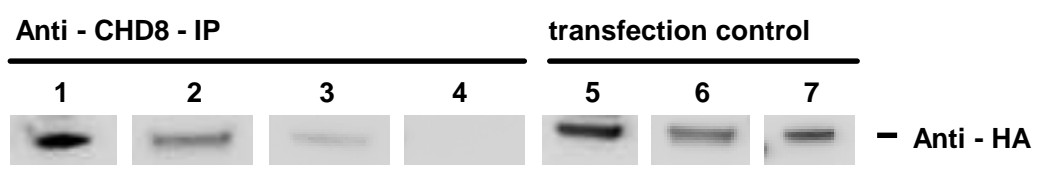

C

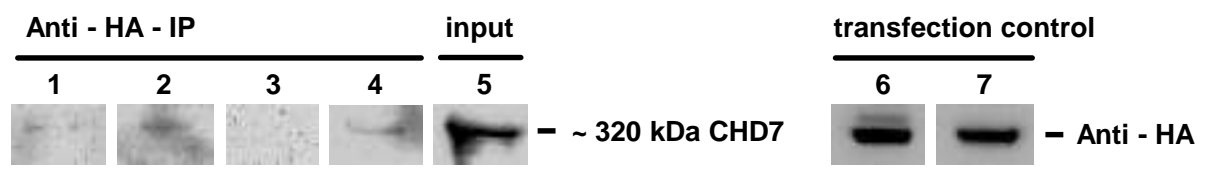

Figure 5: Co-immunoprecipitation experiments with HeLa cells.

(A) HeLa cells were single transfected with the pCMV-HA plasmids containing either the human fulllength sequence of ASH2L (NP_004665.2) (lane 1) or RBBP5 (NP_005048.2) (lane 2). Endogenous CHD7 was precipitated using a CHD7 antibody. With an HA antibody bands of the estimated size of ASH2L ( $76 \mathrm{kDa}$; lane 1) and RBBP5 ( $59 \mathrm{kDa}$; lane 2) were observed. Untransfected HeLa cells served as a negative control (lane 3). After precipitating endogenous CHD7, no band was detected with an HA antibody. Lane 4 and 5 represent the transfection controls: input after ASH2L-pCMV-HA single-transfection of HeLa cells (lane 4); input after RBBP5-pCMV-HA single transfection of HeLa cells (lane 5). Overexpressed ASH2L and RBBP5 were detected with an HA antibody. (B) Positive control, confirming the interaction of CHD8 with ASH2L, RBBP5 and CHD7. HeLa cells were single transfected with ASH2L-pCMV-HA (lane 1), RBBP5-pCMV-HA (lane 2) or CHD7-CR1-3-pCMVHA (NP_060250.2) (lane 3). The pCMV-HA plasmids contain the human full-length sequence of ASH2L or RBBP5 or a part of CHD7 (amino acids 1591-2181). A CHD8 antibody was used for precipitation of endogenous CHD8 and an HA antibody for detection of the overexpressed proteins. A band at $\sim 76 \mathrm{kDa}$ corresponding to the estimated size of ASH2L (lane 1), a band ( $59 \mathrm{kDa}$ ) of the predicted size of RBBP5 (lane 2) and a band ( $70 \mathrm{kDa}$ ) of the CHD7 part was detected. As a negative control untransfected HeLa cells were used (lane 4). Endogenous CHD8 was precipitated with a CHD8 antibody. No band was observed by using an HA antibody for detection (lane 4). Lane 5-7 show the transfection controls. Input after single transfection of HeLa cells with ASH2L-pCMV-HA (lane 5), RBBP5-pCMV-HA (lane 6) and CHD7-CR1-3-pCMV-HA (lane 7). The overexpressed 
proteins were detected with an HA antibody. (C) Vice versa experiment to experiment A. HeLa cells were single transfected with the plasmids ASH2L-pCMV-HA (lane 1) or RBBP5-pCMV-HA (lane 2). The overexpressed proteins were precipitated with an HA antibody and endogenous CHD7 ( $320 \mathrm{kDa})$ was detected using a CHD7 antibody. Untransfected HeLa cells were used as a negative control (lane 3). No CHD7 was detected after precipitation with an HA antibody, indicating that endogenous CHD7 does not bind unspecifically to the beads. Endogenous WDR5 was precipitated with a WDR5 antibody using untransfected HeLa cells and a $\sim 320 \mathrm{kDa}$ band was observed with a CHD7 antibody (lane 4). The transfection controls are shown in lane 6 and 7. Overexpressed ASH2L (lane 6) and RBBP5 (lane 7) were detected with an HA antibody after single transfection of HeLa cells with either ASH2L-pCMV-HA or RBBP5-pCMV-HA. Three biological replicates were performed for each Co-IP experiment (A - C). Figure modified after Schulz et al. (2014a).

\subsection{CHD7 and the members of the WAR complex are co-localised in the nucleus}

To confirm the Co-IP data and determine where in the cell interactions occur, the Duolink PLA method was used. The antibodies used to detect the target proteins were tested in an immunocytochemistry performed on untransfected HeLa cells. The results are shown in figure 6. The cell nuclei were stained with DAPI. Primary antibodies were used to detect CHD7, CHD8, WDR5, ASH2L and RBBP5. With secondary antibodies conjugated with $\mathrm{Cy} 3$ the localisation of the proteins was visualised (Fig. 6B, E, H, K, L). Merging the DAPI (nuclei) and the Cy3 channel (detected target proteins) revealed that all analysed proteins were localised specifically in the nucleus: CHD7 (Fig. 6C), CHD8 (Fig. 6F), WDR5 (Fig. 6I), ASH2L (Fig. 6L), RBBP5 (Fig. 6O). 


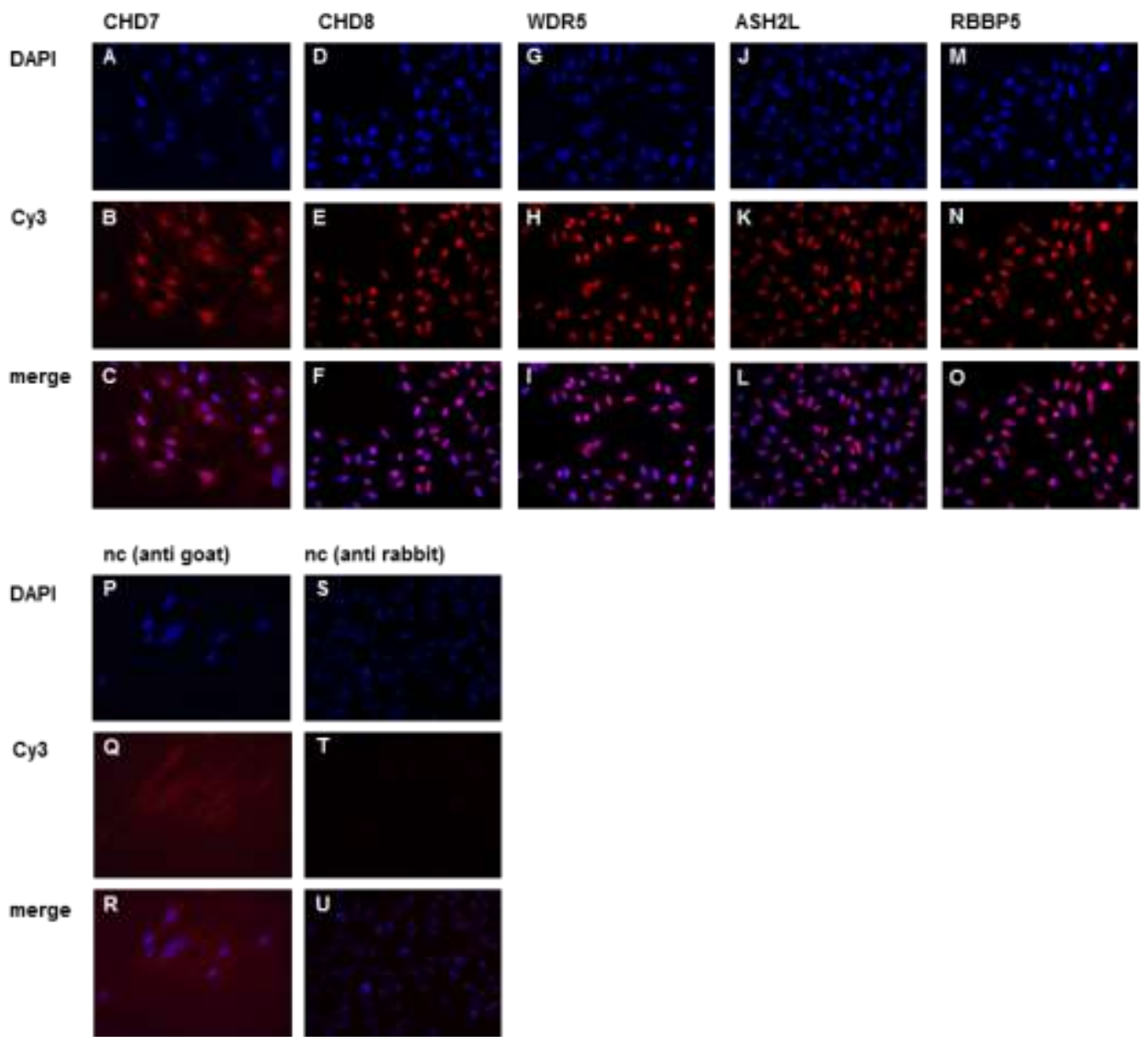

Figure 6: Protein localisation after immunocytochemistry performed with HeLa cells.

Untransfected HeLa cells were incubated overnight with following antibodies to detect the target proteins CHD7 (B, C), CHD8 (E, F), WDR5 (H, I), ASH2L (K, L) and RBBP5 (N, O). Cy3 conjugated secondary antibodies (anti-goat: B, C and anti-rabbit: E, F, H, I, K, L, N, O) were used for visualisation. All proteins analysed were detected in the nucleus. The nuclei of cells were stained with DAPI (A, D, G, J, M, P, S). To validate the specificity of the secondary antibody, HeLa cells were incubated only with the $\mathrm{Cy} 3$ conjugated antibody (anti-goat: P-R and anti-rabbit: S-U). It was shown that the anti-goat $\mathrm{Cy} 3$ antibody causes a slightly background $(\mathrm{Q}, \mathrm{R})$.

For analysing protein interactions by the Duolink PLA method, untransfected HeLa cells were used. The results are presented in figure 7 and published by us (Schulz et al., 2014a). Cell nuclei were stained with DAPI and the cytoskeleton of cells with FITC Phalloidin. PLA signals (red dots) indicate the protein interactions. By merging the channels the localisation of the protein interaction within the cells could be determined. Positive PLA signals were detected in the nucleus using antibodies against CHD7 and WDR5 (Fig. 7C, D, E), CHD7 and ASH2L (Fig. 7H, I, J), CHD7 and RBBP5 (Fig. 7M, N, O), CHD7 and CHD8 (Fig. 7R, S, T). The known interaction between CHD7 and CHD8 were used as a positive control. Cells only 
treated with one primary antibody served as a negative control. Only few PLA signals were observed in the cytoplasm and nuclei showing no specific preference, indicating that nearly no unspecific binding of the PLA probes to the antibodies is present (Fig. 7U, V, W, X, Y). In summary, the interaction of CHD7 with the members of the WAR complex WDR5, ASH2L and RBBP5 was confirmed by Duolink PLA. Furthermore, it was shown that the interactions were localised in the nuclei of HeLa cells.

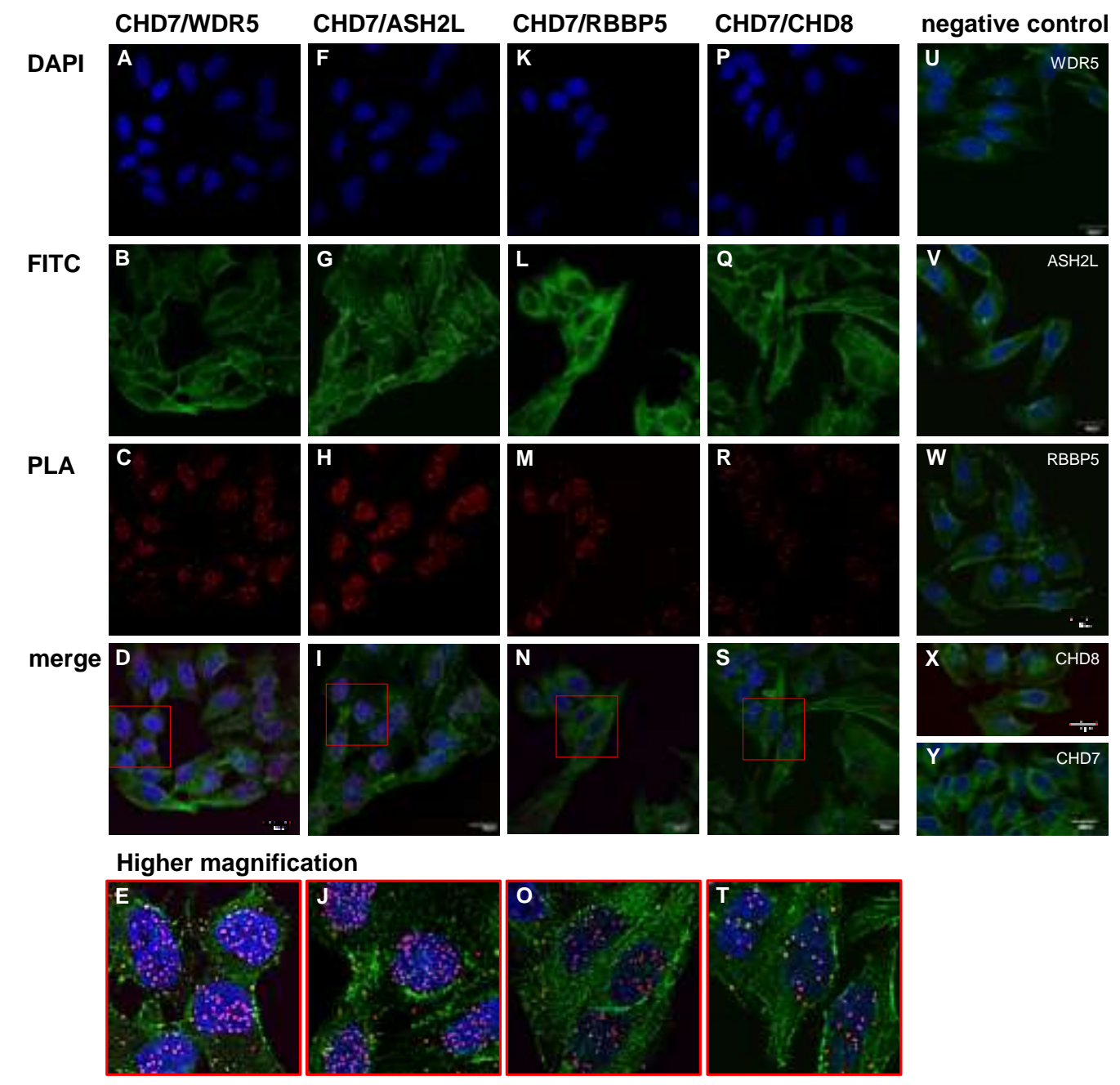

Figure 7: Analysing protein interactions in HeLa cells using the Duolink PLA method.

The nuclei of cells were stained with DAPI (A, F, K, P) and the cytoskeleton of cells were stained with FITC Phalloidin (B, G, L, Q). Positive PLA signals (red dots) indicate protein interactions. PLA signals were mainly detected in the nuclei using antibodies against CHD7 and WDR5 (C, D, E), CHD7 and ASH2L (H, I, J), CHD7 and RBBP5 (M, N, O) and CHD7 and CHD8 (R, S, T) which was used as a positive control. A higher magnification of the merged pictures is shown (E, J, O, T). Only few PLA signals were observed in the cytoplasm and nuclei with no specific preference using an antibody against WDR5 (U), ASH2L (V), RBBP5 (W), CHD8 (X) or CHD7 (Y). These results indicate that there is nearly no unspecific binding of the PLA probes to the antibodies. The Duolink 
PLA experiment was carried out three times. Scale bars indicated in the merged pictures correspond to $20 \mu \mathrm{m}$. Figure modified after Schulz et al. (2014a).

\subsection{CHD7 shows no direct interaction with WAR complex members}

To further analyse whether the identified interactions of CHD7 with components of the WAR complex are direct ones, Y2H experiments were carried out (Fig. 8) and published by us (Schulz et al., 2014a). The recently described constructs CHD7CR1-3-pGBKT7 containing the CHD7 fragment spanning the amino acids 15912181 (NP_060250.2) and the CHD8-pGBKT7 plasmid containing a CHD8 part (amino acids 1789-2302, NP_065971.2) were used as bait plasmids (Batsukh et al., 2010). WDR5-pGADT7 (NP_060058.1), ASH2L-pGADT7 (NP_004665.2) and RBBP5-pGADT7 (NP_005048.2) served as prey plasmids, respectively. Colonies on the -LT plates indicated a successful transformation (Fig. 8A, C, D, F). As a positive control the CHD8-pGADT7-Rec prey plasmid and the CHD7-CR1-3-pGBKT7 bait plasmid were used (Batsukh et al., 2010). The auto activation test using the empty pGBKT7 plasmid and pGADT7 with WDR5, ASH2L or RBBP5 revealed no auto activation of the yeast strain reporter genes (Fig. 8C, E, G). While the direct interaction of the recently described CHD8 fragment (Batsukh et al., 2010) and WDR5, ASH2L and RBBP5 was confirmed, the direct $\mathrm{Y} 2 \mathrm{H}$ experiments revealed no direct interaction of the CHD7-CR1-3-part with the WAR complex members (Fig. 8B, E, G). 
Results

A

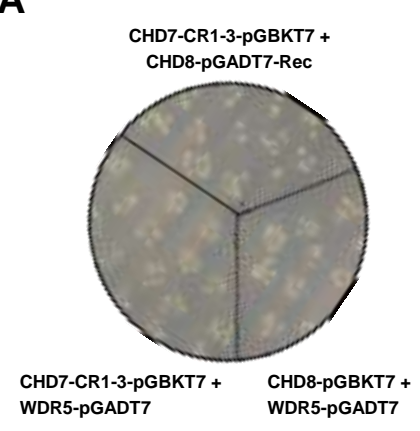

D

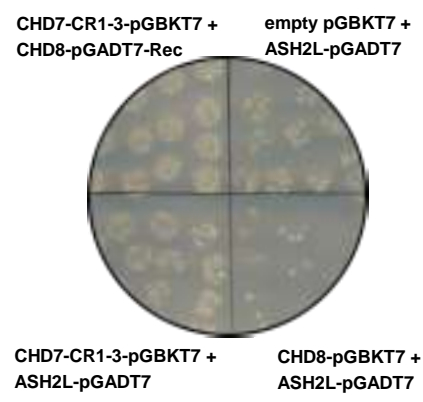

$\mathbf{F}$

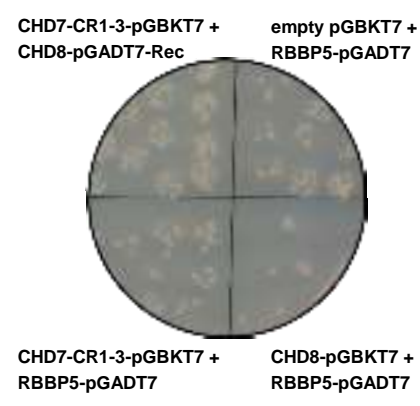

- LT
B

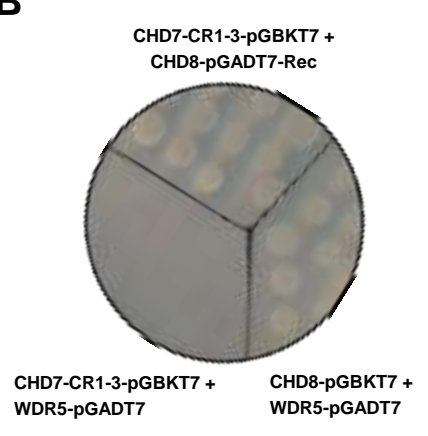

E

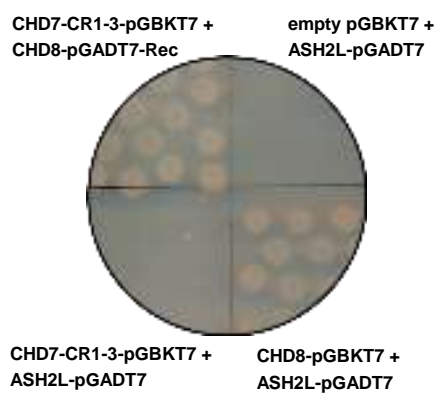

G

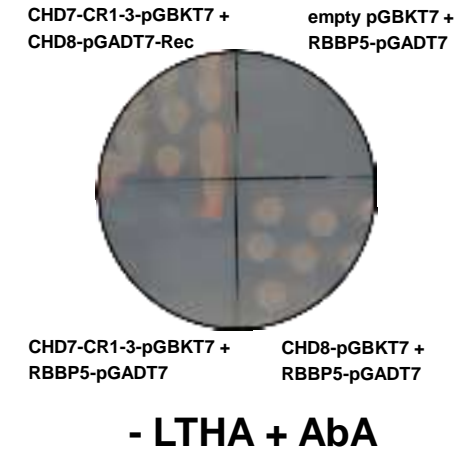

C

empty pGBKT7 + WDR5-pGADT7 (auto activation test)

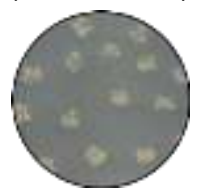

- LT

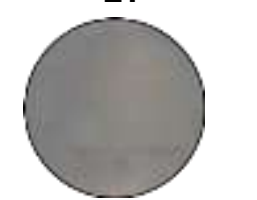

- LTHA + AbA

Figure 8: Results of the direct yeast two-hybrid assay.

CHD7-CR1-3-pGBKT7 was used as a bait plasmid to analyse a direct interaction of the CHD7 part (amino acids 1591-2181, NP_060250.2) and the components of the WAR complex (WDR5, ASH2L and RBBP5). The pGADT7 prey plasmid contained either the full-length sequence of WDR5 (NP_060058.1), ASH2L (NP_004665.2) or RBBP5 (NP_005048.2). CHD7-CR1-3-pGBKT7 and CHD8-pGADT7-Rec (containing CHD8 amino acids 1789-2302, NP_065971.2) were used as a positive control. Colonies for each sample on the -LT plate indicated a successful transformation (A, C, D, F). No colonies were detected on -LTHA + Aba (Aureobasidin A) plates using the empty pGBKT7 plasmid and the pGADT7 plasmid with WDR5, ASH2L or RBBP5, showing no auto activation of the yeast strain reporter genes (C, E, G). The direct interaction of the CHD8 part (CHD8pGBKT7, amino acids 1789-2302, NP_065971.2) and the WAR complex members was confirmed whereas no direct interaction was detected for CHD7-CR1-3-pGBKT7 and the components of the WAR complex (B, E, G). Three biological replicates were performed. Figure modified after Schulz et al. (2014a). 
Considering the fact, that the interaction region could be located outside of the analysed CHD7-CR1-3 fragment, further constructs for CHD7 were generated. Therefore, the whole $\mathrm{CHD} 7$ gene was divided into four overlapping fragments without interrupting any known functional domain (Fig. 9).

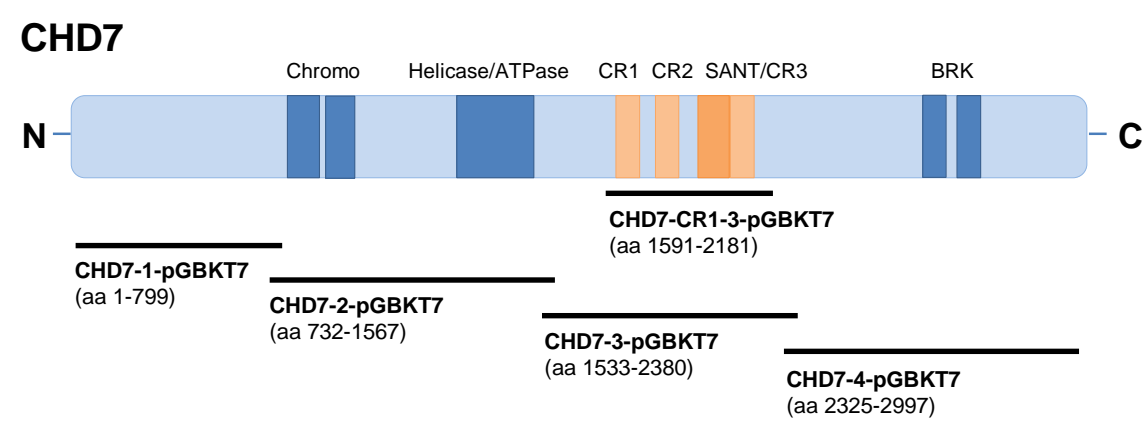

Figure 9: Schematic representation of the CHD7 constructs for yeast two-hybrid experiments.

The ATP-dependent chromatin remodelling enzyme CHD7 belongs to the CHD family and consists of two N-terminal located chromodomains, a helicase/ATPase domain, three conserved regions (CR1CR3), a SANT domain and two BRK domains at the C-terminus. As indicated, the CHD7 gene was divided into four overlapping parts without interrupting any known functional domains (CHD7-1pGBKT7, 1-799 aa; CHD7-2-pGBKT7, 732-1567 aa; CHD7-3-pGBKT7, 1533-2380 aa; CHD7-4pGBKT7, 2325-2997 aa). In addition, the CHD7-CR1-3-pGBKT7 plasmid is shown (Batsukh et al., 2010). Figure modified after Schulz et al. (2014a).

In conclusion, the data from the direct $\mathrm{Y} 2 \mathrm{H}$ experiments revealed no direct interaction of $\mathrm{CHD} 7$ with the WAR complex members and the known direct interaction of CHD8 with the components of the WAR complex was confirmed. At the same time the region which is responsible for the direct interaction was identified to be located within the CHD8 fragment spanning the amino acids 1789-2302, NP_065971.2.

\subsection{Genome-wide expression analysis demonstrates a misregulation of NCC guidance genes in case of CHD7 loss of function}

Already in 1985 Siebert et al. (1985) assumed that the malformations and defects seen in CHARGE syndrome patients result from abnormalities during NCC development. Chd7 knockdown experiments in Xenopus laevis demonstrated that Chd7 is essential for the formation of NCCs (Bajpai et al., 2010). However, only a few Chd7 target genes involved in the formation and migration of NCCs were described (Bajpai et al., 2010). Because nothing is known about the role of Chd7 in 
the guidance of migrating NCCs and the process of EMT, a genome-wide microarray expression analysis was performed with embryos of the Whirligig mouse line at E9.5 at the time of NCCs migration (Schulz et al., 2014b). Animals were kindly provided by K. P. Steel (Sanger Centre, Cambridge, United Kingdom) and the Helmholtz Zentrum, Munich, Germany. The Whirligig mouse line was generated by a largescale ENU mutagenesis programme (Hrabe de Angelis et al., 2000; Hawker et al., 2005). In exon 11 of the $C h d 7$ gene a nonsense mutation (p.W973X) leads to a premature stop codon (Bosman et al., 2005). Heterozygous $\left(C h d 7^{\text {Whil+ }}\right.$ ) mice reveal symptoms which are also present in CHARGE syndrome patients, therefore providing a good model to study features of CHARGE syndrome (Bosman et al., 2005).

The data presented below are published by us (Schulz et al., 2014b). The microarray analysis was performed on four female wild-type $\left(C h d 7^{+/+}\right)$, four heterozygous $\left(C h d 7^{W h i /+}\right)$ and four homozygous $\left(C h d 7^{W h i / W h i}\right)$ mouse embryos. 98 differentially expressed genes showing more than two-fold differences ( $\log 2$ fold-change (FC) $\leq-1$ or $\geq 1$ ) and a false discovery rate (FDR) $<5 \%$ were identified by comparing homozygous $\left(C h d 7^{W h i / W h i}\right)$ and wild-type $\left(C h d 7^{+/+}\right)$embryos. For 71 of these genes the expression was decreased and 27 genes were found with increased expression. The heat map shown in figure 10 displays some of the genes recognised by microarray analysis. Interestingly, many of these differentially expressed genes are known to play a role in NCC migration and axon guidance. Examples are members of the semaphorin family like Sema3a (sema domain, immunoglobulin domain (Ig), short basic domain, secreted, (semaphorin) 3A), Sema3c (sema domain, immunoglobulin domain (Ig), short basic domain, secreted, (semaphorin) 3C) or Sema3d (sema domain, immunoglobulin domain (Ig), short basic domain, secreted, (semaphorin) 3D) which are required for guiding migrating NCCs (Eickholt et al., 1999; Brown et al., 2001; Gitler et al., 2004; Lepore et al., 2006; Sato et al., 2006) and Epha3 (Eph receptor A3), Epha5 (Eph receptor A5) and Epha7 (Eph receptor $A 7)$ belonging to the family of ephrin receptors. Ephrin receptors are also involved in the guidance of NCCs (Kuriyama and Mayor, 2008). Furthermore, transcription factors like Sox10 (SRY (sex determining region Y)-box 10) and Foxd3 (forkhead box D3) which play a role in the early migration process of NCCs were found to be upregulated. A complete list of the 98 differentially expressed genes is shown in table 39 in the appendix. 
Results

\section{Color Key}

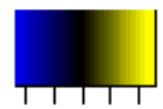

$\begin{array}{lll}-2 & 0 & 2\end{array}$

Row Z-Score
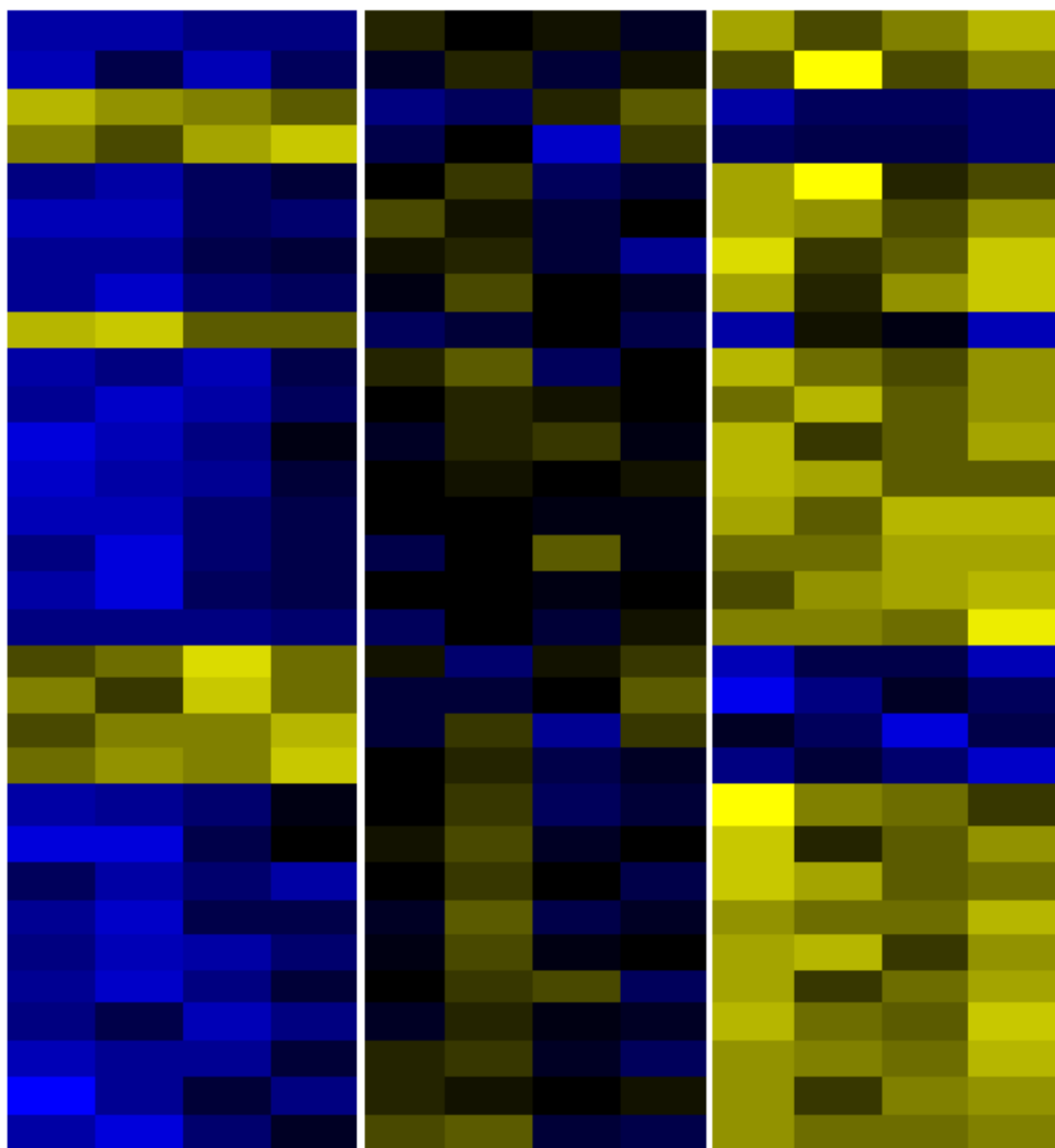

Chd7

Cdh10

Cdh13

Cdh22

Pcdhac2

Pcdh10

Pcdh17

Chl1

Pkp1

Col3a1

Vit

Epha3

Epha7

Sema3a

Sema3c

Sema3d

Bdnf

Sox10

Foxd3

Lmo1

Phf15

Lhx9

Pou3f2

Dach1

Dach2

Alx1

Meox2

Myf6

Myog

Olig3

Prrx1

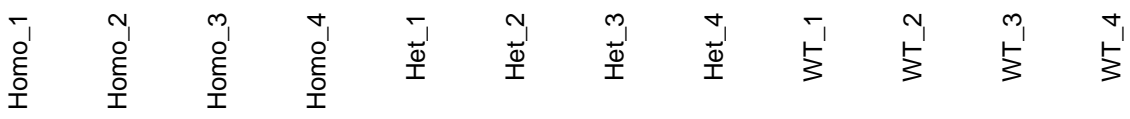

Figure 10: Heatmap of differentially expressed genes found by microarray.

The heatmap shows some of the differentially expressed genes found by comparison of the expression profile of four female mouse embryos (E9.5) per genotype (wild-type $\left(C h d 7^{+/ /+}\right.$): WT 1-4, heterozygous $\left(C h d 7^{W h i /+}\right)$ : Het 1-4 and homozygous $\left(C h d 7^{W h i / W h i}\right)$ : Homo 1-4). The results illustrate a regulating function of CHD7 of genes involved in NCC and axon guidance and transcription factors.

Figure modified after Schulz et al. (2014b). 


\subsection{Expression analysis by RT-qPCR confirms the results of the microarray}

To validate the data of the genome-wide microarray analysis, the expression of genes involved in NCC guidance, namely Sema3a, Sema3d, Epha3, Sox10 and the two further genes Trp53bp2 (transformation related protein 53 binding protein 2) and Gfra2 (glial cell line derived neurotrophic factor family receptor alpha 2) were analysed by RT-qPCR. Furthermore, these genes were chosen to have a complete validation of upregulated, nonregulated and downregulated genes. The relative expression of these genes is shown in figure 11 and published by us (Schulz et al., 2014b). The same RNA used for the microarray analysis was employed for the RTqPCR. Four biological replicates for heterozygous $\left(C h d 7^{W h i /+}\right)$ and homozygous $\left(C h d 7^{W h i / W h i}\right)$ mice were analysed. Four wild-type $\left(C h d 7^{+/+}\right)$embryos were pooled and used as a calibrator. Data were normalised against three reference genes (Tbp (TATA box binding protein), Sdha (Succinate dehydrogenase complex, subunit A) and Hprt (Hypoxanthin-phosphoribosyl-transferase)). The microarray expression analysis revealed a clear upregulation of Sema3a, Sema3d and Epha3. The results of the RT-qPCR showed that in heterozygous $\left(C h d 7^{\text {Whil+ }}\right)$ animals the relative expression of Sema3a was decreased up to $50 \%$. Homozygous $\left(C h d 7^{\text {Whi/Whi }}\right)$ mice showed a reduction of Sema3a expression between 60-70\%. A similar decrease in expression was observed for Sema3d. The relative expression of Epha3 was reduced more than $50 \%$ in heterozygous $\left(C h d 7^{W h i /+}\right)$ embryos and 60-80\% in homozygous $\left(C h d 7^{\text {Whi/Whi}}\right)$ embryos. The average relative expression of Trp53bp2 remained mostly unchanged in heterozygous $\left(C h d 7^{\text {Whi/+ }}\right)$ and homozygous $\left(C h d 7^{\text {Whi/Whi }}\right)$ embryos compared to wild-type $\left(C h d 7^{+/+}\right)$. According to the microarray data the expression of the transcription factor Sox 10 and Gfra2 was upregulated (appendix, Tab. 39). The relative expression of Sox10 measured by RT-qPCR was increased about $35 \%$ in heterozygous $\left(C h d 7^{W h i /+}\right)$ mice. The increase in expression of Sox10 was even stronger in the homozygous $\left(C h d 7^{W h i / W h i}\right)$ stage where in half of the embryos Sox10 was double as high as in wild-type $\left(C h d 7^{+/+}\right)$embryos. The Gfra2 expression was in half of the heterozygous $\left(C h d 7^{\text {Whi/+ }}\right)$ embryos 0.7-2.3 times higher

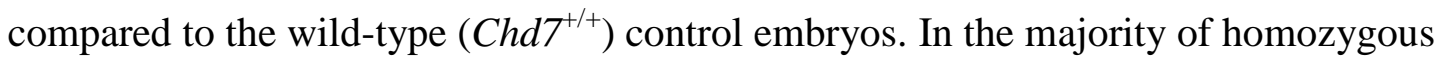
$\left(C h d 7^{W h i / W h i}\right)$ embryos the Gfra2 expression was markedly more than double as the 
expression in the wild-type $\left(C h d 7^{+/+}\right)$embryos. Altogether, the results of the RTqPCR reflect the data of the genome-wide microarray analysis.
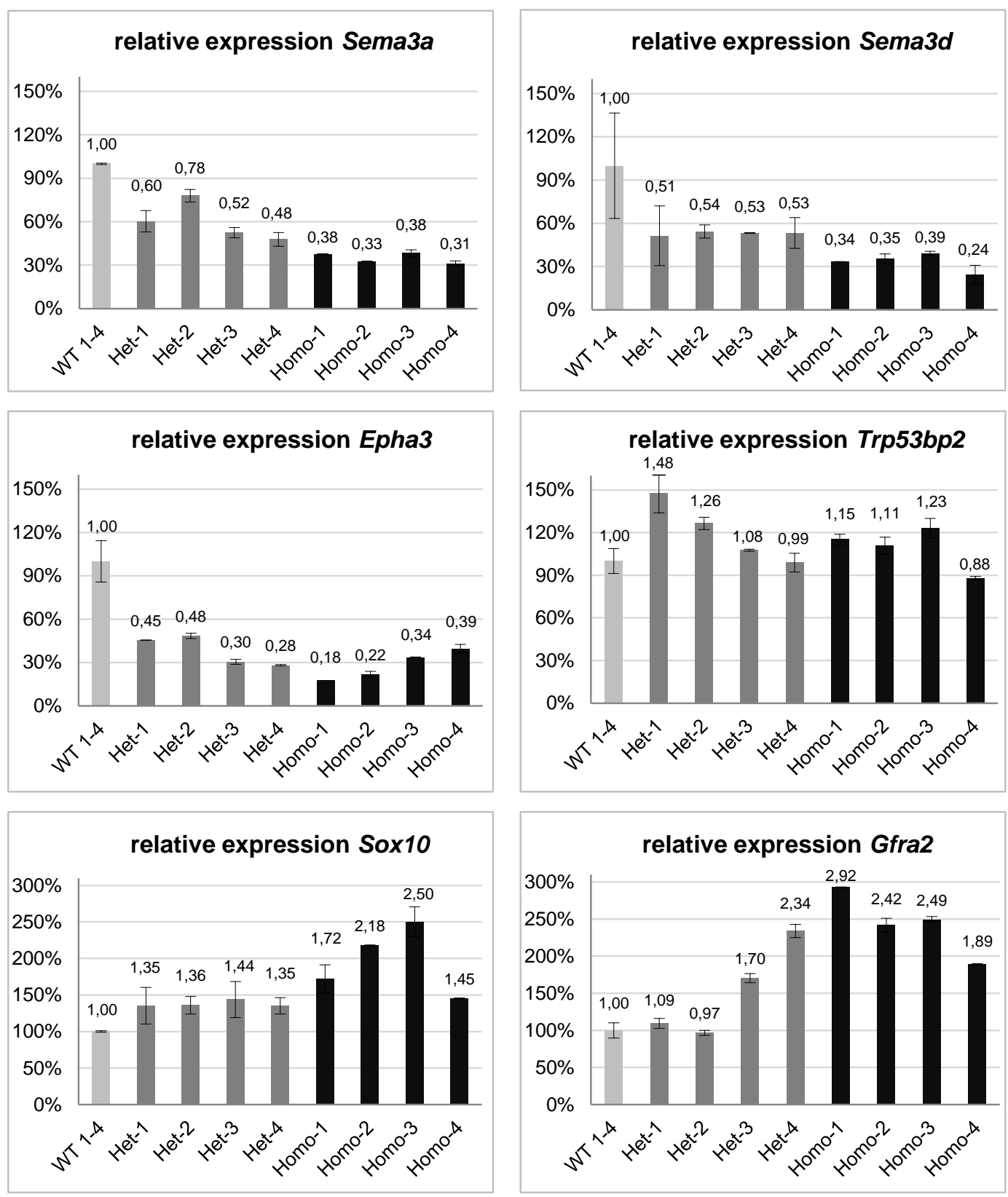

Figure 11: Results of the gene expression analysis by RT-qPCR.

A selection of six genes (Sema3a, Sema3d, Epha3, Trp53bp2, Sox10 and Gfra2) was used to validate the microarray data by RT-qPCR. The same RNA from female embryos of the Whirligig mouse line was used as for the microarray. The experiment was performed on four biological replicates per genotype $\left(C h d 7^{W h i /+}\right.$ and $\left.C h d 7^{W h i / W h i}\right)$. The RNA from the four wild-type $\left(C h d 7^{+/+}\right)$embryos was pooled and utilised as a calibrator. The data were normalised against the three reference genes $T b p$, Sdha and Hprt. The expression of Sema3a, Sema3d and Epha3 is downregulated, Trp53bp2 is mostly unaffected and Sox10 and Grfa2 are upregulated. These results confirm the data from the wholegenome microarray analysis. Figure modified after Schulz et al. (2014b). 


\subsection{There is no gender specific effect on the expression of Sema3a, Sema3d, Epha3, Trp53bp2, Sox10 and Gfra2 in Whirligig mouse embryos}

To further validate whether the data from the microarray and the RT-qPCR are female specific, the same genes were analysed by another RT-qPCR approach using RNA extracted from male wild-type $\left(C h d 7^{+/+}\right)$, heterozygous $\left(C h d 7^{\text {Whil+}}\right)$ and homozygous $\left(C h d 7^{\text {Whi/Whi }}\right.$ ) mouse embryos (E9.5). The results described below are published by us (Schulz et al., 2014b). Three biological replicates for each genotype were analysed. The three wild-type $\left(C h d 7^{+/+}\right)$embryos were pooled and served as a calibrator. The data were normalised against the reference genes Tbp, Sdha and Hprt. The results are represented in figure 12. The relative expression of Sema3a was downregulated in all embryos. Homozygous $\left(C h d 7^{\text {Whi/Whi}}\right)$ mice showed with a decrease of more than $50 \%$ up to $70 \%$ the most precise effect. In the majority of heterozygous $\left(C h d 7^{W h i /+}\right)$ individuals Sema3d expression was about half reduced compared to wild-type $\left(C h d 7^{+/+}\right)$embryos. The Sema3d expression was even more decreased $(>70 \%)$ in homozygous $\left(C h d 7^{W h i / W h i}\right)$ mice. Epha3 was also found to be downregulated in the majority of heterozygous $\left(C h d 7^{\text {Whil+ }}\right)$ and homozygous $\left(C h d 7^{W h i / W h i}\right)$ embryos with about $70 \%$. While the expression of Trp53bp2 remained unaffected, the expression of Sox10 and Grfa2 was increased. Especially homozygous $\left(C h d 7^{\text {Whi/Whi }}\right)$ mice showed a three times higher expression level of Gfra2 than wild-type $\left(C h d 7^{+/+}\right)$embryos. In summary, it can be stated that female and male embryos showed a similar expression of the analysed genes which corresponds to the results of the microarray. 

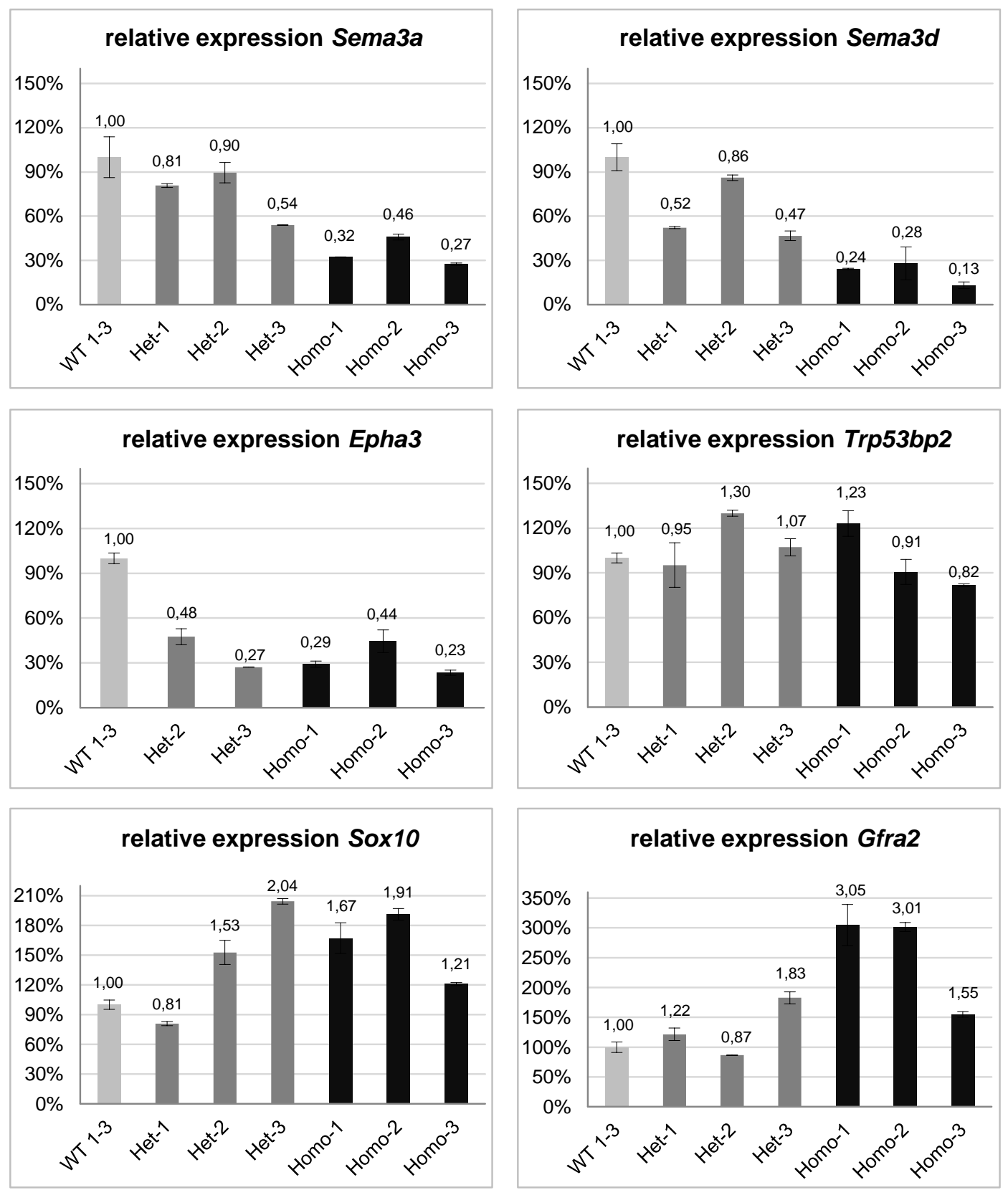

Figure 12: Repetition of RT-qPCR.

RNA from male E9.5 embryos was used. Three biological replicates were performed for each genotype $\left(C h d 7^{W h i /+}\right.$ and $\left.C h d 7^{W h i / W h i}\right)$. Pooled RNA from three wild-type $\left(C h d 7^{+/+}\right)$embryos served as a calibrator. Data were normalised against the three reference genes Tbp, Sdha and Hprt. The same genes were analysed under identical conditions as described before (Fig. 11). The Sema3a, Sema3d and Epha3 expression is found to be downregulated. Trp53bp2 expression is mostly unaffected and the Sox10 and Grfa2 expression is upregulated, demonstrating that there is no gender specific CHD7 regulation of the analysed genes. One of the biological replicates of the heterozygous $\left(C h d 7^{\text {Whil+t}}\right)$ mouse embryos analysing the Epha3 expression was excluded due to technical issues. Figure modified after Schulz et al. (2014b). 


\subsection{CHD7 and SEMA3D show a similar expression pattern in mouse embryos}

The data from the genome-wide microarray analysis revealed a high number of differentially expressed genes which play a role in NCC migration (Schulz et al., 2014b). It was shown that CHD7 has a regulatory effect on some members of the semaphorin family (Fig. 10) (Schulz et al., 2014b). Therefore, the expression pattern of CHD7 and SEMA3D was analysed on paraffin sections of wild-type mouse embryos (E12.5) and compared with each other. Figure 13 shows three serial made sagittal sections from the embryo (Fig. 13A, B, C) after immunohistochemistry (DAB staining). Picture A shows the negative control, picture B represents the expression pattern of CHD7 and the expression profile of SEMA3D can be seen in picture C. CHD7 is expressed mainly in the brain, heart, vertebrae and nerves along the spine and the sensory nerve ends above the eye. SEMA3D is also expressed in the brain, heart and different structures of the nervous system like CHD7. While CHD7 seems to be more restricted to a specific area, SEMA3D is expressed also in the surrounding epithelium. Unfortunately, further investigation of SEMA3D and CHD7 expression in wild-type $\left(C h d 7^{+/+}\right)$and heterozygous $\left(C h d 7^{W h i /+}\right)$ mouse embryos of the Whirligig mouse line failed as well as the expression analysis of SEMA3A due to technical issues affecting the colour reaction. Several repetitions with different mouse embryos were performed, solutions, such as paraffin, fixating solution and the DAB staining solutions were exchanged but the technical problem could not be solved. It would be interesting to analyse the changes in the expression pattern of semaphorins in CHD7 deficient mouse embryos. 

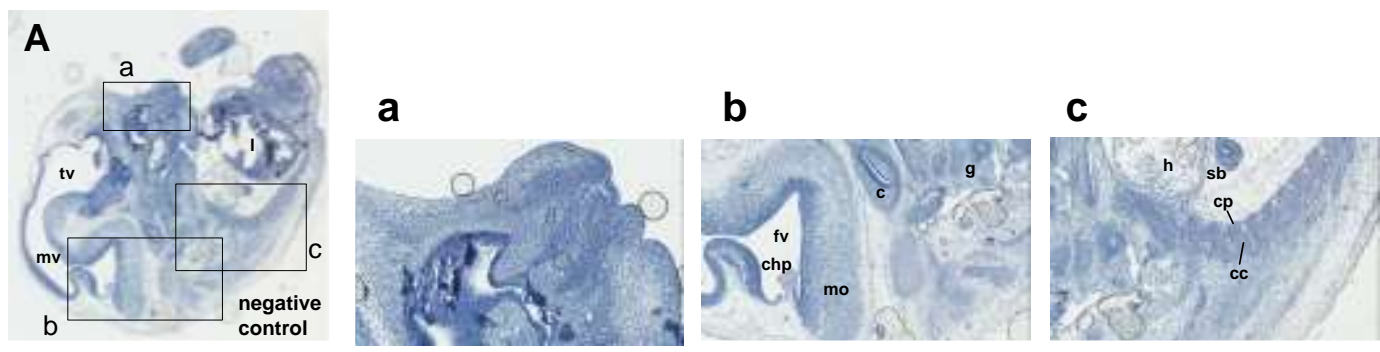

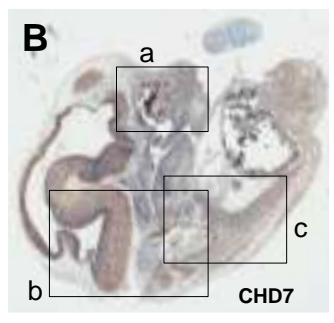

\section{a}

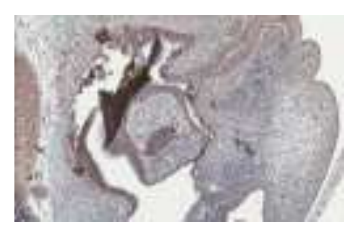

b

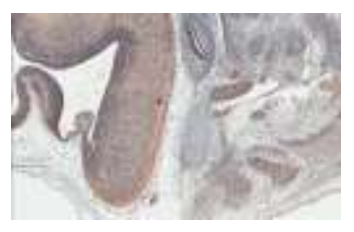

C
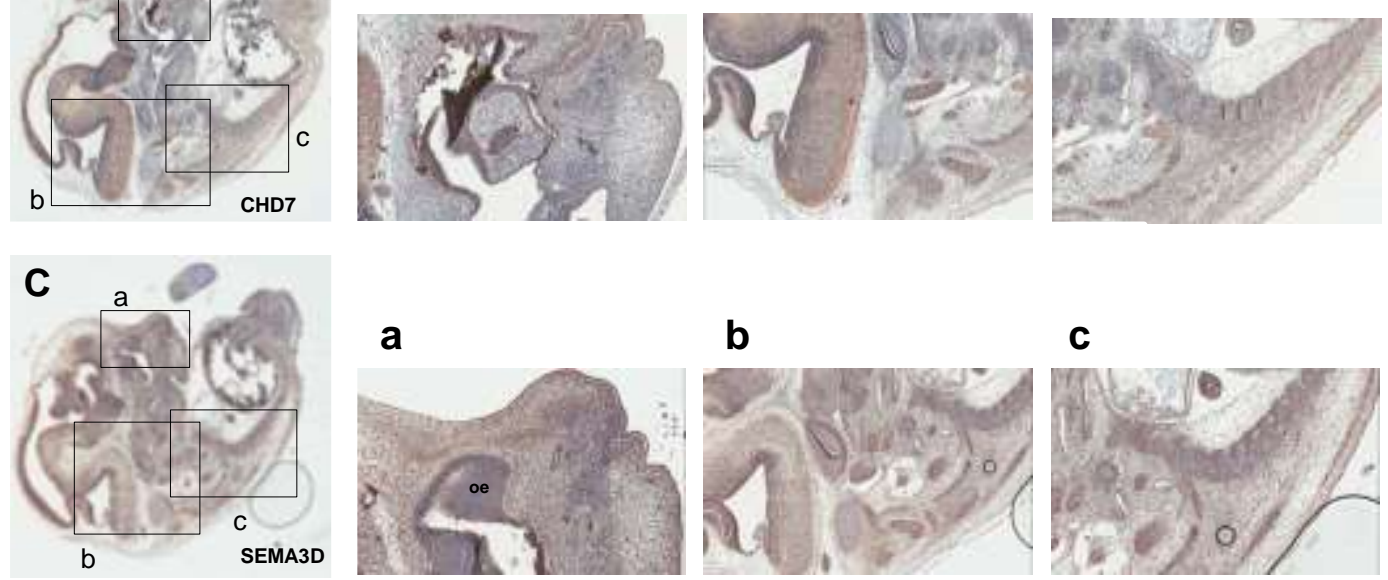

a

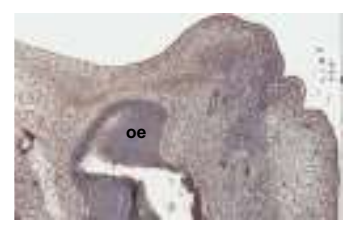

b

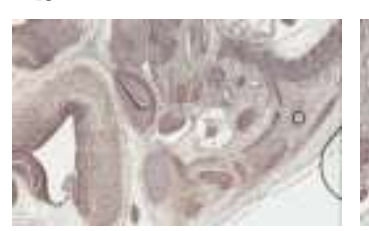

C

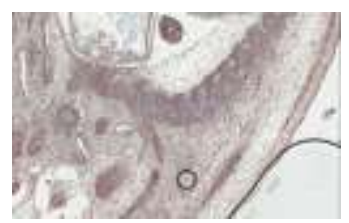

Figure 13: CHD7 and SEMA3D expression analysis using paraffin sections of mouse embryos stained with DAB.

Serial sagittal paraffin sections were prepared using wild-type mouse embryos (E12.5). The expression pattern of CHD7 (B) and SEMA3D (C) were analysed by DAB staining. No primary antibody was used for the negative control (A). Different regions of the embryos are shown in higher magnification: front head (a), neck (b) and dorsum (c). The negative control showed no unspecific staining, indicating that the universal secondary antibody does not bind unspecifically to the tissue of the embryos (A). CHD7 expression was found in the medulla oblongata, choroid plexus differentiating from roof of fourth ventricle, cochlea, heart, cartilage primordium of body of vertebra and in different ganglia e.g. along the spine and above the eye (B). The SEMA3D expression pattern resembles the expression of $\mathrm{CHD} 7$ in the medulla oblongata, choroid plexus differentiating from roof of fourth ventricle, cochlea, heart, cartilage primordium of body of vertebra and in different ganglia (C). $\mathrm{c}=$ cochlea, $\mathrm{cc}=$ cartilage condensation being primordium of vertebral body, $\mathrm{chp}=$ choroid plexus differentiating from roof of fourth ventricle, $\mathrm{cp}=$ cartilage primordium of body of vertebra, $\mathrm{fv}$ $=$ forth ventricle, $\mathrm{g}=$ ganglia, $\mathrm{h}=$ heart, $1=$ liver, $\mathrm{mo}=$ medulla oblongata, $\mathrm{mv}=$ mesencephalic vesicle, oe $=$ olfactory epithelium, $\mathrm{sb}=$ segmental bronchus within accessory lobe of right lung, $\mathrm{tv}=$ right telencephalic vesicle. 


\subsection{Results of $S E M A 3 A$ and $S E M A 3 D$ mutation screens in patients with CHARGE syndrome}

CHARGE syndrome is a phenotypically heterogeneous disorder, so it is possible that there are other genes which might act as modifiers leading to a more severe phenotype when they are mutated. In 5-10 \% of typical CHARGE patients and 40$60 \%$ of patients suspected of having CHARGE the underlying cause remains unknown (Janssen et al., 2012) so it could be possible that other "CHARGE genes" exist. For that reason 45 CHARGE syndrome patients without a mutation in the CHD7 gene were screened for mutations in SEMA3A (GenBank accession number: NM_006080.2) and SEMA3D (GenBank accession number: NM_152754.2). All 17 coding exons and flanking intronic sequences of SEMA3A and SEMA3D were analysed by amplifying these regions by PCR and screening for mutations by sequencing. The results from the SEMA3A mutation screen are published by us (Schulz et al., 2014b). For SEMA3A three non-synonymous variants were found: c.196C>T (p.R66W), c.2002A>G (p.I668V) and c.2062A>G (p.T688A). Sequences are shown in figure 14. The variants are indicated by red arrows. The variant p.R66W was located in the sema domain, a conserved module of ligand-receptor interaction, also present in other proteins like plexins (Antipenko et al., 2003) and was predicted to be damaging (Polyphen 2, SIFT and mutation taster). By screening the parents of the patient carrying the c.196C>T (p.R66W) variant it was shown that this variant was inherited from the healthy father. The two other identified variants p.I668V and p.T688A were predicted to be benign (Polyphen 2). The parents were not available for a further clarification. 
SEMA3A, exon 2 c.196C >T/p.R66W

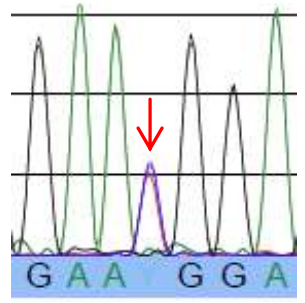

forward sequence

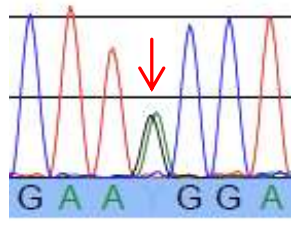

reverse sequence
SEMA3A, exon 17

c.2002A>G/p. $1668 \mathrm{~V}$

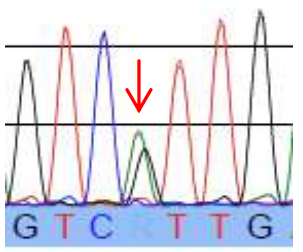

forward sequence

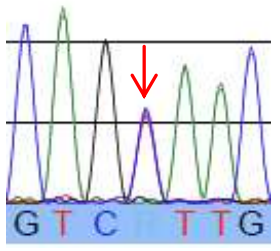

reverse sequence
SEMA3A, exon 17

c.2062A>G/p.T688A

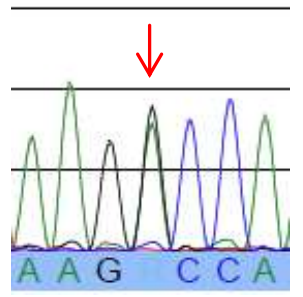

forward sequence

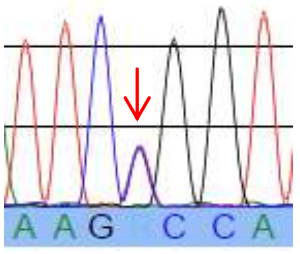

reverse sequence

Figure 14: Results of the SEMA3A mutation screen.

All coding exons of the SEMA3A gene were sequenced in 45 CHD7 negative patients diagnosed with CHARGE syndrome. Three non-synonymous variants were identified: c.196C >T (p.R66W), c.2002A $>$ G (p.I668V) and c.2062A $>$ G (p.T688A). The mutations are indicated by red arrows in the forward and reverse sequence, respectively.

Sequencing of SEMA3D revealed following non-synonymous variants: c.193T $>$ C (p.S65P), c.1272C>A (p.H424Q) and c.2101A>C (p.K701Q) (Fig. 15). While the non-synonymous SNP p.K701Q is a common variant with an allele frequency of 0.346 in the ESP cohort population (SNPdev, n.d.), the variant p.S65P is less common. In the ESP cohort population it has an allele frequency of 0.017 (SNPdev, n.d.). Both variants are predicted to be benign (Polyphen and Polyphen 2). The variant p.H424Q is quite rare. It was observed in the ESP cohort population with an allele frequency of 0.002 (SNPdev, n.d.) and it was found to be located in a highly conserved domain. In silico programms predicted the variant p.H424Q as a protein "damaging" variant (Polyphen, Polyphen 2, SIFT). For further clarification the parents of the patients were screened. It was shown that the variant p.H424Q was inherited from the healthy father.

Furthermore, in ten CHD7 negative CHARGE patients all 17 coding exons of SEMA3A and SEMA3D were analysed for copy number defects (large deletions and duplications) by qPCRs. In these patients copy number defects were excluded. 
SEMA3D, exon2

c.193T>C/p.S65P

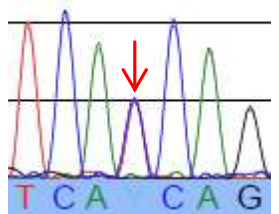

forward sequence

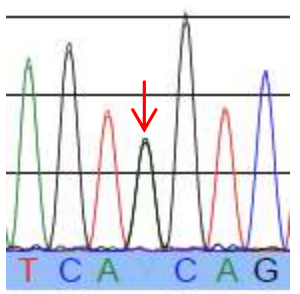

reverse sequence
SEMA3D, exon11

c.1272C $>\mathrm{A} / \mathrm{p} . \mathrm{H} 424 \mathrm{Q}$

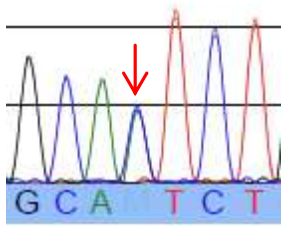

forward sequence

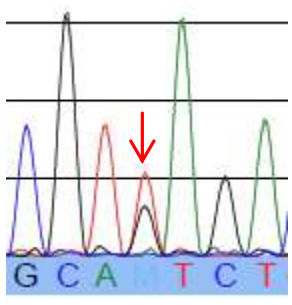

reverse sequence
SEMA3D, exon17

c. $2101 \mathrm{~A}>\mathrm{C} / \mathrm{p} . \mathrm{K} 701 \mathrm{Q}$

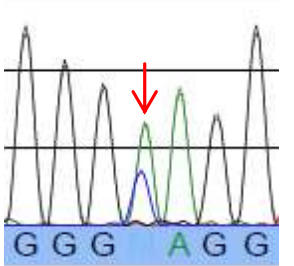

forward sequence

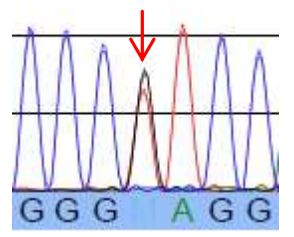

reverse sequence

Figure 15: Results of the $S E M A 3 D$ mutation screen.

The 17 exons of the SEMA3D gene were sequenced in 45 CHD7 negative CHARGE patients. Three non-synonymous variants were found: c.193T>C (p.S65P), c.1272C>A (p.H424Q) and c.2101A>C (p.K701Q). The mutations are shown in the forward and reverse sequence indicated by red arrows.

\subsection{Knockdown of $\mathrm{Chd} 7$ causes alteration in sema3a expression in Xenopus laevis}

Chd7 is highly conserved throughout different species (Bosman et al., 2005; Bajpai et al., 2010; Schnetz et al., 2010). It was shown that Chd7 deficient Xenopus laevis embryos mimic basic features of CHARGE syndrome (Bajpai et al., 2010). To study the effect of Chd7 on the expression of sema3a in another species, Chd7 was downregulated in Xenopus laevis mediated by Chd7 MO. The data below are published by us (Schulz et al., 2014b). The sema3a expression pattern was analysed by WMISH at the neurula stage 20 (Fig. 16) and the tailbud stage 27 (Fig. 17). Embryos were injected with a MO in combination with lacZ RNA as a lineage tracer in one blastomere at a two-cell stage. The injected side is indicated by the blue lac $Z$ staining (Fig. 16A-F; Fig. 17B, D, F). Control embryos were injected with $20 \mathrm{ng}$ control MO (Co MO) which has no target RNA. Embryos injected with the Co MO showed as expected a normal sema3a expression pattern in the midbrain-hindbrain boundary (mh) (Fig. 16A; Fig. 17A, B) and somites (s) (Fig. 16B; Fig. 17A, B). Tailbud stages showed an additional expression in the splanchic mesoderm (sm) 
(Fig. 17A, B). After Chd7 was downregulated by injecting $10 \mathrm{ng}$ Chd7 MO, in about $70 \%$ of the embryos a reduction of sema3a expression were detected. Approximately $60 \%$ of these embryos showed a severe downregulation of the sema3a expression pattern in general or the expression in one district region was completely missing (Fig. 16C, D; Fig. 17D). After injecting $20 \mathrm{ng} \mathrm{Chd} 7 \mathrm{MO}$, in almost all analysed embryos of the neurula and tailbud stage the sema3a expression was dramatically decreased or completely absent (Fig. 16E, F; Fig. 17F).
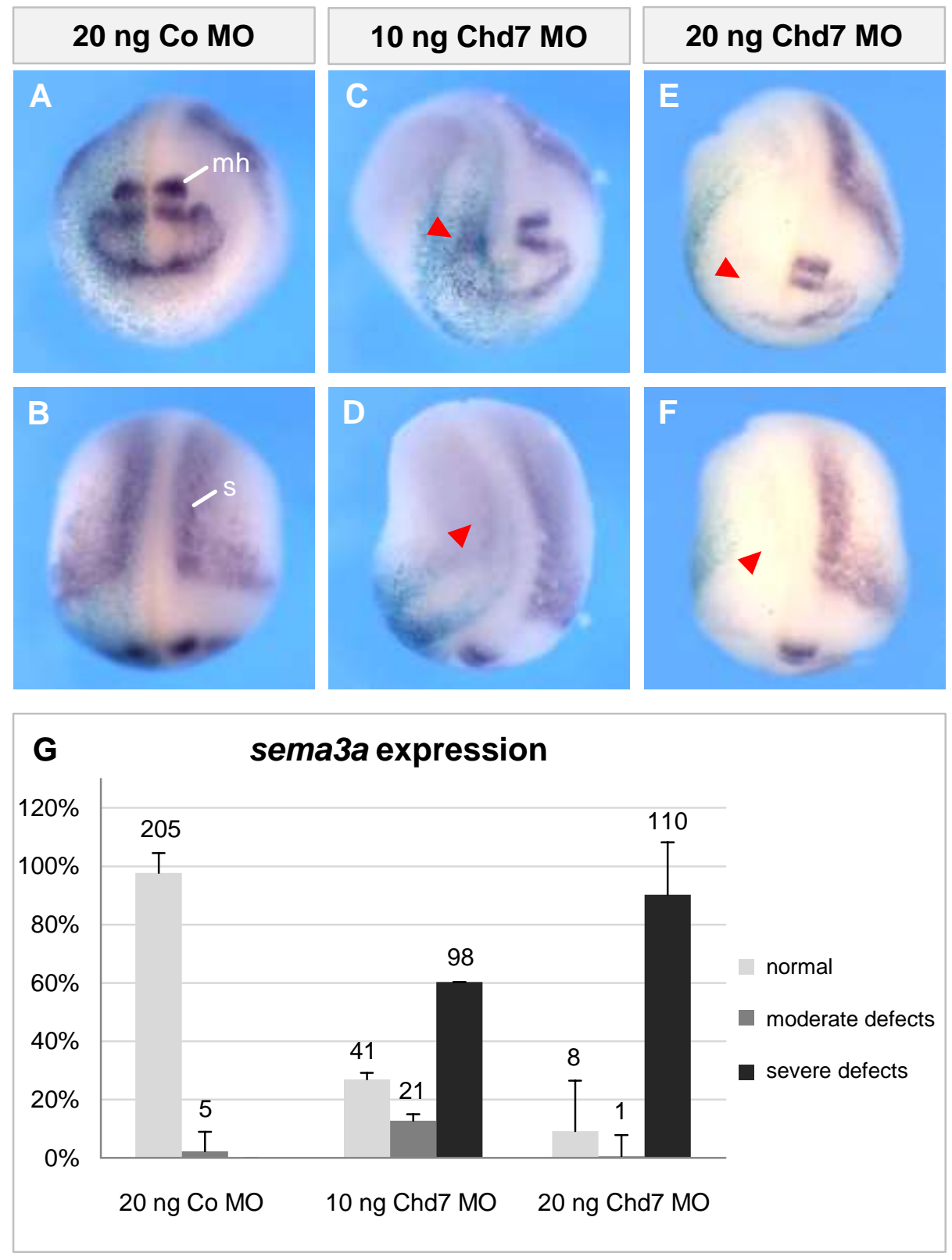

Figure 16: Sema3a expression analysis by whole mount in situ hybridisation after Chd7 knockdown in Xenopus laevis (neurula stage 20).

One blastomere of the two-cell stage embryos was injected with a MO and lacZ RNA as a lineage tracer. Embryos were injected with $20 \mathrm{ng}$ control MO (A, B), $10 \mathrm{ng}$ Chd7 MO (C, D) or 20 ng CHD7 


\section{Results}

MO (E, F). The injected side of the embryo is indicated by a blue lac $Z$ staining. The expression pattern of sema3a (purple staining) was analysed. Nearly all control embryos showed a normal expression pattern of sema3a (A, B); midbrain hindbrain boundary (mh); somites (s). After knocking down Chd7 with $10 \mathrm{ng}$ Chd7 MO, in about $60 \%$ of the embryos a clear reduced sema $3 a$ expression in the somites and the head region was observed $(\mathbf{C}, \mathbf{D})$. An injection of $20 \mathrm{ng}$ Chd7 MO led to a complete loss of sema3a expression or to a drastic reduced expression pattern in $90 \%$ of the embryos $(\mathbf{E}, \mathbf{F})$. The graph summarises three independent experiments $(\mathbf{G})$. The diagram reflects the percentage of the embryos showing a normal sema3a expression pattern (light grey columns), moderate (grey columns) or severe defects (black columns). Standard error of the means is shown. On top of each column the total number of the embryos counted in the different categories is listed. Figure modified after Schulz et al. (2014b).

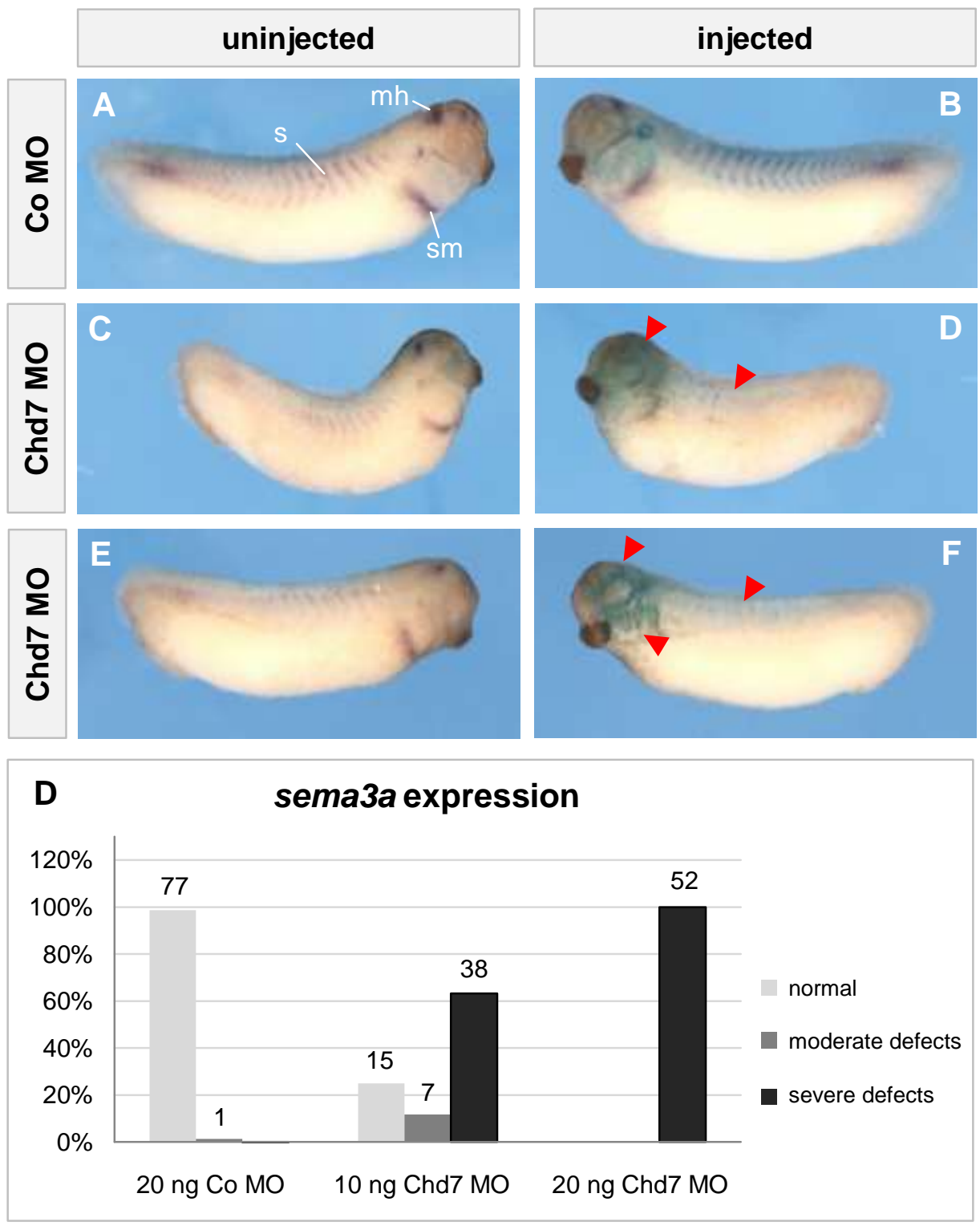

Figure 17: Sema3a expression after Chd7 knockdown in Xenopus laevis (tailbud stage 27).

One blastomere of two-cell embryos was injected with a MO (20 ng control MO (A, B), 10 ng Chd7 MO (C, D) or $20 \mathrm{ng}$ CHD7 MO (E, F)) in combination with lacZ RNA for lineage tracing. A blue 
lacZ staining indicates the injected side of the embryo $(\mathbf{B}, \mathbf{D}, \mathbf{F})$. The expression pattern of sema3a (purple staining) was analysed by whole mount in situ hybridisation. In $99 \%$ of the control embryos the expression pattern of sema3a remained unchanged $(\mathbf{A}, \mathbf{B})$; midbrain hindbrain boundary $(\mathrm{mh})$; somites (s); splanchic mesoderm (sm). After the knockdown of Chd7 with $10 \mathrm{ng}$ Chd7 MO $60 \%$ of the embryos showed a reduced sema3a expression in the somites and the head region (C, D). A complete loss of sema3a expression or a drastic reduced expression was observed in all embryos after $20 \mathrm{ng}$ Chd7 MO injections (E, F). The columns of the diagram show the percentage of embryos with normal sema3a expression (light grey), moderate (grey) or severe changes in the expression pattern (black). On top of each column the amount of embryos obtained in the different categories are listed. The data refer to one biological replicate. Figure modified after Schulz et al. (2014b).

\subsection{The Chd7 MO phenotype was successfully rescued by human $\mathrm{CHD} 7$ RNA}

Rescue experiments were performed to check whether human $C H D 7$ RNA is able to rescue the Chd7 MO phenotype. The human CHD7 full-length plasmid was kindly provided by J. Wysocka (Department of Developmental Biology, Stanford University School of Medicine, Stanford, California). The following data are published by us (Schulz et al., 2014b). CHD7 DNA was transcribed into sense RNA to obtain the rescue construct (hCHD7 RNA). Xenopus laevis embryos were injected as described before. A WMISH was performed at neurula stages 20 and 21 to analyse the expression of sema3a. Embryos injected with $\mathrm{Co} \mathrm{MO}$ and the rescue construct and uninjected embryos were used as controls. More than $95 \%$ of these embryos showed a normal sema3a expression (Fig. 18A-D; Fig. 19A-D). After injecting $10 \mathrm{ng}$ Chd7 MO, half of the embryos showed a massively reduced sema3a expression and more than $30 \%$ a moderate reduction (Fig. 18E, F). For the rescue experiments embryos were injected with $10 \mathrm{ng}$ of $\mathrm{Chd} 7 \mathrm{MO}$ to knockdown Chd7 in combination with $1 \mathrm{ng}$ of the rescue construct hCHD7 RNA which is not recognised by the MO. The embryos of the Chd7 knockdown were compared with the embryos after the rescue. The percentage of embryos showing a severe reduction of sema3a expression was reduced from $53 \%$ down to $6 \%$. At the same time the percentage of embryos with a normal expression pattern was increased from $13 \%$ to $52 \%$ (Fig. 18I). The rescue of the Chd7 MO phenotype was slightly more effective after injecting $1.5 \mathrm{ng}$ hCHD7 RNA (Fig. 19I). The percentage of severe alterations in sema3a expression was decreased from $61 \%$ to $4 \%$ and the percentage of embryos showing a normal phenotype was increased from $24 \%$ to $56 \%$. 
Results
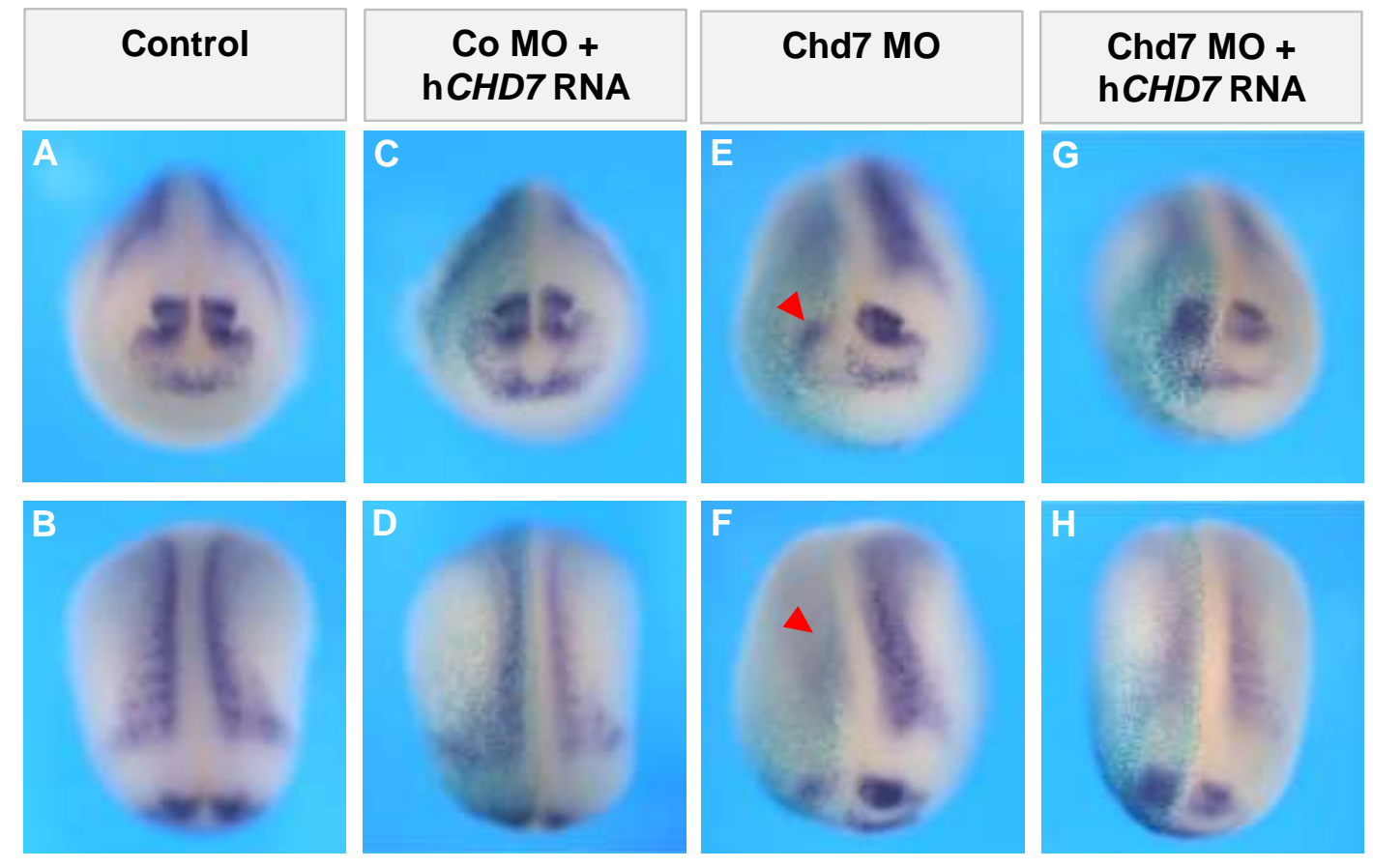

I

sema3a expression

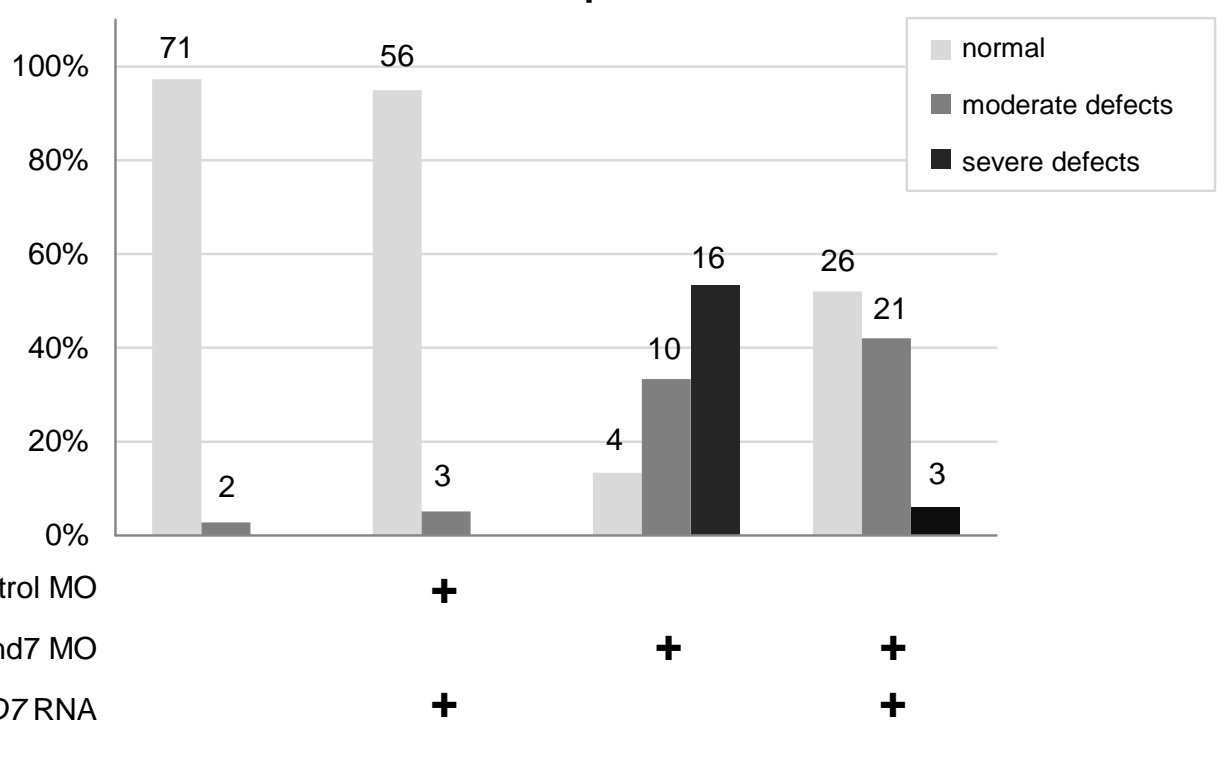

Figure 18: Rescue of the Chd7 MO phenotype in Xenopus laevis (neurula stage 21).

As a rescue construct human CHD7 RNA (hCHD7 RNA) was used which is not recognised by the Chd7 MO. Embryos were injected (one blastomere of a two-cell stage) with either $10 \mathrm{ng}$ of a Co MO and $1 \mathrm{ng} \mathrm{hCHD7} \mathrm{RNA} \mathrm{for} \mathrm{the} \mathrm{rescue} \mathrm{(C,} \mathrm{D),} 10 \mathrm{ng}$ Chd7 MO (E, F) or $10 \mathrm{ng}$ Chd7 MO and $1 \mathrm{ng}$ hCHD7 RNA (G, H). All embryos were co-injected with lacZ RNA as lineage tracer. By whole mount in situ hybridisation the sema3a expression was analysed (purple staining). Uninjected embryos were used as a control. Nearly all uninjected embryos showed the normal sema3a expression pattern (A, B). In more than $90 \%$ of the embryos co-injected with a Co MO and hCHD7 RNA the sema3a expression pattern remained unchanged $(\mathbf{C}, \mathbf{D})$. After downregulation of Chd7 by injecting $10 \mathrm{ng}$ Chd7 MO, more than $50 \%$ of the embryos showed severe defects, meaning a clear reduced sema $3 a$ expression pattern and/or missing sema $3 a$ expression in either somites or head region $(\mathbf{E}, \mathbf{F})$. 


\section{Results}

In more than $30 \%$ of these embryos moderate reduction of sema3a was detected and in about $10 \%$ of the embryos the sema3a expression pattern remained unaltered. More than half of the embryos coinjected with $10 \mathrm{ng}$ Chd7 MO to knockdown Chd7 and $1 \mathrm{ng}$ hCHD7 RNA to rescue the Chd7 MO phenotype showed a normal sema3a expression pattern $(\mathbf{G}, \mathbf{H})$ and about $40 \%$ had a moderate reduced sema3a expression pattern. (I) Graph summarises the percentage of embryos obtained from one biological replicate with normal sema3a expression (light grey columns), moderate (grey columns) or severe changes in the expression pattern (black columns). Numbers on top of each column represent the amount of embryos counted in the different categories. Figure modified after Schulz et al. (2014b).

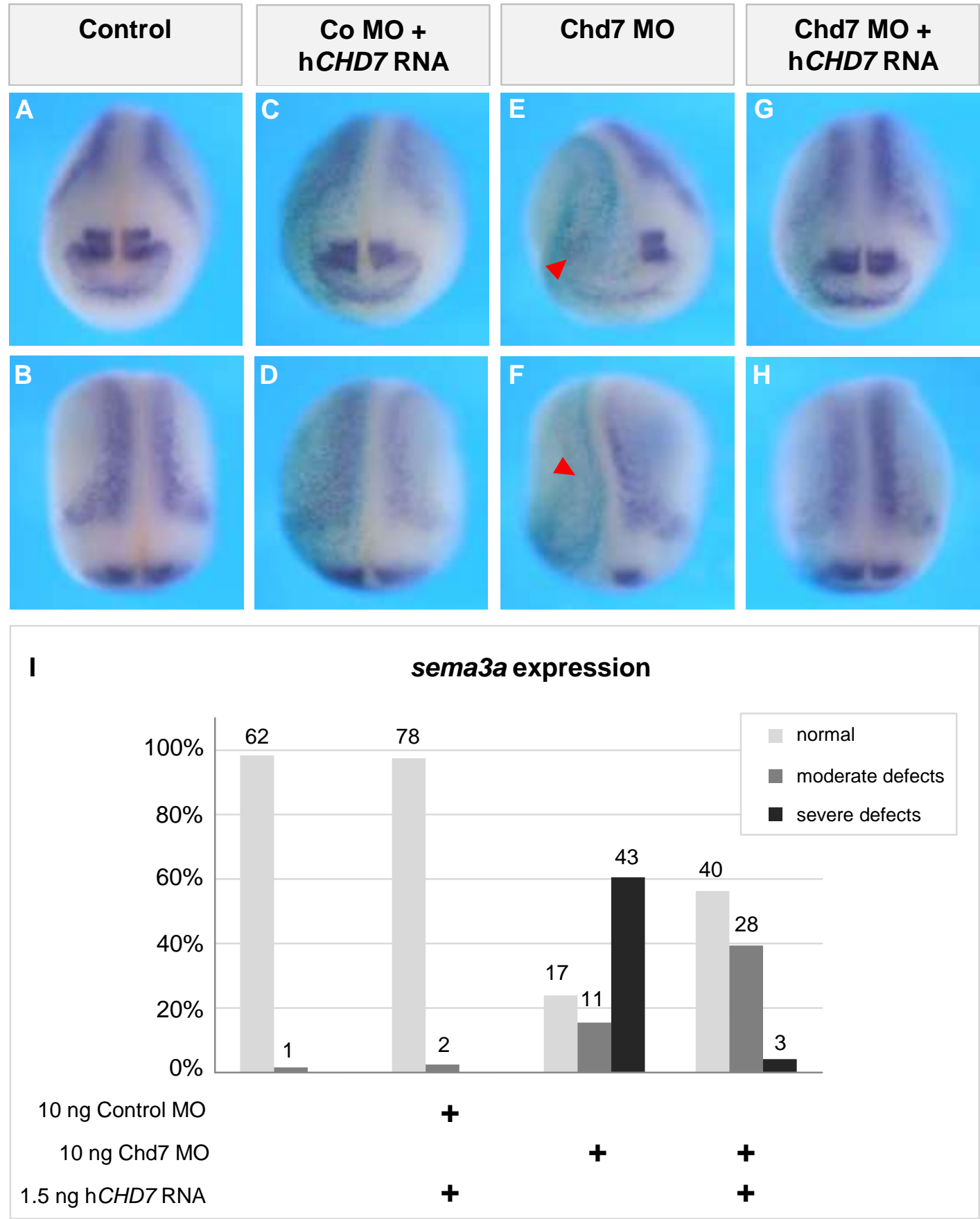


Figure 19: Repetition of the rescue of the Chd7 MO phenotype in Xenopus laevis (neurula stage 20).

Single blastomeres of two-cell stages were injected with either $10 \mathrm{ng}$ of a control MO and $1.5 \mathrm{ng}$ human rescue CHD7 RNA (hCHD7 RNA) (C, D), $10 \mathrm{ng}$ Chd7 MO (E, F) or $10 \mathrm{ng}$ Chd7 MO and $1.5 \mathrm{ng}$ hCHD7 RNA $(\mathbf{G}, \mathbf{H})$. Co-injection of lacZ RNA served as a lineage tracer. Sema3a expression was analysed by whole mount in situ hybridisation (purple staining). Nearly all uninjected control embryos showed a normal sema3a expression pattern (A, B). In more than $95 \%$ of the embryos coinjected with a Co MO and hCHD7 RNA the sema3a expression pattern remained unchanged (C, D). After injection of $10 \mathrm{ng}$ Chd7 MO, more than $60 \%$ of the embryos showed severe changes in the sema3a expression pattern $(\mathbf{E}, \mathbf{F})$. After co-injection of $10 \mathrm{ng}$ Chd7 MO to knockdown Chd7 and $1.5 \mathrm{ng}$ hCHD7 RNA to rescue the Chd7 MO phenotype, in about $60 \%$ of the embryos a normal sema3a expression pattern was observed $(\mathbf{G}, \mathbf{H})$ and $40 \%$ showed moderate changes in the sema3a expression pattern. (I) The graph summarises the data obtained from one biological replicate. Columns represent the percentage of embryos with normal sema3a expression (light grey), moderate (grey) or severe reduction of the expression pattern (black). Numbers on top of each column indicate the amount of embryos counted in the different categories. Figure modified after Schulz et al. (2014b).

\subsection{Knockdown of Sema3a and Sema3d causes migration defects of NCCs in Xenopus laevis}

It was shown in Xenopus laevis embryos that a MO mediated downregulation of Chd7 leads to migration defects of NCCs (Bajpai et al., 2010). Semaphorins are involved in the process of NCC guidance (Kuriyama and Mayor, 2008). Because Sema3a and Sema3d were found to be downregulated in CHD7 deficient mice (Schulz et al., 2014b), these genes were chosen for knockdown experiments in Xenopus laevis to analyse the effect on NCC migration. Embryos were injected in one blastomere of a two-cell stage. The expression of the NCC marker twist was analysed by WMISH at the neurula stage 21 in three independent experiments (Fig. 20) and the tailbud stage 26 in two independent experiments (Fig. 21). Control embryos injected with $20 \mathrm{ng}$ Co MO showed as expected a normal twist expression of the three cranial-crest segments: mandibular (m), hyoid (h) and branchial (b) (Fig. 20A; Fig. 21A, B). By injecting 20 ng Chd7 MO to knockdown Chd7 almost $80 \%$ of the analysed embryos of the neurula and tailbud stage showed severe migration and induction defects of NCCs highlighted by red arrowheads (Fig. 20B; Fig. 21C, D). About half of the embryos at the neurula stage revealed moderate migrating defects of NCCs (yellow arrowheads) after Sema3a (Fig. 20C) and Sema3d (Fig. 20D) downregulation. Furthermore, in about $5 \%$ of the analysed 
embryos severe migration defects were detected after a Sema3a knockdown. If Sema3d is downregulated, $10 \%$ of the embryos revealed severe defects of migrating NCCs. About $20 \%$ of the embryos at the tailbud stage showed moderate migration defects after Sema3a was downregulated (Fig. 21E, F) and in almost $40 \%$ of the embryos moderate defects of migrating NCCs were observed after downregulation of Sema3d indicated by yellow arrowheads (Fig. 21G, H). A rescue of the Chd7 MO phenotype (twist expression) was successfully performed using the same human CHD7 RNA as a rescue construct as described before on page 109 (results not shown). This data confirms the results of the rescue experiment with Xenopus laevis embryos performed by Bajpai et al. (2010). In conclusion, a loss of function of Chd7 severely affects the NCC induction and migration in the majority of analysed embryos while a loss of function of either Sema3a or Sema3d led in about half of the embryos to moderate NCC migration defects.
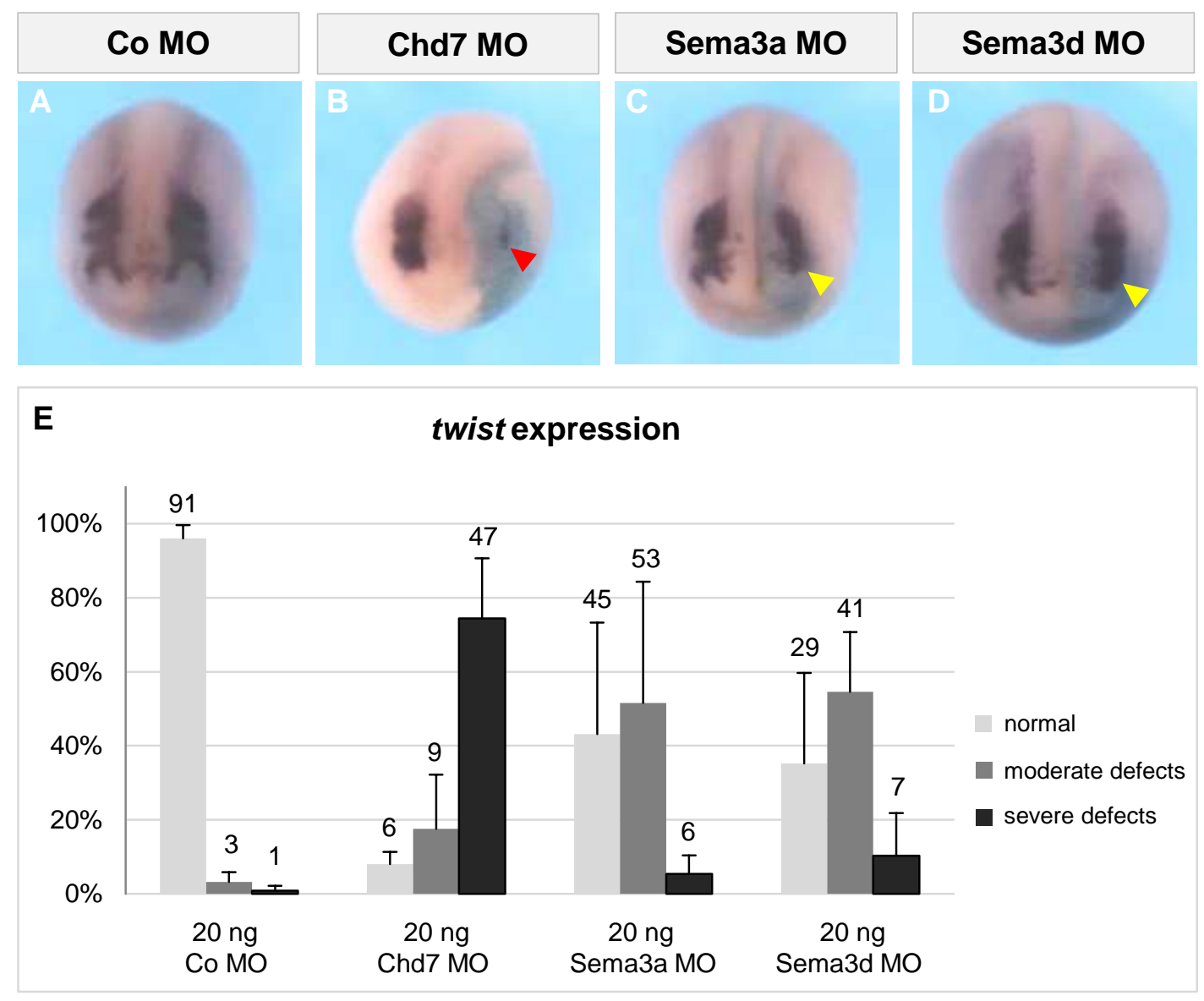

Figure 20: Twist expression analysis after knockdown of Chd7, Sema3a or Sema3d in Xenopus laevis (neurula stage 21).

Embryos were injected with $20 \mathrm{ng}$ Co MO (A), $20 \mathrm{ng}$ Chd7 MO (B), $20 \mathrm{ng}$ Sema3a MO (C) or $20 \mathrm{ng}$ Sema3d MO (D) in one blastomere of a two-cell stage. LacZ RNA was co-injected as a lineage tracer. 


\section{Results}

At the neurula stage 21 a whole mount in situ hybridisation was performed to analyse the expression of the NCC marker twist (purple staining). The injected side is indicated by the blue lacZ staining (B, D, F, H). More than $90 \%$ of the embryos injected with $20 \mathrm{ng}$ Co MO revealed a normal twist expression (A). After injecting $20 \mathrm{ng}$ Chd7 MO more than $70 \%$ showed severe defects in form of extreme migration or induction defects of NCCs (red arrowhead) (B). A knockdown of Sema3a and Sema3d led in about $50 \%$ of the embryos to moderate migration defects of NCCs (yellow arrowheads) (C, D). The diagram represents the percentage of embryos after injection of MO showing normal twist expression indicating normal NCC migration (light grey), moderate (grey) or severe migration defects (black) of NCCs (E). (I) The data include three independent experiments. Standard error of the means is given. Total numbers of animals dedicated to each category are shown on top of each column.

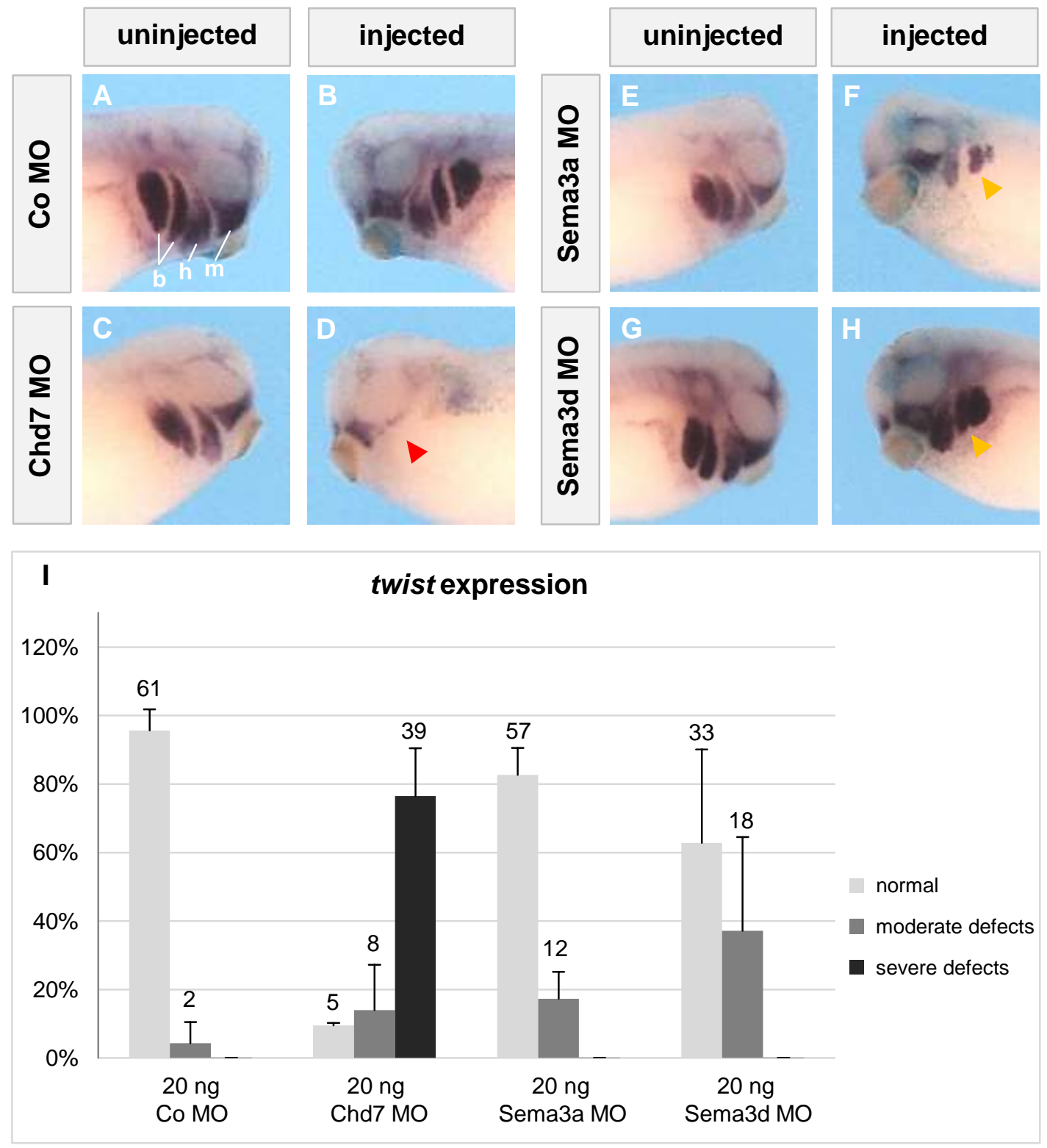

Figure 21: Effect of Chd7, Sema3a or Sema3d knockdown on NCCs (twist expression) in Xenopus laevis (tailbud stage 26). 
One blastomere of a two-cell stage was injected with a MO in combination with lacZ RNA. The injected side of the embryo is recognisable by the blue lac $Z$ staining $(\mathbf{B}, \mathbf{D}, \mathbf{F}, \mathbf{H})$. Control embryos were injected with $20 \mathrm{ng}$ of Co MO (A, B). For the knockdown $20 \mathrm{ng}$ Chd7 MO (C, D), $20 \mathrm{ng}$ Sema3a MO (E, F) or $20 \mathrm{ng} \mathrm{Sema3d} \mathrm{MO} \mathrm{were} \mathrm{injected}(\mathbf{G}, \mathbf{H})$. At the tailbud stage 26 a whole mount in situ hybridisation was performed to analyse the expression pattern of the NCC marker twist. Most embryos (> $90 \%$ ) injected with $20 \mathrm{ng}$ Co MO showed a normal twist expression pattern of the three cranial-crest segments: mandibular (m), hyoid (h) and branchial (b) (A, B). After injecting $20 \mathrm{ng}$ Chd7 MO to knockdown Chd7, in almost $80 \%$ of the analysed embryos severe defects were observed like massive migration and induction defects (red arrowhead) of NCCs (D). About $20 \%$ of the embryos injected with $20 \mathrm{ng}$ Sema3a MO (F) and $40 \%$ of those injected with $20 \mathrm{ng}$ Sema3d MO (H) showed moderate migration defects of NCCs (yellow arrowhead). In the diagram the results of two independent experiments are shown (I). The columns represent the percentage of embryos showing a normal NCC migration indicated by a normal twist expression (light grey), moderate migration defects (grey) or severe migration or induction defects of NCCs (black). The total number of embryos obtained in each category is shown on top of the columns. Standard error of the means is given.

\subsection{Double knockdown of Chd7 and Sema3a or Sema3d has no clear synergistic effect on twist expression in Xenopus laevis}

To determine whether the Chd7 MO phenotype on twist expression can be increased, double knockdown experiments on Xenopus laevis embryos were performed. Chd7 was downregulated in combination with either Sema3a or Sema3d. Embryos were injected in one blastomere of a two-cell stage. Twist expression was analysed by WMISH at the neurula stage 20 (Fig. 22A) and the tailbud stage 27 (Fig. 22B), respectively. Uninjected embryos as well as embryos injected with $10 \mathrm{ng}$ Co MO served as controls showing a normal twist expression. Chd7 alone was downregulated by injecting $10 \mathrm{ng}$ Chd7 MO. $35 \%$ of the embryos at the neurula stage revealed moderate migration defects and further $35 \%$ showed severe migration defects of NCCs (Fig. 22A). At the tailbud stage about $60 \%$ of the analysed embryos revealed severe alteration in twist expression after Chd7 was downregulated (10 ng Chd7 MO) (Fig. 22B). Embryos co-injected with 5 ng Chd7 MO and 5 ng Co MO were used as a reference control. Compared to the reference control, embryos (neurula stage) co-injected with $5 \mathrm{ng}$ Chd7 $\mathrm{MO}$ and $5 \mathrm{ng}$ Sema3a MO showed a slightly increase in moderate migration defects in NCCs. While no severe migration defects were detected in the reference control, $4 \%$ of the embryos revealed severe migration defects after the Chd7/Sema3a knockdown (Fig. 22A). In $28 \%$ of the 
Results

reference control embryos (tailbud stage) moderate defects in migrating NCCs were observed, while $48 \%$ were detected after a double knockdown of Chd7 and Sema3a (Fig. 22B). The combination of $5 \mathrm{ng}$ Chd7 MO and $5 \mathrm{ng}$ Sema3d MO led in $75 \%$ of the embryos of the neurula stage to moderate migration defects while in the reference control only $63 \%$ of the embryos were detected with moderate migration defects of NCCs (Fig. 22A). In tailbud stage embryos the percentage of embryos showing moderate defects of migrating NCCs was increased (39\%) compared to the reference control $(28 \%)$. Furthermore, $5 \%$ showed severe migration and induction defects whereby none of the reference control embryos were detected with this phenotype (Fig. 22B). 


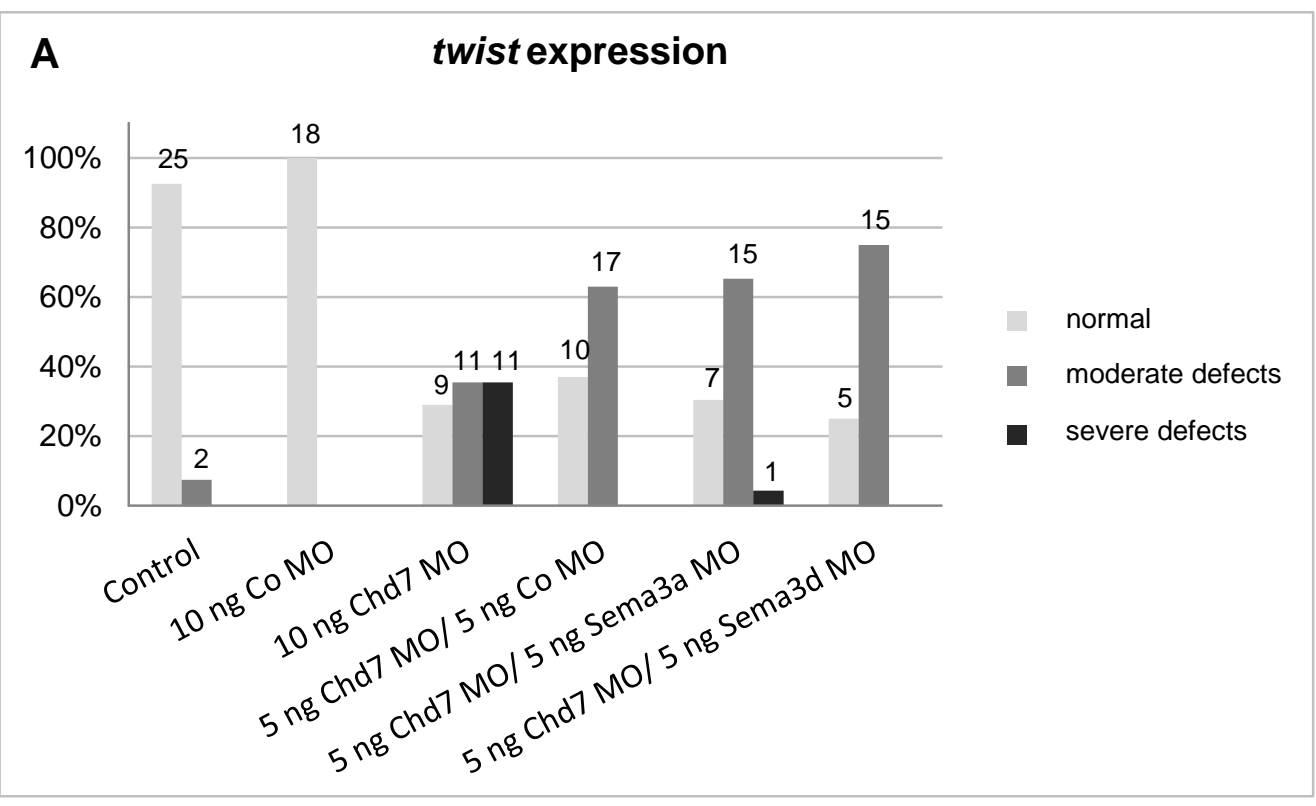

\section{B} twist expression

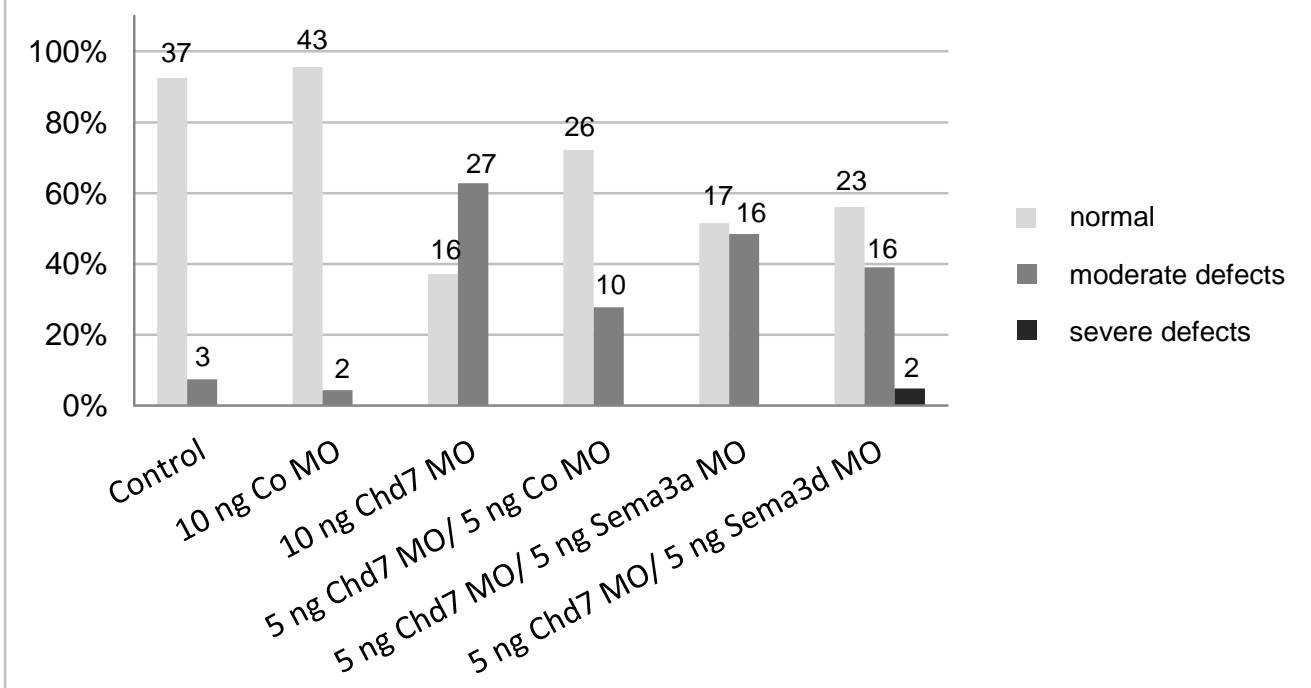

Figure 22: Effect on twist expression after Chd7 knockdown, Chd7/Sema3a and Chd7/Sema3d double knockdown in Xenopus laevis.

Embryos from one batch were either not injected or injected with $10 \mathrm{ng}$ of Co MO, $10 \mathrm{ng}$ Chd7 MO or co-injected with $5 \mathrm{ng}$ Co MO and 5 ng Chd7 MO, 5 ng Chd7 MO and 5 ng Sema3a MO or 5 ng Chd7 MO and $5 \mathrm{ng} \mathrm{Sema3d} \mathrm{MO.} \mathrm{At} \mathrm{neurula} \mathrm{stage} 20$ a whole mount in situ hybridisation was performed to analyse the expression of the NCC marker twist (A). The diagram summarises the results of one experiment. The columns represent the percentage of the embryos showing a normal expression of twist (normal NCC migration) (light grey), moderate migration defects (grey) or severe migration and induction defects of NCCs (black). Numbers on top of each column indicate the number of embryos found in the different categories. Nearly all control embryos (uninjected and injected with 10 ng Co MO) showed a normal twist expression. After injecting $10 \mathrm{ng}$ Chd7 MO, $35 \%$ of the embryos showed severe migration and induction defects of NCCs and $35 \%$ had moderate migration defects. In about $60 \%$ of embryos co-injected with $5 \mathrm{ng}$ Co MO and $5 \mathrm{ng}$ Chd7 MO moderate migration defects of 
NCCs were observed but no severe defects. After co-injection of 5 ng Chd7 MO and 5 ng Sema3a MO, more than $60 \%$ of the embryos showed moderate migration defects. In $4 \%$ severe migration defects were detected. A co-injection of $5 \mathrm{ng}$ Chd7 MO and $5 \mathrm{ng}$ Sema3d MO led in almost $80 \%$ of the embryos to moderate migration defects of NCCs. Another whole mount in situ hybridisation (one biological replicate) was performed at the tailbud stage 27 (B). More than $95 \%$ of the uninjected embryos and embryos injected with $10 \mathrm{ng}$ Co MO showed a normal twist expression pattern. After an injection of $5 \mathrm{ng}$ Co $\mathrm{MO}$ and $5 \mathrm{ng}$ Chd $7 \mathrm{MO}$, about $30 \%$ of the embryos had moderate migration defects. An injection of $10 \mathrm{ng}$ Chd7 MO led in approximately $60 \%$ of the embryos to moderate migration defects of NCCs. Half of the embryos showed moderate defects in migrating NCCs if $5 \mathrm{ng}$ Chd7 MO and $5 \mathrm{ng}$ Sema3a MO were injected. After co-injection of $5 \mathrm{ng}$ Chd7 MO and $5 \mathrm{ng}$ Sema3d MO, about $40 \%$ of the embryos revealed moderate migration defects and $5 \%$ showed severe migration defects of NCCs.

To analyse whether the increase of defects seen of NCC migration and induction after the double knockdown (Chd7 and Sema3a or Sema3d) can be intensified, the MO concentration were doubled to $10 \mathrm{ng}$. The expression pattern of twist was analysed in four independent experiments in neurula stages 20/21 (Fig. 23) and the tailbud stages 27, respectively (Fig. 23B). About $80 \%$ of the embryos showed severe migration and induction defects of NCCs after a downregulation of Chd7 by injecting $20 \mathrm{ng}$ Chd7 MO. Embryos, co-injected with $10 \mathrm{ng}$ Chd7 MO and $10 \mathrm{ng}$ Co MO were used as a reference control. In comparison of embryos of the reference control to embryos injected with either $10 \mathrm{ng}$ Sema3a MO or Sema3d MO instead of the Co MO, the percentage of embryos showing defects of NCC migration was increased.

After a Chd7/Sema3a double knockdown, more embryos (neurula stage) revealed severe migration and induction defects $(68 \%)$ as the reference control $(56 \%)$. The percentage of embryos with moderate defects of NCC migration was also increased (23\%) in comparison to the reference control (12\%). No changes in the percentage of embryos with defects of NCC migration were observed in tailbud stages.

Comparing the embryos after a double knockdown of Chd7/Sema3d to the reference control, it was observed that the percentage of embryos showing severe migration and induction defects was increased from $56 \%$ to $73 \%$. The percentage of embryos with moderate migration defects were slightly increased as well. In the tailbud stages a higher percentage of severe migration and induction defects was detected after Chd7/Sema3d double knockdown (62\%) compared to the reference control (48\%). 
Furthermore, it was observed in the neurula and tailbud stages that a double knockdown mediated by an injection of $10 \mathrm{ng}$ Chd7 MO + $10 \mathrm{ng}$ Sema3a MO or Sema3d MO did not reach the percentage of embryos showing defects in the migration of NCCs detected after single knockdown of Chd7 by injecting $20 \mathrm{ng}$ Chd7 MO. Although, a higher percentage of embryos showed defects of NCC migration and induction after downregulation of Chd7 in combination with Sema3a or Sema3d the effect is to less to conclude a clear synergistic effect. All experiments with the model organism Xenopus laevis were performed within the collaboration with Prof. Dr. Annette Borchers and Dr. Peter Wehner in the Department of Developmental Biochemistry, Goettingen. Handling of Xenopus laevis, including fertilisation, injecting embryos and caring until embryos reached the desired developmental stage has been undertaken by Dr. Peter Wehner. 

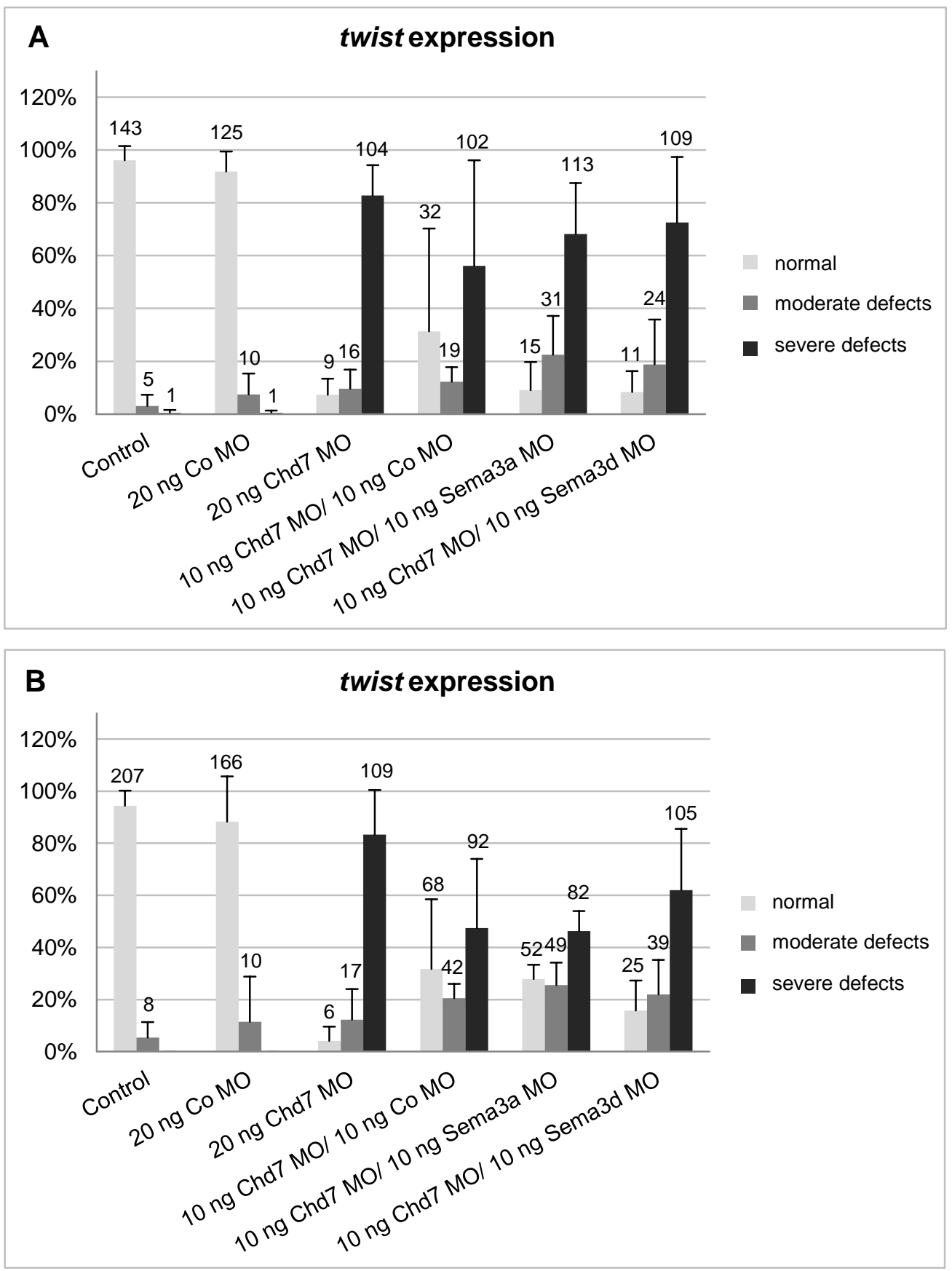

Figure 23: Effect on the NCC marker twist after Chd7 knockdown, Chd7/Sema3a and Chd7/Sema3d double knockdown in Xenopus laevis.

The graphs summarise four independent experiments where knockdowns were performed and the expression pattern of twist was analysed at the neurula stage 20/21 (A) and the tailbud stage 27 (B). The columns represent the percentage of the embryos showing a normal expression of twist indicating normal NCC migration (light grey), moderate migration defects (grey) or severe migration and induction defects of NCCs (black). Numbers on top of each column indicate the number of embryos obtained in the different categories. Standard error of the means is shown. At a two-cell stage embryos were injected in one blastomere with $20 \mathrm{ng}$ Co MO, $10 \mathrm{ng}$ Co MO and $10 \mathrm{ng}$ Chd7 MO, 20 ng Chd7 MO, $10 \mathrm{ng}$ Chd7 MO and $10 \mathrm{ng}$ Sema3a MO or $10 \mathrm{ng}$ Chd7 MO and $10 \mathrm{ng}$ Sema3d MO. The twist expression pattern remained unchanged in more than $90 \%$ of the uninjected embryos and after 


\section{Results}

injection of $20 \mathrm{ng}$ Co MO. An injection of $10 \mathrm{ng}$ Co MO and $10 \mathrm{ng}$ Chd7 MO led in more than $50 \%$ of the embryos to severe defects of migrating NCCs. In more than $80 \%$ of the embryos injected with 20 ng Chd7 MO severe migration and induction defects were observed. After double knockdown of Chd7 and Sema3a, about $70 \%$ of the embryos showed severe migration and induction defects of NCCs and more than $20 \%$ were observed with moderate migration defects. An injection of $10 \mathrm{ng}$ Chd7 MO and $10 \mathrm{ng}$ Sema3d MO led in more than $70 \%$ of the embryos to severe migration and induction defects and about $20 \%$ showed moderate defects of migrating NCCs. The whole mount in situ hybridisation performed at the tailbud stage revealed for the majority of uninjected and the injected embryos with $20 \mathrm{ng}$ Co MO a normal expression pattern of twist. More than $40 \%$ of embryos co-injected with $10 \mathrm{ng}$ Co MO and $10 \mathrm{ng}$ Chd7 MO showed severe defects of migrating NCCs and about $20 \%$ were detected with moderate migration defects. After downregulation of Chd7 by injecting $20 \mathrm{ng}$ Chd7 MO, more than $80 \%$ of the analysed embryos showed severe migration and induction defects of NCCs. More than $40 \%$ of the embryos were detected with severe migration and induction defects after a double knockdown of Chd7 and Sema3a by injecting $10 \mathrm{ng}$ of each MO and more than $20 \%$ showed moderate migration defects. A co-injection of $10 \mathrm{ng}$ Chd7 MO and $10 \mathrm{ng}$ Sema3d MO led in about $60 \%$ of the embryos to severe migration and induction defects and more than $20 \%$ revealed moderate defects of migrating NCCs. 


\section{Discussion}

\section{Discussion}

\subsection{Short summary}

It was shown in two independent experiments performed with HeLa cells that CHD7 interacts with WDR5, ASH2L and RBBP5 (WAR complex). By Co-IP experiments the interaction of CHD7 with members of the WAR complex was shown in two ways: by precipitation of endogenous CHD7 and detection of overexpressed ASH2L and RBBP5 (Fig. 5A) as well as by precipitation of endogenous WDR5 and overexpressed ASH2L and RBBP5 and detection of endogenous CHD7, respectively (Fig. 5C) (Schulz et al., 2014a). With the Duolink PLA method these interactions were confirmed and at the same time the localisation of the interactions was found to be in the nuclei of HeLa cells (Fig. 7) (Schulz et al., 2014a). Furthermore, Y2H experiments revealed no direct interaction of the analysed CHD7 fragment (amino acids 1591-2181) with full-length constructs of WDR5, ASH2L and RBBP5 (Fig. 8) (Schulz et al., 2014a).

The genome-wide microarray analysis performed with embryos of the Whirligig mouse line which carry a nonsense mutation in the $C h d 7$ gene, revealed 98 genes showing a differential expression (compare appendix, Tab. 39) (Schulz et al., 2014b). Interestingly, a lot of these genes are involved in the process of axon and NCC guidance, such as members of the semaphorin family SEMA3A, SEMA3C and SEMA3D (Fig. 10) (Schulz et al., 2014b).

Knockdown experiments of Chd7 carried out with embryos of the African clawed frog Xenopus laevis resulted in severe reduction or complete absence of sema3a expression (Fig. 16; Fig. 17) (Schulz et al., 2014b). Furthermore, the negative effect of a Chd7 downregulation on NCCs formation (twist expression) observed by Bajpai et al. (2010) was confirmed. After Chd7 downregulation, severe migration and induction defects of NCCs were determined (Fig. 20; Fig. 21). Downregulation of either Sema3a or Sema3d in Xenopus laevis embryos resulted in moderate migration defects (Fig. 20; Fig. 21). A double knockdown of Chd7 in combination with either Sema3a or Sema3d led to a higher number of Xenopus laevis embryos with more severe defects of migrating NCCs (Fig. 22; Fig. 23). 


\section{Discussion}

\subsection{CHARGE syndrome shows phenotypical overlap with other syndromes}

CHARGE syndrome is a highly heterogeneous disorder and shows a phenotypical overlap with other syndromes (Sanlaville and Verloes, 2007; Jongmans et al., 2009; Bergman et al., 2011). An example is the Kabuki syndrome (OMIM 147920) which is a rare developmental disorder characterised by a typical facial gestalt, short stature, skeletal abnormalities, cardiac malformations, immunological defects and intellectual disabilities (Ng et al., 2010; Li et al., 2011; Bogershausen and Wollnik, 2013). Heterozygous mutations in the KMT2D (MLL2) gene are described as the major genetic cause of Kabuki syndrome ( $\mathrm{Ng}$ et al., 2010). KMT2D is a methyltransferase responsible for histone 3 lysine 4 (H3K4) di- and trimethylation (Song JJ, 2008).

Our working group described a patient who was initially diagnosed as having CHARGE syndrome because he fulfilled the diagnostic criteria for CHARGE provided by Pagon et al. (1981) (five of six features) and Blake et al. (1998) (three of four major and three of seven minor signs). According to the CHARGE syndrome criteria from Verloes (2005), the patient fulfilled the criteria for having an atypical CHARGE syndrome (one of three major and three of five minor signs). To confirm the clinical diagnosis, a CHD7 mutation analysis was performed but no mutation was found. Further clinical examinations of the patient revealed emerging facial features, such as long palpebral fissures, long dense eyelashes and arched eye-brows, leading to the clinical diagnosis of Kabuki syndrome. A KMT2D mutation analysis revealed in exon 22 a heterozygous nonsense mutation confirming the clinical diagnosis of Kabuki syndrome (Schulz et al., 2014a).

\subsection{CHD7 is associated with the WAR complex}

$K M T 2 D$, the gene mutated in patients with Kabuki syndrome, belongs to the SET1 family of enzymes which are commonly associated with multi-subunit complexes to be able to activate the methyltransferase (Miller et al., 2001; Yokoyama et al., 2004; Wysocka et al., 2005; Steward et al., 2006; Ernst and Vakoc, 2012). In Drosophila the homologous gene of KMT2D is trx (trithorax) of the trithorax group which maintains the homeotic gene expression (Ringrose and Paro, 2004). The counterpart 
of trithorax group proteins are polycomb group proteins which are most commonly linked to the repression of homeotic genes (Ringrose and Paro, 2004).

One of the "core" complexes of KMT2D consists of WDR5, ASH2L and RBBP5 (WAR complex) (Steward et al., 2006; Song JJ, 2008; Yates et al., 2010). The ATPdependent chromatin remodelling enzyme CHD8 interacts with the components of the WAR complex in the absence of MLL (Steward et al., 2006; Thompson et al., 2008; Thompson BA, 2008; Yates et al., 2010). Yates et al. (2010) suggested a role of CHD8 in the regulation of gene transcription at the HOXA locus. They demonstrated a negative regulation of HOXA2 gene expression by CHD8 (Yates et al., 2010). Furthermore, it was shown by our working group that CHD7 interacts directly and indirectly with CHD8 (Batsukh et al., 2010), both proteins belong to the same subgroup of the CHD family (Flaus et al., 2006).

Co-IP experiments performed with HeLa cells demonstrated an interaction of CHD7 with WDR5, ASH2L and RBBP5 (Fig. 5) (Schulz et al., 2014a). Luisa Freese (PhD student of our group) completed successfully the Co-IP experiment using the pCMVHA-WDR5 construct, which was generated during writing my thesis. Full-length WDR5 was overexpressed in HeLa cells, endogenous CHD7 was precipitated and the overexpressed WDR5 was detected using an HA antibody (data not shown).

The interaction between CHD7 and members of the WAR complex was confirmed by the Duolink PLA method (Fig. 7) (Schulz et al., 2014a). Further, it was demonstrated that the interaction takes place in the nuclei of HeLa cells (Fig. 7) (Schulz et al., 2014a) where eukaryotic gene transcription takes place (Georgiev, 1972). Factors involved in the regulation of gene transcription such as the MLL2WAR methyltransferase complex are localised in the nucleus (MIPS et al., 2008) as well as the CHD family members of ATP-dependent chromatin remodelling enzymes CHD7 and CHD8 (Marfella and Imbalzano, 2007). In fact, it was shown by ICC that CHD7, CHD8 and the members of the WAR complex are specifically expressed in the nuclei of HeLa cells (Fig. 6).

Direct $\mathrm{Y} 2 \mathrm{H}$ experiments revealed no direct interaction for CHD7 and the WAR complex members (Fig. 8) (Schulz et al., 2014a). It is possible that the interaction region is located outside of the analysed CHD7 fragment (amino acids 1,591-2,181). Therefore, four overlapping fragments were generated spanning the whole CHD7 protein without interrupting any known functional domains (Fig. 9) (Schulz et al., 2014a). During writing this thesis, PhD student Luisa Freese performed direct Y2H 
experiments with the four CHD7 fragments. CHD7-1pGBKT7 showed an auto activation of the yeast strain reporter genes and the other three CHD7 fragments revealed no direct interaction with the WAR complex members (Schulz et al., 2014a).

As shown by Yates et al. (2010) CHD8 interacts directly with WDR5, ASH2L and RBBP5. The direct $\mathrm{Y} 2 \mathrm{H}$ experiments confirmed these interactions and narrowed down the responsible interaction side of CHD8 to the amino acids 1,789-2,302 (Fig. 8) (Schulz et al., 2014a). Furthermore, the recently shown direct interaction of the CHD7 fragment and the CHD8 fragment was observed (Fig. 8) (Batsukh et al., 2010; Schulz et al., 2014a). These data indicate that CHD7 is associated to the WAR complex most likely via the direct interaction with CHD8 (Schulz et al., 2014a). The association of CHD7 and KMT2D with the WAR complex connects these two genes to the same process of remodelling and modifying chromatin (Schulz et al., 2014a). Probably, CHD7 and KMT2D regulate the transcription of a subset of common genes (Schulz et al., 2014a). Therefore, it is likely that a mutation in either CHD7 or KMT2D causes misregulation of common target genes, which could explain the phenotypic overlap of CHARGE syndrome and Kabuki syndrome (Schulz et al., 2014a).

\subsection{CHD7 regulates genes required for proper NCC development}

CHD7 is included within different complexes regulating time and tissue specific gene expression (Layman et al., 2010). Already in 1985, several years before mutations in CHD7 were discovered to cause CHARGE syndrome, Siebert et al. (1985) postulated that a disturbance in NCC development is the pathogenic mechanism behind CHARGE syndrome. Recent studies demonstrated the involvement of Chd7 in NCC development (Bajpai et al., 2010). Bajpai et al. (2010) showed that Chd7 activates components, such as Slug, Sox9 and Twist which are critical for the formation of migratory NCCs. Furthermore, Bajpai et al. (2010) found that Chd7 binds to the PBAF complex and assumed that Chd7 cooperates with the PBAF complex to regulate gene expression during NCC formation in Xenopus laevis. In the mouse CHD7 is required in the pharyngeal ectoderm for normal pharyngeal arch artery development and it is likely that NCCs are the targets of 
CHD7 based on alterations in NCC migration observed in Chd7 heterozygous mouse embryos (Randall et al., 2009).

To analyse which genes are regulated by CHD7, a genome-wide microarray analysis was performed with embryos of the Whirligig mouse line $\left(C h d 7^{+/+}\right.$, Chd $7^{\text {Whil+ }}$, $C h d 7^{W h i / W h i}$ ) at E9.5, a developmental stage during NCC migration (Schulz et al., 2014b). By using whole mouse embryos one important advantage is to identify genes involved in NCC development in different tissues although possible cancellation effects cannot be excluded (Schulz et al., 2014b). Differences in gene expression between females and males are present in several species, affecting not only sex chromosome linked genes, but also autosomal genes (Vawter et al., 2004; Yang et al., 2006; Zhang et al., 2009). To avoid gender specific effects on gene expression, the genome-wide microarray analysis was performed only with female mouse embryos. To confirm the data of the genome-wide microarray analysis by RT-qPCR, six genes were chosen which were identified to be differentially effected by CHD7. The expression of Sema3a, Sema3d and Epha3 was found to be downregulated while the expression of Sox 10 and Gfra2 was increased and the Trp53bp2 expression was unaffected (appendix, Tab. 39) (Schulz et al., 2014b). The RNA of female mouse embryos which was also used for the microarray analysis was used for RT-qPCR. A similar expression of the selected genes was observed confirming the microarray data (Fig. 11) (Schulz et al., 2014b). Additionally, these genes were analysed by another RT-qPCR performed on male embryos. A gender specific affect for the analysed genes Sema3a, Sema3d, Epha3, Sox10, Gfra2 and Trp53bp2 was excluded (Fig. 12) (Schulz et al., 2014b). Since embryos from different breedings were obtained and used for the RT-qPCR experiments, slight differences in the developmental stage might occur, explaining the differences in expression of the same gene between the biological replicates.

The microarray data revealed 98 differentially expressed genes (appendix, Tab. 39); many of them are involved in NCC migration and NCC and axons guidance as well as in interaction between NCCs and other tissues (Schulz et al., 2014b). For instance, several cadherins were identified which play a role in cell adhesion (Takeichi, 1995). Changes in cell adhesion are important during EMT which enables NCC migration (Kuriyama and Mayor, 2008). Transcription factors such as Sox10 and Foxd3 were found to be differentially expressed (Schulz et al., 2014b). They are involved in early migration processes of NCCs (Kirby and Hutson, 2010). Furthermore, Sox10 seems 


\section{Discussion}

to be important for the specification of NCCs (Gammill and Bronner-Fraser, 2003). In addition, secreted signalling molecules such as semaphorins, ephrins and slits were identified which act through protein kinase receptors to guide migrating NCCs (Perris, 1997; Gammill and Bronner-Fraser, 2003; Yazdani and Terman, 2006; Kuriyama and Mayor, 2008; Kirby and Hutson, 2010). Guidance molecules such as semaphorins and slits are expressed, for instance, in the surrounding epithelium of NCC localisation, guiding NCCs along specific pathways to their destination (Yazdani and Terman, 2006; Kuriyama and Mayor, 2008). Randall et al. (2009) observed that a rescue of CHD7 in murine NCCs alone did not rescue the phenotype of pharyngeal arch artery defects in CHD7 deficient mice whereas a rescue of CHD7 in NCCs and the pharyngeal ectoderm led to full rescue of pharyngeal arch artery alterations. Randall et al. (2009) assumed ephrins, Pdgfs (platelet-derived growth factors), semaphorins, slits and Vegfs (vascular endothelial growth factors) as downstream candidate genes of CHD7 involved in ectoderm-NCC interaction. The data from the microarray analysis (compare 3.4) revealed among others members of all signalling families postulated by Randall et al. (2009): Epha3, Epha5, Epha7 (ephrins), Pdgfc (Pdgfs), Sema3a, Sema3c, Sema3d (semaphorins), Slitrk6, Slitrk1 (slits) and Vegfc (Vegfs). It is likely that CHD7 regulates genes which expression is important in NCCs and the surrounding tissue to ensure proper NCC development (specification, migration and differentiation). Furthermore, the data of the microarray analysis indicate that disturbance of NCC development plays a role in the pathogenesis of CHARGE syndrome. It seems that additional pathogenic mechanisms exist behind CHARGE syndrome. Possibly, till now untested theories, are a disruption of the interaction of mesoderm and NCCs (Van Meter and Weaver, 1996) or the disturbance of mesenchymal-epithelial interaction (Williams, 2005).

\subsection{Chd7 regulates sema3a expression and NCC induction and migration in Xenopus laevis}

Chd7 is highly conserved across different species, for instance, human, mouse, zebrafish and Xenopus laevis (Bosman et al., 2005; Bajpai et al., 2010). Disruption of Chd7 function in Xenopus laevis exposed defects comparable to symptoms seen in CHARGE syndrome (Bajpai et al., 2010). A downregulation of Chd7 in Xenopus laevis embryos has an influence on the expression of the NCC marker twist 
demonstrating alteration in NCC migration (Bajpai et al., 2010). Disruption of CHD7 function in mouse was found to result in a decreased expression of members of the semaphorin family, such as Sema3a and Sema3d (Schulz et al., 2014b).

To analyse whether there is a conserved regulatory mechanism across species, knockdown experiments on Xenopus laevis embryos were performed injecting MO in one blastomere of a two-cell stage. Analysis of sema3a expression after downregulation of Chd7 caused either decreased or complete depletion of sema3a expression (Fig. 16; Fig. 17) (Schulz et al., 2014b), exposing a conserved mechanism between mouse and Xenopus laevis.

\subsection{SEMA3A and SEMA3D seem to play a role in the pathogenesis of CHARGE syndrome}

To test whether disruption of Sema3a or Sema3d phenocopies the NCC defects observed after Chd7 loss of function, knockdown experiments of Chd7, Sema3a and Sema3d were performed with Xenopus laevis embryos and the expression pattern of the NCC marker twist was analysed by WMISH. While a Chd7 loss of function caused severe migration and induction defects of NCCs, knockdown of Sema3a or Sema3d revealed moderate migration defects of NCCs (Fig. 20; Fig. 21). The severe NCC alterations observed in Xenopus laevis after Chd7 downregulation are likely caused by the regulatory effect of Chd7 on several genes involved in NCC formation and migration (Schulz et al., 2014b). Nevertheless, the semaphorins Sema3a and Sema3d partially phenocopy the NCC defects observed during the Chd7 loss of function indicating a possible participation of these semaphorins in the pathogenesis of CHARGE syndrome.

Double knockdown of Chd7 and Sema3a or Sema3d was performed to analyse whether there is a synergistic effect. The percentage of embryos showing severe migration defects of NCCs was clearly increased but insufficient to determine a synergistic effect. In the mouse a significant decreased expression of different semaphorins were shown (appendix, Tab. 39) and in Xenopus laevis a Chd7 loss of function caused also a reduced sema3a expression (Fig. 16; Fig. 17) (Schulz et al., 2014b). A clear synergistic effect after the double knockdown of Chd7 and Sema3a or Chd7 and Sema3d was not observed (Fig. 22; Fig. 23). A possible explanation could be that the Chd7 knockdown itself has such a strong negative effect on the 
expression of sema3a and sema3d that an additional knockdown of either Sema3a or Sema3d cannot cause a synergistic effect.

The CHD7 expression pattern studied in different species (human, mouse, chick and others) correlates with the malformations observed in CHARGE syndrome (Bosman et al., 2005; Sanlaville et al., 2006; Aramaki et al., 2007; Hurd et al., 2007; Layman et al., 2009; Bergman et al., 2010; Kosaki, 2011; Janssen et al., 2012). CHD7 is expressed, for instance, in the brain, heart, inner ear, olfactory epithelium and ganglia (Bosman et al., 2005; Lalani et al., 2006; Sanlaville et al., 2006; Aramaki et al., 2007; Hurd et al., 2007; Layman et al., 2009; Bergman et al., 2010). SEMA3D expression has been observed in bones and cartilage, heart, endothelial cells, fibroblasts, epidermis, glia and neurons (Luo et al., 1995; Halloran et al., 1999; Cohen et al., 2003; Serini et al., 2003; Lallier, 2004; Jin et al., 2006). In paraffin sections of mouse embryos (E12.5) CHD7 and SEMA3D showed an overlapping expression pattern in the medulla oblongata, choroid plexus, cochlea, heart, cartilage primordium of body of vertebra and in different ganglia (Fig.19) indicating a possible role of SEMA3D in the pathogenesis of CHARGE syndrome, although further studies are needed to clarify this aspect.

\subsection{Semaphorins might act as modifier in CHARGE syndrome}

Semaphorins are conserved secreted and membrane-associated proteins which can be divided into eight classes according to domain architecture and phylogenetic tree analysis ("Unified nomenclature for the semaphorins/collapsins. Semaphorin Nomenclature Committee," 1999; Yazdani and Terman, 2006). Classes 3-7 are found only in vertebrates (Yazdani and Terman, 2006). Class 3 of semaphorins was identified to be required for NCC migration (Eickholt et al., 1999; Osborne et al., 2005; Yu and Moens, 2005).

The malformation disorder CHARGE syndrome was postulated to belong to the neurocristopathies (Siebert et al., 1985). Mutations in the CHD7 gene were identified as the genetic cause in about two-thirds of CHARGE syndrome patients (Vissers et al., 2004; Sanlaville and Verloes, 2007). In 5-10 \% of typical CHARGE patients and in 40-60\% of patients suspected of having CHARGE syndrome the cause remains unknown (Janssen et al., 2012). Several candidate genes, such as PAX2 (paired box 2) and PITX2 (paired-like homeodomain 2) were analysed but no mutations were 


\section{Discussion}

identified (Tellier et al., 2000; Martin et al., 2002). In a CHARGE syndrome patient originally described by Martin et al. (2001) with a de novo balanced translocation involving chromosomes 2 and 7, Lalani et al. (2004) mapped the translocation breakpoints and identified SEMA3E within $200 \mathrm{~kb}$ of the breakpoint on 7q21.11. By screening additional patients with CHARGE syndrome for mutations in the SEMA3E gene a de novo mutation was found (S703L) in an unrelated patient (Lalani et al., 2004). The S703L mutation was not found in the parents (Lalani et al., 2004). CHD7 mutations were not detected in these two patients, but $C H D 7$ deletions could not be excluded (Lalani et al., 2006).

While the Sema3e expression was found to be unchanged in the genome-wide microarray analysis performed on Whirligig mouse embryos described in section 3.4, the expression of Sema3a, Sema3c and Sema3d were significantly reduced (Schulz et al., 2014b). Additional sequencing of the SEMA3C gene that is located $1.9 \mathrm{Mb}$ centromeric from the translocation breakpoint in 24 patients revealed no mutation (Lalani et al., 2004). The SEMA3D gene lies with SEMA3E on $7 \mathrm{q} 21.11$, so it is possible that its function is disturbed by the translocation (Database, 2014a, b).

Sema3a knockout mice show altered olfactory bulb innervation and hypogonadism caused by abnormal development of Gonadotropin-releasing hormone ( $\mathrm{GnRH})$ neurons exhibiting a phenotype similar to Kallmann syndrome (Schwarting et al., 2000; Cariboni et al., 2011). Kallmann syndrome is a developmental disorder associated with idiopathic hypogonadotropic hypogonadism and congenitally absent or impaired sense of smell (anosmia) (Kallmann et al., 1944). Both features are also found in patients with CHARGE syndrome (Chalouhi et al., 2005; Pinto et al., 2005; Asakura et al., 2008; Blustajn et al., 2008). Additional abnormalities may occur in patients with Kallmann syndrome, such as cleft lip and palate, dental agenesis, unilateral renal agenesis, abnormal eye movements and neurological alterations (Santen and Alvin Paulsen, 1972; Wegenke et al., 1975; Lieblich et al., 1982; Schwankhaus et al., 1989; Hardelin et al., 1993a; Hardelin et al., 1993b; Kirk et al., 1994; de Zegher et al., 1995; Molsted et al., 1997; Soderlund et al., 2002; Dode et al., 2003; Kim et al., 2008a). Among others, mutations in CHD7 were identified in some patients with Kallmann syndrome (Kim et al., 2008b; Jongmans et al., 2009). It was assumed that Kallmann syndrome represents the mild end of CHARGE syndrome (Kim et al., 2008b; Jongmans et al., 2009). 


\section{Discussion}

Young et al. (2012) reported a SEMA3A deletion in a family with Kallmann syndrome. A screen of 386 patients with Kallmann syndrome for mutations in SEMA3A revealed non-synonymous mutations in 24 patients (Hanchate et al., 2012). As described before, semaphorins seem to be involved in the pathogenesis of CHARGE syndrome. To analyse whether semaphorins contribute to the phenotype of CHARGE syndrome, 45 patients diagnosed with CHARGE but lacking a mutation in the $C H D 7$ gene were sequenced for the presence of mutations in the coding sequence of SEMA3A and SEMA3D.

Three non-synonymous mutations for SEMA3A in a heterozygous state, namely c.196 C>T (p.R66W), c.2002 A>G (p.I668V) and c.2062 A>G (p.T688A), were identified (Fig.14) (Schulz et al., 2014b). Interestingly, two of the identified missense mutations (p.R66W and p.T688A) were described in patients with Kallmann syndrome (Hanchate et al., 2012). Hanchate et al. (2012) exposed that the missense mutation p.R66W results in altered secretion of SEMA3A while p.T668A causes a reduced signalling activity of SEMA3A. Based on these findings a pathogenic effect in Kallmann syndrome was assumed (Hanchate et al., 2012). Further, it was postulated that a heterozygous mutation in SEMA3A alone is not sufficient to induce the phenotypic features of Kallmann syndrome, while in combination with mutations in other Kallmann syndrome causing genes it could contribute to it (Hanchate et al., 2012).

It was shown in this work that the non-synonymous mutation (p.R66W) identified in a CHARGE patient was inherited by the healthy father (Schulz et al., 2014b).

A mutation screen of the coding sequence of SEMA3D in 45 CHD7 negative CHARGE patients revealed no disease causing nucleotide alteration nor a small deletion or insertion. Three non-synonymous mutations were identified in a heterozygous state, namely c.193 T>C (p.S65P), c.1272 C>A (p.H424Q) and c.2101 $A>C$ (p.K701Q) (Fig. 15). The missense mutation p.His424Gln affects a conserved residue. While the common variant p.K701Q (allele frequency of 0.346 in the ESP cohort population) and the less common variant p.S65P (allele frequency of 0.017 in the ESP cohort population) are predicted to be benign (Polyphen and Polyphen2), the missense mutation p.H424Q with an allele frequency of 0.002 is predicted to be damaging (Polyphen, Polyphen2, Sift). Screening the parents of the patient carrying the p.H424Q variant revealed that the variation was inherited by the healthy father. These results indicate that heterogeneous mutations in SEMA3A or SEMA3D are not 
sufficient to cause CHARGE syndrome but it is possible that these mutations contribute to the pathogenesis of CHARGE syndrome as suggested for SEMA3A mutations for Kallmann syndrome (Hanchate et al., 2012). CHARGE syndrome is a highly heterogeneous malformation disorder showing inter- and intra-familial heterogeneity (Aramaki et al., 2006; Jongmans et al., 2006; Lalani et al., 2006). Mutations in other genes like SEMA3A and SEMA3D might act as modifiers leading to a more severe phenotype explaining the heterogeneous appearance of patients with CHARGE syndrome (Schulz et al., 2014b).

\subsection{Future perspective}

Since CHD7 is associated with different complexes to regulate gene expression in a developmental stage and tissue specific manner, it would help to analyse the composition of $\mathrm{CHD} 7$ protein complexes. One method to analyse the structure and composition of especially heterogenic protein complexes is by chemical crosslinking combined with mass spectrometry (XL-MS) (Herzog et al., 2012). Furthermore, arrangement of proteins within complexes and the interplay of protein complexes in a signal transduction pathway can be investigated.

CHARGE syndrome is a phenotypic variable malformation syndrome and in the present work it is assumed that SEMA3A and SEMA3D might have modifying functions contributing to a more severe phenotype if they are mutated. Therefore, CHARGE patients carrying a CHD7 mutation should be screened for additional mutations in SEMA3A and SEMA3D. Especially familial cases showing phenotypic variations would be of great value.

For the Sema3a and Sema3d knockdown experiments performed with Xenopus laevis embryos, rescue experiments have to be accomplished to prove that the observed phenotype is caused by the loss of Sema3a and Sema3d function.

The genome-wide microarray analysis revealed 98 differentially expressed genes. Several of these genes play a role in NCC development (specification, EMT, guidance of NCC migration and interaction of NCCs with other tissues). Functional analysis of other guidance factors, such as ephrins or slits can be performed which are also involved in axon guidance during neurogenesis (Kirby and Hutson, 2010). This is another important direction since neurologic disturbances exist in CHARGE 
syndrome, for instance, cranial nerve palsy, arhinencephaly or abnormal olfactory bulbs and anosmia (Chalouhi et al., 2005; Sanlaville et al., 2005).

Further expression analysis by WMISH or immunohistochemistry in the mouse is recommendable. It would be interesting to analyse the expression pattern of SEMA3A, SEMA3D as well as CHD7 in embryos (wild-type $\left(C h d 7^{+/+}\right)$, heterozygous $\left(C h d 7^{W h i /+}\right)$ and homozygous $\left(C h d 7^{\text {Whi/Whi }}\right)$ ) of the Whirligig mouse line. Furthermore, NCC migration and development should be checked in these mice. Disturbances in NCC migration can be visualised by staining the expression pattern of the NCC marker Twist and the correct NCC differentiation can be checked, for instance, by observing the formation of dorsal root ganglia. Another possibility to analyse the process of NCC migration in vitro is to plate NCCs on a fibronectin layer (Rovasio et al., 1983). Fibronectin is one component of the extracellular matrix which migrating NCCs encounter (Tosney, 1978; Lofberg et al., 1980; D. Newgreen and Thiery, 1980; Duband and Thiery, 1982; D. F. Newgreen et al., 1982; Spieth and Keller, 1984; Brauer et al., 1985; Duband et al., 1986; Krotoski et al., 1986). It would be interesting to test the guidance potential of SEMA3A and SEMA3D on NCCs from wild-type $\left(C h d 7^{+/+}\right)$, heterozygous $\left(C h d 7^{W h i /+}\right)$ and homozygous $\left(C h d 7^{\text {Whi/Whi }}\right)$ mice by explanting NCCs on fibronectin containing matrices with alternating stripes of the semaphorin. Furthermore, the described non-synonymous mutations identified in the SEMA3A and SEMA3D screen in 45 CHD7 negative CHARGE patients could be induced by side-directed mutagenesis into a wild-type semaphorin plasmid to generate a semaphorin with this defect and to test whether these mutations cause alterations in the guidance of migrating NCCs. 


\section{References}

Abcam. (n.d.). Immunocytochemistry $\quad$ (ICC) protocol. http://docs.abcam.com/pdf/protocols/Immunocytchemistry-ICC-protocol.pdf (Effective: 02/08/2014).

AgilentTechnologies. (n.d.). PfuUltra High-Fidelity DNA Polymerase https://www.chem.agilent.com/Library/usermanuals/Public/600380.pdf (Effective: 01/08/2014).

Ambion. (2012). mMESSAGE mMACHINE® ${ }^{2}$ Kit. http://tools.lifetechnologies.com/content/sfs/manuals/cms_055516.pdf (Effective: 01/08/2014).

Antipenko, A., Himanen, J.P., van Leyen, K., Nardi-Dei, V., Lesniak, J., Barton, W.A., Rajashankar, K.R., Lu, M., Hoemme, C., Puschel, A.W., and Nikolov, D.B. (2003). Structure of the semaphorin-3A receptor binding module. Neuron 39, 589-598.

AppliedBiosystems. (n.d.). Allelic Discrimination Getting Started Guide http://tools.lifetechnologies.com/content/sfs/manuals/cms_042114.pdf (Effective: 01/08/2014).

Aramaki, M., Kimura, T., Udaka, T., Kosaki, R., Mitsuhashi, T., Okada, Y., Takahashi, T., and Kosaki, K. (2007). Embryonic expression profile of chicken CHD7, the ortholog of the causative gene for CHARGE syndrome. Birth defects research. Part A, Clinical and molecular teratology 79, 50-57.

Aramaki, M., Udaka, T., Kosaki, R., Makita, Y., Okamoto, N., Yoshihashi, H., Oki, H., Nanao, K., Moriyama, N., Oku, S., Hasegawa, T., Takahashi, T., Fukushima, Y., Kawame, H., and Kosaki, K. (2006). Phenotypic spectrum of CHARGE syndrome with CHD7 mutations. The Journal of pediatrics 148, 410-414.

Arber, W., and Linn, S. (1969). DNA modification and restriction. Annual review of biochemistry $38,467-500$.

Asakura, Y., Toyota, Y., Muroya, K., Kurosawa, K., Fujita, K., Aida, N., Kawame, H., Kosaki, K., and Adachi, M. (2008). Endocrine and radiological studies in patients with molecularly confirmed CHARGE syndrome. The Journal of clinical endocrinology and metabolism 93, 920-924.

Atkinson, M.R., Deutscher, M.P., Kornberg, A., Russell, A.F., and Moffatt, J.G. (1969). Enzymatic synthesis of deoxyribonucleic acid. XXXIV. Termination of chain growth by a 2',3'-dideoxyribonucleotide. Biochemistry 8, 4897-4904.

Bajpai, R., Chen, D.A., Rada-Iglesias, A., Zhang, J., Xiong, Y., Helms, J., Chang, C.P., Zhao, Y., Swigut, T., and Wysocka, J. (2010). CHD7 cooperates with PBAF to control multipotent neural crest formation. Nature 463, 958-962.

Batsukh, T., Pieper, L., Koszucka, A.M., von Velsen, N., Hoyer-Fender, S., Elbracht, M., Bergman, J.E., Hoefsloot, L.H., and Pauli, S. (2010). CHD8 interacts with CHD7, a protein which is mutated in CHARGE syndrome. Human molecular genetics 19, 2858-2866.

Batsukh, T., Schulz, Y., Wolf, S., Rabe, T.I., Oellerich, T., Urlaub, H., Schaefer, I.M., and Pauli, S. (2012). Identification and characterization of FAM124B as a novel component of a CHD7 and CHD8 containing complex. PloS one 7, e52640.

Bergman, J.E., Bosman, E.A., van Ravenswaaij-Arts, C.M., and Steel, K.P. (2010). Study of smell and reproductive organs in a mouse model for CHARGE syndrome. European journal of human genetics : EJHG 18, 171-177. 
Bergman, J.E., Janssen, N., Hoefsloot, L.H., Jongmans, M.C., Hofstra, R.M., and van Ravenswaaij-Arts, C.M. (2011). CHD7 mutations and CHARGE syndrome: the clinical implications of an expanding phenotype. Journal of medical genetics 48, 334-342.

BIO-RAD. (n.d.). Mini-Trans-Blot® Electrophoretic Transfer Cell http://www.bio$\mathrm{rad} . \mathrm{com} / \mathrm{webroot} / \mathrm{web} / \mathrm{pdf} / \mathrm{lsr} / \mathrm{literature/M1703930.pdf}$

(Effective: 01/08/2014).

Bioline. (n.d.). IMMOLASETM DNA Polymerase http://www.bioline.com/downloads/dl/file/id/872/immolase_dna_polymerase _manual.pdf (Effective: 31/07/2014).

Birnboim, H.C., and Doly, J. (1979). A rapid alkaline extraction procedure for screening recombinant plasmid DNA. Nucleic acids research 7, 1513-1523.

Blake, K.D., Davenport, S.L., Hall, B.D., Hefner, M.A., Pagon, R.A., Williams, M.S., Lin, A.E., and Graham, J.M., Jr. (1998). CHARGE association: an update and review for the primary pediatrician. Clin Pediatr (Phila) 37, 159173.

Blustajn, J., Kirsch, C.F., Panigrahy, A., and Netchine, I. (2008). Olfactory anomalies in CHARGE syndrome: imaging findings of a potential major diagnostic criterion. AJNR. American journal of neuroradiology 29, 12661269.

Bogershausen, N., and Wollnik, B. (2013). Unmasking Kabuki syndrome. Clinical genetics 83, 201-211.

Bosman, E.A., Penn, A.C., Ambrose, J.C., Kettleborough, R., Stemple, D.L., and Steel, K.P. (2005). Multiple mutations in mouse Chd7 provide models for CHARGE syndrome. Human molecular genetics 14, 3463-3476.

Bradford, M.M. (1976). A rapid and sensitive method for the quantitation of microgram quantities of protein utilizing the principle of protein-dye binding. Analytical biochemistry 72, 248-254.

Brauer, P.R., Bolender, D.L., and Markwald, R.R. (1985). The distribution and spatial organization of the extracellular matrix encountered by mesencephalic neural crest cells. The Anatomical record 211, 57-68.

Brown, C.B., Feiner, L., Lu, M.M., Li, J., Ma, X., Webber, A.L., Jia, L., Raper, J.A., and Epstein, J.A. (2001). PlexinA2 and semaphorin signaling during cardiac neural crest development. Development 128, 3071-3080.

Bustin, S.A., Benes, V., Garson, J.A., Hellemans, J., Huggett, J., Kubista, M., Mueller, R., Nolan, T., Pfaffl, M.W., Shipley, G.L., Vandesompele, J., and Wittwer, C.T. (2009). The MIQE guidelines: minimum information for publication of quantitative real-time PCR experiments. Clinical chemistry 55, 611-622.

Cariboni, A., Davidson, K., Rakic, S., Maggi, R., Parnavelas, J.G., and Ruhrberg, C. (2011). Defective gonadotropin-releasing hormone neuron migration in mice lacking SEMA3A signalling through NRP1 and NRP2: implications for the aetiology of hypogonadotropic hypogonadism. Human molecular genetics 20 , 336-344.

Chalouhi, C., Faulcon, P., Le Bihan, C., Hertz-Pannier, L., Bonfils, P., and Abadie, V. (2005). Olfactory evaluation in children: application to the CHARGE syndrome. Pediatrics 116, e81-88.

Clontech_Laboratories. (2008). pGBKT7 Vector Information. http://www.google.de/url?sa=t\&rct=j\&q=\&esrc=s\&source=web\&cd=1\&ved $=0 \mathrm{CCIQFjAA} \& u r l=\mathrm{http} \% 3 \mathrm{~A} \% 2 \mathrm{~F} \% 2 \mathrm{Fwww}$. clontech.com\%2Fxxclt_ibcGetA 
ttachment.jsp\%3FcItemId\%3D17639\&ei=kQbzU7HfG-

HIyAO70IG4Dg\&usg=AFQjCNEEUsnDoR_EVk2vIAjpZat5uqUj4Q\&bvm= bv.73231344,d.bGQ (Effective: 19/08/2014).

Clontech_Laboratories. (2009a). Matchmaker® Gold Yeast Two-Hybrid System User

Manual.

http://www.google.de/url?sa=t\&rct=j\&q=\&esrc=s\&source=web\&cd=3\&ved $=0 \mathrm{CDQQFjAC} \& u r l=\mathrm{http} \% 3 \mathrm{~A} \% 2 \mathrm{~F} \% 2 \mathrm{Fwww}$. clontech.com\%2Fxxclt_ibcGet Attachment.jsp\%3FcItemId\%3D17597\&ei=Dg_zU9swpL3KA9DxgeAJ\&usg =AFQjCNFpm8VCqNOXHa_3PgDVT0uDdNEj9g\&bvm=bv.73231344,d.b GQ (Effective: 19/08/2014).

Clontech_Laboratories. (2009b). Yeast Protocols Handbook http://www.google.de/url?sa=t\&rct=j\&q=\&esrc=s\&source=web\&cd=1\&ved $=0 \mathrm{CCcQFjAA \& url=http} \% 3 \mathrm{~A} \% 2 \mathrm{~F} \% 2 \mathrm{Fwww}$. clontech.com\%2Fxxclt_ibcGetA ttachment.jsp\%3FcItemId\%3D17602\&ei=dRXzUioIMfXyQPur4KQAQ\&usg=AFQjCNFWn9PqpRFCyAKX9dywFWOHBrx2Q\&bvm=bv.73231344,d.bGQ 02/08/2014).

Clontech_Laboratories. (2012). pGADT7 AD Vector Information. http://www.google.de/url?sa=t\&rct=j\&q=\&esrc=s\&source=web\&cd=1\&ved $=0 \mathrm{CCIQFjAA \& url=http} \% 3 \mathrm{~A} \% 2 \mathrm{~F} \% 2 \mathrm{Fwww} . c l o n t e c h . c o m \% 2 \mathrm{Fxxclt} \_$ibcGetA ttachment.jsp\%3FcItemId\%3D17640\&ei=7gXzU66kAoLIyAPj_ICgDA\&usg =AFQjCNHpmABnwjChso22m1kVV0Qq5XvcRQ\&bvm=bv.73231344,d.b GQ (Effective: 19/08/2014).

Cohen, R.I., Rottkamp, D.M., Maric, D., Barker, J.L., and Hudson, L.D. (2003). A role for semaphorins and neuropilins in oligodendrocyte guidance. Journal of neurochemistry $85,1262-1278$.

Collazo, A., Bronner-Fraser, M., and Fraser, S.E. (1993). Vital dye labelling of Xenopus laevis trunk neural crest reveals multipotency and novel pathways of migration. Development 118, 363-376.

Dalby, B., Cates, S., Harris, A., Ohki, E.C., Tilkins, M.L., Price, P.J., and Ciccarone, V.C. (2004). Advanced transfection with Lipofectamine 2000 reagent: primary neurons, siRNA, and high-throughput applications. Methods 33, 95103.

Database, T.G.H.G. (2014a). Complete information for SEMA3D gene (proteincoding), sema domain, immunoglobulin domain (Ig), short basic domain, secreted, (semaphorin) 3D, including: function, proteins, disorders, pathways, orthologs, and expression. GeneCards - The Human Gene Compendium. Crown Human Genome Center, Department of Molecular Genetics, the Weizmann Institute of Science

Database, T.G.H.G. (2014b). SEMA3E, SEMA3E gene, SEM3E protein, SEM3E antibody, Sema Domain, Immunoglobulin Domain (Ig), Short Basic Domain, Secreted, (Semaphorin) 3E, SEMAH, KIAA0331, M-Sema H, M-SEMAH, M-SemaK, coll-5, semaphorin-3E, . Crown Human Genome Center, Department of Molecular Genetics, the Weizmann Institute of Science

De Bellard, M.E., Rao, Y., and Bronner-Fraser, M. (2003). Dual function of Slit2 in repulsion and enhanced migration of trunk, but not vagal, neural crest cells. The Journal of cell biology 162, 269-279.

de Zegher, F., Lagae, L., Declerck, D., and Vinckier, F. (1995). Kallmann syndrome and delayed puberty associated with agenesis of lateral maxillary incisors. Journal of craniofacial genetics and developmental biology 15, 87-89. 
den Dunnen, J.T., and Antonarakis, S.E. (2000). Mutation nomenclature extensions and suggestions to describe complex mutations: a discussion. Human mutation $15,7-12$.

Dode, C., Levilliers, J., Dupont, J.M., De Paepe, A., Le Du, N., Soussi-Yanicostas, N., Coimbra, R.S., Delmaghani, S., Compain-Nouaille, S., Baverel, F., Pecheux, C., Le Tessier, D., Cruaud, C., Delpech, M., Speleman, F., Vermeulen, S., Amalfitano, A., Bachelot, Y., Bouchard, P., Cabrol, S., Carel, J.C., Delemarre-van de Waal, H., Goulet-Salmon, B., Kottler, M.L., Richard, O., Sanchez-Franco, F., Saura, R., Young, J., Petit, C., and Hardelin, J.P. (2003). Loss-of-function mutations in FGFR1 cause autosomal dominant Kallmann syndrome. Nature genetics 33, 463-465.

Dou, Y., Milne, T.A., Ruthenburg, A.J., Lee, S., Lee, J.W., Verdine, G.L., Allis, C.D., and Roeder, R.G. (2006). Regulation of MLL1 H3K4 methyltransferase activity by its core components. Nature structural \& molecular biology 13, 713-719.

Duband, J.L., Rocher, S., Chen, W.T., Yamada, K.M., and Thiery, J.P. (1986). Cell adhesion and migration in the early vertebrate embryo: location and possible role of the putative fibronectin receptor complex. The Journal of cell biology 102, 160-178.

Duband, J.L., and Thiery, J.P. (1982). Distribution of fibronectin in the early phase of avian cephalic neural crest cell migration. Developmental biology 93, 308323.

Egea, J., and Klein, R. (2007). Bidirectional Eph-ephrin signaling during axon guidance. Trends in cell biology 17, 230-238.

Eickholt, B.J., Mackenzie, S.L., Graham, A., Walsh, F.S., and Doherty, P. (1999). Evidence for collapsin-1 functioning in the control of neural crest migration in both trunk and hindbrain regions. Development 126, 2181-2189.

Ernst, P., and Vakoc, C.R. (2012). WRAD: enabler of the SET1-family of H3K4 methyltransferases. Brief Funct Genomics 11, 217-226.

Fields, S., and Song, O. (1989). A novel genetic system to detect protein-protein interactions. Nature 340, 245-246.

Fisher, M.P., and Dingman, C.W. (1971). Role of molecular conformation in determining the electrophoretic properties of polynucleotides in agaroseacrylamide composite gels. Biochemistry 10, 1895-1899.

Flanagan, J.F., Blus, B.J., Kim, D., Clines, K.L., Rastinejad, F., and Khorasanizadeh, S. (2007). Molecular implications of evolutionary differences in CHD double chromodomains. Journal of molecular biology 369, 334-342.

Flaus, A., Martin, D.M., Barton, G.J., and Owen-Hughes, T. (2006). Identification of multiple distinct Snf2 subfamilies with conserved structural motifs. Nucleic acids research 34, 2887-2905.

Gammill, L.S., and Bronner-Fraser, M. (2003). Neural crest specification: migrating into genomics. Nature reviews. Neuroscience 4, 795-805.

GeneTools. (n.d.). Home. http://www.gene-tools.com/ (Effective: 16/07/2014).

Georgiev, G.P. (1972). The structure of transcriptional units in eukaryotic cells. Current topics in developmental biology 7, 1-60.

Gietz, D., St Jean, A., Woods, R.A., and Schiestl, R.H. (1992). Improved method for high efficiency transformation of intact yeast cells. Nucleic acids research 20, 1425.

Gilbert, S.F. (2000). Developmental Biology (6th edn): Sinauer Associates. 
Gitler, A.D., Lu, M.M., and Epstein, J.A. (2004). PlexinD1 and semaphorin signaling are required in endothelial cells for cardiovascular development. Developmental cell 7, 107-116.

Hall, B.D. (1979). Choanal atresia and associated multiple anomalies. The Journal of pediatrics 95, 395-398.

Hall, J.A., and Georgel, P.T. (2007). CHD proteins: a diverse family with strong ties. Biochemistry and cell biology = Biochimie et biologie cellulaire 85, 463-476.

Halloran, M.C., Severance, S.M., Yee, C.S., Gemza, D.L., Raper, J.A., and Kuwada, J.Y. (1999). Analysis of a Zebrafish semaphorin reveals potential functions in vivo. Developmental dynamics : an official publication of the American Association of Anatomists 214, 13-25.

Hanahan, D. (1983). Studies on transformation of Escherichia coli with plasmids. Journal of molecular biology 166, 557-580.

Hanchate, N.K., Giacobini, P., Lhuillier, P., Parkash, J., Espy, C., Fouveaut, C., Leroy, C., Baron, S., Campagne, C., Vanacker, C., Collier, F., Cruaud, C., Meyer, V., Garcia-Pinero, A., Dewailly, D., Cortet-Rudelli, C., Gersak, K., Metz, C., Chabrier, G., Pugeat, M., Young, J., Hardelin, J.P., Prevot, V., and Dode, C. (2012). SEMA3A, a gene involved in axonal pathfinding, is mutated in patients with Kallmann syndrome. PLoS genetics 8, e1002896.

Hardcastle, Z., Chalmers, A.D., and Papalopulu, N. (2000). FGF-8 stimulates neuronal differentiation through FGFR-4a and interferes with mesoderm induction in Xenopus embryos. Current biology : CB 10, 1511-1514.

Hardelin, J.P., Levilliers, J., Blanchard, S., Carel, J.C., Leutenegger, M., PinardBertelletto, J.P., Bouloux, P., and Petit, C. (1993a). Heterogeneity in the mutations responsible for $\mathrm{X}$ chromosome-linked Kallmann syndrome. Human molecular genetics 2, 373-377.

Hardelin, J.P., Levilliers, J., Young, J., Pholsena, M., Legouis, R., Kirk, J., Bouloux, P., Petit, C., and Schaison, G. (1993b). Xp22.3 deletions in isolated familial Kallmann's syndrome. The Journal of clinical endocrinology and metabolism 76, 827-831.

Hardouin, S.N., and Nagy, A. (2000). Mouse models for human disease. Clinical genetics 57, 237-244.

Harland, R.M. (1991). In situ hybridization: an improved whole-mount method for Xenopus embryos. Methods in cell biology 36, 685-695.

Hawker, K., Fuchs, H., Angelis, M.H., and Steel, K.P. (2005). Two new mouse mutants with vestibular defects that map to the highly mutable locus on chromosome 4. International journal of audiology 44, 171-177.

Hedderich, M.C. (2012). Molecular characterization of Ptfla activity during Xenopus embryogenesis. Developmental Biochemistry. Goettingen: Georg-AugustUniversity.

Helling, R.B., Goodman, H.M., and Boyer, H.W. (1974). Analysis of endonuclease R-EcoRI fragments of DNA from lambdoid bacteriophages and other viruses by agarose-gel electrophoresis. Journal of virology 14, 1235-1244.

Herzog, F., Kahraman, A., Boehringer, D., Mak, R., Bracher, A., Walzthoeni, T., Leitner, A., Beck, M., Hartl, F.U., Ban, N., Malmstrom, L., and Aebersold, R. (2012). Structural probing of a protein phosphatase 2A network by chemical cross-linking and mass spectrometry. Science 337, 1348-1352.

HGVS. (2013). Description of sequence changes: examples protein-level http://www.hgvs.org/mutnomen/examplesAA.html\#sub 21/07/2014).

(Effective: 
Hill, J., Donald, K.A., and Griffiths, D.E. (1991). DMSO-enhanced whole cell yeast transformation. Nucleic acids research 19, 5791.

Hittner, H.M., Hirsch, N.J., Kreh, G.M., and Rudolph, A.J. (1979). Colobomatous microphthalmia, heart disease, hearing loss, and mental retardation--a syndrome. Journal of pediatric ophthalmology and strabismus 16, 122-128.

Ho, L., Jothi, R., Ronan, J.L., Cui, K., Zhao, K., and Crabtree, G.R. (2009a). An embryonic stem cell chromatin remodeling complex, esBAF, is an essential component of the core pluripotency transcriptional network. Proceedings of the National Academy of Sciences of the United States of America 106, 5187-5191.

Ho, L., Ronan, J.L., Wu, J., Staahl, B.T., Chen, L., Kuo, A., Lessard, J., Nesvizhskii, A.I., Ranish, J., and Crabtree, G.R. (2009b). An embryonic stem cell chromatin remodeling complex, esBAF, is essential for embryonic stem cell self-renewal and pluripotency. Proceedings of the National Academy of Sciences of the United States of America 106, 5181-5186.

Hrabe de Angelis, M.H., Flaswinkel, H., Fuchs, H., Rathkolb, B., Soewarto, D., Marschall, S., Heffner, S., Pargent, W., Wuensch, K., Jung, M., Reis, A., Richter, T., Alessandrini, F., Jakob, T., Fuchs, E., Kolb, H., Kremmer, E., Schaeble, K., Rollinski, B., Roscher, A., Peters, C., Meitinger, T., Strom, T., Steckler, T., Holsboer, F., Klopstock, T., Gekeler, F., Schindewolf, C., Jung, T., Avraham, K., Behrendt, H., Ring, J., Zimmer, A., Schughart, K., Pfeffer, K., Wolf, E., and Balling, R. (2000). Genome-wide, large-scale production of mutant mice by ENU mutagenesis. Nature genetics 25, 444-447.

Huang da, W., Sherman, B.T., and Lempicki, R.A. (2009a). Bioinformatics enrichment tools: paths toward the comprehensive functional analysis of large gene lists. Nucleic acids research 37, 1-13.

Huang da, W., Sherman, B.T., and Lempicki, R.A. (2009b). Systematic and integrative analysis of large gene lists using DAVID bioinformatics resources. Nature protocols 4, 44-57.

Hubrecht-Laboratorium (Embryologisch Instituut), Nieuwkoop, P.D., and Faber, J. (1967). Normal table of Xenopus laevis (Daudin). A systematical and chronological survey of the development from the fertilized egg till the end of metamorphosis (2. edn). Amsterdam,: North-Holland Pub. Co.

Hurd, E.A., Capers, P.L., Blauwkamp, M.N., Adams, M.E., Raphael, Y., Poucher, H.K., and Martin, D.M. (2007). Loss of Chd7 function in gene-trapped reporter mice is embryonic lethal and associated with severe defects in multiple developing tissues. Mammalian genome : official journal of the International Mammalian Genome Society 18, 94-104.

Illumina. (2011). Using Passive Reference Dyes for Normalization and Troubleshooting in qPCR. http://res.illumina.com/documents/products/technotes/technote_qpcr_using_r ox_normalization.pdf (Effective: 19/08/2014).

Invitrogen. (2010). Platinum®Taq DNA Polymerase http://www.lifetechnologies.com/content/dam/LifeTech/migration/files/pcr/p dfs.par.26652.file.dat/platinumtaq-pps.pdf (Effective: 01/08/2014).

Ito, H., Fukuda, Y., Murata, K., and Kimura, A. (1983). Transformation of intact yeast cells treated with alkali cations. Journal of bacteriology 153, 163-168.

Janssen, N., Bergman, J.E., Swertz, M.A., Tranebjaerg, L., Lodahl, M., Schoots, J., Hofstra, R.M., van Ravenswaaij-Arts, C.M., and Hoefsloot, L.H. (2012). 
Mutation update on the CHD7 gene involved in CHARGE syndrome. Human mutation 33, 1149-1160.

Jin, Z., Chau, M.D., and Bao, Z.Z. (2006). Sema3D, Sema3F, and Sema5A are expressed in overlapping and distinct patterns in chick embryonic heart. Developmental dynamics : an official publication of the American Association of Anatomists 235, 163-169.

Jongmans, M.C., Admiraal, R.J., van der Donk, K.P., Vissers, L.E., Baas, A.F., Kapusta, L., van Hagen, J.M., Donnai, D., de Ravel, T.J., Veltman, J.A., Geurts van Kessel, A., De Vries, B.B., Brunner, H.G., Hoefsloot, L.H., and van Ravenswaaij, C.M. (2006). CHARGE syndrome: the phenotypic spectrum of mutations in the CHD7 gene. Journal of medical genetics 43, 306-314.

Jongmans, M.C., van Ravenswaaij-Arts, C.M., Pitteloud, N., Ogata, T., Sato, N., Claahsen-van der Grinten, H.L., van der Donk, K., Seminara, S., Bergman, J.E., Brunner, H.G., Crowley, W.F., Jr., and Hoefsloot, L.H. (2009). CHD7 mutations in patients initially diagnosed with Kallmann syndrome--the clinical overlap with CHARGE syndrome. Clinical genetics 75, 65-71.

Kallmann, F.J., Schoenfeld, W.A., and Barrera, S.E. (1944). The genetic aspects of primary eunuchoidism. American journal of mental deficiency, 203-236.

Kim, H.G., Bhagavath, B., and Layman, L.C. (2008a). Clinical manifestations of impaired GnRH neuron development and function. Neuro-Signals 16, 165182.

Kim, H.G., Kurth, I., Lan, F., Meliciani, I., Wenzel, W., Eom, S.H., Kang, G.B., Rosenberger, G., Tekin, M., Ozata, M., Bick, D.P., Sherins, R.J., Walker, S.L., Shi, Y., Gusella, J.F., and Layman, L.C. (2008b). Mutations in CHD7, encoding a chromatin-remodeling protein, cause idiopathic hypogonadotropic hypogonadism and Kallmann syndrome. American journal of human genetics $83,511-519$.

Kirby, M.L., and Hutson, M.R. (2010). Factors controlling cardiac neural crest cell migration. Cell adhesion \& migration 4, 609-621.

Kirk, J.M., Grant, D.B., Besser, G.M., Shalet, S., Quinton, R., Smith, C.S., White, M., Edwards, O., and Bouloux, P.M. (1994). Unilateral renal aplasia in Xlinked Kallmann's syndrome. Clinical genetics 46, 260-262.

Kleppe, K., Ohtsuka, E., Kleppe, R., Molineux, I., and Khorana, H.G. (1971). Studies on polynucleotides. XCVI. Repair replications of short synthetic DNA's as catalyzed by DNA polymerases. Journal of molecular biology 56, 341-361.

Knecht, A.K., and Bronner-Fraser, M. (2002). Induction of the neural crest: a multigene process. Nature reviews. Genetics 3, 453-461.

Kontges, G., and Lumsden, A. (1996). Rhombencephalic neural crest segmentation is preserved throughout craniofacial ontogeny. Development 122, 3229-3242.

Korbie, D.J., and Mattick, J.S. (2008). Touchdown PCR for increased specificity and sensitivity in PCR amplification. Nature protocols 3, 1452-1456.

Kosaki, K. (2011). Role of rare cases in deciphering the mechanisms of congenital anomalies: CHARGE syndrome research. Congenital anomalies 51, 12-15.

Krotoski, D.M., Domingo, C., and Bronner-Fraser, M. (1986). Distribution of a putative cell surface receptor for fibronectin and laminin in the avian embryo. The Journal of cell biology 103, 1061-1071. 
Kuriyama, S., and Mayor, R. (2008). Molecular analysis of neural crest migration. Philosophical transactions of the Royal Society of London. Series B, Biological sciences 363, 1349-1362.

Laemmli, U.K. (1970). Cleavage of structural proteins during the assembly of the head of bacteriophage T4. Nature 227, 680-685.

Lalani, S.R., Safiullah, A.M., Fernbach, S.D., Harutyunyan, K.G., Thaller, C., Peterson, L.E., McPherson, J.D., Gibbs, R.A., White, L.D., Hefner, M., Davenport, S.L., Graham, J.M., Bacino, C.A., Glass, N.L., Towbin, J.A., Craigen, W.J., Neish, S.R., Lin, A.E., and Belmont, J.W. (2006). Spectrum of CHD7 mutations in 110 individuals with CHARGE syndrome and genotypephenotype correlation. American journal of human genetics 78, 303-314.

Lalani, S.R., Safiullah, A.M., Molinari, L.M., Fernbach, S.D., Martin, D.M., and Belmont, J.W. (2004). SEMA3E mutation in a patient with CHARGE syndrome. Journal of medical genetics 41 , e94.

Lallier, T.E. (2004). Semaphorin profiling of periodontal fibroblasts and osteoblasts. Journal of dental research 83, 677-682.

Lampe, P.D., and Lau, A.F. (2000). Regulation of gap junctions by phosphorylation of connexins. Archives of biochemistry and biophysics 384, 205-215.

Layman, W.S., Hurd, E.A., and Martin, D.M. (2010). Chromodomain proteins in development: lessons from CHARGE syndrome. Clinical genetics 78, 11-20.

Layman, W.S., McEwen, D.P., Beyer, L.A., Lalani, S.R., Fernbach, S.D., Oh, E., Swaroop, A., Hegg, C.C., Raphael, Y., Martens, J.R., and Martin, D.M. (2009). Defects in neural stem cell proliferation and olfaction in Chd7 deficient mice indicate a mechanism for hyposmia in human CHARGE syndrome. Human molecular genetics 18, 1909-1923.

Lee, P.Y., Costumbrado, J., Hsu, C.Y., and Kim, Y.H. (2012). Agarose gel electrophoresis for the separation of DNA fragments. Journal of visualized experiments : JoVE.

Lepore, J.J., Mericko, P.A., Cheng, L., Lu, M.M., Morrisey, E.E., and Parmacek, M.S. (2006). GATA-6 regulates semaphorin 3C and is required in cardiac neural crest for cardiovascular morphogenesis. The Journal of clinical investigation 116, 929-939.

Li, Y., Bogershausen, N., Alanay, Y., Simsek Kiper, P.O., Plume, N., Keupp, K., Pohl, E., Pawlik, B., Rachwalski, M., Milz, E., Thoenes, M., Albrecht, B., Prott, E.C., Lehmkuhler, M., Demuth, S., Utine, G.E., Boduroglu, K., Frankenbusch, K., Borck, G., Gillessen-Kaesbach, G., Yigit, G., Wieczorek, D., and Wollnik, B. (2011). A mutation screen in patients with Kabuki syndrome. Human genetics 130, 715-724.

Lieblich, J.M., Rogol, A.D., White, B.J., and Rosen, S.W. (1982). Syndrome of anosmia with hypogonadotropic hypogonadism (Kallmann syndrome): clinical and laboratory studies in 23 cases. The American journal of medicine 73, 506-519.

Lofberg, J., Ahlfors, K., and Fallstrom, C. (1980). Neural crest cell migration in relation to extracellular matrix organization in the embryonic axolotl trunk. Developmental biology 75, 148-167.

Luo, Y., Shepherd, I., Li, J., Renzi, M.J., Chang, S., and Raper, J.A. (1995). A family of molecules related to collapsin in the embryonic chick nervous system. Neuron 14, 1131-1140.

Luxan, G., Casanova, J.C., Martinez-Poveda, B., Prados, B., D'Amato, G., MacGrogan, D., Gonzalez-Rajal, A., Dobarro, D., Torroja, C., Martinez, F., 
Izquierdo-Garcia, J.L., Fernandez-Friera, L., Sabater-Molina, M., Kong, Y.Y., Pizarro, G., Ibanez, B., Medrano, C., Garcia-Pavia, P., Gimeno, J.R., Monserrat, L., Jimenez-Borreguero, L.J., and de la Pompa, J.L. (2013). Mutations in the NOTCH pathway regulator MIB1 cause left ventricular noncompaction cardiomyopathy. Nature medicine 19, 193-201.

Marfella, C.G., and Imbalzano, A.N. (2007). The Chd family of chromatin remodelers. Mutation research 618, 30-40.

Martin, D.M., Probst, F.J., Fox, S.E., Schimmenti, L.A., Semina, E.V., Hefner, M.A., Belmont, J.W., and Camper, S.A. (2002). Exclusion of PITX2 mutations as a major cause of CHARGE association. American journal of medical genetics $111,27-30$.

Martin, D.M., Sheldon, S., and Gorski, J.L. (2001). CHARGE association with choanal atresia and inner ear hypoplasia in a child with a de novo chromosome translocation $\mathrm{t}(2 ; 7)(\mathrm{p} 14 ; \mathrm{q} 21.11)$. American journal of medical genetics 99, 115-119.

MGI. (2011). Guidelines for Nomenclature of Genes, Genetic Markers, Alleles, and Mutations in Mouse and Rat http://www.informatics.jax.org/mgihome/nomen/gene.shtml (Effective: 21/07/2014).

Miller, T., Krogan, N.J., Dover, J., Erdjument-Bromage, H., Tempst, P., Johnston, M., Greenblatt, J.F., and Shilatifard, A. (2001). COMPASS: a complex of proteins associated with a trithorax-related SET domain protein. Proceedings of the National Academy of Sciences of the United States of America 98, 12902-12907.

MIPS, GenRE, and GSF. (2008). Navigation: » Corum » Intro http://mips.helmholtzmuenchen.de/genre/proj/corum/complexdetails.html?id=1399 (Effective: 01/08/2014).

Mohrmann, L., and Verrijzer, C.P. (2005). Composition and functional specificity of SWI2/SNF2 class chromatin remodeling complexes. Biochimica et biophysica acta 1681, 59-73.

Molsted, K., Kjaer, I., Giwercman, A., Vesterhauge, S., and Skakkebaek, N.E. (1997). Craniofacial morphology in patients with Kallmann's syndrome with and without cleft lip and palate. The Cleft palate-craniofacial journal : official publication of the American Cleft Palate-Craniofacial Association 34, 417424.

Morin-Kensicki, E.M., and Eisen, J.S. (1997). Sclerotome development and peripheral nervous system segmentation in embryonic zebrafish. Development 124, 159-167.

Mullis, K., Faloona, F., Scharf, S., Saiki, R., Horn, G., and Erlich, H. (1986). Specific enzymatic amplification of DNA in vitro: the polymerase chain reaction. Cold Spring Harbor symposia on quantitative biology 51 Pt 1, 263273.

Newgreen, D., and Thiery, J.P. (1980). Fibronectin in early avian embryos: synthesis and distribution along the migration pathways of neural crest cells. Cell and tissue research 211, 269-291.

Newgreen, D.F., Gibbins, I.L., Sauter, J., Wallenfels, B., and Wutz, R. (1982). Ultrastructural and tissue-culture studies on the role of fibronectin, collagen and glycosaminoglycans in the migration of neural crest cells in the fowl embryo. Cell and tissue research 221, 521-549. 
Ng, S.B., Bigham, A.W., Buckingham, K.J., Hannibal, M.C., McMillin, M.J., Gildersleeve, H.I., Beck, A.E., Tabor, H.K., Cooper, G.M., Mefford, H.C., Lee, C., Turner, E.H., Smith, J.D., Rieder, M.J., Yoshiura, K., Matsumoto, N., Ohta, T., Niikawa, N., Nickerson, D.A., Bamshad, M.J., and Shendure, J. (2010). Exome sequencing identifies MLL2 mutations as a cause of Kabuki syndrome. Nature genetics 42, 790-793.

Nolte, J. (2008). Pluripotency of Spermatogonial stem cell lines. Institute of Human Genetics. Goettingen: Georg-August-University.

OlinkBiosciences. (2010). Doulink II Flourescence User manual. http://www.eurogentec.com/EGT/files/Olink/Duolink-II-Fluorescence-UserManual.pdf (Effective: 14/07/2014).

Opitz, L., Salinas-Riester, G., Grade, M., Jung, K., Jo, P., Emons, G., Ghadimi, B.M., Beissbarth, T., and Gaedcke, J. (2010). Impact of RNA degradation on gene expression profiling. BMC medical genomics 3, 36 .

Osborne, N.J., Begbie, J., Chilton, J.K., Schmidt, H., and Eickholt, B.J. (2005). Semaphorin/neuropilin signaling influences the positioning of migratory neural crest cells within the hindbrain region of the chick. Developmental dynamics : an official publication of the American Association of Anatomists 232, 939-949.

Pagon, R.A., Graham, J.M., Jr., Zonana, J., and Yong, S.L. (1981). Coloboma, congenital heart disease, and choanal atresia with multiple anomalies: CHARGE association. The Journal of pediatrics 99, 223-227.

Parisis, N. (2012). Xenopus laevis as a Model System. Labome.

Peqlab. (n.d.). peqGOLD Pwo-DNA-Polymerase* http://www.peqlab.de/wcms/de/pdf/01-5020_m.pdf (Effective: 31/07/2014).

Perris, R. (1997). The extracellular matrix in neural crest-cell migration. Trends in neurosciences 20, 23-31.

Pingoud, A., Alves, J., and Geiger, R. (1993). Restriction enzymes. Methods Mol Biol 16, 107-200.

Pingoud, A., and Jeltsch, A. (2001). Structure and function of type II restriction endonucleases. Nucleic acids research 29, 3705-3727.

Pinto, G., Abadie, V., Mesnage, R., Blustajn, J., Cabrol, S., Amiel, J., Hertz-Pannier, L., Bertrand, A.M., Lyonnet, S., Rappaport, R., and Netchine, I. (2005). CHARGE syndrome includes hypogonadotropic hypogonadism and abnormal olfactory bulb development. The Journal of clinical endocrinology and metabolism 90, 5621-5626.

Randall, V., McCue, K., Roberts, C., Kyriakopoulou, V., Beddow, S., Barrett, A.N., Vitelli, F., Prescott, K., Shaw-Smith, C., Devriendt, K., Bosman, E., Steffes, G., Steel, K.P., Simrick, S., Basson, M.A., Illingworth, E., and Scambler, P.J. (2009). Great vessel development requires biallelic expression of Chd7 and Tbx 1 in pharyngeal ectoderm in mice. The Journal of clinical investigation 119, 3301-3310.

Rickmann, M., Fawcett, J.W., and Keynes, R.J. (1985). The migration of neural crest cells and the growth of motor axons through the rostral half of the chick somite. Journal of embryology and experimental morphology 90, 437-455.

Ringrose, L., and Paro, R. (2004). Epigenetic regulation of cellular memory by the Polycomb and Trithorax group proteins. Annual review of genetics 38, 413443. 
Rohani, N., Canty, L., Luu, O., Fagotto, F., and Winklbauer, R. (2011). EphrinB/EphB signaling controls embryonic germ layer separation by contact-induced cell detachment. PLoS biology 9, e1000597.

Rovasio, R.A., Delouvee, A., Yamada, K.M., Timpl, R., and Thiery, J.P. (1983). Neural crest cell migration: requirements for exogenous fibronectin and high cell density. The Journal of cell biology 96, 462-473.

Sanger, F., and Coulson, A.R. (1975). A rapid method for determining sequences in DNA by primed synthesis with DNA polymerase. Journal of molecular biology 94, 441-448.

Sanlaville, D., Etchevers, H.C., Gonzales, M., Martinovic, J., Clement-Ziza, M., Delezoide, A.L., Aubry, M.C., Pelet, A., Chemouny, S., Cruaud, C., Audollent, S., Esculpavit, C., Goudefroye, G., Ozilou, C., Fredouille, C., Joye, N., Morichon-Delvallez, N., Dumez, Y., Weissenbach, J., Munnich, A., Amiel, J., Encha-Razavi, F., Lyonnet, S., Vekemans, M., and Attie-Bitach, T. (2006). Phenotypic spectrum of CHARGE syndrome in fetuses with CHD7 truncating mutations correlates with expression during human development. Journal of medical genetics 43, 211-217.

Sanlaville, D., Genevieve, D., Bernardin, C., Amiel, J., Baumann, C., de Blois, M.C., Cormier-Daire, V., Gerard, B., Gerard, M., Le Merrer, M., Parent, P., Prieur, F., Prieur, M., Raoul, O., Toutain, A., Verloes, A., Viot, G., Romana, S., Munnich, A., Lyonnet, S., Vekemans, M., and Turleau, C. (2005). Failure to detect an 8p22-8p23.1 duplication in patients with Kabuki (Niikawa-Kuroki) syndrome. European journal of human genetics : EJHG 13, 690-693.

Sanlaville, D., and Verloes, A. (2007). CHARGE syndrome: an update. European journal of human genetics : EJHG 15, 389-399.

Santen, R.J., and Alvin Paulsen, C. (1972). Hypogonadotropic Eunuchoidism. I. Clinical Study of the Mode of Inheritance. The Journal of Clinical Endocrinology \& Metabolism 36, 1-47.

Sato, M., Tsai, H.J., and Yost, H.J. (2006). Semaphorin3D regulates invasion of cardiac neural crest cells into the primary heart field. Developmental biology 298, 12-21.

Schena, M., Shalon, D., Davis, R.W., and Brown, P.O. (1995). Quantitative monitoring of gene expression patterns with a complementary DNA microarray. Science 270, 467-470.

Scherer, W.F., Syverton, J.T., and Gey, G.O. (1953). Studies on the propagation in vitro of poliomyelitis viruses. IV. Viral multiplication in a stable strain of human malignant epithelial cells (strain HeLa) derived from an epidermoid carcinoma of the cervix. The Journal of experimental medicine 97, 695-710.

Schiestl, R.H., and Gietz, R.D. (1989). High efficiency transformation of intact yeast cells using single stranded nucleic acids as a carrier. Current genetics 16, 339-346.

Schnetz, M.P., Handoko, L., Akhtar-Zaidi, B., Bartels, C.F., Pereira, C.F., Fisher, A.G., Adams, D.J., Flicek, P., Crawford, G.E., Laframboise, T., Tesar, P., Wei, C.L., and Scacheri, P.C. (2010). CHD7 targets active gene enhancer elements to modulate ES cell-specific gene expression. PLoS genetics 6, e1001023.

Schulz, Y., Freese, L., Manz, J., Zoll, B., Volter, C., Brockmann, K., Bogershausen, N., Becker, J., Wollnik, B., and Pauli, S. (2014a). CHARGE and Kabuki syndromes: a phenotypic and molecular link. Human molecular genetics. 
Schulz, Y., Wehner, P., Opitz, L., Salinas-Riester, G., Bongers, E.M., van Ravenswaaij-Arts, C.M., Wincent, J., Schoumans, J., Kohlhase, J., Borchers, A., and Pauli, S. (2014b). CHD7, the gene mutated in CHARGE syndrome, regulates genes involved in neural crest cell guidance. Human genetics.

Schwankhaus, J.D., Currie, J., Jaffe, M.J., Rose, S.R., and Sherins, R.J. (1989). Neurologic findings in men with isolated hypogonadotropic hypogonadism. Neurology 39, 223-226.

Schwarting, G.A., Kostek, C., Ahmad, N., Dibble, C., Pays, L., and Puschel, A.W. (2000). Semaphorin $3 \mathrm{~A}$ is required for guidance of olfactory axons in mice. The Journal of neuroscience : the official journal of the Society for Neuroscience 20, 7691-7697.

Serini, G., Valdembri, D., Zanivan, S., Morterra, G., Burkhardt, C., Caccavari, F., Zammataro, L., Primo, L., Tamagnone, L., Logan, M., Tessier-Lavigne, M., Taniguchi, M., Puschel, A.W., and Bussolino, F. (2003). Class 3 semaphorins control vascular morphogenesis by inhibiting integrin function. Nature 424 , 391-397.

Shapiro, A.L., Vinuela, E., and Maizel, J.V., Jr. (1967). Molecular weight estimation of polypeptide chains by electrophoresis in SDS-polyacrylamide gels. Biochemical and biophysical research communications 28, 815-820.

Sharp, P.A., Sugden, B., and Sambrook, J. (1973). Detection of two restriction endonuclease activities in Haemophilus parainfluenzae using analytical agarose--ethidium bromide electrophoresis. Biochemistry 12, 3055-3063.

Shoval, I., Ludwig, A., and Kalcheim, C. (2007). Antagonistic roles of full-length Ncadherin and its soluble BMP cleavage product in neural crest delamination. Development 134, 491-501.

Siebert, J.R., Graham, J.M., Jr., and MacDonald, C. (1985). Pathologic features of the CHARGE association: support for involvement of the neural crest. Teratology 31, 331-336.

Smith, A., Robinson, V., Patel, K., and Wilkinson, D.G. (1997). The EphA4 and EphB1 receptor tyrosine kinases and ephrin-B2 ligand regulate targeted migration of branchial neural crest cells. Current biology : CB 7, 561-570.

Smith, W.C., and Harland, R.M. (1991). Injected Xwnt-8 RNA acts early in Xenopus embryos to promote formation of a vegetal dorsalizing center. Cell 67, 753765.

SNPdev. (n.d.). dbSNP Short Genetic Variations. http://www.ncbi.nlm.nih.gov/projects/SNP/snp_ref.cgi?rs=147821428 (Effective: 14/08/2014).

Soderlund, D., Canto, P., and Mendez, J.P. (2002). Identification of three novel mutations in the KAL1 gene in patients with Kallmann syndrome. The Journal of clinical endocrinology and metabolism 87, 2589-2592.

Song JJ, K.R. (2008). WDR5 interacts with mixed lineage leukemia (MLL) protein via the histone H3-binding pocket. J Biol Chem.

Spieth, J., and Keller, R.E. (1984). Neural crest cell behavior in white and dark larvae of Ambystoma mexicanum: differences in cell morphology, arrangement, and extracellular matrix as related to migration. The Journal of experimental zoology 229, 91-107.

Steward, M.M., Lee, J.S., O'Donovan, A., Wyatt, M., Bernstein, B.E., and Shilatifard, A. (2006). Molecular regulation of H3K4 trimethylation by ASH2L, a shared subunit of MLL complexes. Nature structural \& molecular biology $13,852-854$. 
Takada, I., Mihara, M., Suzawa, M., Ohtake, F., Kobayashi, S., Igarashi, M., Youn, M.Y., Takeyama, K., Nakamura, T., Mezaki, Y., Takezawa, S., Yogiashi, Y., Kitagawa, H., Yamada, G., Takada, S., Minami, Y., Shibuya, H., Matsumoto, K., and Kato, S. (2007). A histone lysine methyltransferase activated by noncanonical Wnt signalling suppresses PPAR-gamma transactivation. Nature cell biology $9,1273-1285$.

Takeichi, M. (1995). Morphogenetic roles of classic cadherins. Current opinion in cell biology 7, 619-627.

Tellier, A.L., Amiel, J., Delezoide, A.L., Audollent, S., Auge, J., Esnault, D., EnchaRazavi, F., Munnich, A., Lyonnet, S., Vekemans, M., and Attie-Bitach, T. (2000). Expression of the PAX2 gene in human embryos and exclusion in the CHARGE syndrome. American journal of medical genetics 93, 85-88.

Thompson, B.A., Tremblay, V., Lin, G., and Bochar, D.A. (2008). CHD8 is an ATPdependent chromatin remodeling factor that regulates beta-catenin target genes. Molecular and cellular biology 28, 3894-3904.

Thompson BA, T.V., Lin G, Bochar DA. (2008). CHD8 is an ATP-dependent chromatin remodeling factor that regulates beta-catenin target genes. Mol Cell Biol. .

Tosney, K.W. (1978). The early migration of neural crest cells in the trunk region of the avian embryo: an electron microscopic study. Developmental biology 62, 317-333.

Towbin, H., and Gordon, J. (1984). Immunoblotting and dot immunobinding--current status and outlook. Journal of immunological methods 72, 313-340.

Towbin, H., Staehelin, T., and Gordon, J. (1979). Electrophoretic transfer of proteins from polyacrylamide gels to nitrocellulose sheets: procedure and some applications. Proceedings of the National Academy of Sciences of the United States of America 76, 4350-4354.

Towbin, H., Staehelin, T., and Gordon, J. (1992). Electrophoretic transfer of proteins from polyacrylamide gels to nitrocellulose sheets: procedure and some applications. 1979. Biotechnology 24, 145-149.

Trotter, K.W., and Archer, T.K. (2008). The BRG1 transcriptional coregulator. Nuclear receptor signaling 6, e004.

Unified nomenclature for the semaphorins/collapsins. Semaphorin Nomenclature Committee. (1999). Cell 97, 551-552.

Van Meter, T.D., and Weaver, D.D. (1996). Oculo-auriculo-vertebral spectrum and the CHARGE association: clinical evidence for a common pathogenetic mechanism. Clinical dysmorphology 5, 187-196.

Vawter, M.P., Evans, S., Choudary, P., Tomita, H., Meador-Woodruff, J., Molnar, M., Li, J., Lopez, J.F., Myers, R., Cox, D., Watson, S.J., Akil, H., Jones, E.G., and Bunney, W.E. (2004). Gender-specific gene expression in postmortem human brain: localization to sex chromosomes. Neuropsychopharmacology : official publication of the American College of Neuropsychopharmacology 29, 373-384.

Verloes, A. (2005). Updated diagnostic criteria for CHARGE syndrome: a proposal. American journal of medical genetics. Part A 133A, 306-308.

Vissers, L.E., van Ravenswaaij, C.M., Admiraal, R., Hurst, J.A., de Vries, B.B., Janssen, I.M., van der Vliet, W.A., Huys, E.H., de Jong, P.J., Hamel, B.C., Schoenmakers, E.F., Brunner, H.G., Veltman, J.A., and van Kessel, A.G. (2004). Mutations in a new member of the chromodomain gene family cause CHARGE syndrome. Nature genetics 36, 955-957. 
Wain, H.M., Bruford, E.A., Lovering, R.C., Lush, M.J., Wright, M.W., and Povey, S. (2002). Guidelines for human gene nomenclature. Genomics 79, 464-470.

Waterston, R.H., Lindblad-Toh, K., Birney, E., Rogers, J., Abril, J.F., Agarwal, P., Agarwala, R., Ainscough, R., Alexandersson, M., An, P., Antonarakis, S.E., Attwood, J., Baertsch, R., Bailey, J., Barlow, K., Beck, S., Berry, E., Birren, B., Bloom, T., Bork, P., Botcherby, M., Bray, N., Brent, M.R., Brown, D.G., Brown, S.D., Bult, C., Burton, J., Butler, J., Campbell, R.D., Carninci, P., Cawley, S., Chiaromonte, F., Chinwalla, A.T., Church, D.M., Clamp, M., Clee, C., Collins, F.S., Cook, L.L., Copley, R.R., Coulson, A., Couronne, O., Cuff, J., Curwen, V., Cutts, T., Daly, M., David, R., Davies, J., Delehaunty, K.D., Deri, J., Dermitzakis, E.T., Dewey, C., Dickens, N.J., Diekhans, M., Dodge, S., Dubchak, I., Dunn, D.M., Eddy, S.R., Elnitski, L., Emes, R.D., Eswara, P., Eyras, E., Felsenfeld, A., Fewell, G.A., Flicek, P., Foley, K., Frankel, W.N., Fulton, L.A., Fulton, R.S., Furey, T.S., Gage, D., Gibbs, R.A., Glusman, G., Gnerre, S., Goldman, N., Goodstadt, L., Grafham, D., Graves, T.A., Green, E.D., Gregory, S., Guigo, R., Guyer, M., Hardison, R.C., Haussler, D., Hayashizaki, Y., Hillier, L.W., Hinrichs, A., Hlavina, W., Holzer, T., Hsu, F., Hua, A., Hubbard, T., Hunt, A., Jackson, I., Jaffe, D.B., Johnson, L.S., Jones, M., Jones, T.A., Joy, A., Kamal, M., Karlsson, E.K., Karolchik, D., Kasprzyk, A., Kawai, J., Keibler, E., Kells, C., Kent, W.J., Kirby, A., Kolbe, D.L., Korf, I., Kucherlapati, R.S., Kulbokas, E.J., Kulp, D., Landers, T., Leger, J.P., Leonard, S., Letunic, I., Levine, R., Li, J., Li, M., Lloyd, C., Lucas, S., Ma, B., Maglott, D.R., Mardis, E.R., Matthews, L., Mauceli, E., Mayer, J.H., McCarthy, M., McCombie, W.R., McLaren, S., McLay, K., McPherson, J.D., Meldrim, J., Meredith, B., Mesirov, J.P., Miller, W., Miner, T.L., Mongin, E., Montgomery, K.T., Morgan, M., Mott, R., Mullikin, J.C., Muzny, D.M., Nash, W.E., Nelson, J.O., Nhan, M.N., Nicol, R., Ning, Z., Nusbaum, C., O'Connor, M.J., Okazaki, Y., Oliver, K., Overton-Larty, E., Pachter, L., Parra, G., Pepin, K.H., Peterson, J., Pevzner, P., Plumb, R., Pohl, C.S., Poliakov, A., Ponce, T.C., Ponting, C.P., Potter, S., Quail, M., Reymond, A., Roe, B.A., Roskin, K.M., Rubin, E.M., Rust, A.G., Santos, R., Sapojnikov, V., Schultz, B., Schultz, J., Schwartz, M.S., Schwartz, S., Scott, C., Seaman, S., Searle, S., Sharpe, T., Sheridan, A., Shownkeen, R., Sims, S., Singer, J.B., Slater, G., Smit, A., Smith, D.R., Spencer, B., Stabenau, A., Stange-Thomann, N., Sugnet, C., Suyama, M., Tesler, G., Thompson, J., Torrents, D., Trevaskis, E., Tromp, J., Ucla, C., Ureta-Vidal, A., Vinson, J.P., Von Niederhausern, A.C., Wade, C.M., Wall, M., Weber, R.J., Weiss, R.B., Wendl, M.C., West, A.P., Wetterstrand, K., Wheeler, R., Whelan, S., Wierzbowski, J., Willey, D., Williams, S., Wilson, R.K., Winter, E., Worley, K.C., Wyman, D., Yang, S., Yang, S.P., Zdobnov, E.M., Zody, M.C., and Lander, E.S. (2002). Initial sequencing and comparative analysis of the mouse genome. Nature 420, 520-562.

Wegenke, J.D., Uehling, D.T., Wear, J.B., Jr., Gordon, E.S., Bargman, J.G., Deacon, J.S., Herrmann, J.P., and Opitz, J.M. (1975). Familial Kallmann syndrome with unilateral renal aplasia. Clinical genetics 7, 368-381.

Wehner, P. (2012). Analyzing PTK7/RACK1 interaction in neural morphogenesis. Developmental Biochemistry. Goettingen: Georg-August-University.

Wieczerzak, K. (2012). Analysis of coiled coil domain containing 33 protein (CCDC33) and determination of infertility causes in mutant mouse line with 
the deletion of six germ cell-specific genes. Institute of Human Genetics. Goettingen: Georg-August-University.

Williams, M.S. (2005). Speculations on the pathogenesis of CHARGE syndrome. American journal of medical genetics. Part A 133A, 318-325.

Witt, D. (2013). Analyse zur Rolle von pflanzlichen Wirkstoffen und Histondeacetylase-Inhibitoren auf Wachstumsfaktoren und deren Signalwege in Prostatakarzinomzellen. Institute of Human Genetics. Goettingen: GeorgAugust-University.

Woodage, T., Basrai, M.A., Baxevanis, A.D., Hieter, P., and Collins, F.S. (1997). Characterization of the CHD family of proteins. Proceedings of the National Academy of Sciences of the United States of America 94, 11472-11477.

Wright, C.G., Brown, O.E., Meyerhoff, W.L., and Rutledge, J.C. (1986). Auditory and temporal bone abnormalities in CHARGE association. The Annals of otology, rhinology, and laryngology 95, 480-486.

Wysocka, J., Swigut, T., Milne, T.A., Dou, Y., Zhang, X., Burlingame, A.L., Roeder, R.G., Brivanlou, A.H., and Allis, C.D. (2005). WDR5 associates with histone $\mathrm{H} 3$ methylated at $\mathrm{K} 4$ and is essential for $\mathrm{H} 3 \mathrm{~K} 4$ methylation and vertebrate development. Cell 121, 859-872.

Xenbase. (2013). Gene Nomenclature Guidelines http://www.xenbase.org/gene/static/geneNomenclature.jsp (Effective: 21/07/2014).

Xenbase. (n.d.). Introduction to Xenopus, the frog model http://www.xenbase.org/anatomy/intro.do (Effective: 17/07/2014).

Yang, X., Schadt, E.E., Wang, S., Wang, H., Arnold, A.P., Ingram-Drake, L., Drake, T.A., and Lusis, A.J. (2006). Tissue-specific expression and regulation of sexually dimorphic genes in mice. Genome research 16, 995-1004.

Yates, J.A., Menon, T., Thompson, B.A., and Bochar, D.A. (2010). Regulation of HOXA2 gene expression by the ATP-dependent chromatin remodeling enzyme CHD8. FEBS Lett 584, 689-693.

Yazdani, U., and Terman, J.R. (2006). The semaphorins. Genome biology 7, 211.

Yokoyama, A., Wang, Z., Wysocka, J., Sanyal, M., Aufiero, D.J., Kitabayashi, I., Herr, W., and Cleary, M.L. (2004). Leukemia proto-oncoprotein MLL forms a SET1-like histone methyltransferase complex with menin to regulate Hox gene expression. Molecular and cellular biology 24, 5639-5649.

Young, J., Metay, C., Bouligand, J., Tou, B., Francou, B., Maione, L., Tosca, L., Sarfati, J., Brioude, F., Esteva, B., Briand-Suleau, A., Brisset, S., Goossens, M., Tachdjian, G., and Guiochon-Mantel, A. (2012). SEMA3A deletion in a family with Kallmann syndrome validates the role of semaphorin $3 \mathrm{~A}$ in human puberty and olfactory system development. Hum Reprod 27, 14601465.

Yu, H.H., and Kolodkin, A.L. (1999). Semaphorin signaling: a little less per-plexin. Neuron 22, 11-14.

$\mathrm{Yu}$, H.H., and Moens, C.B. (2005). Semaphorin signaling guides cranial neural crest cell migration in zebrafish. Developmental biology 280, 373-385.

Zhang, W., Huang, R.S., Duan, S., and Dolan, M.E. (2009). Gene set enrichment analyses revealed differences in gene expression patterns between males and females. In silico biology 9, 55-63.

Zipper, H., Brunner, H., Bernhagen, J., and Vitzthum, F. (2004). Investigations on DNA intercalation and surface binding by SYBR Green I, its structure 
References

determination and methodological implications. Nucleic acids research 32, e103. 


\section{Appendix}

Table 39: Microarray gene expression analysis

\begin{tabular}{|c|c|c|c|c|c|c|}
\hline \multirow[t]{2}{*}{ No. } & \multirow{2}{*}{$\begin{array}{l}\text { Gene } \\
\text { symbol }\end{array}$} & \multirow[t]{2}{*}{ Gene name } & \multicolumn{3}{|c|}{ Wild-type vs. $C h d 7^{\text {Whi/Whi }}$} & \multirow{2}{*}{$\begin{array}{l}\text { Gene Ontology category and } \\
\text { function in neural crest development* }\end{array}$} \\
\hline & & & $\log 2 \mathrm{FC}$ & P-Value & FDR & \\
\hline \multicolumn{7}{|c|}{ Upregulated genes } \\
\hline 1 & Casp12 & caspase 12 & 1.69 & $3.48 \mathrm{E}-04$ & 4.253 & \\
\hline 2 & Rasl10a & RAS-like, family 10 , member A & 1.65 & $2.56 \mathrm{E}-07$ & 0.086 & \\
\hline 3 & Mt1 & metallothionein 1 & 1.50 & $1.36 \mathrm{E}-04$ & 2.653 & ion homeostasis \\
\hline 4 & Acsbg 1 & $\begin{array}{l}\text { acyl-CoA synthetase bubblegum } \\
\text { family member } 1\end{array}$ & 1.53 & $5.53 \mathrm{E}-06$ & 0.363 & \\
\hline 5 & Pkp1 & plakophilin 1 & 1.41 & 3.83E-04 & 4.513 & Cell adhesion \\
\hline 6 & Foxd3 & forkhead box D3 & 1.30 & $1.51 \mathrm{E}-04$ & 2.768 & $\begin{array}{l}\text { Neural crest cell migration } \\
\text { embryonic organ development } \\
\text { positive regulation of gene expression } \\
\text { chordate embryonic development }\end{array}$ \\
\hline 7 & Vstm21 & $\begin{array}{l}\text { V-set and transmembrane } \\
\text { domain containing 2-like }\end{array}$ & 1.26 & $2.74 \mathrm{E}-04$ & 3.774 & \\
\hline 8 & $\mathrm{Cdh} 22$ & cadherin 22 & 1.21 & $3.06 \mathrm{E}-04$ & 3.896 & Cell adhesion \\
\hline 9 & Sox10 & SRY-box containing gene 10 & 1.16 & $7.26 \mathrm{E}-05$ & 1.952 & $\begin{array}{l}\text { Neural crest cell migration } \\
\text { positive regulation of gene expression } \\
\text { chordate embryonic development }\end{array}$ \\
\hline 10 & Igfbp6 & $\begin{array}{l}\text { insulin-like growth factor } \\
\text { binding protein } 6\end{array}$ & 1.16 & $1.20 \mathrm{E}-04$ & 2.481 & \\
\hline 11 & Pcsk9 & $\begin{array}{l}\text { proprotein } \\
\text { subtilisin/kexin type } 9\end{array}$ & 1.15 & $2.88 \mathrm{E}-04$ & 3.847 & neuron differentiation \\
\hline 12 & $\mathrm{Jph} 4$ & junctophilin 4 & 1.13 & 4.47E-04 & 4.77 & \\
\hline 13 & Mt2 & metallothionein 2 & 1.13 & $2.19 \mathrm{E}-04$ & 3.424 & ion homeostasis \\
\hline 14 & Gfra2 & $\begin{array}{llr}\text { glial cell line } & \text { derived } \\
\text { neurotrophic factor } & \text { family } \\
\text { receptor alpha } 2 & \end{array}$ & 1.13 & $5.25 \mathrm{E}-06$ & 0.363 & $\begin{array}{l}\text { transmembrane receptor protein tyrosine } \\
\text { kinase signaling pathway }\end{array}$ \\
\hline 15 & Grik3 & $\begin{array}{l}\text { glutamate receptor, ionotropic, } \\
\text { kainate } 3\end{array}$ & 1.13 & $1.25 \mathrm{E}-05$ & 0.62 & ion homeostasis \\
\hline 16 & Cdh13 & cadherin 13 & 1.12 & $1.49 \mathrm{E}-04$ & 2.754 & Cell adhesion \\
\hline 17 & Flrt1 & $\begin{array}{l}\text { fibronectin leucine rich } \\
\text { transmembrane protein } 1\end{array}$ & 1.11 & 8.65E05 & 2.097 & \\
\hline 18 & Lmo1 & LIM domain only 1 & 1.06 & 4.25E-04 & 4.642 & \\
\hline 19 & Phf15 & PHD finger protein 15 & 1.06 & $5.28 \mathrm{E}-06$ & 0.363 & \\
\hline 20 & $\begin{array}{l}\text { 2200002K } \\
\text { 05Rik }\end{array}$ & $\begin{array}{l}\text { RIKEN cDNA } 2200002 \mathrm{~K} 05 \\
\text { gene }\end{array}$ & 1.04 & 4.13E-04 & 4.583 & \\
\hline 21 & Fam162b & $\begin{array}{l}\text { family with sequence similarity } \\
162 \text {, member B }\end{array}$ & 1.01 & $3.04 \mathrm{E}-06$ & 0.290 & \\
\hline Dow & egulated g & & & & & \\
\hline 22 & Pcdh19 & protocadherin 19 & -1.00 & $1.06 \mathrm{E}-04$ & 2.271 & Cell adhesion \\
\hline 23 & Vegfc & $\begin{array}{l}\text { vascular endothelial growth } \\
\text { factor C }\end{array}$ & -1.00 & $2.00 \mathrm{E}-06$ & 0.245 & $\begin{array}{l}\text { Neural crest cell guidance } \\
\text { transmembrane receptor protein tyrosine } \\
\text { kinase signaling pathway } \\
\text { vasculature development }\end{array}$ \\
\hline
\end{tabular}


Table 1 (Continued)

\begin{tabular}{|c|c|c|c|c|c|c|}
\hline \multirow[t]{2}{*}{ No. } & \multirow{2}{*}{$\begin{array}{l}\text { Gene } \\
\text { symbol }\end{array}$} & \multirow[t]{2}{*}{ Gene name } & \multicolumn{3}{|c|}{ Wild-type vs. $C h d 7^{W h i / W h i}$} & \multirow{2}{*}{$\begin{array}{l}\text { Gene Ontology category and } \\
\text { function in neural crest development* }\end{array}$} \\
\hline & & & $\log 2 \mathrm{FC}$ & P-Value & FDR & \\
\hline 24 & Tmeff2 & $\begin{array}{l}\text { transmembrane protein with } \\
\text { EGF-like and two follistatin-like } \\
\text { domains } 2\end{array}$ & -1.02 & $1.57 \mathrm{E}-04$ & 2.814 & \\
\hline 25 & $\mathrm{Gm} 2496$ & predicted gene 2496 & -1.02 & $1.04 \mathrm{E}-04$ & 2.262 & \\
\hline 26 & Gpm6a & glycoprotein m6a & -1.03 & $2.75 \mathrm{E}-04$ & 3.774 & \\
\hline 27 & Pdgfc & $\begin{array}{l}\text { platelet-derived growth factor, C } \\
\text { polypeptide }\end{array}$ & -1.04 & $1.55 \mathrm{E}-05$ & 0.68 & $\begin{array}{l}\text { Neural crest cell migration } \\
\text { transmembrane receptor protein tyrosine } \\
\text { kinase signaling pathway }\end{array}$ \\
\hline 28 & Txlnb & taxilin beta & -1.05 & 4.13E-04 & 4.583 & \\
\hline 29 & Sema3c & semaphorin $3 \mathrm{C}$ & -1.05 & $1.46 \mathrm{E}-05$ & 0.666 & $\begin{array}{l}\text { Neural crest cell guidance } \\
\text { vasculature development } \\
\text { cellular component movement }\end{array}$ \\
\hline 30 & $\begin{array}{l}\text { D030007L } \\
\text { 05Rik }\end{array}$ & RIKEN cDNA D030007L05 gene & -1.06 & $2.22 \mathrm{E}-05$ & 0.853 & \\
\hline 31 & Clec1b & $\begin{array}{l}\text { C-type lectin domain family } 1 \text {, } \\
\text { member b }\end{array}$ & -1.06 & $6.23 \mathrm{E}-05$ & 1.752 & \\
\hline 32 & Flrt2 & $\begin{array}{l}\text { fibronectin leucine rich } \\
\text { transmembrane protein } 2\end{array}$ & -1.06 & $7.21 \mathrm{E}-05$ & 1.952 & Neural crest cell migration \\
\hline 33 & Slc $35 \mathrm{~d} 3$ & $\begin{array}{l}\text { solute carrier family } 35 \text {, member } \\
\text { D3 }\end{array}$ & -1.07 & $1.28 \mathrm{E}-04$ & 2.554 & \\
\hline 34 & Clec9a & $\begin{array}{l}\text { C-type lectin domain family } 9 \text {, } \\
\text { member a }\end{array}$ & -1.08 & $2.77 \mathrm{E}-05$ & 1.007 & \\
\hline 35 & Lin7a & lin-7 homolog A (C. elegans) & -1.08 & $5.59 \mathrm{E}-05$ & 1.671 & regulation of neurotransmitter levels \\
\hline 36 & $\begin{array}{l}\text { 6720420G } \\
\text { 18Rik }\end{array}$ & RIKEN cDNA 6720420 G18 gene & -1.08 & $6.03 \mathrm{E}-05$ & 1.744 & \\
\hline 37 & Lhx9 & LIM homeobox protein 9 & -1.09 & $1.39 \mathrm{E}-04$ & 2.676 & \\
\hline 38 & Bdnf & brain derived neurotrophic factor & -1.11 & $2.55 \mathrm{E}-06$ & 0.284 & $\begin{array}{l}\text { Axonogenesis, inner ear development } \\
\text { neuron differentiation }\end{array}$ \\
\hline 39 & $\begin{array}{l}\text { A930038C0 } \\
\text { 7Rik }\end{array}$ & $\begin{array}{l}\text { RIKEN cDNA A930038C07 } \\
\text { gene }\end{array}$ & -1.11 & $1.52 \mathrm{E}-05$ & 0.68 & Extracellular matrix organization \\
\hline 40 & $\mathrm{Gad} 2$ & glutamic acid decarboxylase 2 & -1.17 & $3.21 \mathrm{E}-05$ & 1.21 & regulation of neurotransmitter levels \\
\hline 41 & Saa2 & serum amyloid A 2 & -1.18 & 4.15E-06 & 0.34 & \\
\hline 42 & Pcdh17 & protocadherin 17 & -1.19 & $2.12 \mathrm{E}-04$ & 3.374 & Cell adhesion \\
\hline 43 & St8sia4 & $\begin{array}{l}\text { ST8 alpha-N-acetyl-neuraminide } \\
\text { alpha-2,8-sialyl-transferase } 4\end{array}$ & -1.19 & $8.98 \mathrm{E}-05$ & 2.104 & \\
\hline 44 & $\begin{array}{l}\text { D030024E } \\
\text { 09Rik }\end{array}$ & RIKEN cDNA D030024E09 gene & -1.20 & $6.49 \mathrm{E}-05$ & 1.789 & \\
\hline 45 & Pcdhac2 & $\begin{array}{l}\text { protocadherin alpha subfamily } \mathrm{C} \text {, } \\
2\end{array}$ & -1.24 & $1.85 \mathrm{E}-04$ & 3.108 & Cell adhesion \\
\hline 46 & Tnfaip6 & $\begin{array}{l}\text { tumor necrosis factor alpha } \\
\text { induced protein } 6\end{array}$ & -1.25 & $1.68 \mathrm{E}-04$ & 2.936 & Cell adhesion \\
\hline 47 & Inpp4b & $\begin{array}{l}\text { inositol polyphosphate-4- } \\
\text { phosphatase, type II }\end{array}$ & -1.25 & 4.47E-05 & 1.51 & \\
\hline 48 & Asb4 & $\begin{array}{l}\text { ankyrin repeat and SOCS box- } \\
\text { containing } 4\end{array}$ & -1.26 & $2.60 \mathrm{E}-05$ & 0.96 & \\
\hline 49 & Sema3a & semaphorin $3 \mathrm{~A}$ & -1.26 & $2.21 \mathrm{E}-06$ & 0.26 & $\begin{array}{l}\text { Neural crest cell guidance, Axonogenesis } \\
\text { neuron differentiation } \\
\text { cellular component movement }\end{array}$ \\
\hline $\mathbf{5 0}$ & Hnmt & histamine N-methyltransferase & -1.27 & $1.68 \mathrm{E}-04$ & 2.936 & regulation of neurotransmitter levels \\
\hline 51 & Aldh1a7 & $\begin{array}{l}\text { aldehyde dehydrogenase family } \\
1 \text {, subfamily A7 }\end{array}$ & -1.27 & $1.57 \mathrm{E}-04$ & 2.814 & \\
\hline
\end{tabular}


Table 1 (Continued)

\begin{tabular}{|c|c|c|c|c|c|c|}
\hline \multirow{3}{*}{$\begin{array}{l}\text { No. } \\
52\end{array}$} & \multirow{3}{*}{$\begin{array}{l}\text { Gene } \\
\text { symbol } \\
\text { Zic1 }\end{array}$} & \multirow[t]{2}{*}{ Gene name } & \multicolumn{3}{|c|}{ Wild-type vs. $C h d 7^{\text {Whi/Whi }}$} & \multirow{2}{*}{$\begin{array}{l}\text { Gene Ontology category and } \\
\text { function in neural crest development* }\end{array}$} \\
\hline & & & $\log 2 \mathrm{FC}$ & P-Value & FDR & \\
\hline & & $\begin{array}{l}\text { zinc finger protein of the } \\
\text { cerebellum } 1\end{array}$ & -1.30 & $4.36 \mathrm{E}-04$ & 4.708 & $\begin{array}{l}\text { Neural crest induction } \\
\text { inner ear development } \\
\text { embryonic organ development }\end{array}$ \\
\hline 53 & Dmrta2 & $\begin{array}{l}\text { doublesex and mab-3 related } \\
\text { transcription factor like family } \mathrm{A} 2\end{array}$ & -1.32 & $2.57 \mathrm{E}-04$ & 3.658 & \\
\hline 54 & Pou3f2 & $\begin{array}{l}\text { POU domain, class } 3, \\
\text { transcription factor } 2\end{array}$ & -1.34 & $4.25 \mathrm{E}-04$ & 4.642 & $\begin{array}{l}\text { neuron differentiation } \\
\text { positive regulation of gene expression } \\
\text { cellular component movement } \\
\text { ion homeostasis }\end{array}$ \\
\hline 55 & Slitrk1 & $\begin{array}{l}\text { SLIT and NTRK-like family, } \\
\text { member } 1\end{array}$ & -1.37 & $1.90 \mathrm{E}-07$ & 0.07 & $\begin{array}{l}\text { Neural crest cell guidance, Axonogenesis } \\
\text { neuron differentiation }\end{array}$ \\
\hline 56 & Epha7 & Eph receptor A7 & -1.38 & $5.58 \mathrm{E}-06$ & 0.363 & $\begin{array}{l}\text { Neural crest cell guidance, Axonogenesis, } \\
\text { neuron differentiation, transmembrane } \\
\text { receptor protein tyrosine kinase signaling } \\
\text { pathway, cellular component movement }\end{array}$ \\
\hline 57 & Abca9 & $\begin{array}{l}\text { ATP-binding cassette, sub-family } \\
\text { A, member } 9\end{array}$ & -1.39 & $1.02 \mathrm{E}-06$ & 0.196 & \\
\hline 58 & Gm7325 & predicted gene 7325 & -1.40 & $1.78 \mathrm{E}-04$ & 3.035 & \\
\hline 59 & Zfhx4 & zinc finger homeodomain 4 & -1.42 & $4.81 \mathrm{E}-05$ & 1.553 & \\
\hline 60 & $\begin{array}{l}1700042 \mathrm{O} \\
\text { 10Rik }\end{array}$ & RIKEN cDNA $1700042 \mathrm{O} 10$ gene & -1.43 & $2.62 \mathrm{E}-04$ & 3.668 & \\
\hline 61 & Clvs 1 & clavesin 1 & -1.44 & $1.75 \mathrm{E}-04$ & 3.015 & \\
\hline 62 & Cdh10 & cadherin 10 & -1.44 & $4.50 \mathrm{E}-05$ & 1.51 & Cell adhesion \\
\hline 63 & Col3a1 & collagen, type III, alpha 1 & -1.51 & $1.86 \mathrm{E}-05$ & 0.794 & $\begin{array}{l}\text { Extracellular matrix organization } \\
\text { vasculature development }\end{array}$ \\
\hline 64 & Meox2 & mesenchyme homeobox 2 & -1.51 & $3.15 \mathrm{E}-05$ & 1.114 & $\begin{array}{l}\text { Palate development, limb development } \\
\text { muscle tissue development } \\
\text { chordate embryonic development } \\
\text { vasculature development }\end{array}$ \\
\hline 65 & Mmrn1 & multimerin 1 & -1.52 & 1.01E-04 & 2.229 & \\
\hline 66 & Dach1 & dachshund 1 (Drosophila) & -1.59 & $1.42 \mathrm{E}-06$ & 0.202 & \\
\hline 67 & Anxa1 & annexin A1 & -1.60 & $5.76 \mathrm{E}-06$ & 0.363 & \\
\hline 68 & Tnfsf18 & $\begin{array}{l}\text { tumor necrosis factor (ligand) } \\
\text { superfamily, member } 18\end{array}$ & -1.60 & $1.10 \mathrm{E}-04$ & 2.348 & \\
\hline 69 & Chd7 & $\begin{array}{l}\text { chromodomain helicase DNA } \\
\text { binding protein } 7\end{array}$ & -1.60 & $1.56 \mathrm{E}-07$ & 0.07 & $\begin{array}{l}\text { inner ear development, limb development } \\
\text { embryonic organ development } \\
\text { chordate embryonic development } \\
\text { vasculature development }\end{array}$ \\
\hline 70 & Gm715 & predicted pseudogene 715 & -1.61 & 4.84E-07 & 0.145 & \\
\hline 71 & Myf6 & myogenic factor 6 & -1.64 & $9.57 \mathrm{E}-07$ & 0.196 & $\begin{array}{l}\text { positive regulation of gene expression } \\
\text { muscle tissue development } \\
\text { chordate embryonic development }\end{array}$ \\
\hline 72 & $\operatorname{Scn} 3 \mathrm{a}$ & $\begin{array}{l}\text { sodium channel, voltage-gated, } \\
\text { type III, alpha }\end{array}$ & -1.66 & $5.20 \mathrm{E}-06$ & 0.363 & \\
\hline 73 & Foxp2 & forkhead box P2 & -1.74 & $1.26 \mathrm{E}-04$ & 2.552 & muscle tissue development \\
\hline 74 & Rspo2 & $\begin{array}{l}\text { R-spondin } 2 \text { homolog (Xenopus } \\
\text { laevis) }\end{array}$ & -1.74 & 4.05E-04 & 4.583 & \\
\hline 75 & Olig3 & $\begin{array}{l}\text { oligodendrocyte transcription } \\
\text { factor } 3\end{array}$ & -1.79 & $1.29 \mathrm{E}-05$ & 0.62 & \\
\hline 76 & Armc4 & armadillo repeat containing 4 & -1.85 & $8.26 \mathrm{E}-07$ & 0.196 & \\
\hline 77 & Tnnc2 & troponin $\mathrm{C} 2$, fast & -1.85 & $5.45 \mathrm{E}-06$ & 0.363 & \\
\hline 78 & Sema3d & semaphorin 3D & -1.87 & $1.36 \mathrm{E}-06$ & 0.20 & Neural crest cell guidance \\
\hline 79 & Pcdh10 & protocadherin 10 & -1.96 & $1.33 \mathrm{E}-06$ & 0.20 & Cell adhesion \\
\hline 80 & LOC552873 & hypothetical LOC552873 & -2.01 & $1.22 \mathrm{E}-05$ & 0.62 & \\
\hline
\end{tabular}


Appendix

Table 1 (Continued)

\begin{tabular}{|c|c|c|c|c|c|c|}
\hline \multirow[t]{2}{*}{ No. } & \multirow{2}{*}{$\begin{array}{l}\text { Gene } \\
\text { symbol }\end{array}$} & \multirow[t]{2}{*}{ Gene name } & \multicolumn{3}{|c|}{ Wild-type vs. $C h d 7^{\text {Whi/Whi }}$} & \multirow{2}{*}{$\begin{array}{l}\text { Gene Ontology category and } \\
\text { function in neural crest development* }\end{array}$} \\
\hline & & & $\log 2 \mathrm{FC}$ & P-Value & FDR & \\
\hline 81 & Lrrn3 & $\begin{array}{l}\text { leucine rich repeat protein } 3 \text {, } \\
\text { neuronal }\end{array}$ & -2.07 & $9.13 \mathrm{E}-06$ & 0.50 & \\
\hline 82 & Zic4 & $\begin{array}{l}\text { zinc finger protein of the } \\
\text { cerebellum } 4\end{array}$ & -2.09 & $3.80 \mathrm{E}-04$ & 4.513 & Neural crest induction \\
\hline 83 & Vit & vitrin & -2.14 & $5.04 \mathrm{E}-08$ & 0.036 & Extracellular matrix organization \\
\hline 84 & Alx 1 & ALX homeobox 1 & -2.15 & 7.09E-07 & 0.191 & $\begin{array}{l}\text { Palate development } \\
\text { inner ear development } \\
\text { limb development } \\
\text { embryonic organ development } \\
\text { positive regulation of gene expression } \\
\text { chordate embryonic development }\end{array}$ \\
\hline 85 & Nts & neurotensin & -2.15 & $5.37 \mathrm{E}-08$ & 0.036 & \\
\hline 86 & $\begin{array}{l}\text { 9530003O } \\
\text { 04Rik }\end{array}$ & RIKEN cDNA 9530003004 gene & -2.16 & $1.17 \mathrm{E}-04$ & 2.464 & \\
\hline 87 & Dach2 & dachshund 2 (Drosophila) & -2.16 & $3.74 \mathrm{E}-04$ & 4.469 & \\
\hline 88 & Slitrk6 & $\begin{array}{l}\text { SLIT and NTRK-like family, } \\
\text { member } 6\end{array}$ & -2.23 & $1.53 \mathrm{E}-07$ & 0.07 & $\begin{array}{l}\text { Neural crest cell guidance, Axonogenesis } \\
\text { neuron differentiation }\end{array}$ \\
\hline 89 & Pln & phospholamban & -2.43 & $3.07 \mathrm{E}-04$ & 3.896 & \\
\hline 90 & Epha3 & Eph receptor A3 & -2.42 & 4.47E-05 & 1.51 & $\begin{array}{l}\text { Neural crest cell guidance } \\
\text { transmembrane receptor protein tyrosine } \\
\text { kinase signaling pathway }\end{array}$ \\
\hline 91 & Epha5 & Eph receptor A5 & -2.48 & $1.29 \mathrm{E}-05$ & 0.62 & $\begin{array}{l}\text { Neural crest cell guidance } \\
\text { transmembrane receptor protein tyrosine } \\
\text { kinase signaling pathway }\end{array}$ \\
\hline 92 & Gm5127 & predicted gene 5127 & -2.50 & $1.35 \mathrm{E}-04$ & 2.639 & \\
\hline 93 & Myh3 & $\begin{array}{l}\text { myosin, heavy polypeptide } 3 \text {, } \\
\text { skeletal muscle, embryonic }\end{array}$ & -2.52 & $3.24 \mathrm{E}-04$ & 4.088 & \\
\hline 94 & Chl1 & $\begin{array}{l}\text { cell adhesion molecule with } \\
\text { homology to L1CAM }\end{array}$ & -2.56 & 4.79E-06 & 0.363 & $\begin{array}{l}\text { Cell adhesion, Axonogenesis } \\
\text { neuron differentiation } \\
\text { cellular component movement }\end{array}$ \\
\hline 95 & Tecrl & $\begin{array}{l}\text { trans-2,3-enoyl-CoA reductase- } \\
\text { like }\end{array}$ & -2.61 & $8.55 \mathrm{E}-05$ & 2.097 & \\
\hline 96 & Csn3 & casein kappa & -2.68 & $4.99 \mathrm{E}-10$ & 0.001 & \\
\hline 97 & $\begin{array}{l}\text { D030025E } \\
\text { 07Rik }\end{array}$ & RIKEN cDNA D030025E07 & -2.72 & 4.13E-08 & 0.036 & \\
\hline 98 & Myog & myogenin & -3.50 & $5.52 \mathrm{E}-06$ & 0.363 & $\begin{array}{l}\text { positive regulation of gene expression } \\
\text { muscle tissue development }\end{array}$ \\
\hline
\end{tabular}

Differentially expressed genes with a $\log 2$ fold-change $(\mathrm{FC}) \leq-1$ or $\geq 1$ and a false discovery rate (FDR) $<5 \%$. Genes are listed in the order of the most upregulated to the most downregulated gene. Selected gene ontology categories are shown (GO:0060021: palate development, GO:0007155 cell adhesion, GO:0007409 axonogenesis, GO:0048839 inner ear development, GO:0060173 limb development, GO:0030182 neuron differentiation, GO:0048568 embryonic organ development, GO:0010628 positive regulation of gene expression, GO:0060537 muscle tissue development, GO:0007169 transmembrane receptor protein tyrosine kinase signaling pathway, GO:0001944 vasculature development, GO:0043009 chordate embryonic development, GO:0006928 cellular component movement, GO:0001505 regulation of neurotransmitter levels. GO:0050801 ion homeostasis, GO:0030198 extracellular matrix organization).

* Kirby and Hutson (2010)

Table modified after Schulz et al. (2014b). 


\section{Curriculum vitae}

\section{Curriculum vitae}

\section{Personal}

\begin{tabular}{ll}
\hline Name: & Schulz \\
Surname: & Yvonne \\
Date of birth: & 10/20/1983 \\
Place of birth: & Hamburg, Germany \\
Nationality: & german \\
Address: & Schulweg 10, 37083 Goettingen \\
E-Mail: & yschulz83@ googlemail.com
\end{tabular}

\section{Work experience}

Since $03 / 2011$

\section{Institute of Human Genetics, Goettingen \\ PhD thesis}

- participation of diverse GGNB Short Method Courses:

- Mouse histology \& in situ expression analyses

- Basic statistics for graduate students in the life sciences

○ Chromatin Immunoprecipitation (CHiP)

- participation of GGNB Professional Skills Course:

- Effective scientific communication: journal papers, seminar or conference presentations, and posters

- Seminar on Good Scientific Practice

- Speaking in public

○ Tailor-Made Job Applications: How to Apply for a Job and be Hired

- Poster presentations at conferences

- European Human Genetics Conference 2012, June 23-26, 2012, Nuremberg, Germany

- European Human Genetics Conference 2013, June 8-11, 2013, Paris, France

- $\quad$ supervision of a bachelor student and students in practical trainings

$05 / 2009-10 / 2009$

research assistant in the Johann-Friedrich-Blumenbach-Institute for Zoology and Anthropology, Georg-August-University, Goettingen 


\section{Curriculum vitae}

\section{Study}

10/2005 - 01/2011 Diploma Biology study, Georg-August-University, Goettingen

- Major field of study: Human Genetic, Developmental Biology, Pytomedicine

- Subsidiary subjects during studies were mathematic, chemistry, botanic, zoology, microbiology, biochemistry, and genetic

- Diploma in the Institute of Human Genetics: Functional studies of CHARGE syndrome, Final grade: A

\section{Publications}

CHD7, the gene mutated in CHARGE syndrome, regulates genes involved in neural crest cell guidance. Schulz Y., Wehner P., Opitz L., Salinas-Riester G., Bongers E.M.H.F, van RavenswaaijArts C.M.A., Wincent J., Schoumans J., Kohlhase J., Borchers A., Pauli S.; Human Genetics, 2014

CHARGE and Kabuki syndromes: A phenotypic and molecular link. Schulz Y., Freese L., Mänz J., Zoll B., Völter C., Brockmann K., Bögershausen N., Becker J., Wollnik B., Pauli S.; Human Molecular Genetics, 2014

Identification and characterization of FAM124B as a novel component of a CHD7 and CHD8 containing complex. Batsukh T., Schulz Y., Wolf S., Rabe T.I., Oellerich T., Urlaub H., Schaefer I.M., Pauli S.; PLoS One, 2012

Ccdc33, a predominantly testis-expressed gene, encodes a putative peroxisomal protein. Kaczmarek K., Niedzialkowska E., Studencka M., Schulz Y., Grzmil P.; Cytogenetic Genome Research, 2009

\section{Advanced trainings / Practical trainings}

02/2004-03/2005 AuPair in New Jersey, USA

04/2005 - 08/2005 Practical training at the Nature and Biodiversity Conservation Union, Bremervoerde

03/2008 - 04/2008 6-week practical training in the rainforest station for reafforestation Bilsa, Ecuador 